\title{
Druckverteilung auf Kontaktflächen unter Forstreifen
}

\author{
Dissertation \\ zur Erlangung des Doktorgrades \\ an der Fakultät für Forstwissenschaften und Waldökologie \\ der Georg-August-Universität Göttingen
}

vorgelegt von Andreas Ebel

geboren in Celle

Göttingen, im Januar 2006 
D7

http://resolver.sub.uni-goettingen.de/purl/?webdoc-674
1. Berichterstatter:
Prof. Dr. Heribert Jacke
2. Berichterstatter:
Prof. Dr. Klaus v. Gadow

Tag der mündlichen Prüfung: 17. Februar 2006 


\section{Vorwort}

Die vorliegende Arbeit entstand am Institut für Forstliche Arbeitswissenschaft und Verfahrenstechnologie der Georg-August-Universität Göttingen. Ermöglicht wurde sie durch die finanzielle Förderung des Ministeriums für Schule, Weiterbildung und Forschung (MSWF) und des Ministeriums für Umwelt und Naturschutz, Landwirtschaft und Verbraucherschutz (MUNLV) des Bundeslandes Nordrhein-Westfalen sowie durch das Unternehmen Michelin Reifenwerke KGaA.

Besonderer Dank gilt Herrn Prof. Dr. H. Jacke, der mir die Bearbeitung des Forschungsthemas angeboten und ermöglicht hat. Dank seines steten Interesses am Fortgang der Arbeit und der intensiven Diskussion anfallender Probleme, boten sich viele konstruktive Anregungen zur Umsetzung des Themas. Herrn Prof. Dr. K. v. Gadow, Institut für Waldinventur und Waldwachstum der Georg-August-Universität Göttingen, danke ich für die Übernahme des Korreferats.

Zu danken ist weiterhin allen Mitarbeitern des Instituts für Forstliche Arbeitswissenschaft und Verfahrenstechnologie.

Ausdrücklich verbunden fühle ich mich Herrn W. Streeß und Herrn D. Weber, denen viele Ideen beim Bau des Radlastsimulators und der Walkmaschine sowie der Lösung technischer Probleme zu verdanken sind.

Einen weiteren großen Beitrag zum Gelingen des Projekts erbrachte Herr A. Quentin, dem ich recht herzlich für das Schreiben zahlreicher Programme und für seine Hilfe bei der statistischen Datenauswertung danke.

Ebenso gebührt mein Dank Herrn D. Drewes, der mir während des gesamten Projekts ein wertvoller Ratgeber war und auf dessen Hilfe ich mich uneingeschränkt verlassen konnte.

Bei Frau C. Bobbert und Frau J. Bielefeld möchte ich mich für ihr außergewöhnliches Engagement während der Datenaufnahme bedanken. An dieser Stelle sei Herr M. Härtel erwähnt, der dem Projekt über zwei Jahre als studentische Hilfskraft zur Verfügung stand und dem ich ebenfalls Dank schulde.

Ebenso gebührt mein Dank zahlreichen Mitarbeitern der beteiligten Reifenhersteller, die mir bei Fragen zur Seite standen. Zu nennen sind hier insbesondere: Herr C.-G. Victor und Herr P. Müller (Trelleborg), Herr Ch. Fensel und Herr P. Köhne (Nokian), Herr H.-E. Metz (Michelin) sowie Herr R. Krieger (CGS). Des Weiteren möchte ich mich für die unentgeltliche Bereitstellung der Forstreifen bei allen beteiligten Reifenherstellern bedanken.

Herrn Prof. Dr. D. Matthies und Herrn Dr. M. Ziesak vom Lehrstuhl für Forstliche Arbeitswissenschaft und Angewandte Informatik der Technischen Universität München danke ich für die in allen Phasen des Projekts gegebenen wertvollen Anregungen.

Für das gewissenhafte Korrekturlesen des Manuskripts bedanke ich mich bei Herrn J. Bischoff sehr herzlich. 
In der Liste, derer zu danken ist, nimmt Herr Dr. C. Leßner, seinerzeit Doktorand des Instituts für Forstpolitik, Forstgeschichte und Naturschutz der Georg-August-Universität Göttingen, eine besondere Rolle ein. Für die zahlreich geführten Diskussionen und seine Hilfsbereitschaft möchte ich ihm meinen aufrichtigen Dank aussprechen.

Ganz besonderer Dank gebührt meiner Freundin Tanja, die mit viel Geduld und verständnisvoller Rücksichtnahme sowie Opferung zahlreicher Abende und etlicher Wochenenden zum Erfolg dieser Arbeit außerordentlich viel beigetragen hat.

Zu guter Letzt möchte ich mich bei meinen Eltern bedanken, die mich immer uneingeschränkt unterstützten und denen ich diese Arbeit widme.

Göttingen, im Januar 2006

Andreas Ebel 


\section{Inhaltsverzeichnis}

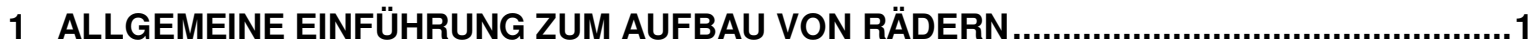

1.1 Rechtliche Grundlagen, RichtLinien Und Normen ................................................

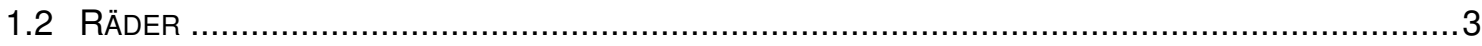

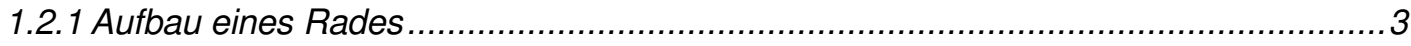

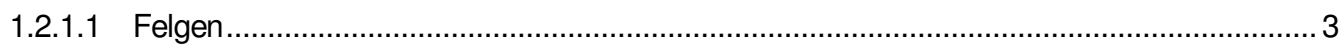

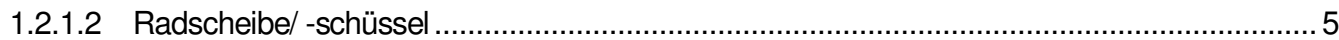

1.2.1.3 Felgenbezeichnung.........................................................................................

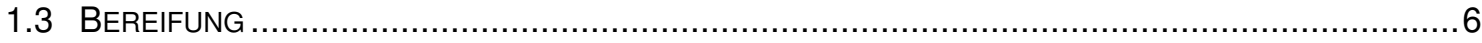

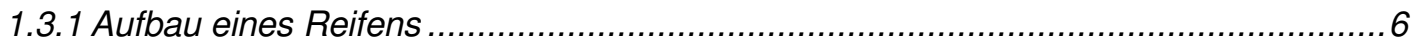

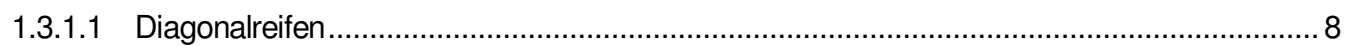

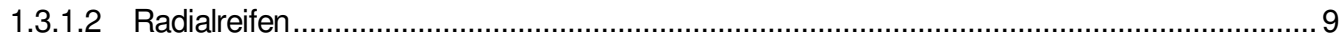

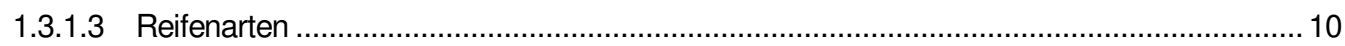

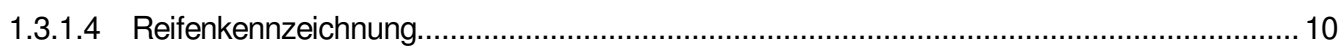

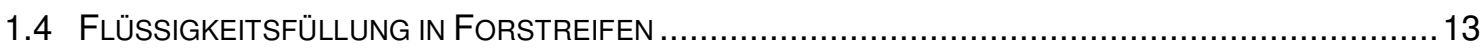

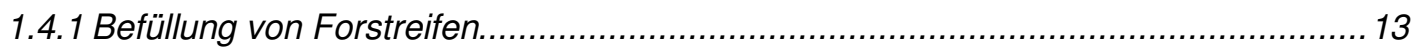

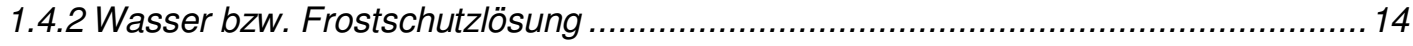

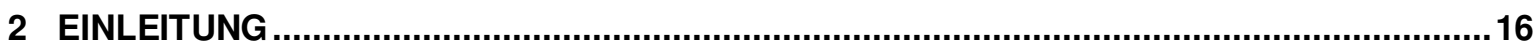

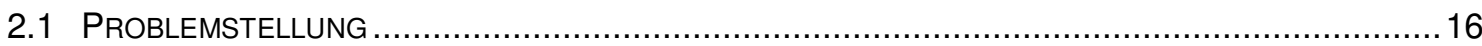

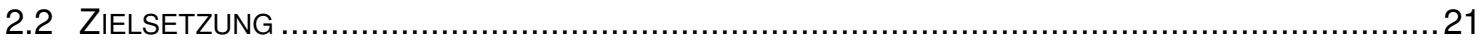

3 MARKTANALYSE DER AUF SELBSTFAHRENDEN FORSTMASCHINEN MONTIERTEN

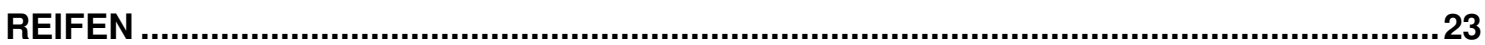

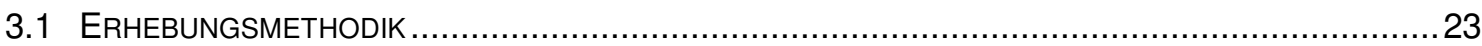

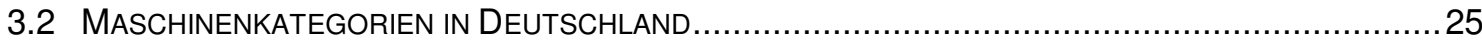

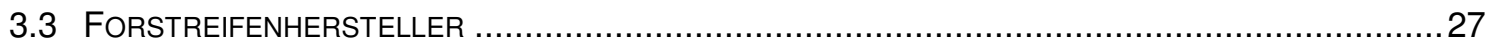

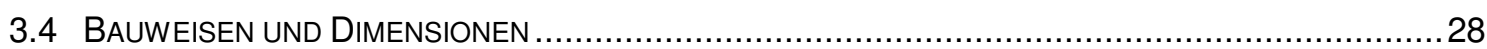

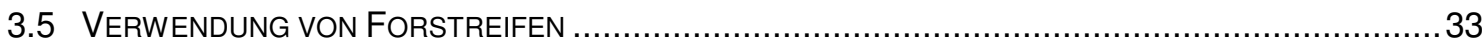

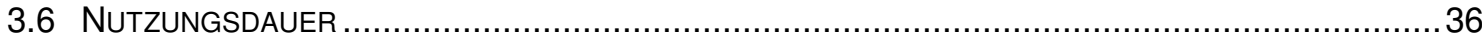

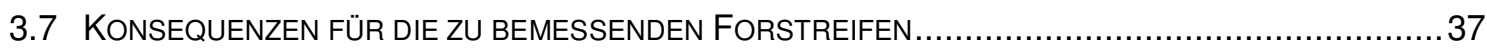

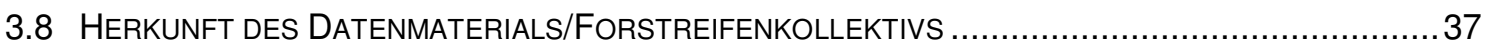

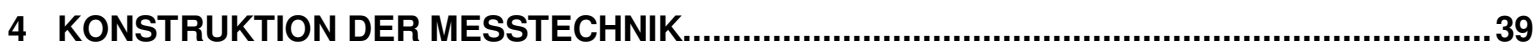

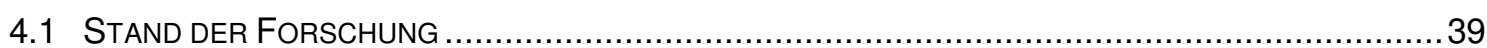

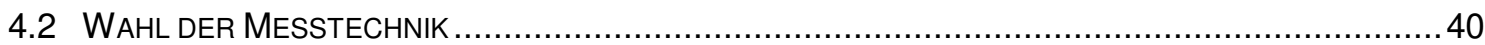

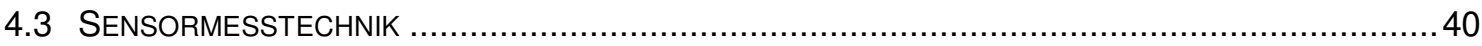


4.3.1 Aufbau und Funktionsweise der Tekscan-Sensormatten...................................... 40

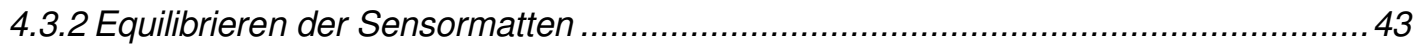

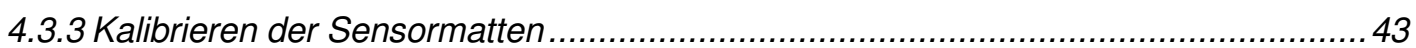

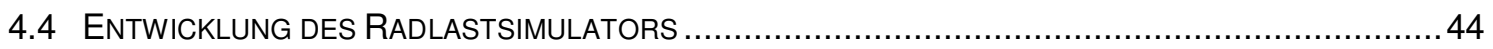

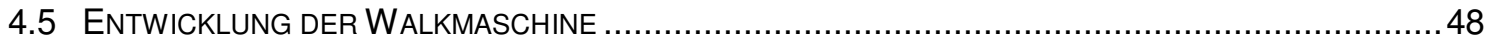

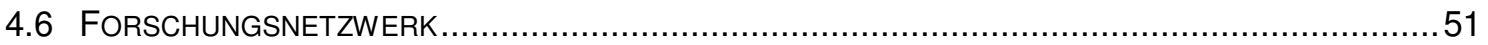

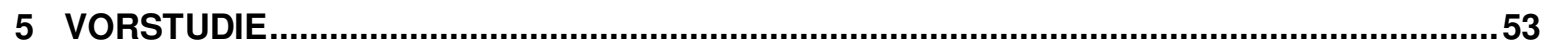

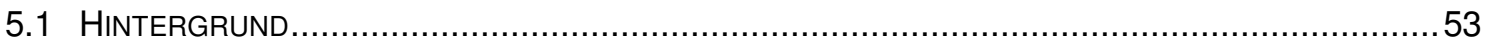

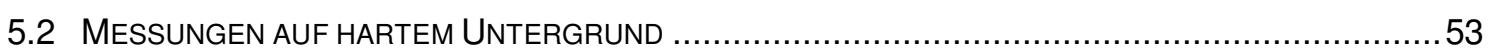

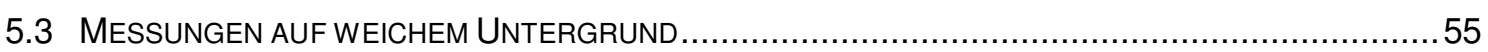

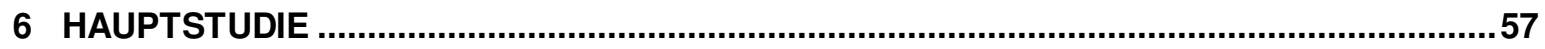

6.1 DATENAUFNAHME

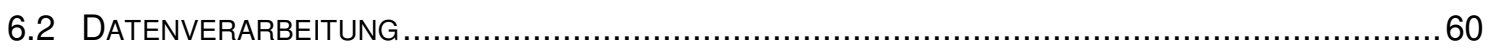

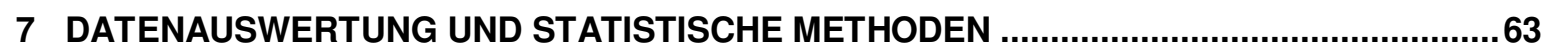

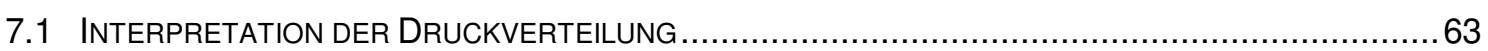

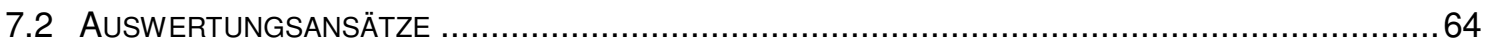

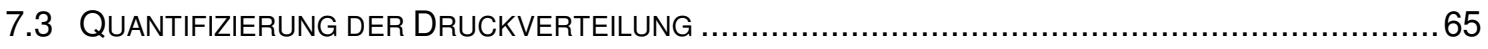

7.4 REgRESSIONSANALYTISCHE ÜBERPRÜFUNG DER DRUCKVERTEILUNG ...................................69

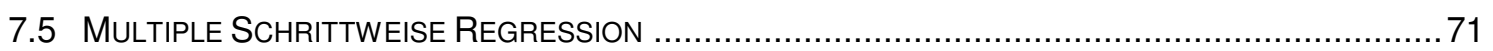

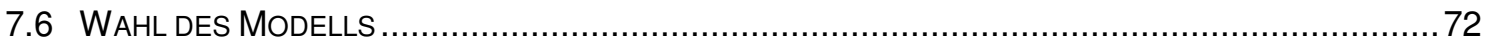

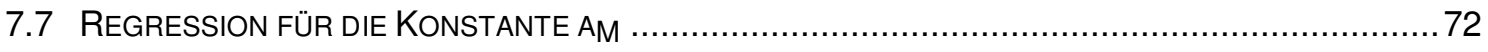

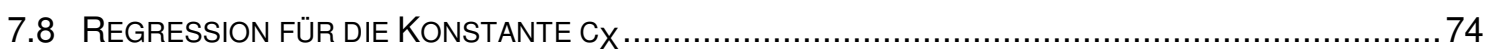

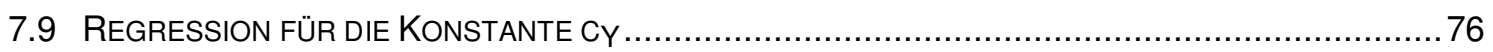

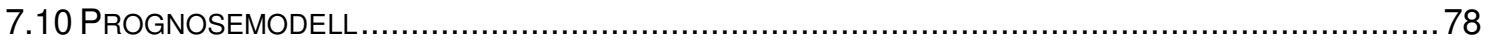

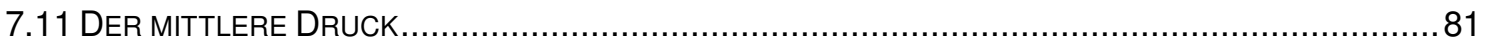

8 DISKUSSION

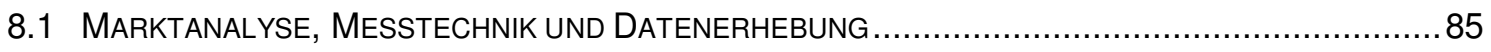

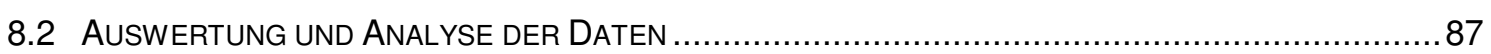

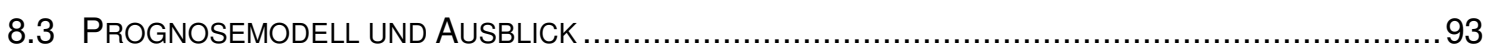

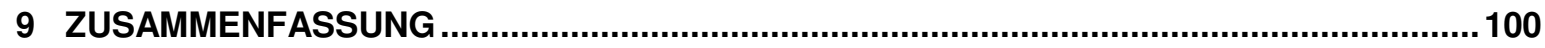

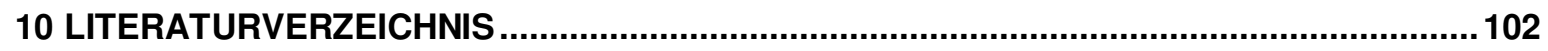

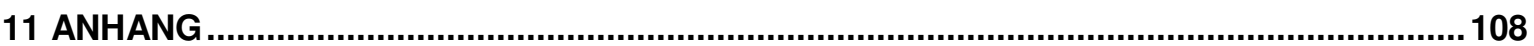




\section{Abbildungsverzeichnis}

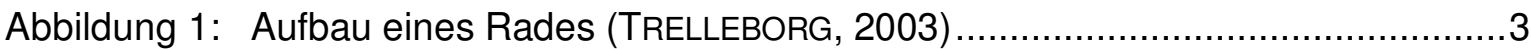

Abbildung 2: Konstruktionsquerschnitt einer Tiefbett- und Steilschulter-Tiefbettfelge

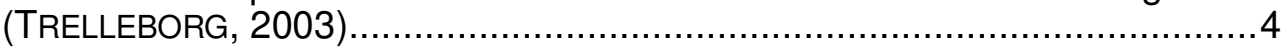

Abbildung 3: Konstruktionsquerschnitt einer Humpfelge (TRELLEBORG, 2003) ................4

Abbildung 4: Schematische Darstellung der Einpresstiefen (TRELLEBORG, 2003) ...........5

Abbildung 5: Aufbau der Radschüssel (Lochbilder und Kennzeichnung, TRELLEBORG, 2003).

Abbildung 6: Schematische Darstellung einer Breitbettfelge mit zweitem Tiefbett (verändert nach BARUM, 2003) ..........................................................6

Abbildung 7: Darstellung eines Radialreifens im Querschnitt (BARUM, 2003) .................6

Abbildung 8: Exemplarische Darstellung eines Traktions- und Softprofils (rechts) anhand von Forstreifen der Firma Trelleborg (Profile 422 und 428) ...........8

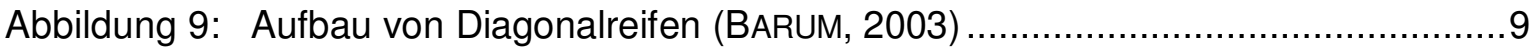

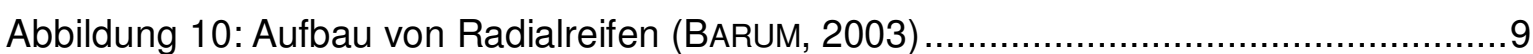

Abbildung 11: Reifenbezeichnung auf der Seitenwand von Forstreifen (verändert nach TRELLEBORG, 2003) .............................................................11

Abbildung 12: Flüssigkeitsbefüllung von Reifen (BARUM, 2003) ..................................13

Abbildung 13: Hanauer Maus und Waterboy (BARUM, 2003) ......................................... 14

Abbildung 14: Hypothetische Kontaktflächendruckverteilung ....................................21

Abbildung 15: Forstmaschinenbestand in Deutschland (Stand: März 2002) ...................26

Abbildung 16: Niederquerschnittreifen in Diagonal- und Radialbauweise, nach Nennbreiten in Millimeter (auf eine Darstellung der 400er und 800er Bereifung wurde in Anbetracht der marginalen Anteile verzichtet).

Abbildung 17: Standardreifen in Diagonal- und Radialbauweise, gegliedert nach Nennbreiten in Zoll

Abbildung 18: Reifenkonglomerat unterschiedlichster Dimensionen von der Firma Trelleborg vor der Göttinger Institutswerkstatt unmittelbar vor Bemessung der Druckverteilung

Abbildung 19: Geometrischer Aufbau des Sensors MATSCAN/3150 (verändert nach TEKSCAN, 2001a) ......................................................................... 41

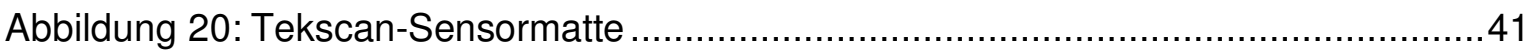

Abbildung 21: Symbolischer Schaltkreis einer Tekscan-Sensormatte (verändert nach TEKSCAN, 2001a).

Abbildung 22: Demonstrationsübersicht des Gebrauchs eines Handles zum Anschluss einer Tekscan-Sensormatte (Tekscan, 2001b)......

Abbildung 23: Bladder

Abbildung 24: Schematische Zeichnung des Radlastsimulators und Beschreibung seiner Bauteile

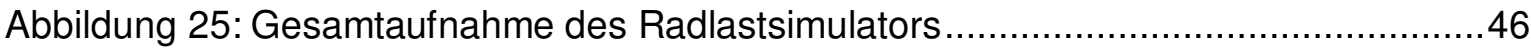

Abbildung 26: Aufnahmerohr und Adapter mit Reifen ................................................ 46

Abbildung 27: Aufnahme- und Schwenkvorrichtung für die Räder ...............................46

Abbildung 28: Hydraulik-Schaltplan des Radlastsimulators (STREEß, 2003)...................47 
Abbildung 29: Messtisch mit eingefassten Sensormatten .......................................... 47

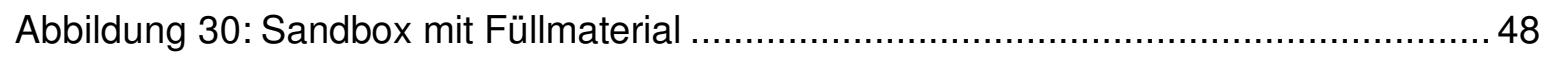

Abbildung 31: Schematische Zeichnung - Aufsicht der Walkmaschine mit Beschreibung der Bauteile...

Abbildung 32: Gesamtaufnahme der Walkmaschine ................................................. 50

Abbildung 33: Elektromotor mit Antriebsachse .................................................... 50

Abbildung 34: Walken des Reifens durch Anpressen ............................................ 51

Abbildung 35: Institutionen, die finanziell, materiell oder durch wissenschaftlichen Austausch das Projekt PrAllCon unterstützten.

Abbildung 36: Kontaktflächendruckverteilung des Nokian Reifens 600/55-26.5

12 PR ELS bei einer Laststufe von $30 \mathrm{kN}$ und einem Reifeninnen-

druck von 2,0 bar, Auszug einer Datenmatrix - Messwerte geben die

Druckverteilung in bar an ................................................................ 54

Abbildung 37: Reifenprofilabdruck mit Füllmaterial Sand der Korngröße $<1 \mathrm{~mm}$............55

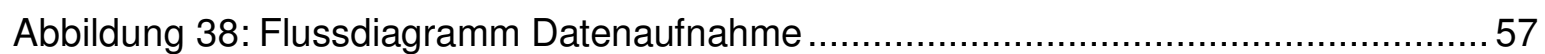

Abbildung 39: Darstellung der Druckbelastung bei konstant gehaltener Radlast von $30 \mathrm{kN}$ und variierenden Fülldrücken (v. I. n. r. 1,0 bar, 3,0 bar, 5,0 bar). Anhand der unterschiedlichen Farbabstufungen (der Druck nimmt ab über die Farben ROT / GELB / GRÜN / BLAU) ist die Verteilung der Druckbelastung zu erkennen.

Abbildung 40: Benutzeroberfläche der I-Scan-Sofware (Tekscan, Version 5.01) ............60

Abbildung 41: Flussdiagramm Datenverarbeitung (alle kursiv geschriebenen Programme sind Eigenentwicklungen des IFA)...

Abbildung 42: Artefakteliminierung am Beispiel einer Kontaktflächendruckverteilung (Radlast 50 kN, Fülldruck 5,0 bar) - der Häufigkeitstabelle am linken Bildrand sind Druckwerte zu entnehmen (links: ohne Ausreißerbeseitigung, rechts: nach Beseitigung von Artefakten)...

Abbildung 43: Dreidimensionale Darstellung einer Kontaktflächendruckverteilung (Reifen T22, Radlast 30 kN, Fülldruck 3,0 bar) .

Abbildung 44: Maximalverteilung des Druckes in Breitenausprägung (Querschnittsdarstellung „Druckrinne“)

Abbildung 45: Verlauf des maximalen Kontaktflächendruckes in Breitenrichtung (Reifen T22, Radlast 30 kN, Fülldruck 3,0 bar)

Abbildung 46: Ausgleich des maximalen Kontaktflächendruckes in Breitenrichtung (Reifen T22, Radlast 30 kN, Fülldruck 3,0 bar) ...

Abbildung 47: Ausgleich des maximalen Kontaktflächendruckes mit der Verschiebung des Koordinatenursprungs ins Funktionsmaximum (Reifen T22, Radlast 30 kN, Fülldruck 3,0 bar).

Abbildung 48: Darstellung der Zusammenhänge bei variierenden Werten der Konstanten a und $\mathrm{c}$.

Abbildung 49: Prognose einer Druckverteilungsfunktion in Breitenrichtung (Radlast 30 kN, Fülldruck 3,0 bar, Nennbreite 378 mm, Durchmesser $1020 \mathrm{~mm})$

Abbildung 50: Prognose einer Druckverteilungsfunktion in Breitenrichtung (Radlast 30 kN, Fülldruck 3,0 bar, Nennbreite 800 mm, Durchmesser $1810 \mathrm{~mm})$ 
Abbildung 51: Prognose einer Druckverteilungsfunktion in Fahrtrichtung (Radlast 30 kN, Fülldruck 3,0 bar, Nennbreite 378 mm, Durchmesser $1020 \mathrm{~mm}$ )

Abbildung 52: Prognose einer Druckverteilungsfunktion in Fahrtrichtung (Radlast 30 kN, Fülldruck 3,0 bar, Nennbreite 800 mm, Durchmesser $1810 \mathrm{~mm}$,)

Abbildung 53: Verlauf des mittleren Kontaktflächendruckes in Breitenrichtung (Reifen T22, Radlast 30 kN, Fülldruck 3,0 bar).

Abbildung 54: Ausgleich des mittleren Kontaktflächendruckes in Breitenrichtung (Reifen T22, Radlast 30 kN, Fülldruck 3,0 bar).

Abbildung 55: Ausgleich des mittleren und maximalen Kontaktflächendruckes in Breitenrichtung (Reifen T22, Radlast 30 kN, Fülldruck 3,0 bar)

Abbildung 56: Relation zwischen $\mathrm{a}_{\mathrm{m}}$ und $\mathrm{a}_{\text {mean }}$ mit eingezeichneter Ausgleichsgeraden.

Abbildung 57: Darstellung des Kontaktflächendruckverlaufs der SkogForsk-Formel und der aus dem Prognosemodell abgeleiteten Werte $a_{m}$ und $a_{\text {mean }}$.

Abbildung 58: Darstellung der Druckwerte der vier Referenzreifen für die verwendeten Formeln 


\section{Tabellenverzeichnis}

Tabelle 1: Mischungsverhältnisse von Frostschutzzusätzen in Abhängigkeit der Temperatur (verändert nach Trelleborg, 2003)

Tabelle 2: Anzahl und Verteilung der in Deutschland arbeitenden Forstmaschinen (Stand: März 2002).

Tabelle 3: Hersteller und Anzahl der in Deutschland eingesetzten Forstreifen (Stand: März 2002)

Tabelle 4: Anzahl und Verteilung der Forstreifen, gegliedert nach Maschinenkategorien (Stand: März 2002)

Tabelle 5: In der forstlichen Praxis auf selbstfahrenden Maschinen eingesetzte Standardreifen (Stand: März 2002)

Tabelle 6: In der forstlichen Praxis auf selbstfahrenden Maschinen eingesetzte Niederquerschnittreifen (Stand: März 2002)

Tabelle 7: Anzahl der Forstreifen auf Harvestern und Forwardern mit unterschiedlichen Fahrwerken (Stand: März 2002).

Tabelle 8: Anzahl der Forstreifen auf Schleppern und Landwirtschaftlichen Schleppern mit Forstausrüstung mit unterschiedlichen Fahrwerken (Stand: März 2002).

Tabelle 9: Verwendung von Niederquerschnittreifen in Diagonalbauweise .................... 34

Tabelle 10: Verwendung von Niederquerschnittreifen in Radialbauweise ....................... 35

Tabelle 11: Verwendung von Standardreifen in Diagonalbauweise .................................. 35

Tabelle 12: Verwendung von Standardreifen in Radialbauweise ..................................... 35

Tabelle 13: Gesamtnutzungsdauer der Maschinen und maximale Nutzungsdauer der darauf montierten Forstreifen

Tabelle 14: Flächenermittlung bei einer Winkeldrehung von $30^{\circ}$ (NOKIAN 600/55-

26.512 PR TRS, Radlast 30 kN, Fülldruck 3,5 bar)................................. 54

Tabelle 15: Variablen des Regressionsmodells ................................................... 70

Tabelle 16: Ergebnisse der multiplen Regressionsanalyse mit der abhängigen Variablen $a_{m}$ 72

Tabelle 17: Regressionsgleichung für die Zielgröße $a_{m}$

Tabelle 18: Ergebnisse der multiplen Regressionsanalyse mit der abhängigen Variablen $\mathrm{c}_{\mathrm{x}}$

Tabelle 19: Regressionsgleichung für die Zielgröße $c_{x}$

Tabelle 20: Ergebnisse der multiplen Regressionsanalyse mit der abhängigen Variablen $\mathrm{c}_{\mathrm{y}}$

Tabelle 21: Regressionsgleichung für die Zielgröße $c_{y}$

Tabelle 22: Auflistung der für einen Vergleich verwendeten 19 Druckermittlungsverfahren nach SAARILAHTI (2002) - ergänzt um die aus dem Prognosemodell abgeleiteten Gleichungen des maximalen $\left(\mathrm{a}_{\mathrm{m}}\right)$ und mittleren Druckes $\left(\mathrm{a}_{\text {mean }}\right)$ 


\section{Abkürzungsverzeichnis}

AG Aktiengesellschaft

ASCII American Standard Code for Information Interchange

B Bestimmtheitsmaß

B.S.R. Bavaria Spezial Rad GmbH

BbodSchG Bundesbodenschutzgesetz

BDI Bundesverband der deutschen Industrie

BNatSchG Bundesnaturschutzgesetz

BS Betriebsstunde

CTIS Central-Tyre-Inflation-System (Reifendruckregelanlage)

DFUV Deutscher Forstunternehmerverband

DIN Deutsches Institut für Normung

DOS Disk Operating System

DOT Department of Transport

E.T.R.T.O. European Tyre and Rim Technical Organization

ECE Economic Commission for Europe

EDV Elektronische Datenverarbeitung

engl. Englisch

EU Europäische Union

EWG Europäische Wirtschaftsgemeinschaft

Fa. Firma

FPA Forsttechnischer Prüfungsausschuss

FSC Forest Stewardship Concil

$\mathrm{GmbH} \quad$ Gesellschaft mit beschränkter Haftung

H/B Verhältnis von Reifenhöhe zu Reifenbreite

HBM Hottinger Baldwin Messtechnik

i. d. R. in der Regel

IFA Institut für Forstliche Arbeitswissenschaft und Verfahrenstechnologie der Universität Göttingen

Inc. Incorporation

ISA Industry Standard Architecture

ISO International Organization for Standardization

KWF Kuratorium für Waldarbeit und Forsttechnik

CEN European Committee for Standardization

LI Load-Index

MAS Maschinenarbeitsstunde

Mio. Million

p. a. pro anno

PEFC Programme for Endorsement of Forest Certification Schemes 
PrAllCon Pressure Allocation on Contact Areas under Forest Tires

PR-Zahl Ply-Rating-Zahl

SEW Süddeutsche Elektromotorenwerke

SI Speed-Index

StVG Straßenverkehrsgesetz

StVZO Straßenverkehrs-Zulassungs-Ordnung

UNO United Nations Organization

upm Umdrehungen pro Minute

vs. versus

vgl. vergleiche

v. I. n. r. von links nach rechts

WdK Wirtschaftsverband der deutschen Kautschukindustrie 


\section{Allgemeine Einführung zum Aufbau von Rädern}

\subsection{Rechtliche Grundlagen, Richtlinien und Normen}

Um einen Einblick über die auf selbstfahrenden Forstmaschinen montierten Räder bzw. den Aufbau von Reifen geben zu können, bedarf es zunächst einer Darstellung der wichtigsten gesetzlichen Grundlagen. Dabei wird sich bei der folgenden Darstellung auf die wesentlichen Aspekte beschränkt.

Der Großteil aller Fahrzeuge wird mit luftgefüllten Gummireifen betrieben. Dieser Umstand unterstreicht die Bedeutung, dass Reifen bestimmte Sicherheits- und Gebrauchsstandards erfüllen müssen. Aus diesem Grund unterliegen u. a. die Reifenabmessungen, die Tragfähigkeiten, die zugelassenen Reifeninnendrücke und Geschwindigkeiten verschiedenen Bestimmungen.

Bezüglich der Gebrauchseigenschaften eines Rades werden bei schweren Fahrzeugen (zu denen auch die Forstmaschinen gehören) vor allem die Fahrsicherheit (Reifensitz auf der Felge sowie Kraftschluss), Widerstandfähigkeit (Geschwindigkeitstüchtigkeit, Platzdruck und Durchstichfestigkeit) sowie Wirtschaftlichkeit (Nutzungsdauer, Abnutzungsbild, Rollwiderstand) beurteilt. Diese Eigenschaften sind bedeutender als z. B. der Fahrkomfort, die Fahrstabilität und das Lenkverhalten, die für den Betrieb auf Krafträdern, Pkw und Nutzfahrzeugen im Vordergrund stehen. Letztere haben allerdings bei der Normung und den rechtlichen Grundlagen mehr Kriterien zu erfüllen.

In Deutschland bildet das StRABENVERKEHRSGESETZ (STVG, 2005) die Rechtsgrundlage für den Bau und den Betrieb von Kraftfahrzeugen. Im § 6 StVG wird der Bundesminister für Verkehr ermächtigt, Rechtsverordnungen und Verwaltungsvorschriften zu erlassen, die Beschaffenheit, Ausrüstung, Prüfung und Kenntlichmachung betreffen. Hierzu zählt auch die STRABENVERKEHRS-ZULASSUNGS-ORDNUNG (STVZO, 2005), in deren § 36 Angaben zu Bereifung und Laufflächen zu finden sind:

(1) Maße und Bauart der Reifen von Fahrzeugen müssen den Betriebsbedingungen, besonders der Belastung und der durch die Bauart bestimmten Höchstgeschwindigkeit des Fahrzeugs, entsprechen ...

(2) Die Räder der Kraftfahrzeuge und Anhänger müssen mit Luftreifen versehen sein, soweit nicht nachstehend andere Bereifungen zugelassen sind. Als Luftreifen gelten Reifen, deren Arbeitsvermögen überwiegend durch den Überdruck des eingeschlossenen Luftinhalts bestimmt wird. Luftreifen an Kraftfahrzeugen und Anhängern müssen am ganzen Umfang und auf der ganzen Breite der Lauffläche mit Profilrillen oder Einschnitten versehen sein.

In den Staaten, die der Europäischen Gemeinschaft angehören, gilt das Subsidiaritätsprinzip. Es besagt, dass in EU-Richtlinien nur der unbedingt notwendige Rahmen vorgegeben wird. Die detaillierte Ausgestaltung hingegen bleibt den einzelnen Mitgliedsländern überlassen. In diesem Zusammenhang ist die Reifenrichtlinie der Europäischen Union 92/23/EWG zu nennen. 
Neben der legislativen Ebene gibt es nationale und internationale Verbände und Organisationen, die einerseits als Interessenvertretung tätig sind, andererseits die innerhalb Europas gebräuchlichen technischen Normen versuchen zu vereinheitlichen.

Der Wirtschaftsverband der deutschen Kautschukindustrie e. V. (WdK, Frankfurt am Main) ist die Interessenvertretung der in Deutschland ansässigen industriellen Unternehmen, die Kautschuk oder kautschukähnliche Kunststoffe verarbeiten (ordentliche Mitglieder) oder als Zulieferer der kautschukverarbeitenden Industrien auftreten (außerordentliche Mitglieder). Wesentliche Ziele des Wdk sind der Informations- und Meinungsaustausch der Mitglieder, das Lösen gemeinschaftlicher Probleme sowie das Erarbeiten eines Branchenstandpunkts als Interessenvertretung. Der WdK nimmt die Interessen der Kautschukindustrie bei Ministerien, Behörden, der EU-Kommission, dem Bundesverband der Deutschen Industrie (BDI) und anderen Organisationen sowie in der Öffentlichkeit wahr. Zudem wird er sowohl im Vorfeld von EUEntscheidungen als auch bei der Umsetzung von EU-Vorschriften in nationales Recht aktiv. Neben der Interessenvertretung ist der WdK Herausgeber unterschiedlicher Leitlinien zu Reifen. So sind vor allem die Leitlinien 094 (Landwirtschaftreifen, Reifensicherheit und Reifenpflege), 104 (Reifen, Montage-Empfehlungen), 105-01 (Reifen und Räder, Begriffe und Bezeichnungssysteme, Reifen) 105-02 (Reifen und Räder, Begriffe und Bezeichnungssysteme, Räder) und die Leitlinien 154 - 162 (Land- und Forstwirtschaftreifen) zu erwähnen.

Das Deutsche Institut für Normung e. V. (DIN, Berlin) hat mit den DIN-Vorschriften 7807, 7808 und 7813 ebenfalls Regelwerke zur Betriebskennung von Reifen herausgegeben. Das DIN versteht sich als ein technisch-wissenschaftlicher Verein, zuständig für die technische Normung in Deutschland sowie für die Vertretung deutscher Interessen in den internationalen und europäischen Normungsorganisationen CEN und ISO.

Eine auf internationaler Ebene tätige Institution ist die der UNO zugehörigen Economic Commission for Europe (ECE) in Genf. Nach der von ihr herausgegebenen ECE-Regelung Nr. 104 sind alle Reifen typisiert und in Verbindung mit der Reifenrichtlinie der Europäischen Union (92/23/EWG) zugelassen. Dabei gilt die ECE-Regelung Nr. 106 für neue Luftreifen, die vor allem - aber nicht nur - auf land- und forstwirtschaftlichen Fahrzeugen sowie landwirtschaftlichen Arbeitsmaschinen eingesetzt werden. In dieser Regelung sind vorwiegend Begriffserläuterung zu Reifenbezeichnungen mit technischen Angaben sowie Informationen zum Genehmigungsverfahren aufgeführt.

Die European Tyre and Rim Technical Organisation (E.T.R.T.O., Brüssel) ist ein freiwilliger Zusammenschluss der Hersteller von Reifen, Felgen und Ventilen. Dieser freiwillige Zusammenschluss zählte im Jahre 2003 einundfünfzig Vollmitglieder aus der Reifenindustrie. Unter anderem sind auch Hersteller von forstlichen Reifen, wie z. B. Nokian Tyres (Ltd., Finnland) und Trelleborg Wheel Systems (Tyre Div. AB, Schweden) in dieser Organisation vertreten. Die E.T.R.T.O. verfolgt u. a. das Ziel, die vielfältigen nationalen Normen innerhalb Europas anzugleichen, um die Austauschbarkeit von Reifen, Felgen und Ventilen zu erleichtern. Dabei ist die Tätigkeit in der E.T.R.T.O. streng auf technische Gesichtspunkte von Reifen, Felgen und Ventilen beschränkt. Weitere Ziele sind die Festlegung von gemeinsamen Abmessungen, Last/Luftdruckzuordnungen und Anwendungsleitlinien sowie die Förderung des ungehinderten Austausches von technischen Informationen. In Anbetracht der Tatsache, dass Reifen einen erheblichen Beitrag zur Verkehrssicherheit leisten, arbeitet die E.T.R.T.O. mit nationalen und internationalen Organisationen und gesetzgebenden Körperschaften 
internationalen Organisationen und gesetzgebenden Körperschaften zusammen, um geeignete Empfehlungen geben zu können. Die E.T.R.T.O. gibt ein Normungshandbuch für „Reifen, Felgen und Ventile - Standards Manual" heraus.

Neben den in Europa richtungsweisenden Normen und Regelungen müssen die Reifenhersteller für den Export von Reifen nach Nordamerika und Kanada, die Richtlinien des Department of Transportation (DOT, Washington D. C., USA) erfüllen.

\subsection{Räder}

\subsubsection{Aufbau eines Rades}

Ein Rad besteht aus der Felge (ringförmiger Teil des Rades, auf der der Reifen montiert wird) und der Radscheibe/-schüssel (Verbindungsteil zwischen Felge und Achsnabe) mit Mittenbohrung und Bolzenlöchern. Die in der forstlichen Praxis montierten Räder, die besonders starken Beanspruchungen/Belastungen ausgesetzt sind, werden darüber hinaus mit individuellen Bauteilen, wie Rundeisen- und Kastenverstärkungen sowie einem Ventilschutz, versehen. Abbildung 1 zeigt die einzelnen Bestandteile eines Rades. Das Rad wird auf dem Flansch der Radnabe, die auf dem Achsschenkelzapfen drehbar gelagert ist, mit Radbefestigungsmuttern oder -schrauben befestigt.

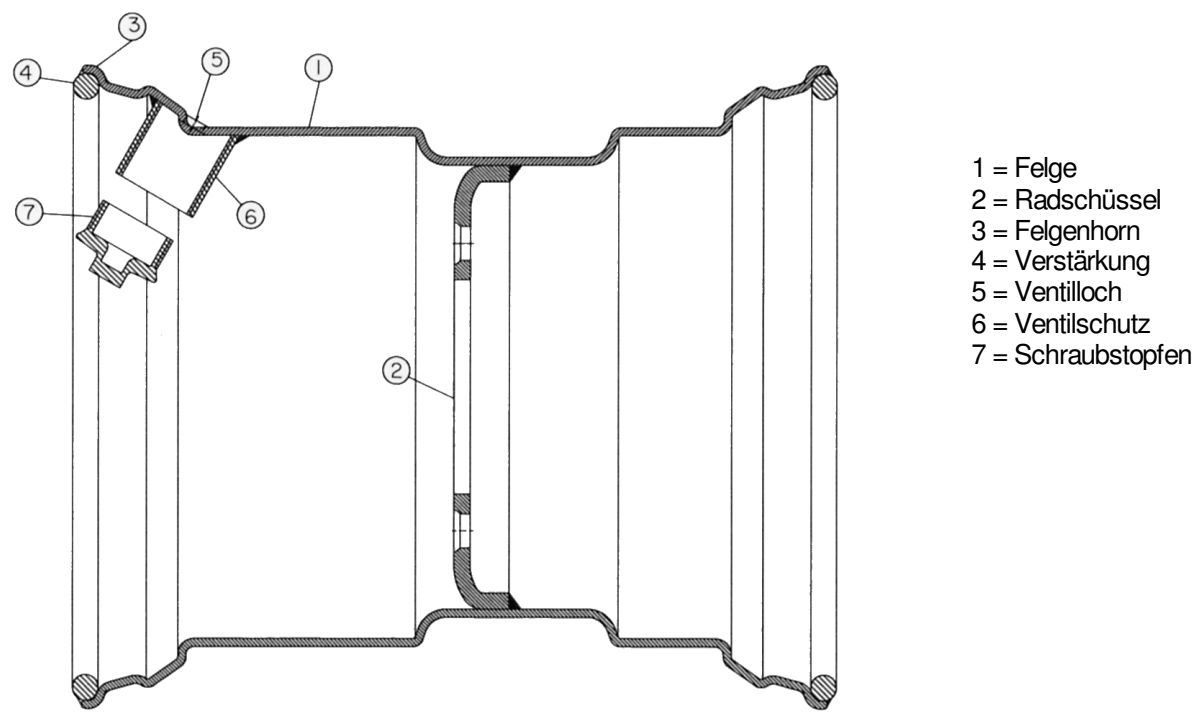

Abbildung 1: Aufbau eines Rades (TRELLEBORG, 2003)

\subsubsection{Felgen}

Grundsätzlich werden in der Forstwirtschaft zwei Felgentypen unterschieden (Abb. 2). Zum einen ist es die Tiefbettfelge (Felgenschulterneigung von $5^{\circ}$ ), die ein hohes Felgenhorn (seitliche Abstützung für den Felgenwulst) aufweist und zum anderen die Steilschulter-Tiefbettfelge (Felgenschulterneigung von $15^{\circ}$ ), die hingegen durch ein flaches Felgenhorn gekennzeichnet ist. Der Felgenquerschnitt kann symmetrisch oder unsymmetrisch sein. 


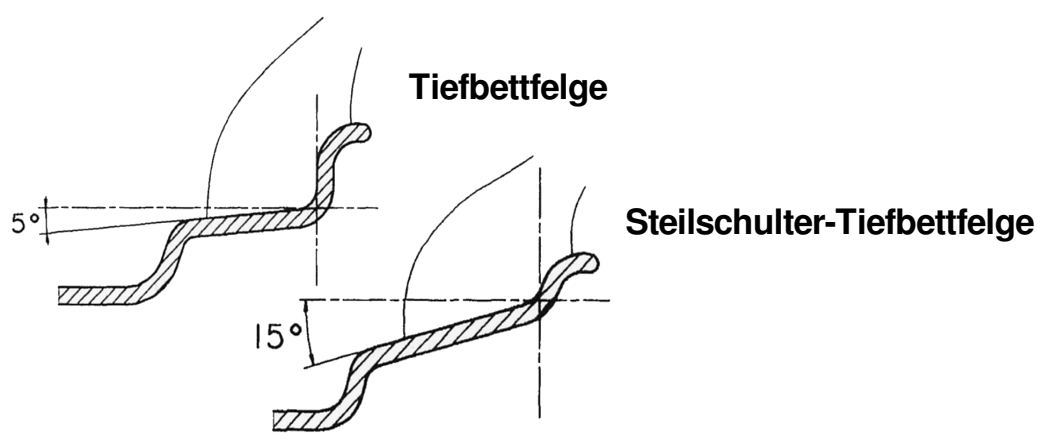

Abbildung 2: Konstruktionsquerschnitt einer Tiefbett- und Steilschulter-Tiefbettfelge (TRELLEBORG, 2003)

Soll das Forstrad mit schlauchlosen (tubeless) Reifen ${ }^{1}$ ausgerüstet werden, so verwendet man Felgen (Tiefbett- oder Steilschulter-Tiefbettfelgen), die auf der Felgenschulter (s. Nr. 2 in Abb. 3), nahe des Tiefbetts, eine rundumlaufende gewalzte Erhöhung (Hump, s. Nr. 1 in Abb. 3) besitzen. Diese sogenannten „Hump-Felgen“ verhindern, dass der Reifenwulst bei starken Seitenkräften von der Felgenschulter ins Tiefbett gedrückt wird, wodurch beim schlauchlosen Reifen schlagartig Luft entweicht. Gleichzeitig sorgen sie für einen festen Sitz des Reifens auf der Felge. Die Steilschulter-Tiefbettfelgen haben zudem den Vorteil, dass infolge der Neigung der Felgenschulter der Reifenwulst beim Befüllen des Reifens mit Luft besonders fest auf die Schulter gepresst wird, womit eine sichere Abdichtung gewährleistet und ein Scheuern der Reifenwülste am Felgenhorn (s. Nr. 3 in Abb. 3) verhindert wird.

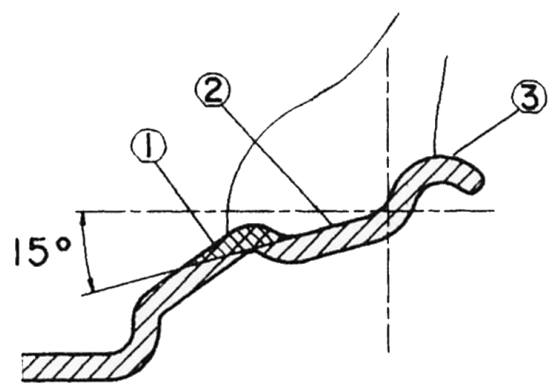

$1=$ Hump

2 = Felgenschulter

3 = Felgenhorn

Abbildung 3: Konstruktionsquerschnitt einer Humpfelge (TRELLEBORG, 2003)

In Abhängigkeit von der Maschinenverwendung - bzw. nach jeweiligem Kundenwunsch - wird die Einpresstiefe (Abstand der Nabenflanschfläche von der Felgenmitte) für die Konstruktion des Rades bestimmt. Je nach Radkonstruktion kann die Einpresstiefe positiv (Nabenflanschfläche der Radschüssel liegt außerhalb der Mittellinie), negativ (Nabenflanschfläche der Radschüssel liegt innerhalb der Mittellinie) oder gleich Null (Nabenflanschfläche der Radschüssel stimmt mit der Mittellinie überein) sein. Bei einer Veränderung der Einpresstiefe ergibt ein höherer positiver Wert eine geringere Spurweite und ein höherer negativer Wert eine größere Spurweite (Abb. 4).

Schlauchlose Reifen unterscheiden sich von Reifen mit Schlauch dadurch, dass sie im Reifeninnern mit einer abdichtenden Gummischicht überzogen sind. Diese Schicht reicht um den Wulst herum bis zur Höhe des Felgenhorns. 


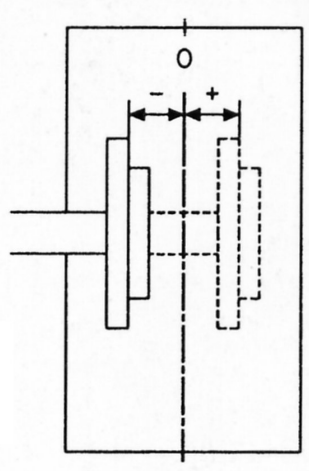

Abbildung 4: Schematische Darstellung der Einpresstiefen (TRELLEBORG, 2003)

\subsubsection{Radscheibe/-schüssel}

Die Radscheibe stellt, wie oben erwähnt, die Verbindung zwischen Felge und Achsnabe her (Abb. 5). Zur Befestigung eines Rades an der Radnabe muss die Radschüssel ein Lochbild aufweisen, das an die Bolzen und Konfiguration der Radnabe angepasst ist. Dabei ist es bei der Fixierung des Rades auf der Radnabe wichtig, möglichst enge Toleranzen beim Mittenlochdurchmesser zu wählen, um eine gute Zentrierung des Rades gewährleisten zu können. Der Lochkreisdurchmesser hat stets enge Toleranzen, ebenso wie die Lochteilung (Abstand zwischen den Befestigungslöchern). Die konisch ausgeführten Radbefestigungsbolzen bzw. Unterlegscheiben sorgen für einen festen Halt des Rades. Gängige Anschlussmaße für Forsträder sind im Anhang 1 in der Tabelle 1.1 angeführt.

A $=$ Mittelochdurchmesser

$\mathrm{B}=$ Lochkreisdurchmesser

C = Befestigungs-/Bolzenloch

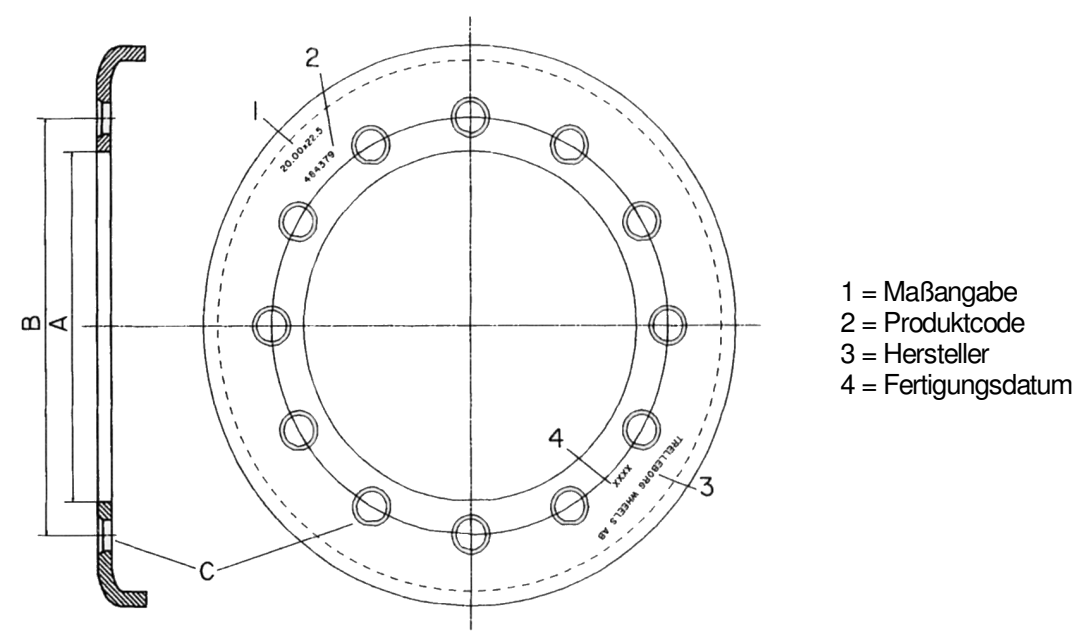

Abbildung 5: Aufbau der Radschüssel (Lochbild und Kennzeichnung, TRELLEBORG, 2003)

\subsubsection{Felgenbezeichnung}

Obwohl die im Forstbereich gefertigten Felgen oft Einzelexemplare und auf die jeweilige Maschine zugeschnitten sind, unterliegen die Felgenabmessungen und -bezeichnungen Normkriterien (z. B. E.T.R.T.O.-Standard Manual, DIN 7827). So wird u. a. an jedem Rad die Felgenbezeichnung vom Hersteller eingeschlagen. Sie besteht grundsätzlich aus zwei Maßen: zum einen aus der Maulweite (Innenabstand zwischen den beiden Felgenhörnern in Zoll, auch Nennweite genannt) und zum anderem aus dem Felgendurchmesser ebenfalls in Zoll (ein 
Zoll = 2,54 cm). Beide Maße werden bei Tiefbettfelgen durch ein „,x“, bei mehrteiligen Felgen durch „,“" getrennt. Eine häufig auf Hinterachsen von Harvestern verwendete Felge ist z. B. 20.00x34 (Abb. 6). Zusätzliche Buchstabenbezeichnungen im Zusammenhang mit der Felgenmaulweite sind Codes für die Felgenquerschnittsform, wie z. B. W oder DW $20.00 \times 34$ (die Bezeichnung W steht für „wide“, engl. für breit und DW steht für „double well“= engl. für Doppel-Tiefbett). Die Felgentypen können durch die Angabe des Felgendurchmessers identifiziert werden: Bei Felgen mit einer Schulterneigung von $15^{\circ}$ endet der Felgendurchmesser auf 0.5 (z. B. 17.5“, 22.5“, 30.5“ ...). Die übrigen Durchmesserangaben beziehen sich auf Felgen mit Schulterneigung von $5^{\circ}$ (z. B. 24“, 34“, 38“ '..).

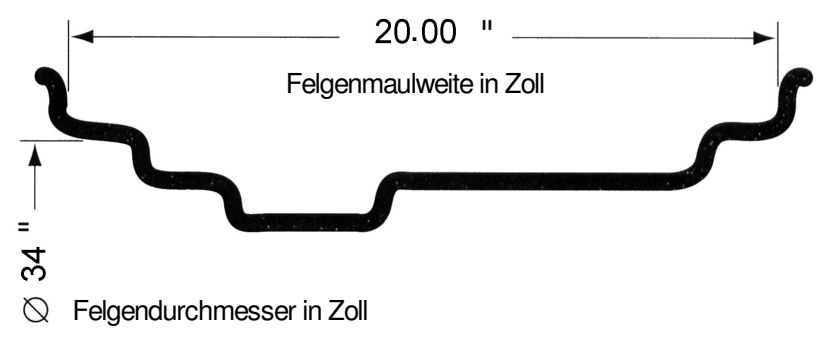

Abbildung 6: Schematische Darstellung einer Breitbettfelge mit zweitem Tiefbett (verändert nach BARUM, 2003)

\subsection{Bereifung}

\subsubsection{Aufbau eines Reifens}

Ein Reifen besteht aus vier Komponenten: Im Einzelnen sind es der Gewebeunterbau, auch Karkasse genannt, die profilierte Lauffläche (Oberbau), die Seitenwand und der Wulst mit den eingelegten Stahldrahtkernen (Abb. 7).

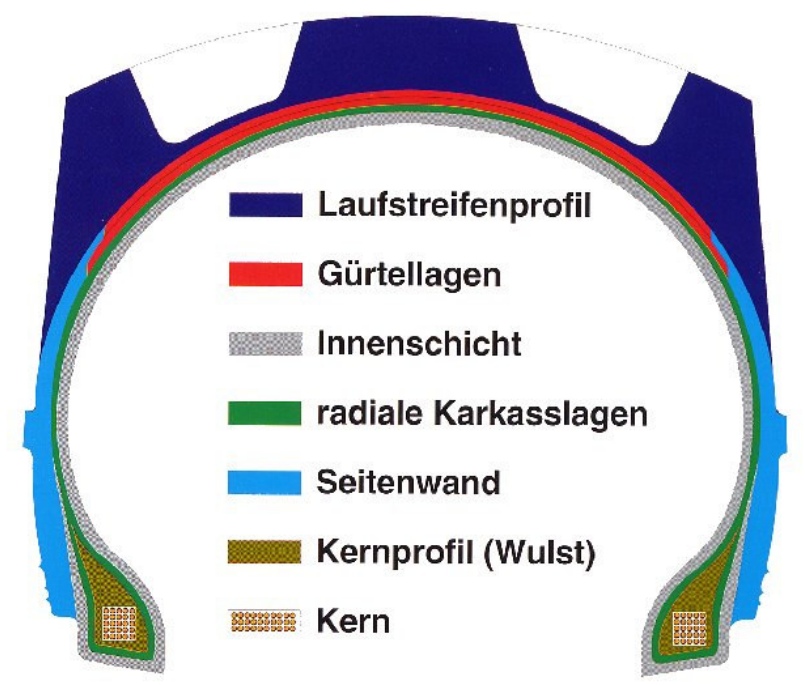

Abbildung 7: Darstellung eines Radialreifens im Querschnitt (BARUM, 2003) 
Die Karkasse ist aus Corden aufgebaut, die aus Kunstseide, Nylon, Rayon oder Polyester bestehen, zum Teil sind die Kunstfaserfäden noch mit Stahlfäden umwickelt (BACHFISCH UND HEINZ, 2000). Die Corde oder „Cordfäden“werden in Lagen übereinander gelegt und verlaufen entweder diagonal (im spitzen Winkel zur Fahrtrichtung) oder radial (im rechten Winkel zur Fahrtrichtung). Zwischen den Corden bestehen keine Berührungspunkte. Von oben und unten werden auf die Gewebelagen dünne Gummiplatten gepresst (kalandriert), die einzelnen Cordfäden fixiert und somit ein Scheuern verhindert. Dieser Unterbau trägt im luftbefüllten Reifen die Last und bestimmt seine Festigkeit.

Das Profil besteht aus Vollgummi und bildet die Lauffläche des Reifens. Die Profilrippen bzw. -stollen (Positivprofil) sind schräg zur Reifenmitte (Querrillenprofil) angeordnet. Damit werden einerseits die Antriebskräfte verlustarm übertragen und andererseits eine optimale Selbstreinigung des Reifens sichergestellt. Die Anzahl der Stollenpaare und das Profildesign variiert mit dem Reifentyp und der Reifengröße.

In der Forstwirtschaft gibt es eine Vielzahl von verwendeten Forstreifenprofilen (aktuell $~ 50$ Profile), die sich obgleich ihrer Variationsfülle generell in zwei Profilkategorien (Traktions- und Softprofil) gliedern lassen (Abb. 8).

Reifen mit Traktionsprofilen werden vorwiegend als Antriebsreifen oder bei einem langfristigen Einsatz der Forstmaschine unter schwierigsten Verhältnissen montiert. Sie zeichnen sich durch eine hohe Zugkraft, robuste Stollen, gute Selbstreinigungseigenschaften, verstärkte Reifenschultern, strapazierfähige Spezialgummimischungen und einem wirkungsvollen Stichschutz aus. In der Regel besitzen sie Profiltiefen von $45 \mathrm{~mm}$ bis $55 \mathrm{~mm}$.

Unter diese Profilkategorie fallen z. B. die Profile TRS L-2, TRS LS-2, TR Forest, FS DT, AS (Nokian), 410, 414, 416, 425, 422 (Trelleborg), FOREXBIB, XM27 (Michelin), Forestry Spezial (Firestone) und TL 1 (Barum).

Softprofile finden vorwiegend auf Maschinen für den Lastentransport und in weniger schwierigen Geländen Verwendung. Insgesamt hat ein Reifen mit Softprofil eine rundere bzw. schrägere Schulter und einen hohen Anteil an Positivprofil bzw. einen geringeren Anteil an Stollenzwischenräumen (Negativprofil). Die höhere Anzahl an Profilblöcken in der Lauffläche gewährleistet einen vibrationsfreien Lauf bei Transporten auf Straßen und Wegen. Neben verstärkten Reifenschultern besitzen auch diese Reifen einen wirkungsvollen Stahlschutz gegen Stiche. Ihre Profiltiefen bewegen sich zwischen $30 \mathrm{~mm}$ und $44 \mathrm{~mm}$.

Den Softprofilen können z. B. die von Nokian entwickelten Profilvarianten LS, ELS und ELS L2 sowie die Trelleborg-Profile 421, 423, 424, 428 zugeordnet werden.

Sowohl auf Traktions- als auch auf Reifen mit Softprofilen ist der Gebrauch von Ketten und Bändern möglich. 


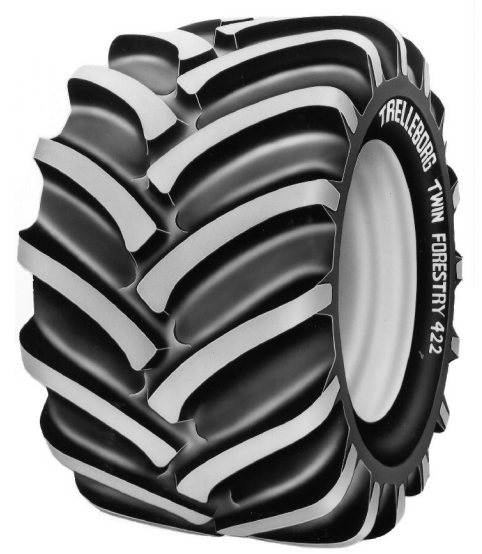

TRELLEBORG TWIN FORESTRY 422

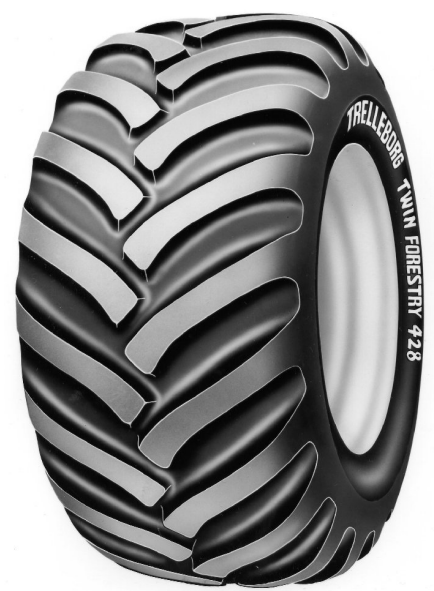

TRELLEBORG TWIN FORESTRY 428

Abbildung 8: Exemplarische Darstellung eines Traktions- und Softprofils (rechts) anhand von Forstreifen der Firma Trelleborg (Profile 422 und 428)

Der Wulst hat die Aufgabe, den Reifen fest auf der Felge zu halten. Um dieses sicherzustellen, besteht der Wulstkern aus gummierten, gewickelten Drahtsträngen. Gegen Beschädigungen bei der Montage schützt den Kernbereich zudem ein zusätzliches Schutzband. Die richtige Abstimmung des Wulstdurchmessers auf den Felgendurchmesser sorgt für den guten Sitz des Reifens, Luftdichtigkeit und Sicherheit gegen Drehen auf der Felge.

Die Seitenwand bzw. Reifenflanke besteht ebenfalls aus Vollgummi. Sie schützt die Karkasse vor mechanischer Beschädigung und trägt die Reifenkennzeichnung.

\subsubsection{Diagonalreifen}

Bei den Diagonalreifen werden die Cordlagen so übereinander gelegt, dass die Karkassfäden mit der Fahrtrichtung des Reifens jeweils einen spitzen Winkel (Fadenwinkel) von 30 - 40 bilden (Abb. 9). Je größer dabei der Fadenwinkel ist, desto weicher wird die Einfederung der Karkasse und desto geringer die Seitenstabilität. Diese Anordung der Cordfäden verleiht dem Diagonalreifen eine stabile Seitenwand und somit eine erhöhte Widerstandsfähigkeit gegen mechanische Verletzungen. Gerade diese Robustheit ist es, die den Diagonalreifen für den Einsatz unter forstlichen Bedingungen qualifiziert.

Im Vergleich zu Radialreifen ist die Anzahl der Cordlagen bei Diagonalreifen insgesamt höher. Bei geringeren Geschwindigkeiten läuft ein Diagonalreifen weicher ab als ein Radialreifen. Dieses Verhältnis ändert sich bei Geschwindigkeiten über $40 \mathrm{~km} / \mathrm{h}$. Zudem kommt es zu einer verstärkten Vibration des Diagonalreifens bei Straßenfahrt. 


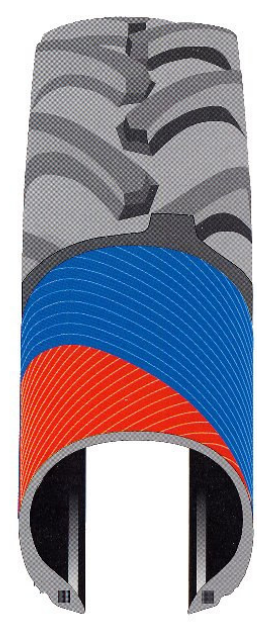

Abbildung 9: Aufbau von Diagonalreifen (BARUM, 2003)

\subsubsection{Radialreifen}

Bei den Radialreifen (Gürtelreifen) liegen die Cordfäden der Karkasse nebeneinander; sie kreuzen sich also nicht. Demnach verlaufen sie radial von Wulst zu Wulst, $d$. h. in einem Winkel von $90^{\circ}$ zur Fahrtrichtung (Abb. 10). Zwischen der Karkasse und der Lauffläche des Reifens wird zur Erreichung der Umfangsstabilität ein Gürtel aus mehreren Lagen Textil-, Stahl- oder Aramidgewebe im Winkel von etwa $20^{\circ}$ zur Fahrtrichtung angeordnet. Durch diesen Gürtel wird die Lauffläche beim Abrollvorgang nur sehr wenig verformt. Der Reifen federt an der Reifenflanke ein, so dass sich die Verformung hauptsächlich auf diese Walkzone ${ }^{2}$ beschränkt.

Radialreifen verfügen gegenüber vergleichbaren Diagonalreifen über einen geringeren Rollwiderstand und eignen sich besser für höhere Geschwindigkeiten. Der Gürtel sorgt für erhöhte Stabilität der Profilstollen beim Eingriff und erzielt eine höhere Traktion. Die Reifenflanken sind allerdings anfälliger gegen mechanische Verletzungen.

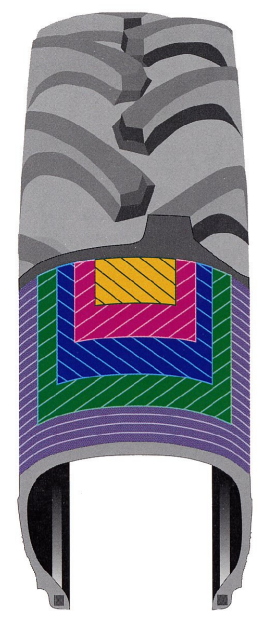

Abbildung 10: Aufbau von Radialreifen (BARUM, 2003)

\footnotetext{
2 Walken: Verformungsarbeit des Reifens beim Durchlaufen des Latsches. Unter einem Latsch versteht man die dynamische Kontaktfläche eines Reifens.
} 
Die Continental AG führte nach KRIEGER (2004) als erstes Unternehmen Radialreifen in der Forstwirtschaft ein. Mitte der 1990er Jahre wurden hierzu im Erzgebirge Maschinen mit dem Reifen AC $70 \mathrm{G}$ ausgerüstet und getestet. Nach positiven Testergebnissen erlangte der Reifen die Marktreife. Allerdings wurde mangels Nachfrage und als Folge einer unternehmenspolitischen und strategischen Entscheidung der Reifen wieder vom Markt genommen. Kurze Zeit später führte Michelin einen Radialreifen für die Forstwirtschaft mit Erfolg ein. Nach der Einführung von Radialreifen zogen andere Reifenhersteller nach, so z. B. Nokian im Jahre 2000.

\subsubsection{Reifenarten}

Die Reifenarten definieren sich über die Querschnittsform des Reifens. So unterscheidet man z. B. Ballon-Reifen, Super-Ballon-Reifen, Niederquerschittreifen, Super-Niederquerschnittreifen, 70er-, 60er- und 50er-Reifen ...). Bei den einzelnen Formen ist allein das Verhältnis von Reifenhöhe zu Reifenbreite verschieden. Die in der Forstwirtschaft verwendeten Reifenarten beschränken sich im Allgemeinen auf die Standard-/Normalquerschnittreifen und die Niederquerschnittreifen. Die Niederquerschnittreifen besitzen ein Verhältnis von Reifenhöhe zur Reifenbreite von höchstens 80 \% (ESSER ET AL., 2000). Die Reifenbreite wird nicht, wie bei den Standardreifen, in Zoll angegeben, sondern in Millimetern. Zudem wird die Reifenbezeichnung um das per Schrägstrich angeführte Verhältnis von Reifenhöhe zu Reifenbreite $(H / B)$ ergänzt (z. B. 600/65-34, d. h. $65 \%$ von der Reifenbreite 600 mm ergibt eine Reifenhöhe von $390 \mathrm{~mm}$ ). Bei den Standardreifen ist das Höhen-Breiten-Verhältnis hingegen nicht angeführt (z. B. 16.9-30). Eine Definition, ab welchem Querschnittsverhältnis von einem Standardreifen gesprochen wird, ist allerdings weder in Reifennormen noch von Seiten der Reifenhersteller eindeutig geregelt. Nach KRIEGER (2004) wird der Begriff „Standardreifen“zur Abgrenzung von verschiedenen Bereifungsalternativen (z. B. Breitreifen oder Niederquerschnittreifen) eines Fahrzeugs genutzt. Im landwirtschaftlichen Bereich gilt der Standardreifen als Grundbereifung und besitzt ein Höhen-Breiten-Verhältnis von 85 - 90 \%. Ähnliches bestätigt DISERENS (2002), der für einen Standard- bzw. Normalquerschnittreifen ein H/B-Verhältnis von $\geq 80 \%$ angibt. SCHULZ (1994) stellt fest, dass auch im Forst Standardreifen verwendet werden, die ein Verhältnis von Höhe zu Breite des Reifenquerschnitts von 70 - 80 \% aufweisen. Wird auf Grundlage dieser Aussagen ein Fazit gezogen, so besitzt ein Standard-/Normalquerschnittreifen i. d. R. ein Verhältnis von Reifenhöhe zu Reifenbreite von > $75 \%$. Ein weiteres Merkmal ist die zumeist in Zoll angegebene Nennbreite.

\subsubsection{Reifenkennzeichnung}

Es ist der Vorteil von technischen Normen oder Richtlinien, dass die verwendeten Begriffe genau definiert sind. Die Reifenkennzeichnung wird exemplarisch anhand eines Niederquerschnittreifens in Abbildung 11 dargestellt. 


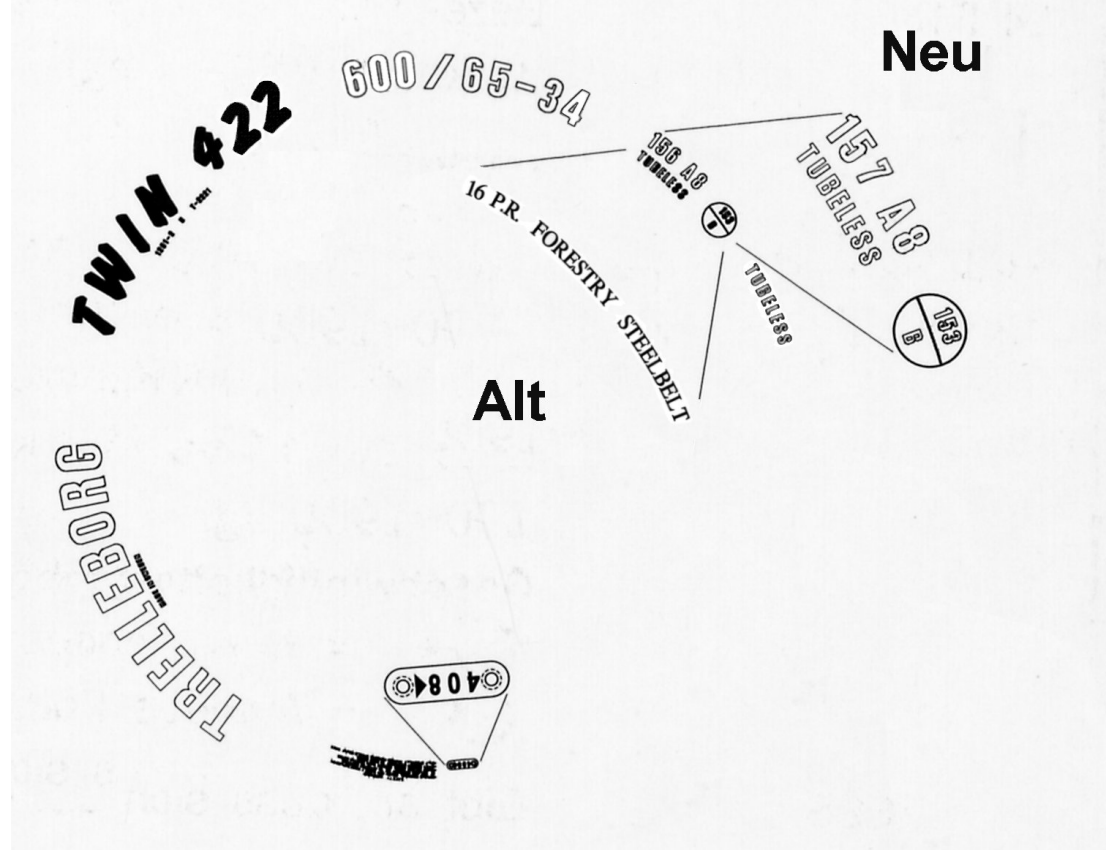

Abbildung 11: Reifenbezeichnung auf der Seitenwand von Forstreifen (verändert nach TRELLEBORG, 2003)

$\begin{array}{ll}\text { TRELLEBORG } & \text { Herstellername } \\ \text { TWIN } & \text { Produktname } \\ \mathbf{4 2 2} & \text { Profilbezeichnung } \\ 600 / 65-34 & \text { Bezeichnung der Reifendimension } \\ & 600=\text { Reifenbreite in } \mathrm{mm}\end{array}$

Definition: Ist die in der Reifengröße angegebene Breite eines aufgepumpten Reifens in Millimeter (oder bei Standardreifen in Zoll), der auf seiner theoretischen Felge montiert ist oder der gradlinige Abstand zwischen den Außenseiten der Seitenwände des aufgepumpten Reifens, eingeschlossen der Erhöhung durch die Beschriftungen, Verzierungen, Scheuerleisten oder Scheuerrippen (E.T.R.T.O., 2002).

65 = Reifenquerschnittsverhältnis $(H / B)$

Definition: Das Hundertfache des Verhältnisses zwischen der Querschnittshöhe und der Querschnittsbreite des unbelasteten Reifens auf seiner theoretischen Felge.

„-“ = Diagonalreifen

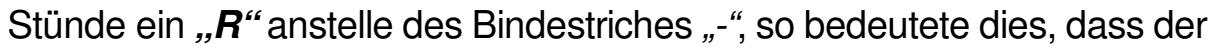
Reifen in Radialbauweise konstruiert ist: $\mathrm{R}=$ Radialreifen.

34 = Felgendurchmesser des Reifens in Zoll 
16 PR

157 A8

$153 \mathrm{~B}$

TUBELESS

408
Ply-Rating-Zahl (PR-Zahl)

Früher galt die PR-Zahl als Festigkeitsbezeichnung für die Karkasse. Die Angabe „16 PR" bedeutete, dass dieser Reifen aufgrund der Festigkeit seiner Karkasse ebenso belastet werden konnte, wie ein Reifen mit 16 Lagen Baumwollcord einer bestimmten Qualität. Die heutige Definition für die PR-Zahl nach E.T.R.T.O. versteht darunter „unterschiedliche Versionen (Tragfähigkeit/Luftdruck) von Reifen mit der gleichen Größenbezeichnung“. In Zukunft wird die PR-Zahl durch die Kombination LI/SI ersetzt werden.

Last-Index und Geschwindigkeitssymbol (in Kombination ist dies die aktuelle Kennzeichnung für Reifen)

157 = Last-Index $/$ Load-Index (LI)

Definition: Der Last-Index oder die Tragfähigkeitskennzahl ist ein nummerischer Code für die maximale Tragfähigkeit $(157 \stackrel{\wedge}{=} .125 \mathrm{~kg})$, die ein Reifen bei der durch das Geschwindigkeitssymbol spezifizierten Geschwindigkeit und bei den vom Reifenhersteller angegebenen Einsatzbedingungen hat. Eine Übersicht der Last-Indizes ist im Anhang 1 in der Abbildung 1.1 dargestellt.

A8 = Geschwindigkeitssymbol/Speed-Index (SI)

Definition: Der Speed-Index $(A 8 \stackrel{\wedge}{=} 40 \mathrm{~km} / \mathrm{h}$ ) gibt die Nenngeschwindigkeit an, mit dem der Reifen bei der durch den Load-Index angegebenen Last gefahren werden kann. Die Geschwindigkeitsindizes sind dem Anhang 1, Abbildung 1.2 zu entnehmen.

Zusatzkennzeichnung, die die Höchstlast bei der zulässigen Höchstgeschwindigkeit des Reifens angibt.

Schlauchlos

Gibt an, dass es sich um eine schlauchlose Ausführung des Reifens handelt.

Produktionsdatum (40 = Woche 40 und 8 = Jahr 1998).

Ab dem Jahr 2000 werden stattdessen 4 Ziffern angegeben, z. B. 0503 (05 = Woche 5 und 03 = Jahr 2003).

Weitere Reifenmaße bei variierenden Lasten sowie dazugehörige Definitionen finden sich im Anhang 1 in der Abbildung 1.3. 


\subsection{Flüssigkeitsfüllung in Forstreifen}

\subsubsection{Befüllung von Forstreifen}

Unterschiedliche Einsatzgebiete von selbstfahrenden Forstmaschinen erfordern häufig zusätzliches Gewicht, um z. B. die Antriebskräfte zu erhöhen. Mit einer zusätzlichen Auflast wird der Druck in der Aufstandsfläche des Reifens erhöht und der Schlupf kann bei gleichem Zugkraftbedarf (Zugkraft u. a. abhängig von der Normalkomponenten der Achslast) gemindert werden. Gleichzeitig kommt es zu einer Senkung des Schwerpunktes und zu einer Verbesserung der Standsicherheit (v. a. am Hang). Eine Erhöhung der Radlasten kann entweder durch an Rädern (und/oder Fahrgestell) montierte Gewichte oder durch die Füllung der Reifen mit Flüssigkeit realisiert werden.

Nach Angaben in der bisherigen Literatur (s. hierzu GERDSEN, 1997; BERNHARD UND SCHLOTTER, 2001; WEISE, 2002; TRELLEBORG, 2003) verringert die Flüssigkeitsfüllung das komprimierbare Reifenvolumen und erhöht die Federsteifigkeit (Reifen werden starrer und sind weniger elastisch). Das führt vielfach zu einer Veränderung der dynamischen Eigenschaften, wie z. B. einer Verschlechterung des gesamten Schwingungsverhalten der Forstmaschine. Überdies besteht die Gefahr einer Reifenbeschädigung, die sich durch kurzzeitige (starke) Innendruckerhöhung bei Überfahrten von Hindernissen ergeben kann.

Um dennoch bei einem flüssigkeitsgefüllten Reifen die erforderliche Flexibilität zu gewährleisten, sollten maximal ca. 70 - 75 \% des Volumens mit Flüssigkeit gefüllt werden (Abb. 12). Diesen Füllgrad erreicht man, wenn das Ventil in seiner höchsten Position über Grund steht und der Reifen bis zum Ventilfuß gefüllt wird.

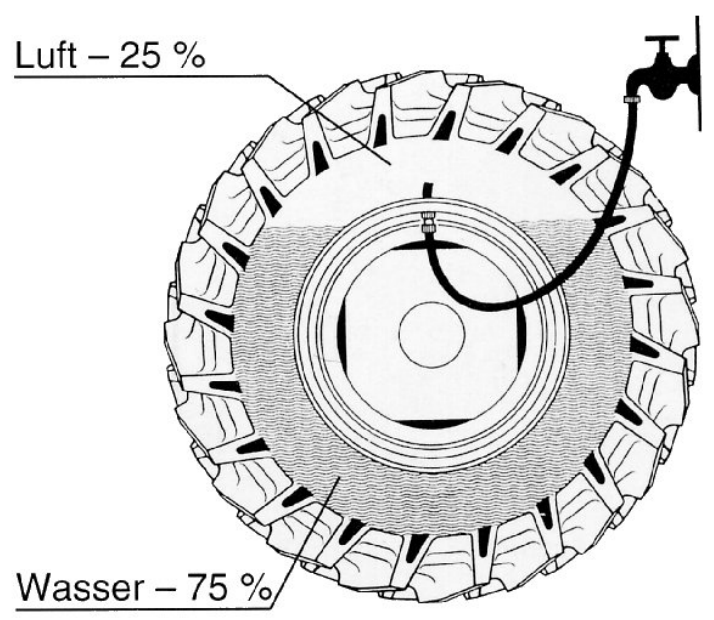

Abbildung 12: Flüssigkeitsbefüllung von Reifen (BARUM, 2003)

Zur Befüllung muss anstelle dieses Ventileinsatzes ein kombiniertes Flüssigkeitsfüll- und Entleerventil auf den Ventilschaft geschraubt werden. In diesem Zusammenhang ist häufig von der so genannten "Hanauer Maus“ die Rede. Dies ist eine von der EHA Ventilfabrik (Mühlheim/Main) vertriebene Vorrichtung zur Flüssigkeitsbefüllung. Die EHA Ventilfabrik wurde inzwischen durch die Alligator Ventilfabrik GmbH (Giengen/Brenz) übernommen. Daher wird in 
Zukunft die „Hanauer Maus“durch den auf gleichem Funktionsprinzip basierenden „Waterboy“ abgelöst (Abb. 13). Die kombinierten Flüssigkeitsfüll- und Entleerventile besitzen eine seitliche Öffnung, durch die Luft während des Befüllvorgangs entweichen kann.

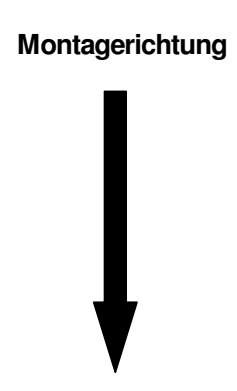

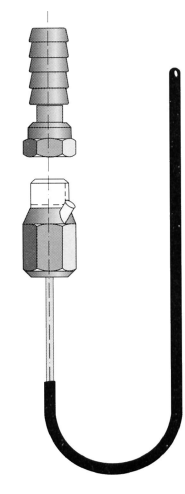

Hanauer Maus

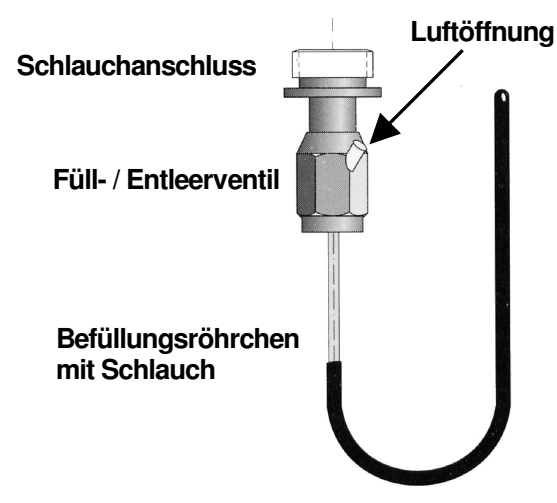

Waterboy

Abbildung 13: Hanauer Maus und Waterboy (BARUM, 2003)

\subsubsection{Wasser bzw. Frostschutzlösung}

Vielfach wird in der forstlichen Praxis von „Wasser“ als Füllmedium gesprochen. Das trifft jedoch nur für Regionen zu, in denen keine Frostgefahr besteht. Unter mitteleuropäischen Klimabedingungen ist vielfach im Winter mit anhaltenden Temperaturen unter dem Gefrierpunkt zu rechnen, so dass die Reifenhersteller die Verwendung einer Frostschutzlösung bei der Befüllung von Reifen empfehlen. In diesem Fall werden dem Wasser häufig Calciumchlorid $\left(\mathrm{CaCl}_{2}\right)$ oder auch Magnesiumchlorid $\left(\mathrm{MgCl}_{2}\right)$ in einem dem erwarteten Temperaturbereich angegebenen Mischungsverhältnis zugegeben. Diese Salzlösungen haben jedoch bei schlauchlosen Reifen den Nachteil, dass sie den Felgenstahl angreifen und damit verstärkt Korrosion am Material auftritt. Von Seiten der Reifenhersteller wird deshalb bei der Flüssigkeitsbefüllung - generell bei Verwendung von schlauchlosen Reifen - verstärkt auch Frostschutzmittel auf Basis von Ethylenglykol $\left(\mathrm{C}_{3} \mathrm{H}_{6} \mathrm{O}_{2}\right)$ empfohlen. Dieser Frostschutzzusatz, in der Praxis oft nur als „Glykol“ bezeichnet, findet sich vor allem in geschlossenen Motorkühlsystemen.

Alle Frostschutzzusätze haben im Vergleich zum Wasser ein höheres spezifisches Gewicht. Infolgedessen erhöht sich das Gesamtgewicht des befüllten Forstrades. Die Tabelle 1 zeigt, dass sich die beiden Frostschutzzusätze (in Abhängigkeit von den erwarteten Temperaturen) hinsichtlich der Gesamtmasse der Lösungen unterscheiden und eine durchschnittliche Gewichtsdifferenz von 10 \% festzustellen ist. Das Gewicht der Flüssigkeit in den befüllten Reifen muss bei der Bestimmung des Gesamtgewichtes berücksichtigt werden (TRELLEBORG, 2003). 
Tabelle 1: Mischungsverhältnisse von Frostschutzzusätzen in Abhängigkeit der Temperatur (verändert nach Trelleborg, 2003)

\begin{tabular}{|c|c|c|c|c|c|c|c|}
\hline \multirow{3}{*}{$\begin{array}{c}\text { Temperatur } \\
{ }^{\circ} \mathrm{C}\end{array}$} & \multicolumn{3}{|c|}{ Calciumchlorid } & \multicolumn{4}{|c|}{ Glykol } \\
\hline & \multirow{2}{*}{$\frac{\mathrm{CaCl}_{2}}{\boldsymbol{k g}}$} & \multirow{2}{*}{$\frac{\text { Wasser }}{I}$} & \multirow{2}{*}{$\frac{\text { Dichte }}{\text { kg/l }}$} & \multicolumn{2}{|c|}{ Glykol } & \multirow{2}{*}{$\frac{\text { Wasser }}{I}$} & \multirow{2}{*}{$\begin{array}{c}\text { Dichte } \\
\boldsymbol{k g} / \mathbf{l}\end{array}$} \\
\hline & & & & $I$ & kg & & \\
\hline-10 & 0,14 & 0,95 & 1,09 & 0,23 & 0,26 & 0,77 & 1,03 \\
\hline-20 & 0,24 & 0,90 & 1,14 & 0,33 & 0,37 & 0,67 & 1,04 \\
\hline-30 & 0,36 & 0,83 & 1,19 & 0,43 & 0,48 & 0,57 & 1,05 \\
\hline-40 & 0,49 & 0,76 & 1,25 & 0,53 & 0,59 & 0,77 & 1,06 \\
\hline
\end{tabular}

Zur Flüssigkeitsbefüllung sollten möglichst die Hersteller der verwendeten Reifen bezüglich der Empfehlungen für Frostschutzlösungen befragt werden. Dies gilt insbesondere auch für die umweltgerechte Entsorgung der Flüssigkeiten nach dem Ablassen aus dem Reifen, da diese dem Sondermüll zuzuordnen sind. 


\section{Einleitung}

\subsection{Problemstellung}

Etwa ein Drittel der Fläche der Bundesrepublik Deutschland ist von Wald bedeckt. Waldböden kommt angesichts immer intensiverer Inanspruchnahme der nichtbewaldeten Böden durch Landwirtschaft, Industrie, Verkehr und Besiedlung eine wachsende Bedeutung zu. Als weitgehend naturnahes Ökosystem übernehmen sie wichtige Ausgleichs-, Speicher- und Pufferfunktionen. Für die Forstwirtschaft ergibt sich daraus die Verpflichtung, der Schonung des Bodens im Zuge der Waldbewirtschaftung eine hohe Priorität einzuräumen. Vorwürfe werden vorrangig gegen hochmechanisierte Verfahren in der Holzernte erhoben, da hier schwere Maschinen eingesetzt werden, die offensichtlich Böden stark belasten und Strukturveränderungen verursachen. Hohe Eigenmassen der Maschinen plus Zuladung ( $5 \mathrm{t}$ bis $30 \mathrm{t}$ ), häufiges Befahren und ein aus Bodenstruktur und Wassergehalt herrührender ungünstiger Bearbeitungszeitpunkt verstärken die negativen Auswirkungen dieser Belastung.

Eine Befahrung des Waldbodens mit radgetriebenen Forstmaschinen während der Holzernte erfolgt seit nunmehr ca. 50 Jahren. Dabei hat die technische Entwicklung von Forstmaschinen kontinuierlich zugenommen. Leichte Schlepper lösen in den 50er Jahren des letzten Jahrhunderts die bis dahin mit dem Pferd durchgeführte Holzbringung langsam ab (ROGALL, 1968). Nach HAMBERGER (2002) werden in den 1960er Jahren etwa $80 \%$ des Holzes mit landwirtschaftlichen Schleppern gerückt. Die Stämme werden direkt angefahren und auf kürzest möglichem Weg zur Waldstrasse transportiert. In den 1970er Jahren setzen sich FeinerschlieBungsnetze durch. Erste Forstspezialschlepper (Skidder) werden entwickelt. Die eingesetzten landwirtschaftlichen Schlepper werden schwerer und sind mit Polterschild und Funkseilwinde sowie weiteren aufwendigen Forst-Zusätzen ausgerüstet. In den 1980er Jahren setzen sich gegenüber landwirtschaftlichen Schleppern, Forstspezialschlepper und Tragschlepper durch, die eine bessere Geländegängigkeit und ein größeres Transportvolumen aufweisen. Damit nimmt die Belastung des Bodens zu. Die negativen Auswirkungen werden erkannt und verstärkt wissenschaftlich untersucht (HILDEBRAND, 1981; HILDEBRAND UND WIEBEL, 1982; BENECKE, 1982; BREDBERG UND WÄSTERLUND, 1983; LÖFFLER, 1983; BECKER ET AL. 1986; LÖFFLER, 1986; ZANDER, 1988). Ab Mitte der 1980er Jahre werden erste Harvester aus Skandinavien erprobt und etablieren sich. Ausschlaggebend für den Durchbruch der Harvestertechnologie ist deren erfolgreicher Einsatz zur Aufarbeitung der Sturmwürfe von „Vivian“ und „Wiebke“1990 (OHRNER ET AL., 2003). Die schnellere und rationelle Bereitstellung des Holzes und die damit verbundene Kostenreduktion bewirkt einen rapiden Anstieg der in Deutschland eingesetzten Forstspezialmaschinen im Laufe der neunziger Jahre des zurückliegenden Jahrhunderts. Nach einer von EBEL UND JACKE (2003) durchgeführten Erhebung ist im Jahr 2002 deutschlandweit von einem Bestand von ca. 950 Harvestern und ca. 1.800 Forwardern auszugehen.

Die Entwicklung seit den achtziger Jahren des letzten Jahrhunderts lässt einen enormen Anstieg des Mechanisierungsgrades in der Forstwirtschaft erkennen, der ausschließlich auf wirtschaftliche Zwänge der Waldbesitzer zurückzuführen ist, $u$. a. verursacht durch hohe Perso- 
nalkosten und stagnierende Holzpreise (MATTHIES ET AL., 1995; HAMBERGER, 2002; WEHNER, 2002; ZIESAK, 2004). Produktivitätsaspekte ließen dabei die Maschinen in Leistung und Größe beständig zunehmen (FORBRIG, 2000; WEHNER, 2002). Der gezielte Einsatz von leistungsfähigen, radgetriebenen Forstspezialmaschinen gestattet die Kosten der jeweiligen Arbeitsverfahren gering zu halten. Die erhöhte Effizienz dieser Maschinen ist aber oft mit einer verstärkten mechanischen Belastung des Bodens verbunden.

Während bei Befahrung wassergesättigter Böden überwiegend das sog. Bodenfließen mit starker Gleisbildung auftritt (vorwiegend bei Böden mit hohem Tonanteil), kommt es bei mechanischer Belastung ungesättigter Oberböden durch die vom Fahrzeug über die Räder auf den Boden ausgeübten Kräfte zu entsprechenden Drücken, die vorrangig zu Bodenverdichtungen führen (BECKER ET AL., 1986; LÖFFLER, 1986). In beiden Fällen wird die Tragfähigkeit des Bodens überschritten. Die Tragfähigkeit hängt dabei maßgeblich von der Bodenart und der Bodenfeuchte ab. Als Ausdruck einer überschrittenen Bodentragfähigkeit bleiben vom Reifen Spuren zurück. Hierbei unterscheidet ABEELS (1983) drei Fälle:

1. keine seitliche Bodenquetschung, jedoch vertikale Verdichtung

2. seitliche Ausquetschung, jedoch keine vertikale Verdichtung

3. seitliche Ausquetschung und vertikale Verdichtung.

Von den drei Komponenten des Bodens - den Festteilchen, dem Wasser und der Luft - lassen sich Festteilchen und Wasser nicht komprimieren. Eine wesentliche bodenphysikalische Veränderung, die durch ein Befahren des Bodens ausgelöst wird, ist die Erhöhung der Lagerungsdichte, d. h. das Zusammenpressen der Bodenaggregate (HOFMANN, 1989; MATTHIES, 1994; DEUTSCHER VERBAND FÜR WASSERWIRTSCHAFT UND KULTURBAU, 1997). Eine Verdichtung geht deshalb nahezu ausschließlich zu Lasten des luftgefültten Porenvolumens. Zudem wird die Porenkontinuität (Vernetzung der Poren untereinander) zunehmend unterbrochen, wodurch ein Austausch von Flüssigkeiten und Gasen erschwert wird. Zusätzlich können durch Abscherung Porensysteme und damit die Oberflächenversiegelung unterbrochen werden.

Die durch eine Verdichtung des Bodens verursachten Strukturveränderungen beeinflussen zudem die Rhizosphäre und wirken sich über das Wurzelwachstum auf das gesamte Pflanzenwachstum aus. So kann es zu einer Verringerung des für die Wurzeln benötigten Sauerstoffs und zu einer Anreicherung des produzierten Kohlendioxids kommen. Der Flüssigkeitsaustausch ist vermindert und führt zu einer eingeschränkten Versorgung mit Wasser und Nährstoffen und zu einer reduzierten Entsorgung von Zellabfallstoffen im Wurzelbereich. Gleichzeitig wird durch eine Verdichtung des Bodens die Durchwurzelung der komprimierten Bodenpartien vermindert. Die Folgen für den Bestand sind eine geringere Feinwurzeldichte, ein eingeschränktes Pflanzenwachstum sowie Stabilitätsverluste. Größere Wurzelverletzungen begünstigen auch Pilzinfektionen, die ebenfalls zur Minderung der Stabilität führen können. In der forstlichen Literatur ist eine Vielzahl von Untersuchungen zu diesem Themenbereich zu finden (BREDBERG UND WÄSTERLUND, 1983; SCHACK-KIRCHNER UND HILDEBRAND, 1993; POTSCH, 1996; WILPERT, 1998; HILDEBRAND ET AL., 2000; GAERTIG ET AL., 2001). 
Untersuchungen von LÖFFLER (1986), BECKER ET AL. (1989) und HOFMANN UND BECKER (1990) kamen zu dem Ergebnis, dass bereits die erste Überfahrt schwerwiegende und unter Umständen andauernde Bodenstrukturveränderungen verursacht.

Die nachhaltige Bodenfruchtbarkeit stellt für die Forstwirtschaft eine der wichtigsten zu erhaltenden Funktionen dar. Neben dem Eigeninteresse des Erhalts dieser natürlichen Ressource durch die Forstwirtschaft besteht ein ausgeprägtes umweltpolitisches Engagement der Bevölkerung. Die in der Gesellschaft gewachsene ökologische Orientierung nimmt das Geschehen im Wald kritisch wahr (SCHLAGHECK, 2000; SUDA UND BECK, 2001). So werden Böden in der Gesellschaft immer häufiger als Kulturgut interpretiert, dessen Funktion und letztlich Existenz durch politisch motivierte Maßnahmen geschützt werden müssen. Wirtschaftende Aktivitäten auch der Forstbranche - geraten im Zuge solcher Diskussionen rasch in die Rolle der „Zerstörer des Kulturgutes Boden“, und es gilt, solchen Vorwürfen vorzubeugen.

Viele Landesforstverwaltungen (und auch größere Privat-Forstbetriebe) fordern deshalb ökologische Bewertungskriterien für die Bodenpfleglichkeit der Forstmaschinen. Des Weiteren verlangen die umweltbewusste Gesellschaft, Kunden und Marktpartner der Forstwirtschaft, Umweltverbände und Verbraucher inzwischen einen glaubwürdigen und schlüssigen Nachweis für eine nachhaltige Waldbewirtschaftung in Deutschland, zu der auch der Schutz des Bodens gehört (s. hierzu HAMBERGER, 2002; VOLZ UND WINKEL, 2003). Dies geschieht zum einen direkt durch gesetzliche Vorgaben und zum anderen indirekt auf ökonomischem Weg durch das Instrument der Zertifizierung.

Es traten gesetzliche Regelungen in Kraft, die neben Wasser und Luft ausdrücklich das Umweltgut "Boden“ unter Schutz stellen. Hier ist das BUNDESBODENSCHUTZGESETZ (BBODSCHG, 1998) sowie die Neuregelung des BUNDESNATURSCHUTZGESETZES (BNATSCHG, 1998) zu nennen. Dabei lassen sich einige der in § 17 (2) BBodSchG angeführten Grundsätze zur „Guten fachlichen Praxis in der Landwirtschaft"auch auf die Forstwirtschaft übertragen. Insbesondere handelt es sich um die Grundsätze zum Erhalt der Bodenstruktur, die Vermeidung von Bodenverdichtung, die Vorkehr gegen Bodenabtrag (Erosion) und den Erhalt des standorttypischen Humusgehaltes. Wenngleich die aufgeführten Grundsätze für die Forstwirtschaft nicht verbindlich sind (GIESEN, 2003), so werden hier doch Maßstäbe gesetzt, denen sich die forstliche Praxis auf lange Sicht nicht verschließen sollte (MATTHIES, 2000, VOLZ UND WINKEL, 2003). Vor dem Hintergrund dieser Forderungen des Gesetzgebers zum Schutz des Bodens und der zunehmenden Mechanisierung in der Forstwirtschaft, haben die zuständigen Behörden und Länder Richtlinien erlassen, die den vor Ort Verantwortlichen bei der praktischen Ausübung des Bodenschutzes im Wald helfen sollen. MATTHIES (2000) kommt zu dem Schluss, dass keine dieser Handlungsanweisungen geeignet ist, den Bodenschutz in der gesellschaftlich gestellten Anforderung zu gewährleisten.

Der Schutz des Bodens wird ebenfalls von den zwei bedeutenden derzeit vergebenen Zertifizierungen für die Waldbewirtschaftung in Deutschland gefordert. Vorrangiges Ziel des Forest Stewardship Council (FSC) und des Programme for the Endorsement of Forest Certification Schemes (PEFC) ist es, eine umweltgerechte, sozialverträgliche und wirtschaftlich tragfähige Bewirtschaftung der Wälder zu fördern. Von beiden Systemen ist der Schutz des Bodens explizit in deren Leitlinien verankert und muss folglich von den zertifizierten Waldeigentümern berücksichtigt und in die Praxis umgesetzt werden (FOREST STEWARDSHIP COUNCIL, 2004; 
PROGRAMME FOR THE ENDORSEMENT OF ForESt CERTIFICATION SCHEMES, 2005). FSC verweist darauf, dass eine Befahrung des Waldbodens ausschließlich auf Rückgassen erfolgt und jegliche flächige Befahrung unterbleibt. PEFC gestattet bei besonderen topografischen Situationen eine Abweichung vom Feinerschließungsnetz, wenn dadurch Schäden an Boden und Bestand vermieden werden können. Beide Systeme unterstreichen, dass die Funktion der Rückegasse durch die Wahl von geeigneten Arbeitsgeräten und deren Ausrüstung (z. B. Breitreifen, Gleisketten etc.), optimaler Planung und Logistik zur Reduktion der Überfahrten, Berücksichtigung witterungsbedingter Einflüsse sowie Stabilisierung der Rückegassen durch Reisigauflagen erhalten bleibt und damit eine bodenschonende Holzbringung garantiert. Darüberhinaus fordert PEFC, das Befahren zusätzlich zur Holzernte (Bodenbearbeitung, Pflanzung, Saat) auf ein unbedingt erforderliches Ausmaß zu begrenzen und bei verdichtungsempfindlichen Böden die Befahrung bodenschonend (geringer Reifeninnendruck, geringe Radlast und möglichst Breitreifen) zu gestalten.

Die Forsttechnik befindet sich nach ZIESAK (2004) in einem Konfliktfeld, welches einerseits durch die in der Waldbewirtschaftung eingesetzten Maschinen und den damit verbundenen Belastungen für den Boden, andererseits durch immer weitergehende Reglementierungen und den Einfluss der Öffentlichkeit determiniert wird.

Dementsprechend sind die Befahrung des Waldbodens und die damit verbundenen Auswirkungen auf seine Struktur und ökologische Funktionalität seit mehr als 20 Jahren Thema wissenschaftlicher Untersuchungen.

MATTHIES (2000) stellt hierzu fest, dass bei einer geeigneten Verschneidung der Steuerungsgrößen eine Befahrung des Waldbodens unter Wahrung seiner ökologischen Funktionen möglich ist. Das erlangte Wissen wurde $u$. a. in Bodenschutzkonzepten ${ }^{3}$ oder Verordnungen der Landesforstverwaltungen umgesetzt.

Während aber die bodenphysikalischen Veränderungen in Abhängigkeit bodenmechanischer Kenngrößen und Bodenfeuchtezustände relativ umfassend publiziert sind (BOLLING, 1986; DürR ET AL., 1995; MATTHIES ET AL., 1995; MATTHIES UND KREMER, 1999), offenbaren sich Kenntnisdefizite hinsichtlich der Schnittstelle „Reifen-Boden“. Der Reifen ist hierbei die Kontaktstelle der Maschine mit dem Boden, der die jeweilige Gewichtskraft überträgt.

Kontaktflächendrücke wurden (und werden) in den unterschiedlichsten Anwendungsbereichen aus der radbezogenen Auflast und der relativ einfach zu bemessenden Kontaktfläche (zumeist auf hartem Untergrund) statisch berechnet:

$$
\begin{aligned}
\text { Druck } & =\text { Kraft } / \text { Fläche } \\
p & =\mathrm{F} / \mathrm{A}
\end{aligned}
$$

Veröffentlichte Kontaktflächendrücke (z. B. in den Beurteilungen selbstfahrender Maschinen durch den Forsttechnischen Prüfungsausschuss (FPA) des Kuratoriums für Waldarbeit und Forsttechnik (KWF)) beruhen auf formellen Berechnungen, die systematisch zu zu niedrigen

\footnotetext{
${ }^{3}$ Einen umfassenden Überblick der Bodenkonzepte, die das Ziel haben, die Belastungssituationen betroffener Böden zu entschärfen, findet sich in ZIESAK (2004).
} 
Werten führen (GERDSEN UND GRAUPNER, 1998, KURATORIUM FÜR WALDARBEIT UND FORSTTECHNIK, 2003).

Einen in jeder Hinsicht umfassenden Überblick einer Sammlung gängiger Flächen- und Druckermittlungsverfahren für land- und forstwirtschaftliche Reifen einschließlich ihrer Bewertung hat SAARILAHTI (2002) erstellt. Dabei kommt er zu dem Fazit, dass die Berechnungen der Aufstandsfläche und des Kontaktflächendruckes irreführend sind, da sie unrealistisch niedrige Werte für den Kontaktflächendruck ausweisen. Für SAARILAHTIS (2002) Vergleich von über 20 Ansätzen zur Berechnung des Kontaktflächendruckes konzentrierte er sich schwerpunktmäßig auf den Nominal Ground Pressure (NGP) und den Mean Maximum Pressure (MMP).

NGP, als „SkogForsk-Formel“ oder „Swedish Formula"bekannt (s. hierzu MIKKONEN und WUOLIJOKI, 1975), gilt als Standardmodell zur Berechnung von Aufstandsflächen und unter Einbeziehung der Radlast zur Ermittlung des Kontaktflächendruckes. Da dieses theoretische Modell die maximale Aufstandsfläche berechnet, trifft es Aussagen zum hypothetischen minimalen Kontaktflächendruck. Beim MMP-Modell wird darauf abgezielt, den durchschnittlichen Maximaldruck zu ermitteln. Das Modell wurde von RowLAND (1972) entwickelt und hat den Vorteil, dass zusätzliche Parameter, wie z. B. Reifenfülldruck, Radlast und Einfederung, einbezogen werden. Diese Ausgangsformeln zur Berechnung des Kontaktflächendruckes über die Aufstandsfläche (NGP) bzw. zur Berechnung des Maximaldruckes (MMP) bildeten die Basis zahlreicher weiterer Modelle. SAARILAHTI (2002) stellt hierzu fest, dass NGP-Modelle für Mobilitätsuntersuchungen, wie Schub-, Zug- und Rollwiderstände von Rädern, geeignet sind. Für Untersuchungen von Bodenstrukturveränderungen empfiehlt er aber Modelle, die auf dem MMP fußen.

Ein ausschlaggebendes Kriterium bei den von SAARILAHTI (2002) zusammengefassten Formeln ist die Größe der Aufstandsfläche. Konstruktionsbedingte Elemente beeinflussen im Besonderen Maße das Verformungsverhalten des Reifens. Dies führt dazu, dass sich die Größe und Form der Aufstandsfläche in Abhängigkeit der auf einen Reifen wirkenden Kräfte und dem Verhalten des Untergrundes ändert. Hinsichtlich ihrer Erhebung gibt es eine Vielzahl von Verfahren, wenn sie nicht theoretisch ermittelt wurden. Zumeist wurden die Aufstandsflächen in Form von „Stempelabdrucken " oder mit Kreidepulver markiert (übertragen auf eine Folie auch Hüllkurve genannt) aufgenommen und ausplanimetriert (SCHWANGHART, 1993; GERDSEN UND GRAUPNER, 1998). Die Größe dieser Flächen steht in direktem Zusammenhang mit der Höhe des in den Formeln ausgewiesenen mittleren Kontaktflächendruckes. Dies hieße, je größer die Fläche, desto niedriger der Druck. Ein Vergleich dieser Flächen untereinander galt in vielen Fällen als Bewertung des auftretenden Kontaktflächendruckes und als Begründung eines bodenschonenderen Einsatzes. Dabei ist die Aufstandsfläche allein nicht geeignet, Aussagen über die Bodenschonung einzelner Reifen zu treffen. MATTHIES UND ZIESAK (2001) stellten fest, „dass dieser nummerisch abgeleitete Bodendruck nicht dem tatsächlich unter einem Reifen auftretenden Druck entspricht, da der Reifen als verformbares Medium offenbar die auf inn wirkende Radlast in seinem Kontaktbereich nicht gleichmäßig in den Boden weiterleitet." Ein Vergleich von verschieden breiten Reifen unter ansonsten gleichen

\footnotetext{
${ }^{4}$ Der an der Lauffläche mit Druckerfarbe bestrichene Reifen wird mit einer definierten Radlast auf einen harten Untergrund gedrückt. Zwischen Reifen und hartem Untergrund liegt ein Bogen Papier. Durch regelmäßiges drehen und andrücken des Reifens erhält man seine Aufstandsfläche, die als „Stempelabdruck“ bezeichnet wird.
} 
Randbedingungen zeigte annähernd gleiche Kontaktflächengrößen. Diese Untersuchung macht deutlich, dass bei annähernd identischen Aufstandsflächen der breitere Reifen keinen Vorteil bezüglich des Bodendruckes und damit der Bodenschonung bietet.

Nicht die Aufstandsfläche an sich, sondern die Druckverteilung auf der Kontaktfläche des Reifens erlaubt erst quantifizierte Angaben zum bodenschonenden Einsatz selbstfahrender Arbeitsmaschinen und deren Bereifungen. Für die in der forstlichen Praxis eingesetzten Reifen existieren solche Untersuchungen nicht. Im Rahmen des Projekts „Druckverteilung auf Kontaktflächen unter Forstreifen oder Pressure Allocation on Contact Areas under Forest Tires (PrAIICon)"soll diese Druckverteilung erforscht werden. Insbesondere die Frage, wie sich die Druckzonen in der Kontaktfläche ausprägen, steht im Vordergrund und gilt es zu ergründen. Dabei besteht die Grundannahme darin, dass sich eine Zone höchsten Druckes unterhalb der Radnabe befindet und somit der Schwerpunkt der Druckbelastung vom Zentrum der Kontaktfläche mit stetiger Abnahme zur Peripherie verläuft (Abb. 14).

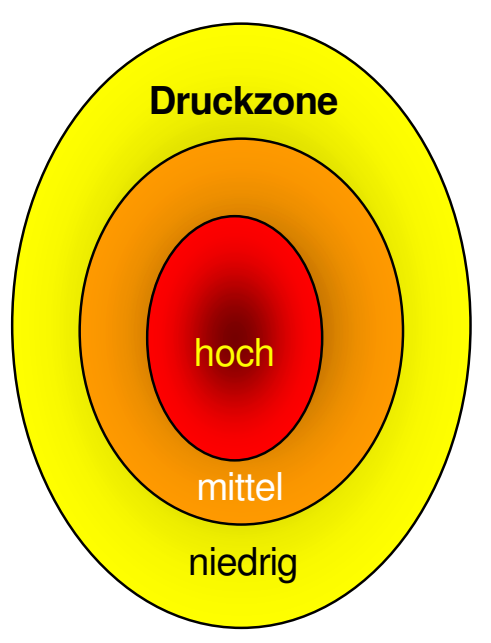

Abbildung 14: Hypothetische Kontaktflächendruckverteilung

\subsection{Zielsetzung}

Für die vollständige bodenökologische Betrachtung bezüglich des Einsatzes von Forstmaschinen ist es notwendig, die Schnittstelle „Reifen-Boden“ näher zu betrachten. Dafür schien die wissenschaftlich-systematische Erforschung der Druckverteilung auf den Kontaktflächen unter Forstreifen notwendig. Hierzu soll eine Marktanalyse der in der forstlichen Praxis auf den Maschinen montierten Reifen erfolgen. Parallel dazu wird eine geeignete Messtechnik entwickelt, um die Kontaktflächendruckverteilungen auf hartem und weichem Untergrund zu ermitteln. Hauptziel dieser Arbeit ist die sich anschließende Analyse und Interpretation der Daten. Dafür werden zwei Schwerpunkte gesetzt:

1. Statistische Analyse auf den Grad der Korrelation zwischen Parametern des Rades, wie Radlast, Fülldruck, Reifenbreite, Raddurchmesser, Tragfähigkeit, Bauweise und Reifenprofilierung. 
2. Ermittlung von Gesetzmäßigkeiten, um in Zukunft Kontaktflächendruckverteilungen unter den Forstreifen modellieren zu können.

Mit diesen empirisch ermittelten Kontaktflächendrücken besteht die Möglichkeit eines realen Ansatzes für den bodenökologischen Vergleich von Forstreifen, der bislang ausschließlich auf theoretischen Formeln basiert. 


\section{Marktanalyse der auf selbstfahrenden Forstmaschinen mon- tierten Reifen}

\subsection{Erhebungsmethodik}

Um eine repräsentative Übersicht der zurzeit in Deutschland verbreiteten Forstmaschinen und deren Bereifung hinsichtlich Hersteller, Dimensionen, Karkassenfestigkeit und Profilierung zu erhalten, wurde im Jahr 2002 eine Marktanalyse durchgeführt, deren Resultate im Folgenden vorgestellt werden.

Beginnend mit einem intensiven Literaturstudium erfolgte eine Sichtung von Fachbüchern, Fachzeitschriften, Dissertationen etc.. In Bezug auf die Frage der in Deutschland auf selbstfahrenden Forstmaschinen eingesetzten Reifen lieferte dieses Material nicht genügend Informationen.

Zum Erhalt eines ersten Überblicks wurden systematisch die Gebrauchtmaschinen-Annoncen der Zeitschriften „Forst und Technik" und „Forstmaschinenprofi“" der Jahrgänge 2000 bis 2002 (einschließlich des Monats Februar) sowie das Internet nach den Maschinengruppen Harvester, Forwarder, Schlepper und Landwirtschaftliche Schlepper mit Forstausrüstung sowie deren Bereifung ausgewertet.

Die in den Kleinanzeigen zu den Reifen ausgeführten Angaben beschränkten sich hauptsächlich auf die Nennbreite und den Felgendurchmesser, teilweise wurden Angaben zum Höhen/Breiten-Verhältnis, zu der PR-Zahl und zum Profil gemacht. In vielen Fällen fehlten Reifenangaben gänzlich. Diese fragmentarischen Daten wiesen die für eine umfassende Auswertung benötigten Informationen zu den einzelnen Reifendimensionen (Nennbreite, Höhen-/BreitenVerhältnis, Felgendurchmesser, Load-Index/PR-Zahl, Bauweise (diagonal/radial), Profil und Reifenhersteller) nicht auf. Auf eine weitere Bearbeitung und Auswertung wurde deshalb verzichtet.

Die Firmenniederlassungen der Forstreifenhersteller in Deutschland befriedigen primär die Nachfrage auf dem Ersatzreifenmarkt (Aftermarket). Aussagen über die Einsatzhäufigkeiten der einzelnen Forstreifen waren demzufolge sehr lückenhaft. Großen Forstreifenherstellern, wie z. B. Nokian oder Trelleborg, war es ebenfalls nicht möglich, die Anzahl der Forstreifen, die nach Deutschland exportiert wurden, zu benennen. Nach Aussage einiger Firmenvertreter führe eine umfassende Forstreifenerhebung nur durch eine direkte Befragung jener Unternehmen, die für die Montage der Reifen beim Verkauf der Maschinen verantwortlich zeichnen, zum Erfolg. Demnach obliegt grundsätzlich die Entscheidung über die auf Forstmaschinen zu montierenden Reifen entweder dem Maschinenproduzenten oder (seltener) dessen Handlungsbeauftragten (Generalimporteur) (MÜLLER, 2002). Die Ausrüstung erfolgt nach getesteten Standards, nicht selten auch nach Kundenwunsch in einem vom Maschinenhersteller gesetzten Auswahlspektrum.

Vor diesem Hindergrund (Verifizierung der Aussagen und Erhebung der aus dem Ausland importierten und in Deutschland produzierten Forstspezialmaschinen sowie deren Bereifung), 
wurde in einem ersten Schritt ein Fragebogen entworfen und an ausgewählte Forstmaschinenhersteller und Generalimporteure im In- und Ausland versandt.

Im Einzelnen handelte es sich um die 22 nachfolgend (in alphabetischer Ordnung) benannten Unternehmen:

\author{
Dasser Spezialfahrzeuge $\mathrm{GmbH}$ \\ DaimlerChrysler / MB Trac \\ Ferdinand Müller OHG / Fahrzeugbau - Forsttechnik \\ Hans-Hasso Grimm Forst- und Baumschulentechnik (LKT, Silvatec-Harvester, FMA, \\ NFB) \\ HSM Hohenloher Spezial-Maschinenbau GmbH \& Co. \\ Hübinger GmbH (bis 2000 Vertrieb von John Deere-Skiddern) \\ John Deere Vertrieb / Deutschland \\ Kopa Forstmaschinen-Handels-und-Reparatur-GmbH (Rottne) \\ Nuhn GmbH \& Co. KG (Timberjack) \\ Partek Forest GmbH (Valmet) \\ Pfanzelt Maschinenbau GmbH (rüstet ausschließlich Schlepper der Firma Fendt \\ aus) \\ Ritter Maschinenfabrik / A. Ritter \& Söhne GmbH \& Co. KG \\ Rudolf Kotschenreuther Land-Forstmaschinen \\ Schlang \& Reichert Forsttechnik / S \& R \\ TBM Tilmann Borchardt Maschinenbau \\ UTC Gesellschaft für Fahrzeug- und Maschinenbau mbH \\ Valtra Vertriebs $\mathrm{GmbH}$ \\ Wahlers Forsttechnik GmbH (Ponsse) \\ Welte Fahrzeugbau $\mathrm{GmbH}$ \\ Werner GmbH Forst- und Industrietechnik \\ WFW Waldburg Forstmaschinen Wolfegg \\ Zeppelin Baumaschinen GmbH / Bereich Forsttechnik (Caterpillar/CAT)
}

An dieser Stelle sei erwähnt, dass nicht berücksichtigte Firmen eher Nischen auf dem deutschen Markt füllen.

Der Fragenkatalog sollte u. a. Auskunft über die Vertriebszahlen - gegliedert nach Forstmaschinenkategorien - ab 1995 (oder früher) geben. Ergänzend hierzu gab es Fragen zu den Angaben der auf den einzelnen Maschinentypen eingesetzten Reifendimensionen, Einschätzungen der Unternehmen zu ihrem jeweiligen Marktanteil des zu vertreibenden Maschinentyps und der Beurteilung der Häufigkeitsverteilung von einzelnen Forstreifendimensionen auf entsprechenden Maschinentypen nach Reifenherstellern. Eine beigefügte Kurzbeschreibung des Projekts PrAllCon vervollständigte die Fragebögen abschließend und lieferte Erklärungen zu dem geplanten Forschungsvorhaben.

In einem zweiten Schritt wurden durch eine telefonische Befragung von Experten die Ergebnisse der Fragebögen ergänzt. Bei den Experten handelte es sich zum größten Teil um die Geschäftsführer der o. g. Unternehmen oder deren Vertriebsleiter. Zur Wahrung der Geschäftsinteressen wurde eine vertrauliche Behandlung der Daten zugesichert. Diese Basis, große Kooperationsbereitschaft und das hohe Interesse an dem Forschungsvorhaben veran- 
lasste die Ansprechpartner, die gewünschten Daten und Informationen (wahrscheinlich) vollständig zur Verfügung zu stellen.

Die daran anschließende methodische Vorgehensweise bestand in der Auswertung der Fragebögen und der parallel geführten Experteninterviews mit dem Ziel, einerseits die Anzahl der in deutschen Wäldern eingesetzten Maschinen zu ermitteln und andererseits eine umfassende Häufigkeitsverteilung der auf selbstfahrenden Forstmaschinen montierten Reifen zu gewinnen.

Durch die gewonnenen Daten wurde die Bearbeitung der zugrunde liegenden Fragestellung auf eine ausreichende empirische Grundlage gestellt.

\subsection{Maschinenkategorien in Deutschland}

Eine Marktanalyse von Reifen, die auf selbstfahrenden Maschinen in der deutschen Forstwirtschaft montiert sind, und deren Merkmale wurde bisher noch nicht vorgenommen.

Die gewonnenen Ergebnisse erlaubten sowohl umfassende Angaben zu den eingesetzten Maschineneinheiten gemäß der Kategorien Harvester, Forwarder, Schlepper und Landwirtschaftliche Schlepper mit Forstausrüstung als auch Aussagen zu den verwendeten Forstreifendimensionen.

Über die Entwicklung des Forstmaschinenbestandes in Deutschland geben einige Quellen Auskunft. So ist die von LEINERT (1981) durchgeführte bundesweite Vollerhebung der Regiemaschinen aus dem Jahre 1980 veraltet. Andere Veröffentlichungen beruhen auf Schätzungen, beschränken sich auf einzelne Maschinentypen oder sind landes- bzw. regionalspezifisch begrenzt. So beruht beispielsweise HAMBERGERS (2002) Darstellung der in Deutschland vorkommenden Harvester auf Schätzungen. DENNINGERS (2002) Vollerhebung (Regiemaschinen plus Maschinen forstlicher Dienstleister) des Bestandes an Harvestern ist begrenzt auf das Bundesland Niedersachsen.

Die Absicht des Kuratoriums für Waldarbeit und Forsttechnik (KWF) (NICK UND FORBRIG, 2002), den Status Quo der gesamt in Deutschland eingesetzten Maschinen wiederzugeben, war bisher nur beschränkt zu realisieren. So konnten beispielsweise Institutionen wie die Unfallversicherungsträger, die größeren Versicherungsmakler, das Kraftfahrtbundesamt oder das Bundesamt für Statistik keine präzisen Angaben zum aktuellen Forstmaschinenbestand in Deutschland machen. Die Maschinenproduzenten hingegen sahen sensible wirtschaftliche Interessen verletzt. Durch Befragung der Landesforstverwaltungen und des Deutschen Forstunternehmerverbandes (DFUV) konnte ein Teil der Forstmaschinen eruiert werden. Aber auch hier war eine Vollerhebung nicht möglich, so dass dem KWF insgesamt unvollständiges Datenmaterial vorlag und die hieraus per Hochrechnung abgeleiteten Ergebnisse letztendlich nur eine Tendenz der Entwicklung des Forstmaschinenbestandes erkennen ließen.

Die durchgeführte Marktanalyse erhebt keinen Anspruch auf Vollständigkeit, es lässt sich aber ein gegenwärtiger Bestand an Forstmaschinen ableiten, der sich nach den o. g. Maschinenkategorien in Tabelle 2 wie folgt zusammensetzt: 
Tabelle 2: Anzahl und Verteilung der in Deutschland arbeitenden Forstmaschinen (Stand: März 2002)

\begin{tabular}{|l|r|r|}
\hline \multicolumn{1}{|c|}{ Maschinenkategorien } & $\begin{array}{c}\text { Anzahl der Maschinen- } \\
\text { einheiten }\end{array}$ & $\begin{array}{c}\text { Anteil am Gesamt- } \\
\text { maschinenbestand } \\
\text { in Prozent }\end{array}$ \\
\hline Harvester & 924 & 13 \\
\hline Forwarder & 1.757 & 24 \\
\hline Schlepper & 1.351 & 19 \\
\hline $\begin{array}{l}\text { Landwirtschaftliche Schlepper } \\
\text { mit Forstausrüstung }\end{array}$ & 3.171 & 44 \\
\hline Gesamt & $\mathbf{7 . 2 0 3}$ & $\mathbf{1 0 0}$ \\
\hline
\end{tabular}

Die in Tabelle 2 bis auf die Einerziffer angegebenen Häufigkeiten sollen keine überzogene Genauigkeit suggerieren, sondern lediglich die direkten Resultate widerspiegeln. Demnach wurden im Jahr 2002924 Harvester und nahezu doppelt so viele Forwarder in Deutschland betrieben (Abb. 15). Aus dem aufgerüsteten Agrartraktorenbereich stammen die für das Rücken langer Sortimente eingesetzten Landwirtschaftlichen Schlepper mit Forstausrüstung mit 3.171 Einheiten. Sonstige Schlepper (also außerhalb der Forwarderkategorie) waren mit rund 1.351 Einheiten vertreten (EBEL UND JACKE, 2003).

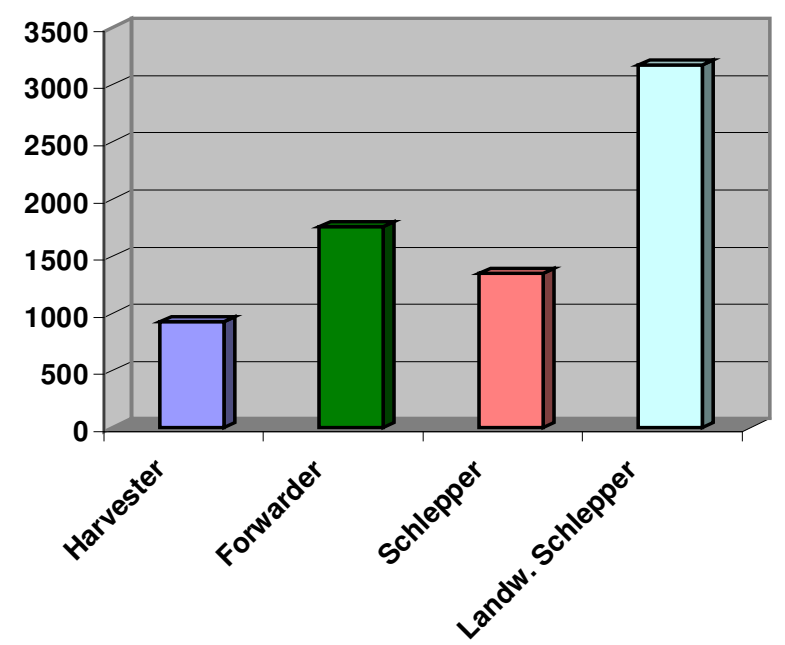

Abbildung 15: Forstmaschinenbestand in Deutschland (Stand: März 2002)

Bei einem durchschnittlichen Arbeitseinsatz eines Harvesters von 1.500 MAS p. a. (JACKE UND DREWES, 2004) und einer unterstellten mittleren Produktivität von $10 \mathrm{~m}^{3}(\mathrm{f})$ pro MAS ergibt sich für das Jahr 2002 ein (grob geschätztes) Einschlagsvolumen von knapp 14 Mio. $\mathrm{m}^{3}$. Der gesamte Holzeinschlag der Bundesrepublik Deutschland wird für das Jahr 2002 laut STATISTISCHEM JAHRBUCH (2004) mit 42 Mio. $\mathrm{m}^{3}$ angegeben. Demnach ist davon auszugehen, dass mit dem derzeitigen Maschinenbestand an Harvestern ein Drittel des jährlichen Einschlagvolumens verrichtet wird. Die Bringung des geernteten Rohholzvolumens in befahrbaren Lagen erfolgt hauptsächlich durch selbstfahrende Arbeitsmaschinen. 


\subsection{Forstreifenhersteller}

Die im Rahmen der Marktübersicht ermittelten und damit im forstlichen Bereich relevanten Forstreifen sind den nachfolgend genannten Unternehmen zuzuordnen, die an dieser Stelle kurz vorgestellt werden:

Nokian Tyres ist ein finnisches Unternehmen mit Firmensitz in der Stadt Nokia. In Deutschland werden die Reifen durch die Nokian Reifen $\mathrm{GmbH}$ in Nürnberg importiert und durch die Nokian-Generalvertretung für Schwerreifen, Bavaria Spezial Rad GmbH (B. S. R.) in FürthBislohe, vertrieben. Neben dem Vertrieb der Forstreifen fertigt die Firma B. S. R. auf Kundenwunsch auch Felgen an und kann somit vollständige Räder liefern. Mit dem Unternehmen Nokia wird in der Regel ein Industrie-Konzern mit Schwerpunkt in der Telekommunikationssparte assoziiert. Neben den anfänglichen Produkten Gummistiefel und Fahrradreifen, kamen später PKW-Reifen und Reifen für Nutzfahrzeuge hinzu. 1988 löste sich die Reifensparte aus dem Mutterkonzern und operiert heutzutage weitestgehend selbstständig. Dieser Umstand hatte gleichzeitig eine Änderung des Namens von „Nokia“ zu „Nokian“zur Folge. Zum größten Teil werden die Reifen in der Fabrik in Nokia produziert.

Trelleborg Wheel Systems ist eine Sparte des schwedischen Konzerns Trelleborg AB. Die Forstreifen werden ausschließlich in dem in der Stadt Trelleborg befindlichen Reifenwerk gefertigt. Seit 2001 gehört dem Unternehmen zudem die von Pirelli übernommene Sparte der Landwirtschaftsreifen an. Bis zum Kauf von Pirelli hatte Trelleborg Wheel Systems kaum Radialreifen im Programm, sondern traditionell fast ausschließlich Diagonalreifen und DiagonalGürtelreifen (Bias-Belted-Bauweise). Weltweit produziert Trelleborg Wheel Systems in mehreren Fabriken Land- und Forstreifen sowie Reifen für Industriefahrzeuge. Der Umstand, dass zwei Felgenwerke betrieben werden, verleiht Trelleborg Wheel Systems die Möglichkeit, gerade in der Erstausrüstung komplette Räder liefern zu können. Die Trelleborg-Vertretung in Deutschland hat ihren Sitz in Erbach im Odenwald.

Michelin, als französisches Unternehmen, ist in Deutschland durch die Michelin Reifenwerke $\mathrm{KGaA}$ in Karlsruhe vertreten, die von dort $\mathrm{u}$. a. die Vermarktung der Forstreifen steuert. Die Produktion der Reifen erfolgt indes in der spanischen Stadt Valladoid.

Firestone befindet sich in Kooperation mit Bridgestone. Die in Deutschland eingesetzten Maschinen, auf denen die Firestone-Reifen montiert sind, werden in den USA von John Deere und Caterpillar gefertigt und auch von dort mit Reifen (erst-) ausgerüstet nach Europa bzw. Deutschland geliefert. Der Vertrieb in Deutschland wird von der Bridgestone Deutschland $\mathrm{GmbH}$ in Bad Homburg übernommen.

Barum ist ein tschechischer Reifenhersteller mit Sitz in Otrokovice und gehörte seit 1993 zur Continental AG mit Sitz in Hannover. In dem tschechischen Werk werden sowohl PKW- als auch Land- und Forstwirtschaftsreifen produziert. Die ebenfalls in Tschechien hergestellten Schlepper des Unternehmens LKT werden vorwiegend mit den Barum-Reifen ausgerüstet. Die gesamte Business Unit der Landwirtschaftsreifen der Continental AG wurde im Jahr 2004 von der tschechischen Gummigesellschaft Ceská Gumárenská Spolecnost (CGS) gekauft. Dieser Betriebsübergang schloss sämtliche Geschäftprozesse von der Produktentwicklung, der Produktion bis hin zur Vermarktung mit ein, so dass u. a. der Bereich der Barum-Forst- und Landwirtschaftsreifen in Zukunft von dem Unternehmen CGS geführt werden wird. 


\subsection{Bauweisen und Dimensionen}

Die Erfassung der in der deutschen Forstwirtschaft eingesetzten Maschinen war zum einen Grundlage für die Gesamterfassung der einzelnen Reifendimensionen und zum anderen Grundlage für die auf den jeweiligen Maschinentypen montierten Forstreifen.

Zur Auswertung wurden die einen Reifen kennzeichnenden Parameter in der nachfolgenden Reihenfolge spaltenweise strukturiert und flossen in eine Kombinationsziffer ein:

\section{Reifenbreite in Zoll}

\section{Nennbreite in $\mathrm{mm}$}

3. Verhältnis Reifenhöhe zu Reifenbreite (H/B)

\section{Felgendurchmesser in Zoll}

\section{Ply-Rating-Zahl (PR-Zahl)}

\section{Last-Index / Load-Index (LI)}

\section{Reifenbauart}

$$
\begin{aligned}
& 0=\text { Diagonalreifen (inkl. bias-belted) } \\
& 1 \text { = Radialreifen (Gürtelreifen) }
\end{aligned}
$$

\section{Profilgruppen}

Um die Vielzahl der in der Forstwirtschaft verwandten Forstreifenprofile zu gliedern, erfolgte eine Orientierung anhand der am häufigsten in Erscheinung tretenden Profilklassen der Reifenhersteller Nokian und Trelleborg. Die Reifenprofile der Hersteller Michelin, Firestone und Barum sind nach Aussagen der jeweiligen Firmenvertreter mit den TRS- und ELS-Profilen von Nokian vergleichbar und wurden diesen entsprechend zugeordnet.

$\mathbf{0}=$ Breiter Antriebsreifen für Forstmaschinen, robuste Stollen, für schwierigste Verhältnisse geeignet, gute Selbstreinigungseigenschaften, robuste Reifenschulter, strapazierfähige Spezialgummimischung, wirkungsvoller Stichschutz.

Diesem Reifentyp wurden alle Profile der Unternehmen Nokian (TRS L-2, TRS LS-2, TR Forest, FS DT, AS), Michelin (FOREXBIB, XM27), Firestone (Forestry Spezial) und Barum zugeordnet.

$1=$ Rundschulterreifen mit Flachprofil für den Lastentransport mit Forstmaschinen, hohe Stundenleistung, verstärkte Seitenwände, wirkungsvoller Stichschutz.

Nur die von Nokian entwickelten Profilvarianten LS, ELS und ELS L-2 fielen unter diesen Reifentyp.

2 = Rundschulterreifen mit Flachprofil für den Lastentransport mit Forstmaschinen, hohe Stundenleistung, verstärkte Seitenwände, wirkungsvoller Stichschutz. 
3 = Breiter Antriebsreifen für Forstmaschinen, robuste Stollen, für schwierigste Verhältnisse geeignet, gute Selbstreinigungseigenschaften, robuste Reifenschulter, strapazierfähige Spezialgummimischung, wirkungsvoller Stichschutz.

Trelleborg-Profile 410, 414, 416, 425, 422

Die Ziffernfolge 9999 diente als Platzhalter und definierte allgemein einen nicht berücksichtigten Parameter.

Die Kombinationsziffer erlaubte vielfältige Gruppierungsmöglichkeiten und erleichterte eine gezielte Analyse der Daten mittels Excel und SPSS.

\section{Beispiele von Kombinationsziffern:}

Nummerncode Standardreifen: 18.4_467_100_34_14_9999_0_3

Nummerncode Niederquerschnittreifen:9999_600_55_26,5_16_9999_0_0

Die absolute Anzahl der auf den Maschinen montierten Forstreifen in Deutschland beläuft sich derweil auf 36.684. Die Verteilung auf die einzelnen Reifenhersteller ist in Tabelle 3 zu sehen.

Tabelle 3: Hersteller und Anzahl der in Deutschland eingesetzten Forstreifen (Stand: März 2002)

\begin{tabular}{|c|r|r|}
\hline Reifenhersteller & Anzahl der Forstreifen & $\begin{array}{c}\text { Anteil am Gesamtreifen- } \\
\text { bestand in Prozent }\end{array}$ \\
\hline$N$ & 21.876 & $6 C$ \\
\hline$T$ & 7.862 & 21 \\
\hline$M$ & 4.386 & 12 \\
\hline$F$ & 1.140 & 3 \\
\hline$B$ & 1.420 & 4 \\
\hline Gesamt & 36.684 & $\mathbf{1 0 0}$ \\
\hline
\end{tabular}

Zwei Hersteller halten die überwiegenden Anteile in diesem Marktsegment. Zusammen mit einem weiteren dominieren die Produkte dieser drei Anbieter auf $90 \%$ aller Maschinen in Deutschlands Wäldern diesen Markt. Reifen aus den übrigen Werken spielen eine eher untergeordnete Rolle; sie werden fast ausschließlich auf ziehenden Schleppern eingesetzt

Auf Landwirtschaftlichen Schleppern mit Forstausrüstung oder teilweiser Forstausstattung (z. B. nur Seilwinde) finden sich vereinzelt auch noch Reifen anderer namhafter Hersteller, wie Continental, Pirelli, Dunlop etc.. Diese Reifen traten aber in einem zu vernachlässigenden Umfang auf und wurden infolgedessen in der Marktanalyse nicht weiter berücksichtigt.

Die Ergebnisse gestatten also Rückschlüsse auf die Marktverteilung bzw. auf Präferenzen der Wahl von Forstreifen der Maschinenhersteller/Generalimporteure und deren Kunden. Die Wahl eines Großteils der Forstreifen wird allerdings nach Aussagen von Experten in erster Linie durch die Höhe des Preises bestimmt (FENSEL, 2002). Aspekte des Umweltschutzes bzw. des Bodenschutzes spielen eine untergeordnete Rolle.

Wie sich die Anzahl der im forstlichen Einsatz verwendeten Reifen auf die einzelnen Maschinenkategorien verteilt, ist der Tabelle 4 zu entnehmen. 
Tabelle 4: Anzahl und Verteilung der Forstreifen, gegliedert nach Maschinenkategorien (Stand: März 2002)

\begin{tabular}{|l|r|r|}
\hline \multicolumn{1}{|c|}{ Maschinenkategorien } & Anzahl der Forstreifen & $\begin{array}{c}\text { Anteil am Gesamtreifen- } \\
\text { bestand in Prozent }\end{array}$ \\
\hline Harvester & 5.192 & 14 \\
\hline Forwarder & 13.370 & 36 \\
\hline Schlepper & 5.438 & 15 \\
\hline $\begin{array}{l}\text { Landwirtschaftliche Schlepper } \\
\text { mit Spezialausrüstung }\end{array}$ & 12.684 & 35 \\
\hline Gesamt & $\mathbf{3 6 . 6 8 4}$ & $\mathbf{1 0 0}$ \\
\hline
\end{tabular}

Demnach steht die Reifenhäufigkeit, die den aufgerüsteten Landwirtschaftlichen Schleppern zuzuordnen ist (35\%), nahezu gleichrangig der der Forwarderreifen gegenüber (36\%). Bei einer (hier mit Vorsicht möglichen) Hochrechnung der Maschinenzahlen (Tab. 2) ist zu beachten, dass die aufgerüsteten Agrarschlepper stets mit vier, die Forwarder hingegen mit sechs oder gar acht Rädern bestückt sind. Eine ähnliche Rechnung gilt für die Gegenüberstellung von Harvester- und sonstiger Spezialschlepperbereifung: Skidder mit jeder möglichen Beizugs-, Transport- und Polterhilfe weisen i. d. R. nicht mehr als vier, die Harvester hingegen häufig sechs Räder auf.

Eine detaillierte Darstellung der Reifendimensionen, geordnet nach Standard- und Niederquerschnittreifen, zeigen die Tabellen 5 und 6. Die Grundlage für diese Erhebung bildete die oben genannte Kombinationsziffer.

Tabelle 5: In der forstlichen Praxis auf selbstfahrenden Maschinen eingesetzte Standardreifen (Stand: März 2002)

\begin{tabular}{|l|r|r|}
\hline Kombinationsziffer/ Nummerncode & Häufigkeit & Prozent \\
\hline 14,9_378_100_24,0_12_9999_0_3 & 132 & 1,2 \\
14,9_378_100_24,0_14_9999_0_0 & 1378 & 12,7 \\
14,9_378_100_24,0_9999_126_1_0 & 830 & 7,6 \\
14,9_378_100_28,0_12_9999_0_3 & 99 &, 9 \\
14,9_378_100_28,0_14_9999_0_0 & 245 & 2,3 \\
16,9_429_100_28,0_14_9999_0_0 & 216 & 2,0 \\
16,9_429_100_30,0_14_9999_0_9999 & 1870 & 17,2 \\
16,9_429_100_30,0_9999_137_1_0 & 450 & 4,1 \\
18,4_467_100_30,0_14_9999_0_3 & 30 &, 3 \\
18,4_467_100_30,0_14_9999_0_9999 & 450 & 4,1 \\
18,4_467_100_30,0_16_9999_0_0 & 68 &, 6 \\
18,4_467_100_30,0_9999_142_1_0 & 450 & 4,1 \\
18,4_467_100_34,0_14_9999_0_0 & 1578 & 14,5 \\
18,4_467_100_34,0_14_9999_0_3 & 132 & 1,2 \\
18,4_467_100_34,0_9999_144_1_0 & 830 & 7,6 \\
18,4_467_100_38,0_14_9999_0_0 & 245 & 2,3 \\
18,4_467_100_38,0_14_9999_0_3 & 99 &, 9 \\
20,8_528_100_38,0_20_9999_0_0 & 216 & 2,0 \\
23,1_587_100_26,0_14_9999_0_0 & 544 & 5,0 \\
23,1_587_100_26,0_16_9999_0_0 & 285 & 2,6 \\
24,56622_100_32,0_16_9999_0_0 & 104 & 1,0 \\
28L_714_100_26,0_14_9999_0_0 & 492 & 4,5 \\
28L_714_100_26,0_16_9999_0_0 & 120 & 1,1 \\
Gesamt & 10863 & 100,0 \\
\hline
\end{tabular}


Die Standardreifen werden hauptsächlich auf den Landwirtschaftlichen Schleppern mit Forstausrüstung und auf Schleppern verwendet. Vor allem die Dimensionen 14.9, 16.9 und 18.4 treten besonders hervor (Tab. 5).

Die speziell für die forstliche Praxis entwickelten Niederquerschnittreifen bilden einen Schwerpunkt im Bereich der Nennbreiten $600 \mathrm{~mm}$ und $700 \mathrm{~mm}$ (Tab. 6). Normalhin werden Harvester und Forwarder mit diesen Reifen ausgerüstet. Eine ausführliche Dokumentation der einzelnen Reifendimensionen auf den Radachsen der jeweiligen Maschinentypen ist im nachfolgenden Kapitel 3.5 zu finden.

Tabelle 6: In der forstlichen Praxis auf selbstfahrenden Maschinen eingesetzte Niederquerschnittreifen (Stand: März 2002)

\begin{tabular}{|c|c|c|}
\hline Kombinationsziffer / Nummerncode & Häufigkeit & Prozent \\
\hline 9999_400_55_22,5_8_9999_0_1 & 8 &, 0 \\
\hline 9999_500_45_22,5_12_9999_0_3 & 1400 & 5,4 \\
\hline 9999_500_60_22,5_16_9999_0_1 & 48 & ,2 \\
\hline 9999_500_60_22,5_16_9999_0_3 & 12 &, 0 \\
\hline 9999_500_60_26,5_12_9999_0_0 & 217 & ,8 \\
\hline 9999_500_60_26,5_12_9999_0_3 & 4 & 0 \\
\hline 9999_500_60_26,5_14_9999_0_3 & 79 & ,3 \\
\hline 9999_500_65_24,0_9999_142_1_0 & 130 &, 5 \\
\hline 9999_500_65_26,5_14_9999_0_3 & 76 & , 3 \\
\hline 9999_500_65_28,0_9999_144_1_0 & 1059 & 4,1 \\
\hline 9999_500_75_24,0_9999_154_1_0 & 458 & 1,8 \\
\hline 9999_520_75_30,0_9999_159_1_0 & 180 &, 7 \\
\hline 9999_540_60_26,5_9999_145_1_0 & 400 & 1,5 \\
\hline 9999_540_65_28,0_9999_149_1_0 & 375 & 1,5 \\
\hline 9999_540_75_38,0_9999_165_1_0 & 288 & 1,1 \\
\hline 9999_550_45_22,5_12_9999_0_1 & 168 &, 7 \\
\hline 9999_600_50_22,5_12_9999_0_0 & 136 &, 5 \\
\hline 9999_600_50_22,5_12_9999_0_1 & 216 & ,8 \\
\hline 9999_600_50_22,5_12_9999_0_2 & 1928 & 7,5 \\
\hline 9999_600_50_22,5_16_9999_0_0 & 2579 & 10,0 \\
\hline 9999_600_50_22,5_16_9999_0_1 & 80 & ,3 \\
\hline 9999_600_50_22,5_16_9999_0_2 & 3 & 0 \\
\hline 9999_600_55_22,5_9999_162_0_1 & 96 & ,4 \\
\hline 9999_600_55_26,5_12_9999_0_0 & 74 & , 3 \\
\hline 9999_600_55_26,5_14_9999_0_0 & 10 & 0 \\
\hline 9999_600_55_26,5_14_9999_0_3 & 1182 & 4,6 \\
\hline 9999_600_55_26,5_16_9999_0_0 & 3117 & 12,1 \\
\hline 9999_600_55_26,5_16_9999_0_1 & 980 & 3,8 \\
\hline 9999_600_55_26,5_16_9999_0_2 & 970 & 3,8 \\
\hline 9999_600_55_26,5_9999_172_1_1 & 32 &, 1 \\
\hline 9999_600_60_30,5_14_9999_0_3 & 1092 & 4,2 \\
\hline 9999_600_65_34,0_14_9999_0_0 & 1164 & 4,5 \\
\hline 9999_600_65_34,0_14_9999_0_2 & 116 & ,4 \\
\hline 9999_600_65_34,0_14_9999_0_3 & 110 & ,4 \\
\hline 9999_600_65_34,0_9999_157_1_0 & 16 & 1 \\
\hline 9999_600_65_38,0_9999_159_1_0 & 1501 & 5,8 \\
\hline 9999_650_65_26,5_20_9999_0_1 & 37 &, 1 \\
\hline 9999_650_65_38,0_9999_164_1_0 & 367 & 1,4 \\
\hline 9999_700_40_22,5_12_9999_0_2 & 8 &, 0 \\
\hline 9999_700_40_22,5_16_9999_0_2 & 67 & ,3 \\
\hline 9999_700_45_22,5_16_9999_0_0 & 1565 & 6,1 \\
\hline 9999_700_50_26,5_16_9999_0_0 & 1520 & 5,9 \\
\hline 9999_700_50_26,5_16_9999_0_1 & 624 & 2,4 \\
\hline 9999_700_50_26,5_16_9999_0_2 & 157 & ,6 \\
\hline 9999_700_55_34,0_14_9999_0_0 & 992 & 3,8 \\
\hline 9999_700_55_34,0_14_9999_0_2 & 126 &, 5 \\
\hline 9999_700_70_34,0_16_9999_0_0 & 14 &, 1 \\
\hline 9999_800_40_26,5_20_9999_0_2 & 40 & , 2 \\
\hline Gesamt $--1---$ & 25821 & 100,0 \\
\hline
\end{tabular}


Als weiteres Ergebnis ist zu konstatieren, dass rund $70 \%$ der im Wald gefahrenen Reifen nach dem Niederquerschnittprinzip konstruiert sind, die verbleibenden $30 \%$ nach dem Standardkonzept.

In der globalen Aufteilung sind $80 \%$ aller Reifen konventionell diagonal gebaut, $20 \%$ sind in der Basiskarkasse radial strukturiert. Das Verhältnis von Diagonal- zu Radialreifen beträgt somit $4: 1$. Die Ergebnisse sind im Anhang 2 in den Tabellen 2.1 und 2.2 dargestellt. Den Abbildungen 16 und 17 ist zu entnehmen, dass sowohl bei den Niederquerschnitt- als auch bei den Standardreifen die Diagonalbauweise deutlich dominiert.

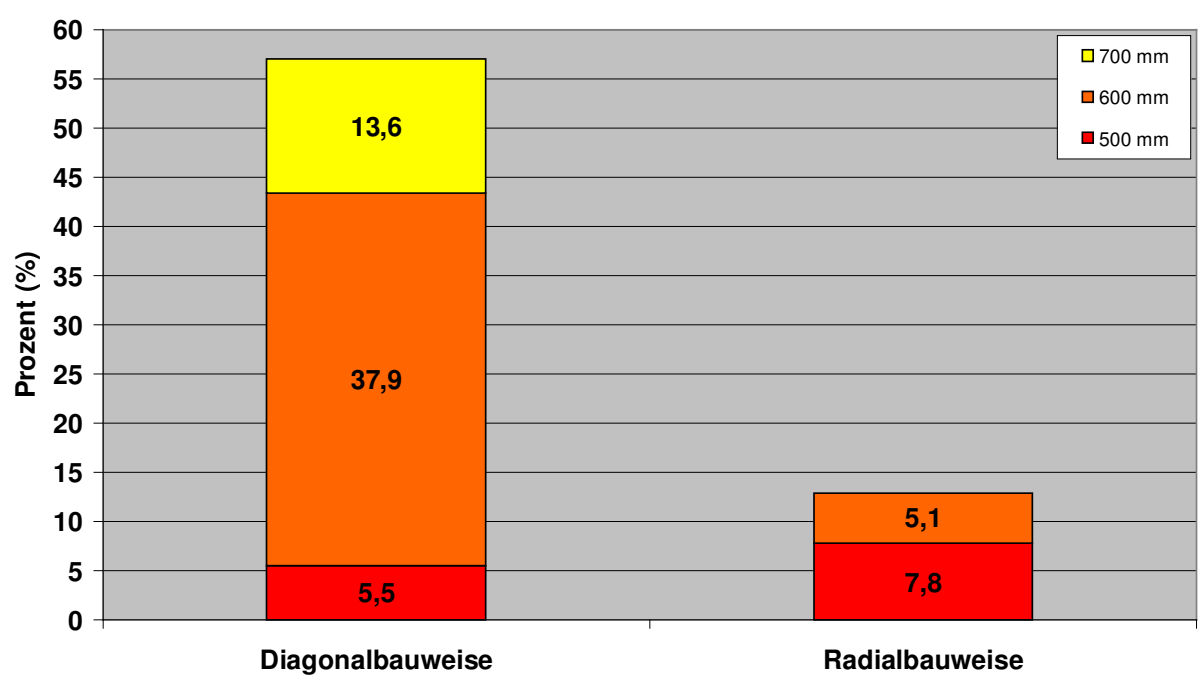

Abbildung 16: Niederquerschnittreifen in Diagonal- und Radialbauweise, nach Nennbreiten in Millimeter (auf eine Darstellung der 400er und 800er Bereifung wurde in Anbetracht der marginalen Anteile verzichtet (Tab. 9))

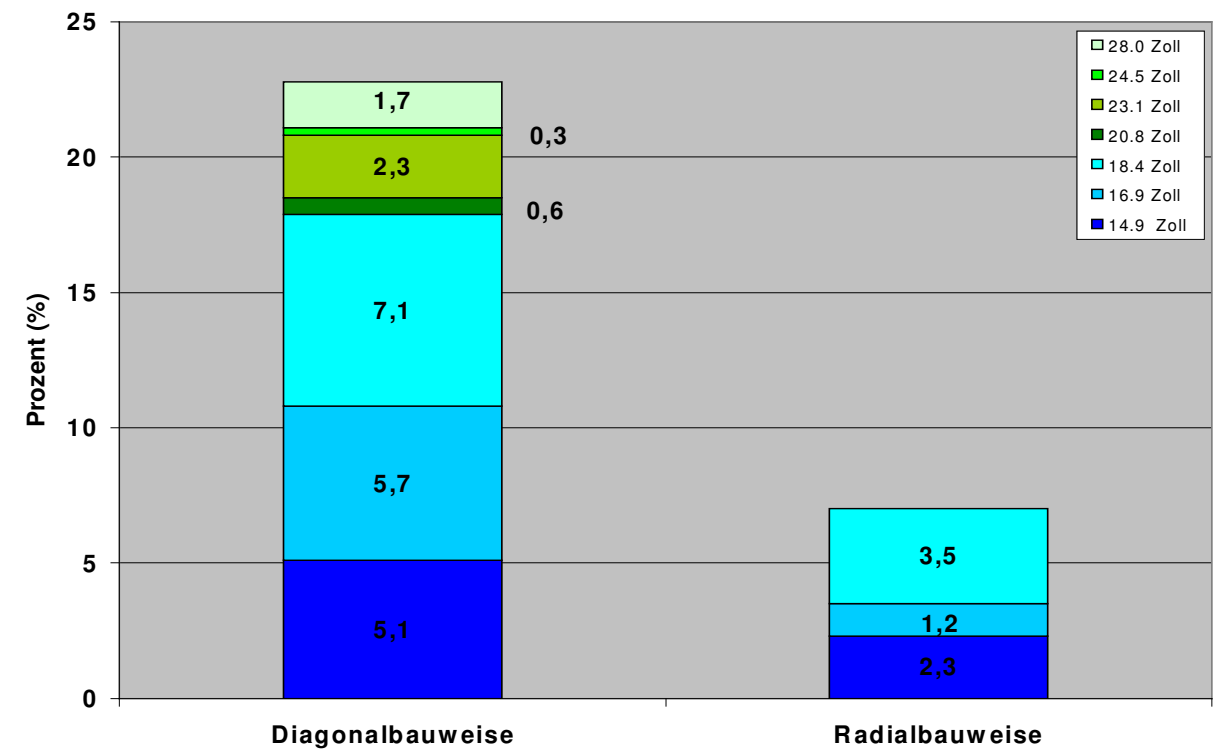

Abbildung 17: Standardreifen in Diagonal- und Radialbauweise, gegliedert nach Nennbreiten in Zoll 


\subsection{Verwendung von Forstreifen}

Eine Disposition nach Nennbreiten sollte klären, auf welchen Fahrwerken die einzelnen Reifen montiert werden. Dabei erschien es sinnvoll, die einzelnen Maschinenkategorien näher zu untersuchen. Infolgedessen wurde eine Aufschlüsselung in 4-, 6- und 8-Rad-Maschinen innerhalb der jeweiligen Maschinenkategorie vorgenommen (Tab. 7 und 8).

Tabelle 7: Anzahl der Forstreifen auf Harvestern und Forwardern mit unterschiedlichen Fahrwerken (Stand: März 2002)

\begin{tabular}{|l|r|r|r|r|}
\hline Maschinentypen & \multicolumn{1}{|c|}{ Harvester } & $\begin{array}{c}\text { Anzahl } \\
\text { Forstreifen }\end{array}$ & Forwarder & \multicolumn{1}{c|}{$\begin{array}{c}\text { Anzahl } \\
\text { Forstreifen }\end{array}$} \\
\hline 4-Rad-Maschinen & 235 & 940 & 1 & 4 \\
\hline 6-Rad-Maschinen & 630 & 3.780 & 341 & 2.046 \\
\hline 8-Rad-Maschinen & 59 & 472 & 1.415 & 11.320 \\
\hline
\end{tabular}

Tabelle 8: Anzahl der Forstreifen auf Schleppern und Landwirtschaftlichen Schleppern mit Forstausrüstung mit unterschiedlichen Fahrwerken (Stand: März 2002)

\begin{tabular}{|l|r|r|rc|c|}
\hline Maschinentypen & Schlepper & $\begin{array}{c}\text { Anzahl } \\
\text { Forstreifen }\end{array}$ & $\begin{array}{c}\text { Landwirtschaftliche } \\
\text { Schlepper mit Forstaus- } \\
\text { rüstung }\end{array}$ & $\begin{array}{c}\text { Anzahl } \\
\text { Forstreifen }\end{array}$ \\
\hline 4-Rad-Maschinen & 1.334 & 5.336 & & 3.171 & 12.684 \\
\hline 6-Rad-Maschinen & 17 & 102 & -- & -- \\
\hline 8-Rad-Maschinen & \multicolumn{5}{|c}{--} \\
\hline
\end{tabular}

Eine weitere Differenzierung erfolgte hinsichtlich der auf den Maschinen verwendeten Forstreifen. So wurde das Reifenkollektiv in diagonal- und radial gebaute Niederquerschnittreifen und Standardreifen gruppiert. Eine ausführliche Übersicht vermitteln die Tabellen 9 bis 12 .

Innerhalb der diagonal gebauten Niederquerschnittreifen rangiert die Nennbreite $600 \mathrm{~mm}$ mit $38 \%$ an erster Stelle (Tab. 9). Hier finden sich z. B. nahezu alle Forwarderreifen und die auf Bogie-Achsen montierten Reifen sechsrädriger Harvester. Ähnlich verhält es sich mit den 700er Bereifungen. Am häufigsten werden die Größen: 600/55-26.5, 600/60-30.5, 600/65-34, 700/45-22.5 und 700/50-26.5 sowohl auf 4-Rad- als auch auf 8-Rad-Maschinen montiert.

Ein ebenfalls sehr hoher Anteil mit 3,8\% innerhalb der Klasse der 500er Bereifung, ist auf dem von Timberjack produzierten Forwarder „Mini Brunett“ (seinerzeit noch ca. 350 Exemplare in der forstlichen Praxis) eingesetzt. Mit einem prozentualen Anteil von 0,1 am Gesamtkollektiv sind die 800er Dimensionen besonders schwach vertreten.

Die radial geschlagenen Niederquerschnittreifen gehören vollzählig zu Landwirtschaftlichen Schleppern mit Forstausrüstung (Tab. 10): die Nennbreiten von 500 mm bis 540 mm werden zu $90 \%$ auf der Vorderachse, Nennbreiten ab 600 mm auf der (großrädrigen) Hinterachse montiert.

Standardreifen in diagonaler Bauweise haben ihre Domäne auf Landwirtschaftlichen Schleppern mit Forstausrüstung (Tab. 11). Bei diesen Bereifungen handelt es sich einerseits um konventionelle Ackerschlepperreifen mit hoher Karkassenfestigkeit (PR-Zahlen von 12 und darüber), andererseits umfasst die Produktpalette einiger Reifenhersteller besonders für die Forstwirtschaft abgestimmte - nach Nomenklatur der Hersteller - „Agroforest oder Forstreifen 
für Landwirtschaftstraktoren“. Darüber hinaus finden sich auf Schleppern ebenfalls speziell für die Forstwirtschaft konzipierte Standardreifen, wie z. B. die Dimensionen 23.1-26 oder 28 L-26.

Auch die Standardreifen in radialer Bauweise gehören vorwiegend zur Maschinenkategorie Aufgerüsteter Landwirtschaftstraktoren (Tab. 12). Rund $80 \%$ aller Standardreifen werden auf Landwirtschaftlichen Schleppern mit Forstausrüstung gefahren, die restlichen $20 \%$ auf Schleppern.

Niederquerschnittreifen in diagonaler und radialer Bauweise:

Tabelle 9: Verwendung von Niederquerschnittreifen in Diagonalbauweise

\begin{tabular}{|c|c|c|}
\hline $\begin{array}{l}\text { Nennbreite [mm] / } \\
\text { H/B-Verhältnis [\%] / } \\
\text { Felgendurch- } \\
\text { messer [Zoll] }\end{array}$ & Verwendung & $\begin{array}{l}\text { Gesamt- } \\
\text { häufigkeit } \\
\text { in Prozent }\end{array}$ \\
\hline \multirow[t]{2}{*}{$400 / 55-22.5$} & Vorderachsen und Hinterachsen von Forwardern (8-Rad-Ma.) (100 \%) & 0,02 \\
\hline & & 0,02 \\
\hline $500 / 45-22.5$ & $\begin{array}{l}\text { Vorderachsen und Hinterachsen von Forwardern } \\
(100 \% \rightarrow \text { Mini Brunett) }\end{array}$ & 3,80 \\
\hline $500 / 60-22.5$ & $\begin{array}{l}\text { Vorderachsen und Hinterachsen von Forwardern (80 \%) } \\
\text { Vorderachsen von Harvestern (20\%) }\end{array}$ & 0,20 \\
\hline $500 / 60-26.5$ & $\begin{array}{l}\text { Vorderachsen von Landwirtschaftlichen Schleppern mit Forstausrüstung } \\
(\sim 66 \%) \\
\text { Vorderachsen und Hinderachsen von Harvestern, Forwardern und } \\
\text { Schleppern }(\sim 33 \%)\end{array}$ & 1,00 \\
\hline \multirow[t]{2}{*}{$550 / 45-22.5$} & $\begin{array}{l}\text { Vorderachsen und Hinterachsen von Forwardern (8-Rad-Ma.) } \\
(100 \%)\end{array}$ & 0,50 \\
\hline & & 5,50 \\
\hline $600 / 50-22.5$ & $\begin{array}{l}\text { Vorderachsen (6-Rad-Ma.) + Vorderachsen und Hinterachsen (8-Rad- } \\
\text { Ma.) von Forwardern ( 95\%) } \\
\text { Vorderachsen von Harvestern ( } 5 \%)\end{array}$ & 13,50 \\
\hline $600 / 55-26.5$ & $\begin{array}{l}\text { Vorderachsen von Harvestern ( 21 \%) } \\
\text { Vorderachsen }(6-\text { Rad-Ma.) + Vorderachsen und Hinterachsen (8-Rad- } \\
\text { Ma.) von Forwardern }(\sim 59 \%) \\
\text { Vorderachsen und Hinderachsen von Schleppern }(\sim 19 \%)\end{array}$ & 17,60 \\
\hline $600 / 60-30.5$ & $\begin{array}{l}\text { Hinterachsen von Landwirtschaftlichen Schleppern mit Forstausrüstung } \\
\text { + Vorderachsen und Hinterachsen von Schleppern }(\sim 87 \%) / \\
\text { Hinterachsen (6-Rad-Ma.) + Vorderachsen und Hinderachsen von } \\
\text { Harvestern (8-Rad-Ma.) ( 13\%) }\end{array}$ & 3,00 \\
\hline $600 / 65-34$ & $\begin{array}{l}\text { Hinterachsen (6-Rad-Ma.) + Vorderachsen und Hinterachsen von Har- } \\
\text { vestern (8-Rad-Ma.) ( 50 \%) } \\
\text { Vorderachsen von Forwardern ( 35\%) } \\
\text { Vorderachsen und Hinterachsen von Schleppern ( 15\%) }\end{array}$ & 3,80 \\
\hline \multirow[t]{2}{*}{$650 / 65-26.5$} & $\begin{array}{l}\text { Vorderachsen (6 Rad-Ma.) + Vorderachsen und Hinterachsen von } \\
\text { Harvestern (8-Rad-Ma.) (100 \%) }\end{array}$ & 0,10 \\
\hline & & 38,0 \\
\hline $700 / 45-22.5$ & $\begin{array}{l}\text { Vorderachsen und Hinterachsen von Forwardern ( 8-Rad-Ma.) ( 71 \%) } \\
\text { Vorderachsen (6 Rad-Ma.) + Vorderachsen und Hinterachsen von } \\
\text { Harvestern ( 8-Rad-Ma.) ( 29\%) }\end{array}$ & 4,20 \\
\hline $700 / 50-26.5$ & $\begin{array}{l}\text { Hinterachsen (6-Rad-Ma.) + Vorderachsen und Hinterachsen (8-Rad- } \\
\text { Ma.) von Forwardern }(\sim 53 \%) \\
\text { Vorderachsen von Harvestern ( } 43 \%) \\
\text { Vorderachsen und Hinterachsen von Schleppern ( 4\%) }\end{array}$ & 6,30 \\
\hline 700/55-34 & $\begin{array}{l}\text { Hinterachsen + Vorderachsen und Hinterachsen (4-Rad-Ma.) von Har- } \\
\text { vestern ( 98\%) } \\
\text { Vorderachsen von Forwardern (6-Rad-Ma.) ( 2\%) }\end{array}$ & 3,10 \\
\hline & & 13,60 \\
\hline \multirow[t]{2}{*}{$800 / 40-26.5$} & Vorderachsen und Hinterachsen von Forwardern ( 8-Rad-Ma.) (100 \%) & 0,10 \\
\hline & & 0,10 \\
\hline
\end{tabular}


Tabelle 10: Verwendung von Niederquerschnittreifen in Radialbauweise

\begin{tabular}{|c|l|r|}
\hline $\begin{array}{c}\text { Nennbreite in [mm] / } \\
\text { H/B-Verhältnis [\%] / } \\
\text { Felgendurch- } \\
\text { messer [Zoll] }\end{array}$ & \multicolumn{1}{|c|}{ Verwendung } & $\begin{array}{r}\text { Gesamt- } \\
\text { häufigkeit } \\
\text { in Prozent }\end{array}$ \\
\hline $500 / 65$ R 24 & Vorderachsen und Hinterachsen von Schleppern ( 100 \%) & 0,40 \\
\hline $500 / 65$ R 28 & $\begin{array}{l}\text { Vorderachsen von Landwirtschaftlichen Schleppern mit Forstausrüstung } \\
(100 \%)\end{array}$ & 2,90 \\
\hline $500 / 75$ R 24 & $\begin{array}{l}\text { Vorderachsen von Landwirtschaftlichen Schleppern mit Forstausrüstung } \\
(100 \%)\end{array}$ & 1,20 \\
\hline $520 / 75$ R 30 & Vorderachsen und Hinderachsen von Schleppern (100 \%) & 0,50 \\
\hline $540 / 60$ R 26.5 & $\begin{array}{l}\text { Vorderachsen von Landwirtschaftlichen Schleppern mit Forstausrüstung } \\
(100 \%)\end{array}$ & 2,10 \\
\hline $540 / 65$ R 28 & $\begin{array}{l}\text { Hinterachsen von Landwirtschaftlichen Schleppern mit Forstausrüstung } \\
(100 \%)\end{array}$ & 0,80 \\
\hline $540 / 75$ R 38 & $\begin{array}{l}\text { Hinterachsen von Landwirtschaftlichen Schleppern mit Forstausrüstung } \\
(100 \%)\end{array}$ & $\mathbf{7 , 9 0}$ \\
\hline & $\begin{array}{l}\text { Hinterachsen von Landwirtschaftlichen Schleppern mit Forstausrüstung } \\
(100 \%)\end{array}$ & 4,10 \\
\hline $600 / 65$ R 38 & & 1,00 \\
\hline $650 / 65$ R 38 & & $\mathbf{5 , 1 0}$ \\
\hline
\end{tabular}

\section{Standardreifen in diagonaler und radialer Bauweise:}

Tabelle 11: Verwendung von Standardreifen in Diagonalbauweise

\begin{tabular}{|c|c|c|}
\hline $\begin{array}{l}\text { Reifenbreite [Zoll] / } \\
\text { Felgendurch- } \\
\text { messer [Zoll] }\end{array}$ & Verwendung & $\begin{array}{l}\text { Gesamt- } \\
\text { häufigkeit } \\
\text { in Prozent }\end{array}$ \\
\hline $\begin{array}{l}14.9-24 \\
14.9-28\end{array}$ & $\begin{array}{l}\text { Vorderachsen von Landwirtschaftlichen Schleppern mit Forstausrüstung } \\
(\sim 100 \%)\end{array}$ & 5,10 \\
\hline $16.9-28$ & $\begin{array}{l}\text { Vorderachsen von Landwirtschaftlichen Schleppern mit Forstausrüstung } \\
(100 \% \rightarrow \text { Valtra) }\end{array}$ & 0,60 \\
\hline $16.9-30$ & $\begin{array}{l}\text { Vorderachsen und Hinterachsen von Schleppern }(\sim 60 \%) \\
\text { Vorderachsen und Hinterachsen von Landwirtschaftlichen Schleppern } \\
\text { mit Forstausrüstung ( } 40 \% \rightarrow \text { MB-Trac) }\end{array}$ & 5,10 \\
\hline $18.4-30$ & $\begin{array}{l}\text { Vorderachsen und Hinterachsen von Landwirtschaftlichen Schleppern } \\
\text { mit Forstausrüstung }(\sim 82 \% \rightarrow \text { MB-Trac) } \\
\text { Hinterachsen von Forwardern (6-Rad-Ma.) + Vorderachsen und Hinter- } \\
\text { achsen von Harvestern (8-Rad-Ma.) }(\sim 18 \%)\end{array}$ & 1,50 \\
\hline $\begin{array}{l}18.4-34 \\
18.4-38\end{array}$ & $\begin{array}{l}\text { Hinterachsen von Landwirtschaftlichen Schleppern mit Forstausrüstung } \\
(100 \%)\end{array}$ & 5,60 \\
\hline 20.8-38 & $\begin{array}{l}\text { Hinterachsen von Landwirtschaftlichen Schleppern mit Forstausrüstung } \\
(100 \%)\end{array}$ & 0,60 \\
\hline $23.1-26$ & $\begin{array}{l}\text { Vorderachsen und Hinterachsen von Schleppern ( } 80 \%) \\
\text { Vorderachsen von Forwardern (6-Rad-Ma.) }(\sim 20 \%)\end{array}$ & 2,30 \\
\hline $24.5-32$ & Vorderachsen und Hinterachsen von Schleppern (100 \%) & 0,30 \\
\hline $28 L-26$ & Vorderachsen und Hinterachsen von Schleppern ( 100\%) & 1,70 \\
\hline & & 22,80 \\
\hline
\end{tabular}

Tabelle 12: Verwendung von Standardreifen in Radialbauweise

\begin{tabular}{|c|c|c|}
\hline $\begin{array}{l}\text { Reifenbreite [Zoll] / } \\
\text { Felgendurch- } \\
\text { messer [Zoll] }\end{array}$ & Verwendung & $\begin{array}{l}\text { Gesamt- } \\
\text { häufigkeit } \\
\text { in Prozent }\end{array}$ \\
\hline 14.9 R 24 & $\begin{array}{l}\text { Vorderachsen von Landwirtschaftlichen Schleppern mit Forstausrüstung } \\
(100 \%)\end{array}$ & 2,30 \\
\hline 16.9 R 30 & $\begin{array}{l}\text { Vorderachsen und Hinderachsen von Landwirtschaftlichen Schleppern } \\
\text { mit Forstausrüstung (100\% } \rightarrow \text { MB-Trac) }\end{array}$ & 1,20 \\
\hline $\begin{array}{l}18.4 \text { R } 30 \\
18.4 \text { R } 34\end{array}$ & $\begin{array}{l}\text { Vorderachsen und Hinderachsen von Landwirtschaftlichen Schleppern } \\
\text { mit Forstausrüstung }(\sim 35 \% \rightarrow \text { MB-Trac }) \\
\text { Hinderachsen von Landwirtschaftlichen Schleppern mit Forstausrüstung } \\
(\sim 65 \%)\end{array}$ & 3,50 \\
\hline & & 7,00 \\
\hline
\end{tabular}


Generell wurde festgestellt, dass auf Harvestern und Forwardern ausschließlich Niederquerschnittreifen in Diagonalbauweise montiert werden; auf Skiddern und Landwirtschaftlichen Schleppern mit Forstausrüstung kommen dagegen sowohl Diagonal- als auch Radialreifen zum Einsatz. Innerhalb der diagonal gebauten Niederquerschnittreifen rangieren die Nennbreiten $600 \mathrm{~mm}$ und $700 \mathrm{~mm}$ an erster Stelle. Sie nehmen mit fast $52 \%$ eine exponierte Stellung unter den in der Forstwirtschaft verwendeten Reifen ein. Nennbreiten mit 800 mm sind derzeit außerordentlich schwach vertreten.

Insgesamt ergibt sich ein Verhältnis von diagonal zu radial gebauten Niederquerschnittreifen von $4: 1$ - das Verhältnis der Standarddiagonalreifen zu Standardradialreifen beträgt $3: 1$.

\subsection{Nutzungsdauer}

Forstreifen zeigen heute eine erstaunliche „Lebenserwartung“, denn das im ausgesprochenen Straßenbetrieb gefürchtete „Altern“ der Gummimischungen, die (nicht durch den Einsatz bedingten) materialschwächenden Faktoren spielen im forstlichen Einsatzbereich nicht die Rolle für die Begrenzung der Nutzungszeit. Letztere wird geprägt von eher mechanischen Beanspruchungen, die zu Karkassenverletzungen, Profilabbrüchen oder auch schlicht Abrieb führen. Dies bedeutet, dass Reifen, die auf Forstmaschinen mit relativ geringen Fahrdistanzen (Harvester) montiert werden, maximale Nutzungsdauern erreichen. Auch Forwarder werden auf größerer Distanz per Tieflader umgesetzt, aber allein der mit innen bewältigte Rückeprozess bedingt deutlich größere Fahrdistanzen innerhalb der Maschinennutzungsdauer, so dass während dieses Zeitraumes ein Wechsel der Erstbereifung wahrscheinlich erscheint.

Die noch größeren Fahrdistanzen der sonstigen Schlepper und insbesondere der verbreiteten, forstlich aufgerüsteten landwirtschaftlichen Schlepper, die auch weitere Umsetzungsstrecken selbstfahrend bewältigen müssen (vermehrtes Befahren von befestigten Wegen und Straßen), lässt auf eine Zweit- bzw. Drittbereifung während der Maschinennutzungsdauer schließen. Dies ist deutlich bei einem Vergleich der Gesamtnutzungsdauer der Maschinen und der maximalen Nutzungsdauer der Forstreifen bei den Schleppern und Landwirtschaftlichen Schleppern mit Forstausrüstung zu erkennen.

Über die Beanspruchung der Reifen und deren maximale Nutzungsdauer in Betriebsstunden $(B S)^{5}$, in Abhängigkeit von der Gesamtnutzungsdauer des jeweiligen Maschinentyps, gibt Tabelle 13 Auskunft.

\footnotetext{
${ }^{5}$ Betriebsstunde (BS): Maschine ist in Betrieb, Motor (falls vorhanden) läuft; der Betriebszustand ist über eine Getriebewelle (veraltet) oder über einen Lichtmaschinenzusatz, neuerdings auch über einen CAN-BUS kontrollierbar.
} 
Tabelle 13: Gesamtnutzungsdauer der Maschinen und maximale Nutzungsdauer der darauf montierten Forstreifen

\begin{tabular}{|l|r|r|}
\hline \multicolumn{1}{|c|}{ Maschinenkategorien } & $\begin{array}{c}\text { Gesamtnutzungsdauer } \\
\text { der Maschinen in } \mathbf{B S}^{6}\end{array}$ & $\begin{array}{c}\text { Maximale Nutzungsdauer } \\
\text { der Forstreifen in } \mathbf{B S}^{\mathbf{7}}\end{array}$ \\
\hline Harvester & $14.000-15.000$ & $10.000-15.000$ \\
\hline Forwarder & $17.000-18.000$ & $8.000-12.000$ \\
\hline Schlepper & 19.000 & 8.000 \\
\hline $\begin{array}{l}\text { Landwirtschaftliche Schlepper } \\
\text { mit Forstausrüstung }\end{array}$ & 11.000 & $4.000-7.000$ \\
\hline
\end{tabular}

\subsection{Konsequenzen für die zu bemessenden Forstreifen}

Die bis hier ausgewerteten Ergebnisse der Forstreifen-Marktanalyse lieferten in ihren Details die Grundlage für den Entscheidungsprozess, welche Reifen innerhalb der Messungen des PrAllCon-Projekts berücksichtigt werden müssen. Hierfür wurden die Forstreifen nach Reifenherstellern selektiert, anhand der einen Reifen beschreibenden Merkmale (Reifenparameter) geordnet und eine Gewichtung nach Häufigkeitsverteilung vorgenommen.

Die vorwiegend retrospektiv ausgerichteten Erhebungen dieser Art würdigten nur unzureichend Reifenneuentwicklungen, die demzufolge (noch) relativ selten auf den Maschinen montiert sind - beispielsweise Profile, deren Stollen zu den Reifenschultern betont stark ausgelegt sind (Forest King F von Nokian oder die Trelleborg-Profile 422 und 428). Auch solche zukunftsträchtige Reifen werden hinsichtlich ihrer Kontaktflächendruckverteilung untersucht.

Zur Senkung des Schwerpunktes bei gleichzeitiger Verbesserung der Stabilität (z. B. bei groBen Auslegern) werden vorwiegend Reifen auf den Vorderachsen von Harvestern häufig mit Wasser befüllt (s. Kap. 1.4). Das Gewicht des Wassers stellt eine Erhöhung der Radlast und somit eine weitere Belastung des Bodens in Form einer möglichen Veränderung der Kontaktflächendruckverteilung unterhalb des Reifens dar, die es ebenfalls näher zu untersuchen gilt.

\subsection{Herkunft des Datenmaterials/Forstreifenkollektivs}

Die oben genannten Reifenhersteller erklärten sich bereit, die im Rahmen der Marktanalyse ermittelten Forstreifen als unentgeltliche Sachmittelleistung (Gestellung von kompletten Rädern inklusive An- und Abtransport) zur Verfügung zu stellen.

Dabei wurden die Reifen i. d. R. in Gesamtpaketen oder in mehreren Lieferungen (z. B. Trelleborg, Abb. 18) ohne Zeitbegrenzung bereitgestellt. Die Nokian-Reifen bildeten hierbei eine Ausnahme. Da die Firma B. S. R. ausschließlich als Händler auftritt und komplette Räder nur auf Kundenwunsch fertigt, bestanden in diesem Fall Restriktionen hinsichtlich der Anzahl der Räder (5 - 7 Reifen pro Lieferung) und befristete Zeitlimits während der Bemessung.

Insgesamt konnte während des Projekts auf ein Reifenkollektiv zurückgegriffen werden, dass 70 Forstreifen in allen derzeit gebräuchlichen Dimensionen umfasst (s. hierzu Anhang 3).

\footnotetext{
6 Jacke und Drewes (2004)

7 Die maximale Nutzungsdauer der Forstreifen ergibt sich aus Interviews mit Experten der Reifenindustrie (MÜLLER, 2002; FENSEL, 2002)
} 


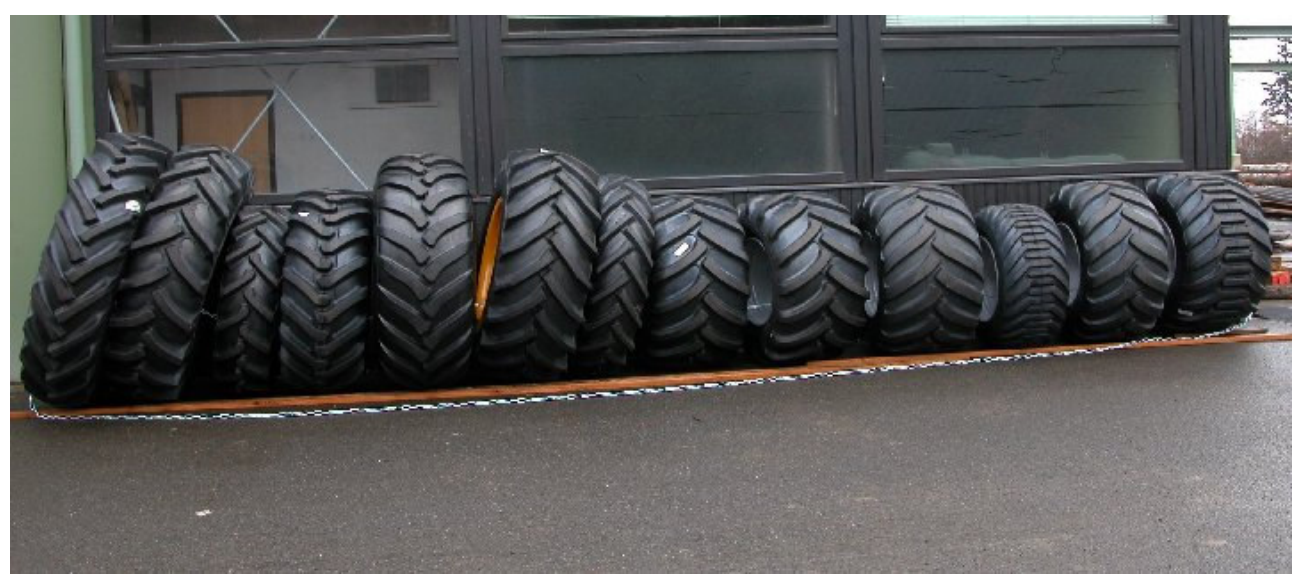

Abbildung 18: Reifenkonglomerat unterschiedlichster Dimensionen von der Firma Trelleborg vor der Göttinger Institutswerkstatt unmittelbar vor Bemessung der Druckverteilung 


\section{Konstruktion der Messtechnik}

\subsection{Stand der Forschung}

Zur Entwicklung einer geeigneten Messmethode bzw. Druckbemessungstechnik, erfolgte eine erste Kontaktaufnahme zu den Forschungsabteilungen der Reifenhersteller Trelleborg, Nokian, Pirelli, Continental und Goodyear.

Nokian und Trelleborg verwenden zur Ermittlung der Reifenaufstandsflächen ein sehr einfaches Verfahren, bei dem mittels Kohlepapier „Stempelabdrucke“ (s. Kap. 2.1) gemacht werden. Dieses Verfahren lässt aber keine Rückschlüsse auf die in der Kontaktfläche herrschenden Druckverteilungen zu.

Pirelli (Kraftfahrzeugreifenhersteller) hingegen bedient sich spezieller Folien, deren Aufbau aus mehreren Schichten von Farbkügelchen bestehen. Die im unbelasteten Zustand transparenten Kügelchen platzen erst bei Ausübung eines definierten Druckes auf, so dass ihr Farbinhalt entweichen kann. Je nach Intensität des Druckes variiert auch die Intensität der Farbe. Auf diese Weise lässt sich mit Hilfe von EDV-Programmen die Druckverteilung auswerten. Diese Folien können allerdings nur maximal viermal wiederverwendet werden.

Continental benutzt zur Ermittlung der Druckverteilung ein Verfahren, bei dem sich zwischen dem Reifen und der als Widerlager fungierenden Glasplatte eine Folie mit Prismenstruktur befindet, die durch das Aufdrücken ihre Reflexionseigenschaften ändert. Unter der Platte ist eine starke Lichtquelle und ein Bilderfassungssystem (Graustufenkamera) angebracht, das die unterschiedlichen Drücke als Grauwerte erkennt. Computergestützt wird dieses Bild weiterverarbeitet. Den Grauwerten werden Farben zugeordnet und diesen Farben dann - durch das Auswerten der Flächengröße und der Kenntnis der Radlast - Drücke.

Goodyear hat patentrechtlich geschützte Sensormatten entwickelt, die auf Basis der TekscanTechnologie die Aufstandsfläche und die Kontaktflächendruckverteilung wiedergeben. Diese Technik verwendet Goodyear lediglich im Bereich der Kraftfahrzeugreifenentwicklung.

Neben den bereits bestehenden Kontakten des Instituts für Forstliche Arbeitswissenschaft und Verfahrenstechnologie der Universität Göttingen (im Folgenden IFA genannt) zu der Technischen Universität München (Studienfakultät für Forstwissenschaft \& Ressourcenmanagement), der Fachhochschule Köln (Fachbereich Landmaschinentechnik) und der Fachhochschule Hildesheim/Holzminden/Göttingen (Fachbereich Naturwissenschaften und Technik) wurden zudem auch die auf dem Gebiet der „Bodenverdichtung“tätigen Universitäten Kiel und Hohenheim (Agrarwissenschaftliche Fakultäten) und die Fachhochschule Südwestfalen (Fachbereich Landbau und Agrarwissenschaften) involviert.

Die Universitäten Kiel/Hohenheim und die Fachhochschulen Südwestfalen/Köln messen Verdichtungen mit im Boden befindlichen Sonden ausschließlich für Ackerschlepperreifen in eigens konstruierten Bodenrinnen oder durch Bodenüberfahrten in Freilandversuchen.

Der Forstwissenschaftliche Fachbereich der Technischen Universität München hat ein Programm namens „ProFor“ zur bodenschonenden Befahrung entwickelt. Hierzu wurden Befah- 
rungsversuche auf unterschiedlichen Böden durchgeführt und das Verhalten der Bodensubstrate in Abhängigkeit von den Wassergehalten ermittelt. Im Rahmen der Weiterentwicklung dieses Programms wurde die Aufstandsfläche von 15 Forstreifen in Form von Stempelabdrucken untersucht.

Eine weitere in diesem Bereich forschende Projektgruppe mit Namen „Rad-Boden“der Agrargenossenschaft Burkhardswalde misst die Druckverteilung mit einem stationären Prüfstand auf festem Untergrund. Die Druckintensität wird mit in Silikon eingelagerten Drucksensoren (Fa. Kistler) ermittelt. Durch EDV-Programme können die Druckverteilung und die Aufstandsfläche berechnet und ausgewertet werden.

Die explizite Erforschung der Schnittstelle „Reifen-Boden“bzw. die Druckverteilung auf Kontaktflächen unter Forstreifen führte keine der genannten wissenschaftlichen Einrichtungen durch.

\subsection{Wahl der Messtechnik}

Das IFA beabsichtigte anfangs, die Messtechnik zur Druckverteilung in Eigenregie zu konstruieren. Deren Entwicklung hätte aber einen unverhältnismäßig hohen Aufwand ergeben. Nach Rücksprache mit Experten der Fachhochschule Hildesheim/Holzminden/Göttingen (Fachbereich Naturwissenschaften und Technik) verwarf man diesen Ansatz und suchte nach bereits bestehenden Marktlösungen.

Neben den durch die Firma Goodyear benannten Anbieter entsprechender Sensormesstechnik (Tekscan Inc.) wurden die Lösungen der Unternehmen Kistler Instrumente GmbH, Novel $\mathrm{GmbH}$ und Sartorius AG näher betrachtet.

Nach Auswertung aller Informationen und Ergebnisse fiel die Wahl der zu verwendenden Sensormesstechnik auf die US-amerikanische Firma Tekscan. Die Systeme der anderen Unternehmen erfüllten die im Rahmen der beabsichtigten Bemessungen von Forstreifen zu erbringenden Leistungsmerkmale und Anforderungen nicht. Zudem waren die Messsysteme der Anbieter in einem Kosten-Leistungsvergleich um ein Vielfaches teuerer, als die von Tekscan angebotene Lösung.

\subsection{Sensormesstechnik}

\subsubsection{Aufbau und Funktionsweise der Tekscan-Sensormatten}

Mit der hochauflösenden, computergestützten Messtechnik der US-amerikanischen Firma „Tekscan“ können die Druck- bzw. Kraftverteilung, welche auf die Fläche einwirken, innerhalb kürzester Zeit erhoben werden. Zur Ermittlung der Kontaktflächendruckverteilung wird der Sensor vom Typ MATSCAN/3150 verwendet (Abb. 19). 


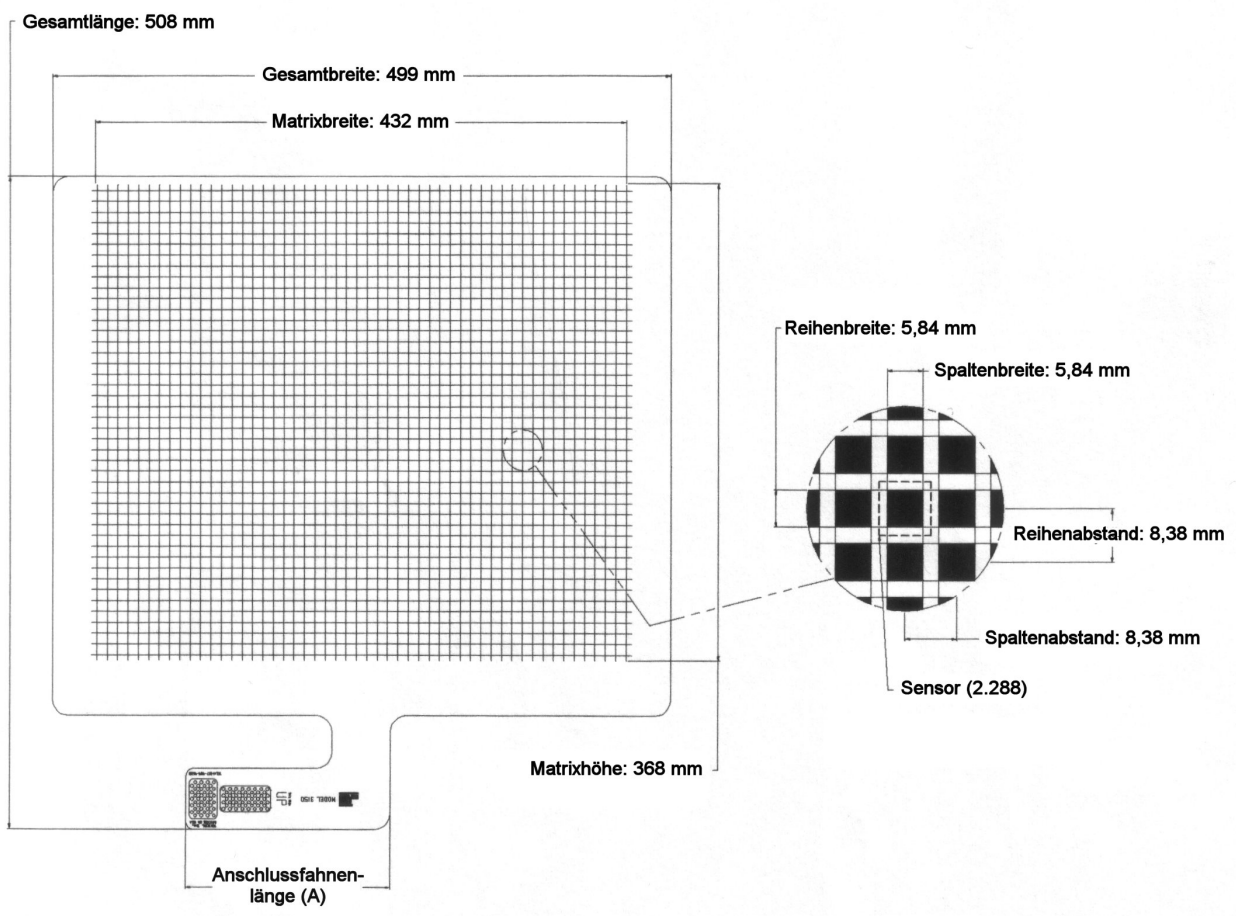

Abbildung 19: Geometrischer Aufbau des Sensors MATSCAN/3150 (verändert nach TEKSCAN, 2001a)

Der MATSCAN/3150-Sensor (Breite: $43,2 \mathrm{~cm}$ x Tiefe: $36,8 \mathrm{~cm}$ ) besitzt einen geometrischen Messbereich von 1589,76 $\mathrm{cm}^{2}$, über den 2288 Sensorzellen verteilt sind.

Insgesamt werden vier Sensormatten benötigt, um das Spektrum sämtlicher Reifendimensionen (bis max. 800 mm Reifenbreite) abzudecken. Die vier zusammengefügten Sensormatten und ihre insgesamt 9152 Sensorzellen bilden in vertikaler und horizontaler Richtung eine Matrix. Jede Sensorzelle stellt hierbei einen Pixel dar, dessen Abmessungen Abbildung 19 zu entnehmen sind.

Die Sensormatte besteht aus zwei sehr dünnen, flexiblen Polyesterfolien, auf denen Leiterbahnen gedruckt sind (Abb. 20). Auf einer der Innenseiten der Sensormatte befinden sich die Spalten, auf der anderen Seite die Reihen. Durch das Übereinanderlegen beider Folien entsteht an jedem Kreuzpunkt der Matrix ein Sensorelement (Sensorzelle), dessen Dicke $<1 \mathrm{~mm}$ ist. Die elektrische Leitfähigkeit wird durch eine halbleitende Tinte, die die Sensorzelleninnenseiten benetzt, hergestellt (COMPUTER MESSTECHNIK VERTRIEB CMV HOOVEN, 2001).

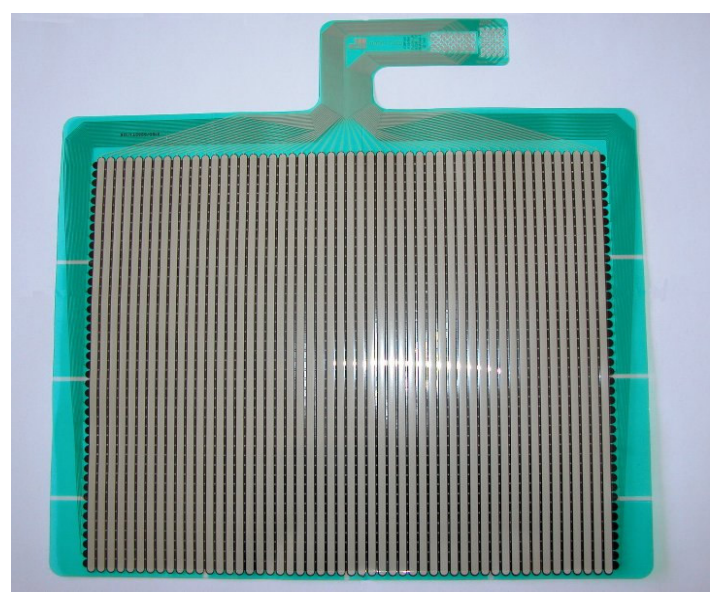

Abbildung 20: Tekscan-Sensormatte

Das Schaltbild zeigt den elektronischen Aufbau in vereinfachter Form (Abb. 21). Jeder Kreuzpunkt der Matrix stellt einen variablen Widerstand dar, dem eine 8-Bit Wertigkeit (256 Stufen) zugeordnet ist. Im unbelasteten Zustand (keine Kraftausübung auf der Sensormattenoberfläche) ist der Widerstand am größten. Das Messprinzip besteht darin, dass in jeder Sensorzelle 
zwei Widerstände lokalisiert sind, die jeweils reihen- und spaltenweise mit einer Ausgangsspannung beaufschlagt werden. Beide Widerstände verringern sich bei mechanischer Belastung - und zwar proportional zur Auflast. Reihen- und Spaltenwiderstand werden gemittelt und geben damit eine analoge Information zur punktuellen Last. Über einen prozessgesteuerten Multiplexer ${ }^{8}$ werden die Reihen und Spalten von links nach rechts und von oben nach unten abgetastet und die Signale zu einem Gesamtbild verknüpft.

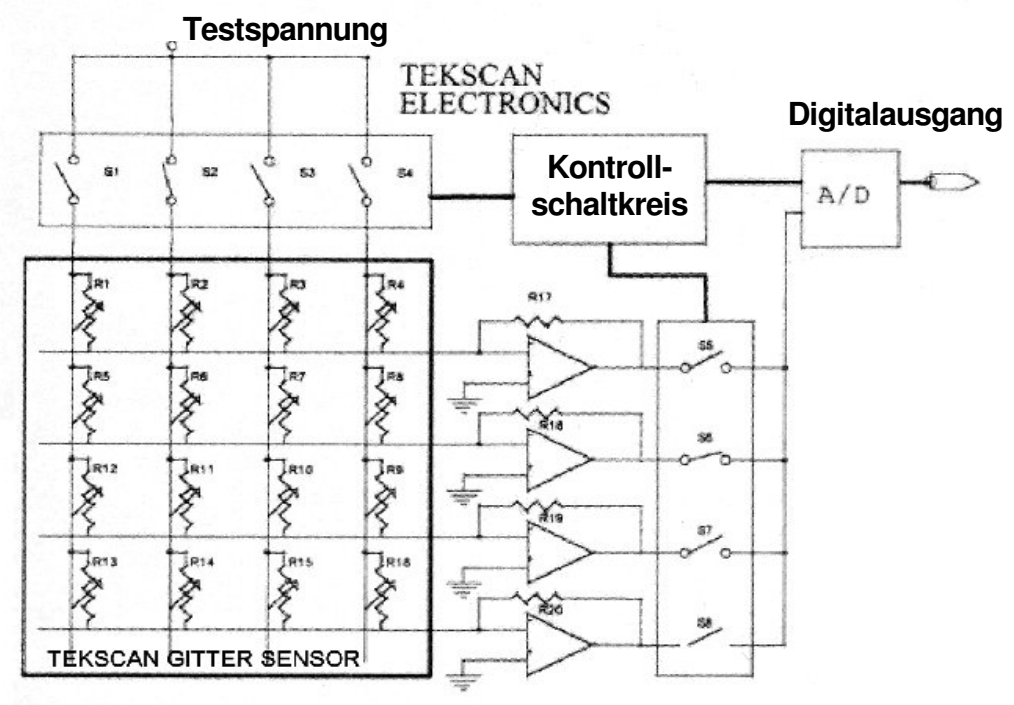

Abbildung 21: Symbolischer Schaltkreis einer Tekscan-Sensormatte (verändert nach TEKSCAN, 2001a)

Die aufgezeichneten Daten werden jeweils einzeln mit einem Bügelgriffanschluss (genannt Handle, Abb. 22) eingelesen und über eine Messkartenbox an den PC weitergeleitet. Um ein Gesamtbild des Reifenabdruckes zu bekommen, werden die Daten jeder einzelnen Sensormatte eingelesen und im ASCIIFormat $^{9}$ abgespeichert. Die weitere Datenverarbeitung erfolgt mit mehreren vom IFA entwickelten Programmen, die die Daten jeder einzelnen Sensormatte zu einem Gesamtbild zusammenfassen und mit denen sowohl graphische als auch

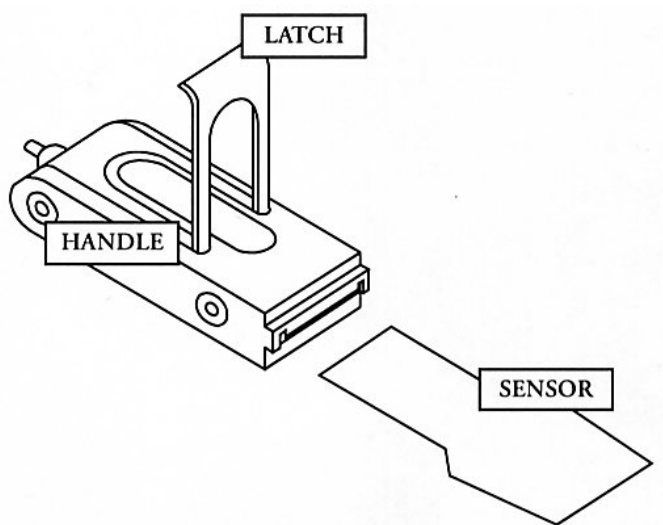

Abbildung 22: Demonstrationsübersicht des Gebrauchs eines Handles zum Anschluss einer Tekscan-Sensormatte (TEKSCAN, 2001b) statistische Auswertungen ermöglicht werden (s. Kap. 6.2).

\footnotetext{
Komponente, die eine Leitung für die gleichzeitige Übertragung mehrerer Signale nutzt.

9 American Standard Code for Information Interchange
} 


\subsubsection{Equilibrieren der Sensormatten}

Mit der Equilibrierung soll das Herstellen eines Kraftausgleichs bzw. Gleichgewichtszustandes erreicht werden. Auf der gesamten Fläche des Messbereiches der Sensormatte wird ein einheitlicher Druck ausgeübt. Ziel ist es, jede einzelne Sensorzelle gleichmäßig zu belasten. Hierzu wurde vom IFA eine spezielle Equilibriervorrichtung (engl. Bladder) gefertigt. Der Bladder (Abb. 23) besteht aus einem Stahlgehäuse, das von oben und von unten jeweils von einer $4 \mathrm{~cm}$ dicken Stahl-

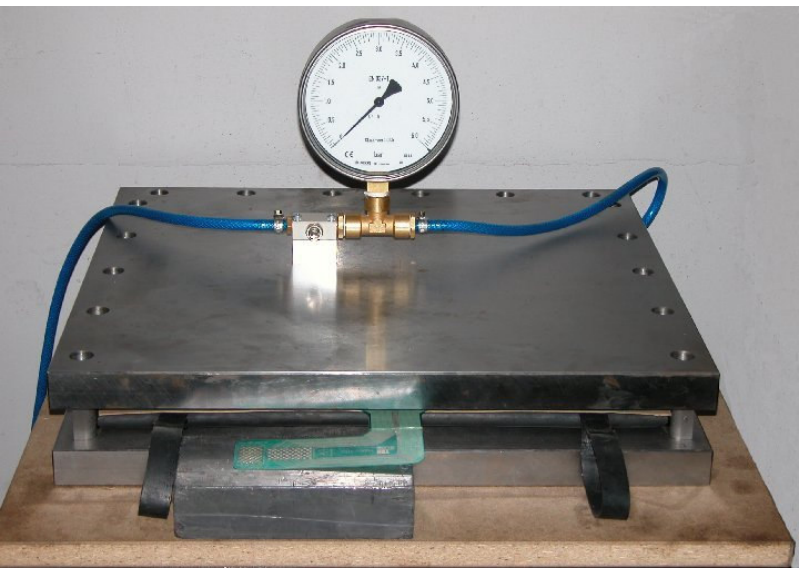

Abbildung 23: Bladder platte (Breite: $59,5 \mathrm{~cm}$, Tiefe: $54,5 \mathrm{~cm}$ ) begrenzt wird. Innerhalb dieses Stahlgehäuses befindet sich ein speziell angefertigtes Mini-Hebekissen ${ }^{10}$. Ein Sicherheitsventil und ein Manometer garantieren eine kontinuierliche und kontrollierte Druckluftzufuhr. Die ebene, glatte Oberfläche des Hubkissens dehnt sich plan über den gesamten Messbereich der Sensormatte aus. Versuche haben ergeben, dass das Equilibrieren in 0,5-bar-Stufen bis zu einer Maximalbelastung von 4 bar empfehlenswert ist. Über die Menüfunktion „Equilibrate“der I-Scan-Software (Version 5.01) werden die einzelnen Druckstufen in Equilibrierdateien abgespeichert. Der Prozess des Equilibrierens ist die Voraussetzung für den nächsten Schritt, das Kalibrieren.

\subsubsection{Kalibrieren der Sensormatten}

Bei dem Kalibrieren werden die über den (im Folgekapitel 4.4 vorzustellenden) Radlastsimulator hydraulisch erzeugten Radlasten - mittels Kraftaufnehmer - und die über die Sensormatten aufgenommenen Werte verglichen. Zur Minimierung der Abweichungen der Messwerte wurden die Messgeräte untereinander abgestimmt. Hierzu wurde jede einzelne Sensorzelle mit einem Stempel, der den gesamten Messbereich der Sensormatte abdeckte und sich zwischen dem Reifen und der jeweiligen Sensormatte befand, definitionsgemäß belastet. Durch die Wahl der physikalischen Einheit „bar“ im Programmmenü und einer zweiminütigen Setzungsdrift der Sensorzellen (die sensorspezifisch bedingt war und eingehalten werden musste), wurden die Laststufen über die I-Scan-Software (Version 5.01) erfasst und per integrierte Kalibrierroutine als Kalibrierpunkt eingestellt und abgespeichert. Mit der Kalibrierung ist jede Sensormatte in der Lage, bei den Messungen der Kontaktflächendruckverteilung die auftretenden Lasten bzw. Drücke zuzuordnen und eine präzise Erhebung der Daten zu gewährleisten.

10 Hersteller Vetter (Typ V 18, Betriebsdruck: 8 bar, Breite: $52 \mathrm{~cm}$ und Tiefe: $47 \mathrm{~cm}$ ) 


\title{
4.4 Entwicklung des Radlastsimulators
}

Die im Folgenden genannten Eigenschaften eines Reifens (bzw. eines Rades), die insbesondere für die Messungen der Kontaktflächendruckverteilung bedeutsam sind, waren für die Konstruktion des Radlastsimulators entscheidend:

\author{
1. Radlast / Auflast \\ 2. Reifenfülldruck \\ 3. Steifigkeit der Karkasse (LI/PR) \\ 4. Karkassenbauweise (Diagonal- oder Radialbauweise) \\ 5. Nennbreite und $H / B$-Verhältnis \\ 6. Rad-Durchmesser \\ 7. Profilschema
}

Die fünf Eigenschaften 3 bis 7 sind konstruktiv vorgegeben, allein die Radlast und der Reifenfülldruck sind variabel. Der Radlastsimulator ist so konzipiert, dass die Kontaktflächendruckverteilung durch Aufbringung von fünf definierten Radlasten (10 kN, 20 kN, 30 kN, 40 kN, 50 kN) und sieben Reifeninnendrücken (0,5 bar, 1,0 bar, 1,5 bar, 2,0 bar, 3,0 bar, 4,0 bar, 5,0 bar) in Form einer statischen Messung ermittelt wird.

Die Bandbreite an Maschinenmassen, die ebenfalls aus der Marktanalyse abgeleitet werden konnten, reichen von $\sim 5 \mathrm{t}$ bis $30 \mathrm{t}$ (s. auch MATTHIES, 2002; KURATORIUM FÜR WALDARBEIT UND FORSTTECHNIK, 2001). Ausgehend von den Landwirtschaftlichen Schleppern mit Forstausrüstung, die die untere Grenze der Maschinenmassen bilden, erfolgt eine weitere Zunahme in der Reihenfolge Schlepper, Forwarder bis zu den Harvestern mit einer Obergrenze von ca. $18 \mathrm{t}$. Hierbei handelt es sich lediglich um die Eigenmassen der Maschinen ohne deren mögliche Zuladung, die aber z. B. bei Forwardern von $8 t$ bis $14 t$ betragen kann. Somit wird mit einem Massemaximum von $30 \mathrm{t}$ das Spektrum sämtlicher zurzeit forstüblicher Maschinen und deren Ladekapazitäten abgedeckt. Es darf deshalb davon ausgegangen werden, dass unter Berücksichtigung der Anzahl der Räder (4-, 6- und 8-Rad-Maschinen), der Schwerpunktlagen der Maschinen, der Arbeitspositionen und den involvierten Massen für die genannten Maschinenkategorien, die zu erwartenden Kräfte sich in den Grenzen von $10 \mathrm{kN}$ bis $50 \mathrm{kN}$ bewegen und der Bereich dieser definierten Laststufen die auftretenden Radlasten sämtlicher Maschinentypen abgedeckt und somit für die Untersuchung der Kontaktflächendruckverteilungen einheitliche Messbedingungen geschaffen werden.

Die Festlegung der Reifenfülldrücke erfolgte einerseits auf der Grundlage von Herstellerangaben und andererseits sollte insbesondere das Kontaktflächendruckverhalten der Reifen bei sehr geringen Innendruckstufen (z. B. 0,5 bar, 1,0 bar) untersucht werden.

Die Konstruktion und die Anfertigung des Radlastsimulators hat das IFA in Eigenregie vorgenommen. Die Hauptkomponenten/Bauteile des Radlastsimulators werden in Abbildung 24 beschrieben. 


\section{Abmessungen:}

Breite gesamt: $1,50 \mathrm{~m}$ Höhe gesamt: $3,85 \mathrm{~m}$ (inkl. Schubkolben-Hydrozylinder) Länge gesamt: $2,40 \mathrm{~m}$ Eigenmasse: $1.800 \mathrm{~kg}$

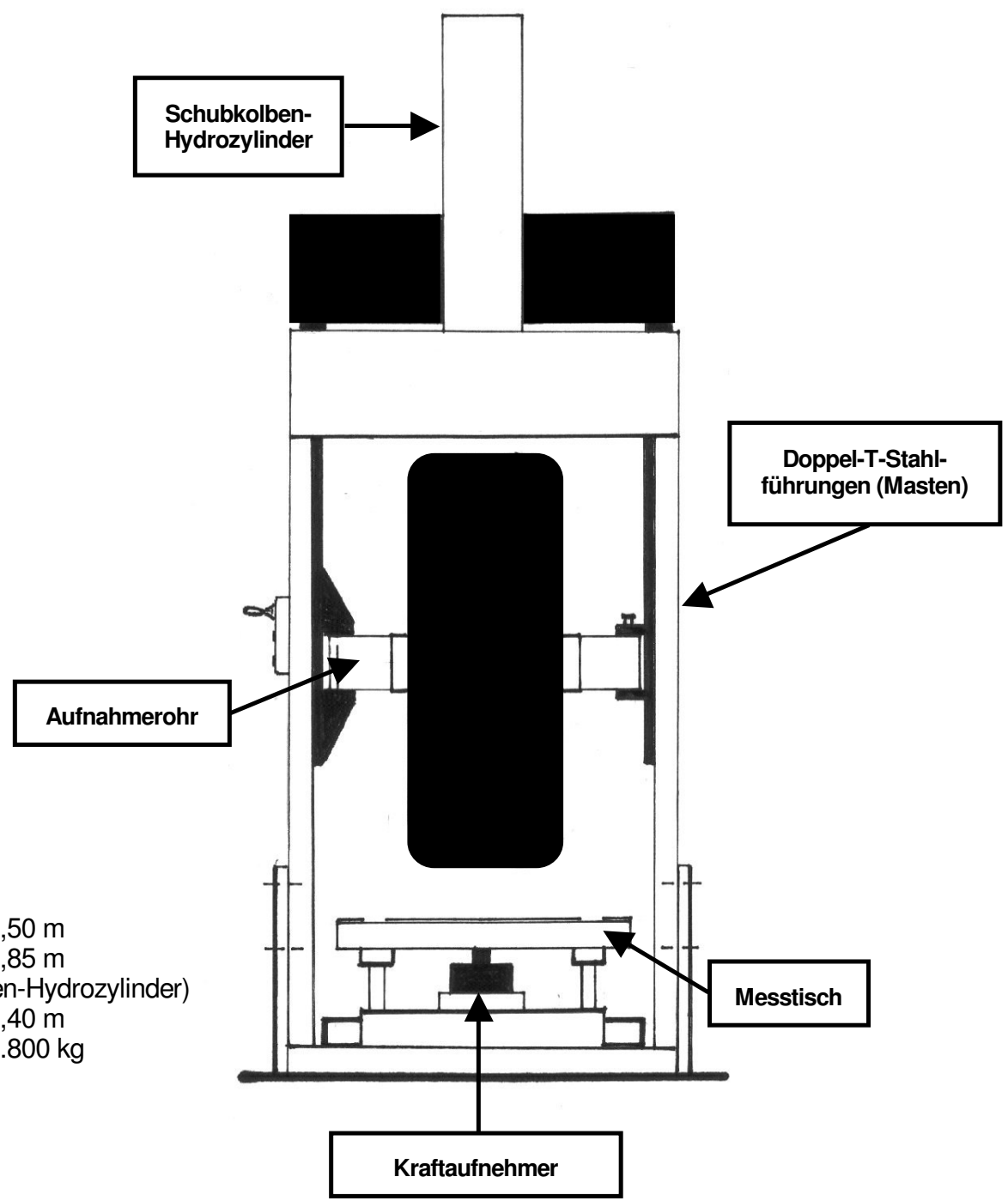

Abbildung 24: Schematische Zeichnung des Radlastsimulators und Beschreibung seiner Bauteile

Die Abbildung 25 zeigt den Radlastsimulators in der Werkhalle des IFA. Um die Bemessung sämtlicher Räder zu garantieren, richtet sich der Durchmesser des Aufnahmerohrs nach dem kleinsten Nabenausschnitt (Felgenausschnitt zur Zentrierung der Felge mit Reifen bzw. zum Aufsetzen der Felge auf die Radachse) der auf den Maschinentypen zum Einsatz kommenden Felgen. Danach ergibt sich ein Durchmesser für das in Abbildung 26 dargestellte Aufnahmerohr von $15 \mathrm{~cm}$ (Länge: $105 \mathrm{~cm}$, Wandung: $2 \mathrm{~cm}$ ). Mit einem entsprechend gefertigten Adapter, dessen Anschlussmaße sich an den am häufigsten vorkommenden Lochkreisdurchmessern der Felgen orientieren, können alle Felgengrößen bzw. deren Nabenausschnitte und Schraublochbohrungen aufgezogen werden. Dieser Adapter erlaubt einerseits eine schnelle Montage der Räder und andererseits über die $\sim 45^{\circ}$ schwenkbare Aufnahmevorrichtung (Abb. 27) eine problemlose Fixierung und Arretierung der Räder auf dem Aufnahmerohr des Radlastsimulators. 


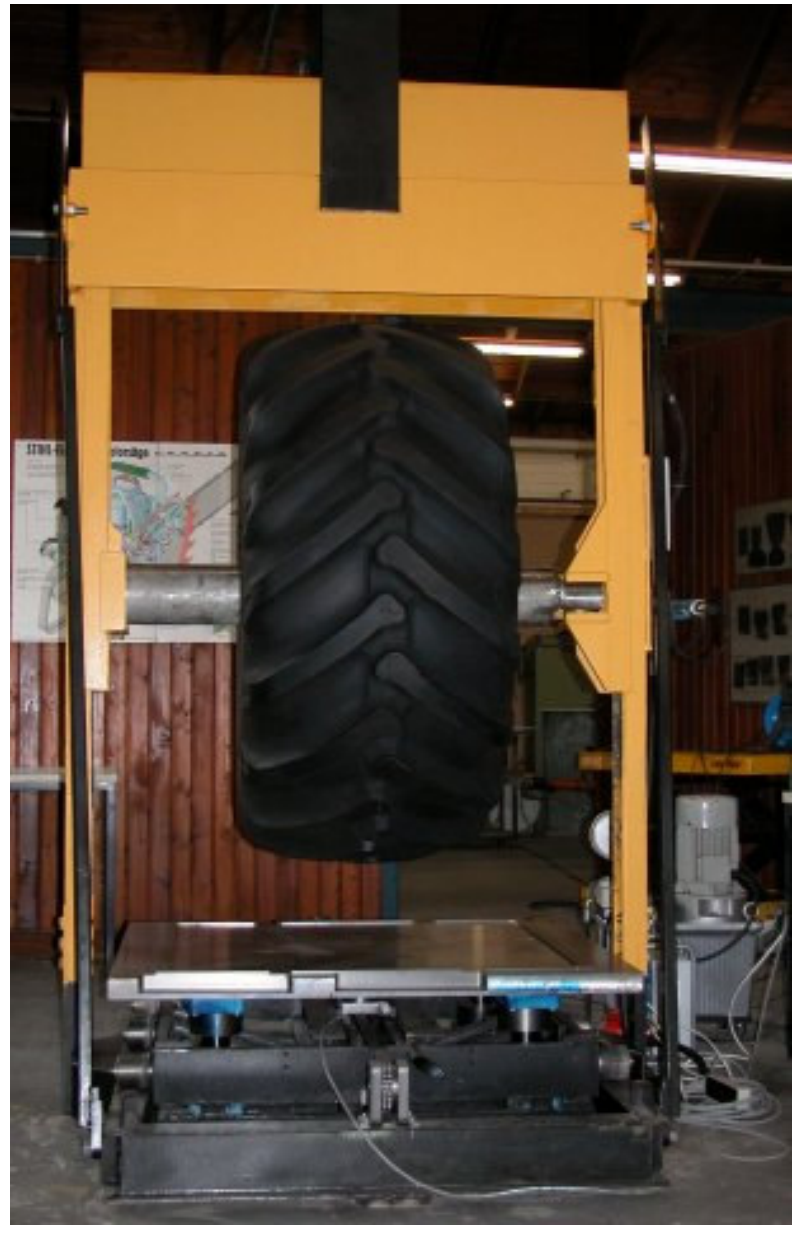

Abbildung 25: Gesamtaufnahme des Radlastsimulators

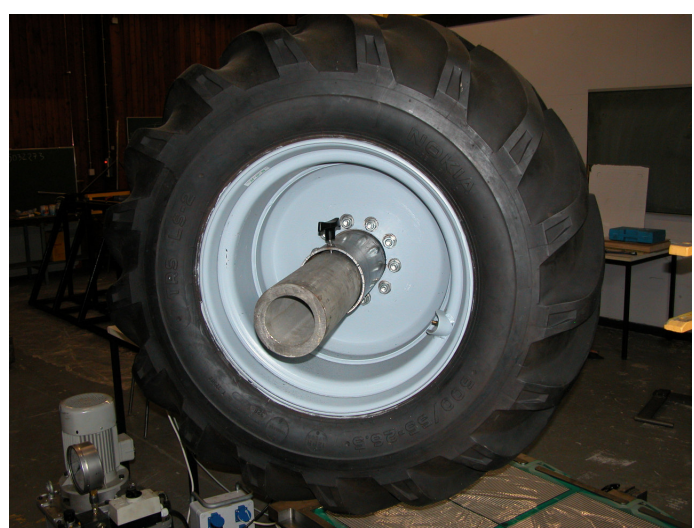

Abbildung 26:Aufnahmerohr und Adapter mit Reifen

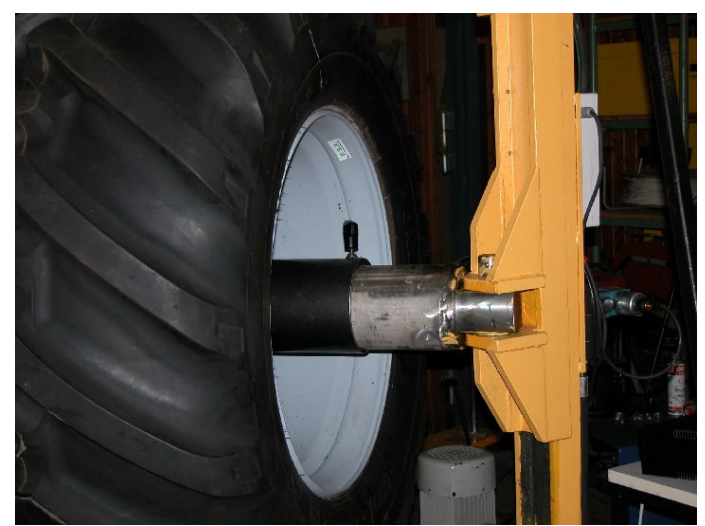

Abbildung 27:Aufnahme- und Schwenkvorrichtung für die Räder 
Über das Hydraulikaggregat ${ }^{11}$ und dem Schubkolben-Hydrozylinder ${ }^{12}$ werden die einzelnen Radlasten simuliert. Mit einem Rückschlagventil kann der Druck in jeder Position gehalten werden, wobei das montierte Rad in Doppel-T-Stahlführungen (Masten) senkrecht geführt wird. In der Abbildung 28 ist ein detaillierter Hydraulik-Schaltplan zu sehen, der das Funktionsprinzip dieser Baueinheiten des Radlastsimulators beschreibt.

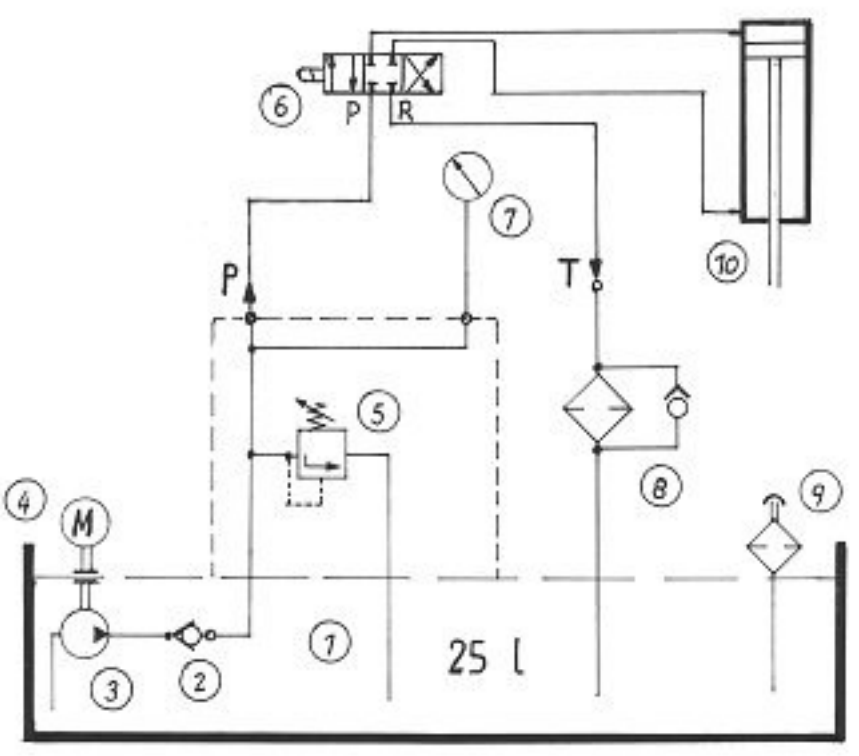

1. Flüssigkeitsbehälter (Hydrauliköl)

2. Rückschlagventil

3. Hydraulikpumpe

4. Motor: $3 \mathrm{~kW}, 400 \mathrm{~V}, 50 \mathrm{~Hz}$

5. Druckregelventil mit Elektromagnet

6. 4/3 Wegeventil mit Elektromagnet

7. Manometer mit Rückschlagventil

8. Filter

9. Einfüllstutzen mit Filter

10. Doppeltwirkender Zylinder mit einseitiger Kolbenstange

Abbildung 28: Hydraulik-Schaltplan des Radlastsimulators (STREEß, 2003)

Das Ein- und Ausfahren des SchubkolbenHydrozylinder wird mit einem Koordinationsschalter gesteuert. Zur Ermittlung des Kontaktflächendrucks werden dann die Räder auf einen Messtisch gedrückt. Unterhalb dieses Messtisches befindet sich zentriert ein Kraftaufnehmer ${ }^{13}$, der über einen Messverstärker ${ }^{14}$ die simulierte Radlast wiedergibt. Da die Erfassung der Radlast unterhalb der Kontaktfläche erfolgt, ist in dieser die Radmasse bereits enthalten.

Der Messtisch ist 1,20 m breit und $1 \mathrm{~m}$ tief. Auf

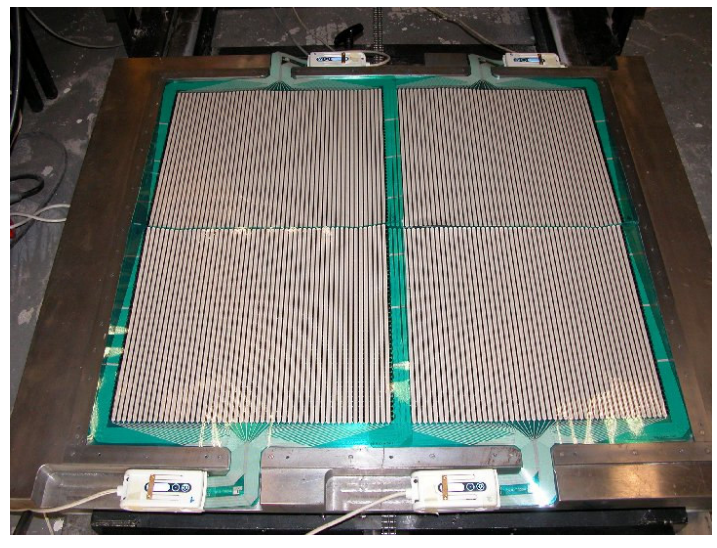

Abbildung 29:Messtisch mit eingefassten Sensormatten diesem eingefasst, befinden sich die Sensormatten, mit denen die Kontaktflächendruckverteilung unter den Reifen untersucht wird (Abb. 29). Bei diesen Messungen auf hartem Untergrund können Informationen zum Fahrverhalten des Reifens auf befestigten Waldwegen und Straßen erhoben werden. Eine Interpretation der Druckverteilung beschränkt sich hierbei nur auf die Stollenkontaktfläche. Die Stollenzwischenräume, welche die Last mit abstützen und den insgesamt aufgebrachten Druck mindern, wer-

\footnotetext{
11 Typenbezeichnung RBA 125/80-600; Qmax 8 I/min bei pmax 160 bar

12 Kolben $125 \mathrm{~mm}$, Stange $80 \mathrm{~mm}$, Hub $600 \mathrm{~mm}$

13 Hottinger Baldwin Messtechnik (HBM), Typenbezeichnung: C2, Nennmessbereich: 10 kN

14 HBM, Typenbezeichnung: MVD 2555
} 
den nicht berücksichtigt. Durch ein Einsinken des Reifens auf weichem Untergrund ist es möglich, die Druckverteilung für die Stollen und deren Zwischenräume zu erfassen. Diese als Ganzes erhobenen Kontaktflächenabdrucke sind demnach für die direkte Fragestellung der Verteilung des Druckes besser geeignet. Zur Erhebung dieser Kontaktflächenabdrucke, wurde eine Box angefertigt (Länge: 1,85 m, Breite: 0,92 m, Höhe: 0,25 m), die mit einem Substrat (in diesem Fall Sand mit einer Korngröße $<1 \mathrm{~mm}$ ) bis zu einer definierten Höhe von $20 \mathrm{~cm}$ aufgefüllt wird (Abb. 30). Diese Box kann per Kettenführung auf Trägerschienen des Radlastsimulators zum Befüllen und zum Abziehen bzw. Glätten des Substrats waagerecht verschoben werden. Sollte sich die Aufstandsfläche des Reifens in der Länge über den Messbereich der Sensormatten ausdehnen, ist eine

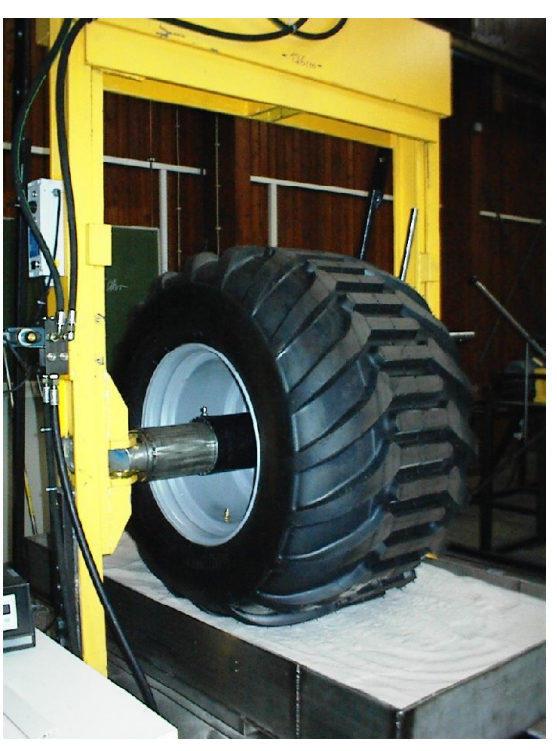

Abbildung 30:Sandbox mit Füllmaterial

Vollerhebung der Kontaktfläche des Reifens durch diese Vorrichtung ebenfalls gewährleistet.

\subsection{Entwicklung der Walkmaschine}

Bei den zu bemessenden Rädern handelte es sich um solche mit fabrikneuen Reifen. Um einen Bezug zu den in der forstlichen Praxis herrschenden Einsatzbedingungen der Reifen zu schaffen, hat das IFA eine weitere Maschine konstruiert (Abb. 31), die in der Lage ist, den Reifenunterbau (Karkasse) durch ein entsprechendes Walken ${ }^{15}$ der Reifen elastischer zu machen.

Abmessungen:

Breite: $2,83 \mathrm{~m}$

Länge: $2,83 \mathrm{~m}$

Höhe: $1,10 \mathrm{~m}$

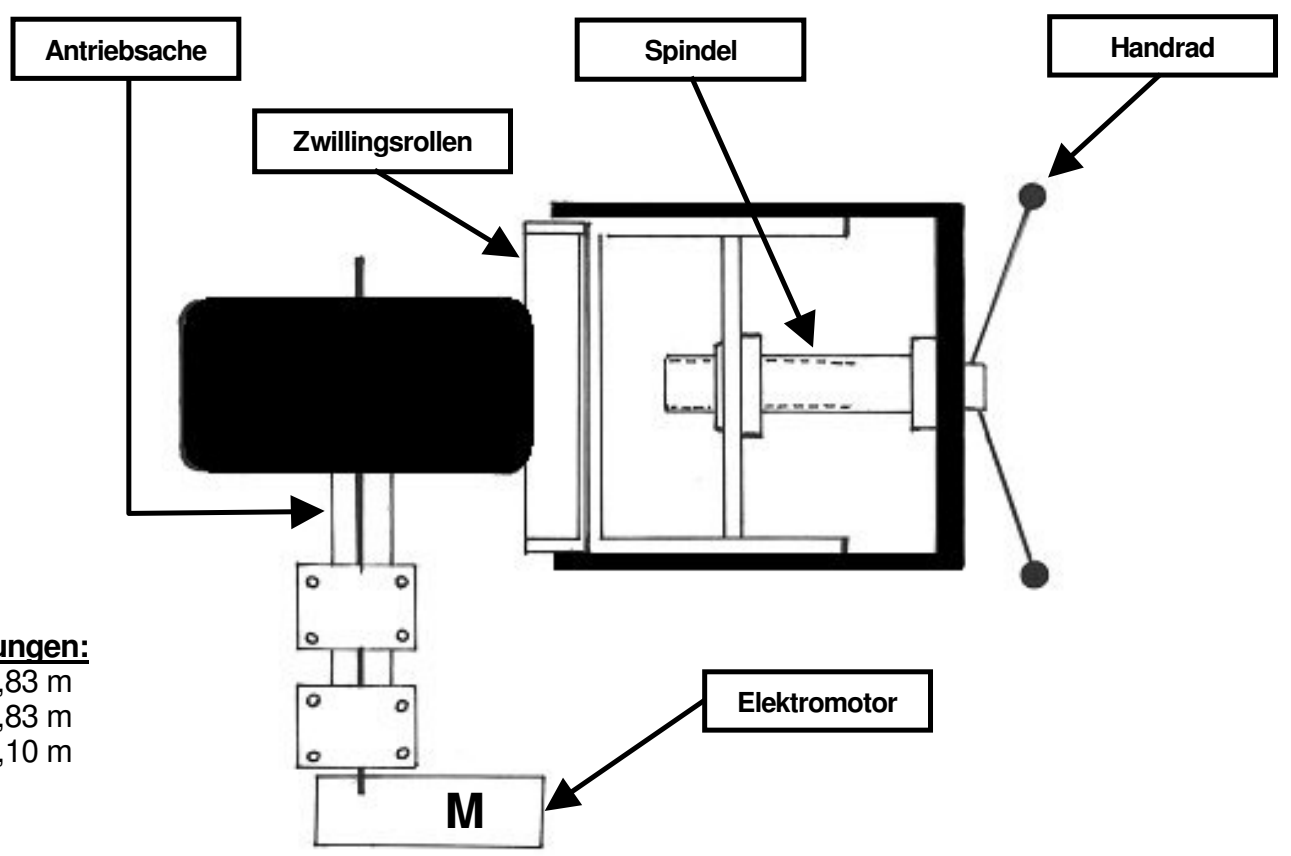

Abbildung 31: Schematische Zeichnung - Aufsicht der Walkmaschine mit Beschreibung der Bauteile

15 Verformungsarbeit des Reifens beim Durchlaufen des Latsches. 
Zwei Effekte sind es, die es notwendig machen, einen Reifen zu walken bzw. einzufahren:

1. Brechen von Schussfäden und Luftabführungsfäden, die eine charakteristische Reifenverformung einschränken

Reifen werden aus Corden gebaut (s. Kap. 1.3.1). Das heißt, die einzelnen Gewebelagen im Reifen bestehen hauptsächlich aus Kettfäden. Es sind nur vereinzelt Schussfäden (meist geringerer Festigkeit) als Verarbeitungshilfe vorhanden. Diese Schussfäden werden nach dem Gummieren des Gewebes mit Hilfe von Brecherwalzen angerissen, um die Verformbarkeit des fertigen Reifens nicht zu behindern. Ebenso werden auf die gummierte Gewebelage feine Luftabführungsfäden aufgebracht, die, wie der Name andeutet, eingeschlossene Luft während des Fertigungsprozesses abführen. Diese versteifenden Strukturen verlieren ihre Festigkeit nach wenigen Umdrehungen des Rades unter Last - innere Spannungen werden beim Bewalken abgebaut.

\section{Wachstum der Nylonkarkasse unter Last (Fülldruck) bzw. Wärmeentwicklung}

Als Karkassenmaterial beim Reifenbau werden oft Festigkeitsträger aus Nylon verwendet. Dieses Material ist hochfest, kriecht (verformt) aber unter Last. Circa $80 \%$ des Wachstums (hiermit ist das Recken/Verformen der Corde unter Druck gemeint) sind statisch nach 24 Stunden unter Druck abgeschlossen, weitere $15 \%$ nach 48 Stunden. Die verbleibenden ca. $5 \%$ kommen im restlichen „Reifenleben“ - Änderung des Reifenverhaltens durch Abrieb, Verschleiß und/oder Alterung - hinzu (KRIEGER, 2003).

Durch Erwärmung und Betrieb wird dieser Prozess beschleunigt. Nylon hat die Angewohnheit, bei Temperaturen über $80^{\circ} \mathrm{C}$ zu schrumpfen und seine Molekularstruktur zu verändern ${ }^{16}$. Wird der Reifen beim Durchlaufen dieser Temperatur von oben nach unten verformt, bleibt diese Verformung bestehen (Memory-Effekt), bis der Reifen wieder diese Temperatur überschreitet (KRIEGER, 2003). Beim Konditionieren des Reifens wurde eine Temperatur von $80^{\circ} \mathrm{C}$ nicht überschritten. Zudem unterlag der Reifen hinsichtlich seiner Temperaturentwicklung einer permanenten Überwachung durch ein Infrarot-Strahlungspyrometer ${ }^{17}$.

Eine Reifenverformung zur Realisierung von Aufstandsflächen, wie sie im praktischen Einsatz zu erwarten ist, wird durch die Umsetzung dieser zwei Effekte ausreichend gewährleistet. In Abbildung 32 ist die Walkmaschine in der Werkhalle des IFA zu sehen.

${ }^{16}$ Bei Temperaturen von über $120^{\circ} \mathrm{C}$ würde ein Reifen irreversibel zerstört werden.

17 Hersteller: Heitronics (Typ: KT 19, Spektralbereich: 8 - $14 \mu \mathrm{m}$, Messbereich: -50 .. 1500 C) 


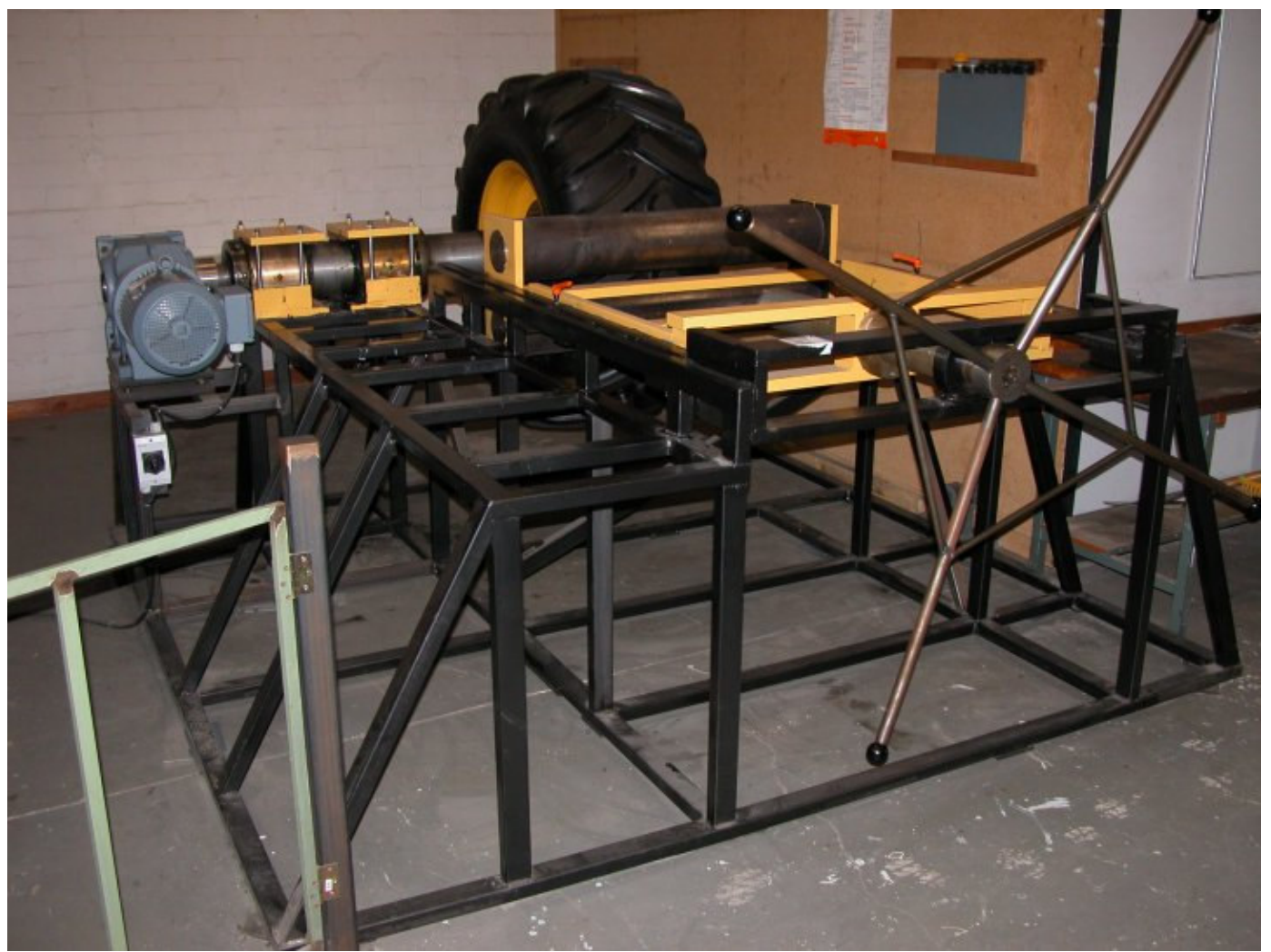

Abbildung 32: Gesamtaufnahme der Walkmaschine

Zum Bewalken werden die Räder an einen Adapter montiert, der mit der Antriebsachse (Länge: 1,34 m) verbunden ist. Die Achse wird direkt über einen Elektromotor ${ }^{18}$ angetrieben, der in seiner Auslegung die für alle Raddimensionen erforderlichen Drehmomente aufbringt (Abb. 33). In Abhängigkeit der Durchmesser der Räder (kleinster Durchmesser: 1,15 m - größter Durchmesser: $1,81 \mathrm{~m}$ ) wurde eine Spindel (Länge: $83 \mathrm{~cm}$ ) angefertigt, mit der zwei freidrehende Rollen (Zwillingsrollen: Länge 1,12 m,

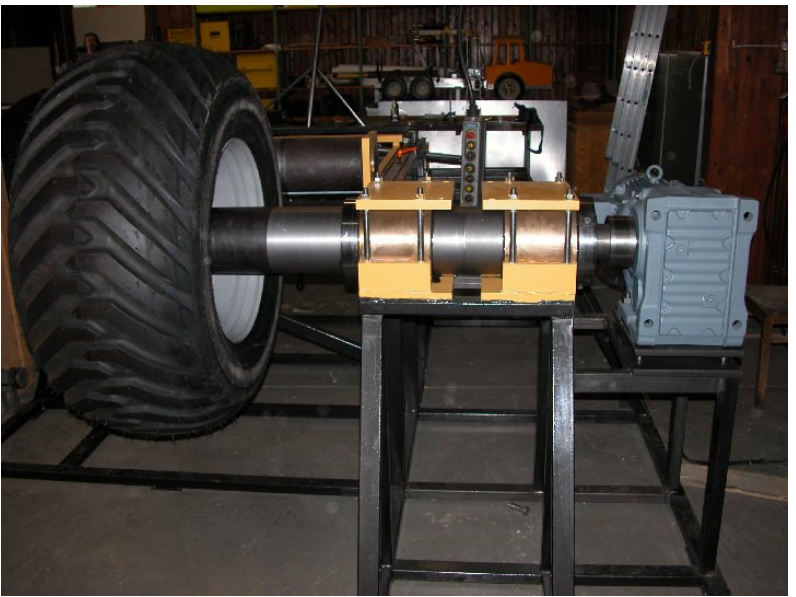

Abbildung 33:Elektromotor mit Antriebsachse Durchmesser $20 \mathrm{~cm}$, Rollenabstand $25 \mathrm{~cm}$ ) als Widerlager manuell (per Handrad) in waagerechter Position an den Reifen gepresst werden können. Die zu bemessenden Reifen werden mit einem Reifeninnendruck von 2 bar befüllt und auf $80 \%$ ihrer Nennhöhe eingedrückt. Bei dieser Begrenzung handelt es sich um eine Vorsichtsmaßnahme, die mögliche Schäden an den leihweise zur Verfügung stehenden Reifen vermeidet. Durch das Andrücken der Rollen wird Kraft in die Karkasse gebracht und während des Walkvorgangs kommt es zum Bruch der Schussfäden und Luftabführfäden - der Reifen wird konditioniert (Abb. 34).

18 Hersteller: SEW (Typ: KA97B DV132 MA-TF, P = 7,5 kW, M = 2830 Nm; Drehzahl: 25 upm, $\mathrm{f}_{\mathrm{B}}=1,50$ 
Es gibt keine eindeutige Definition des Prädikats „Eingefahren“für Reifen. Der Reifenhersteller Trelleborg erklärt z. B. einen Reifen als „eingefahren“, wenn dieser, montiert auf einer Maschine, eine Strecke von $10 \mathrm{~km}$ zurückgelegt hat. MATTHIES UND ZIESAK (2001) haben in den von innen durchgeführten Untersuchungen einen Reifen nach fünfzigmaligem Einfedern als bewalkt erklärt. Nach Aussagen der Forschungs- und Entwicklungsabteilung Continentals ist eine Laufstrecke von $5 \mathrm{~km}$ als ausreichend anzusehen.

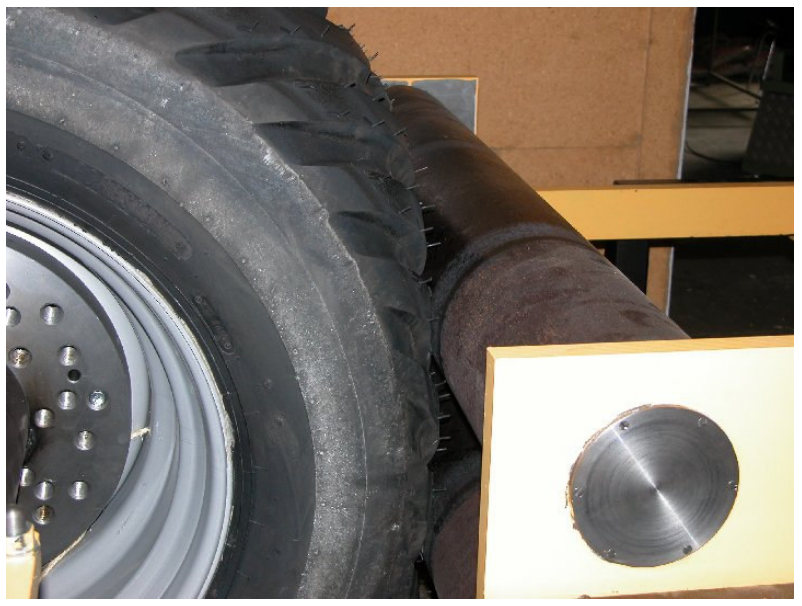

Abbildung 34:Walken des Reifens durch Anpressen

Seitens des IFA wird angesichts fehlender, allgemein verbindlicher Vorgaben eine unter den Simulationsbedingungen "gefahrene"Strecke von 5 km für ein Durchwalken der Karkasse als ausreichend gewertet.

Die radspezifische Laufzeit auf der Walkmaschine wird aus dem dynamischen Rad-Radius ${ }^{19} r_{d}$ berechnet. Hierzu wird der Umfang $U$ eines jeden Reifens mittels der Formel $U=2{ }^{*} \pi{ }^{*} r_{d}$ errechnet und mit der Drehzahl des Elektromotors (25 upm) multipliziert. Die definierte Strecke von 5000 m dividiert durch „Meter pro Minute [ $\mathrm{m} / \mathrm{min}]$ “ ergibt schließlich die spezifische Laufzeit des Reifens in „Minuten [min]“. Per Zeitschaltuhr wurde die exakte Einhaltung der Laufzeit gesteuert.

\subsection{Forschungsnetzwerk}

Die Bauzeit des Achslastsimulators und der dazugehörigen Walkmaschine inklusive entsprechender Funktionstests fand in dem Zeitraum von Januar 2002 bis März 2003 statt. Die Entwicklung und Umsetzung der Apparaturen hätte nicht ohne finanzielle Unterstützung realisiert werden können. Das Projekt PrAllCon war ein Teilprojekt eines innerhalb vom Ministerium für Schule, Weiterbildung und Forschung sowie dem Ministerium für Umwelt und Naturschutz, Landwirtschaft und Verbraucherschutz des Landes Nordrhein-Westfalen ins Leben gerufenes Forschungsvorhaben (Optimierung von Informations- und Materialfluss unter Simulationsbedingungen - OPTIMUS), dessen Schwerpunkt in der Verbesserung der Logistik des Rohholzes vom Erzeuger hin zum Be- und Verarbeiter lag. Da jeder logistische Prozess des Rohholzes mit dessen Ernte und v. a. mit dessen Transport verbunden ist, und dieser Transport sowohl heute als auch in absehbarer Zukunft die Bewegung motorgetriebener Maschinen auf Rädern voraussetzt, standen insbesondere deren Auswirkungen auf die Bodenökologie und im Detail ihre Kontaktflächendruckverteilungen im Interesse der Untersuchungen. Die im Rahmen des Projekts PrAllCon gesammelten Informationen und Geräte sollten deshalb genutzt werden, um besagte Kontaktflächendruckverteilungen in gesteuerter Abhängigkeit von Radlast und Fülldruck zu bestimmen.

19 Ist der Abstand zwischen der Radachse und der Kontaktfläche eines abrollenden Rades/Reifens $\left(r_{d}\right)$. 
Neben den von den Reifenherstellern als unentgeltliche Sachmittelleistung zur Verfügung gestellten Forstreifen bestand während der gesamten Laufzeit des Projekts PrAllCon Kontakt zu Experten (i. d. R. aus Forschungs- und Entwicklungsabteilungen) der einzelnen Unternehmen. Abbildung 35 vermittelt einen Überblick der Institutionen, die das Projekt PrAllCon finanziell, materiell oder durch wissenschaftlichen Austausch unterstützten.

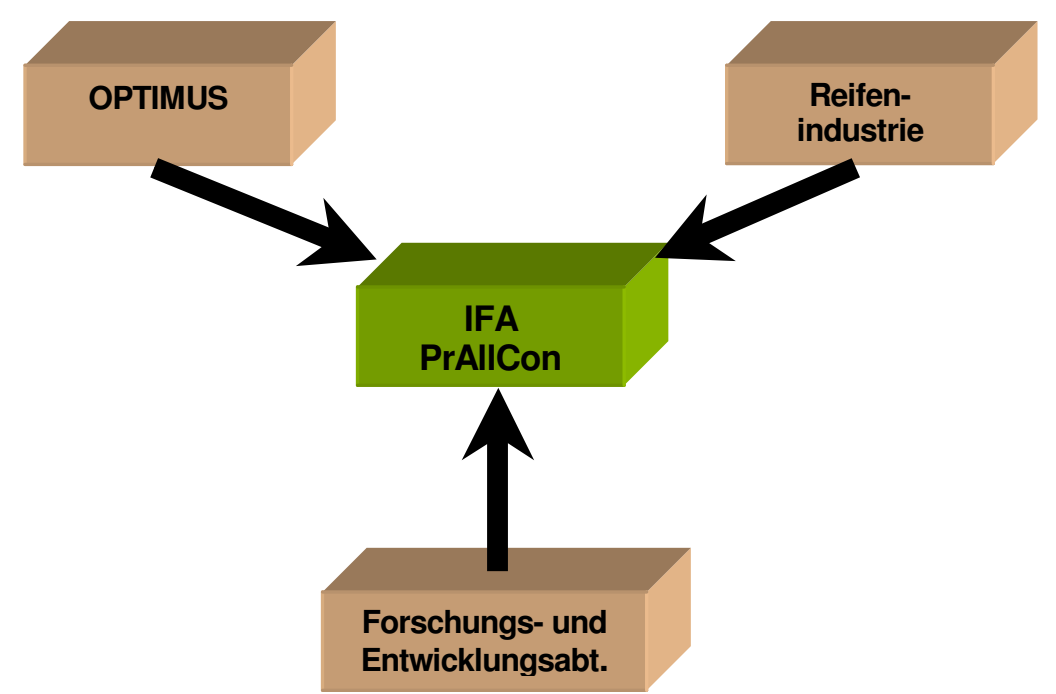

Abbildung 35: Institutionen, die finanziell, materiell oder durch wissenschaftlichen Austausch das Projekt PrAllCon unterstützten 


\section{Vorstudie}

\subsection{Hintergrund}

Mit der Vorstudie galt es die Messtechnik zu evaluieren und die Rahmenbedingungen für den Messablauf auf hartem und weichem Untergrund zu definieren. Sämtliche Komponenten der Messtechnik wurden hinsichtlich ihrer in dem Gesamtkomplex auszuführenden Funktionen überprüft. Dies bezog sich zum einen auf den reibungslosen Ablauf in technischer Hinsicht (Zusammenspiel aller Hardwarekomponenten des Radlastsimulators) und zum anderen auf die Integration der Messinstrumente (Kraftaufnehmer, I-Scan Software - Fa. Tekscan). Als Referenz für die Untersuchungen standen dem IFA zwei „Testreifen“ des Herstellers Nokian (600/55-26.5 12 PR TRS und 600/55-26.5 12 PR ELS) zur Verfügung.

\subsection{Messungen auf hartem Untergrund}

Zur Messung auf hartem Untergrund (s. Kap. 4.4) wurde der Reifen auf die in der Stahlplatte eingefassten Sensormatten gedrückt. Zum Schutz der Sensormatten wurde ein wachsbeschichtetes Papier verwendet, das die beim Anpressen des Reifens auf die Sensoroberfläche entstehenden Scherkräfte aufnahm und damit präventiv Beschädigungen verhinderte. Trotz des zusätzlichen Schutzes unterlagen die Sensormatten durch die starke Beanspruchung einem hohen Verschleiß.

Zu Beginn der Vorstudie wurde die Ausrichtung des Reifens bzw. des Reifenprofils bei der Ermittlung der Aufstandsfläche der Reifenstollen näher betrachtet. Hierzu wurde die Aufstandsfläche des Reifens bei einer Winkeldrehung von $30^{\circ}\left(12\right.$ Messungen $\left.\hat{=} 360^{\circ}\right)$ und einer Radlast von $30 \mathrm{kN}$ sowie einem Fülldruck von 3,5 bar untersucht. Die Ergebnisse zeigten, dass die Ausrichtungen des Reifens zu marginalen Abweichungen in den Aufstandsflächen führten. Wie in Tabelle 14 ersichtlich, variierte bei einer mittleren Aufstandsfläche von $555 \mathrm{~cm}^{2}$ und entsprechender Winkeldrehung von $30^{\circ}$ die Aufstandsfläche bei 12 Messungen im Mittel um ca. $3 \%$ bei einer Standardabweichung von 1,91 $\mathrm{cm}^{2}$.

Um diese geringen Abweichungen gänzlich auszuschließen und in der Arretierung des Reifens eine reproduzierbare und präzisere Erhebung der Daten zu erzielen, wurde bei den folgenden Messungen der Profilmittelpunkt des Reifens ausgemessen und zentriert auf den Messbereich ausgerichtet. 
Tabelle 14: Flächenermittlung bei einer Winkeldrehung von 30(NOKIAN 600/55-26.5 12 PR TRS, Radlast 30 kN, Fülldruck 3,5 bar)

\begin{tabular}{|c|c|c|c|c|c|c|}
\hline \multirow{2}{*}{ Grad [ [ ] } & \multicolumn{5}{|c|}{ Stollenaufstandsfläche $\left[\mathrm{cm}^{2}\right]$} & \multirow{2}{*}{$\begin{array}{c}\text { Abweichung der Stol- } \\
\text { lenaufstandsfläche zur } \\
\text { vorherigen Winkelum- } \\
\text { drehung [\%] }\end{array}$} \\
\hline & Matte 1 & Matte 2 & Matte 3 & Matte 4 & Gesamt & \\
\hline 30 & 152 & 140 & 100 & 180 & 572 & 1,06 \\
\hline 60 & 143 & 160 & 110 & 140 & 553 & 3,33 \\
\hline 90 & 150 & 144 & 145 & 105 & 544 & 1,63 \\
\hline 120 & 145 & 165 & 97 & 160 & 567 & 4,22 \\
\hline 150 & 127 & 147 & 180 & 105 & 559 & 1,42 \\
\hline 180 & 135 & 130 & 98 & 178 & 541 & 3,23 \\
\hline 210 & 126 & 163 & 150 & 120 & 559 & 3,33 \\
\hline 240 & 152 & 137 & 137 & 115 & 541 & 3,23 \\
\hline 270 & 145 & 154 & 114 & 135 & 548 & 1,28 \\
\hline 300 & 147 & 144 & 150 & 90 & 531 & 3,11 \\
\hline 330 & 144 & 146 & 102 & 184 & 576 & 8,47 \\
\hline $0=360$ & 142 & 150 & 176 & 98 & 566 & 1,74 \\
\hline Mittelwert & 142,33 & 148,33 & 129,92 & 134,17 & 554,75 & 3,15 \\
\hline Stdabw. & 8,37 & 10,18 & 29,07 & 32,58 & 13,44 & 1,91 \\
\hline Anzahl & 12 & 12 & 12 & 12 & 12 & 11,00 \\
\hline Minimum & 126 & 130 & 97 & 90 & 531 & 1,26 \\
\hline Maximum & 152 & 165 & 180 & 184 & 576 & 8,11 \\
\hline
\end{tabular}

Zur Erhebung der Messdaten wurden mehrere Kalibrierdateien erstellt und deren Verwendung innerhalb der Vorstudie überprüft. Die Bemessung der Reifen erfolgte nach den definierten Radlasten und Reifenfülldrücken (s. Kap. 4.4).

Der Vergleich der durch den Kraftaufnehmer und der über das Tekscan-System aufgenommenen Messwerte bestätigte eine Reproduzierbarkeit der aufgenommenen Daten mit geringen Differenzen. Die Fehlertoleranzen des Tekscan-Systems bewegten sich zwischen $+/-7$ bis $10 \%$ und entsprachen damit den Herstellerangaben.

Abbildung 36 zeigt einen Kontaktflächenabdruck des Nokian Reifens 600/55-26.5 12 PR ELS bei einer Laststufe von $30 \mathrm{kN}$ und einem Reifeninnendruck von 2,0 bar. Neben der graphischen Wiedergabe verbirgt sich hinter jeder Sensorzelle ein nummerischer Wert, der mit dem Kalibrierfile als Druckwert [bar] geladen wird. Ein exemplarischer Auszug einer Datenmatrix mit erhobenen Messwerten ist ebenfalls in der Abbildung 36 dargestellt.

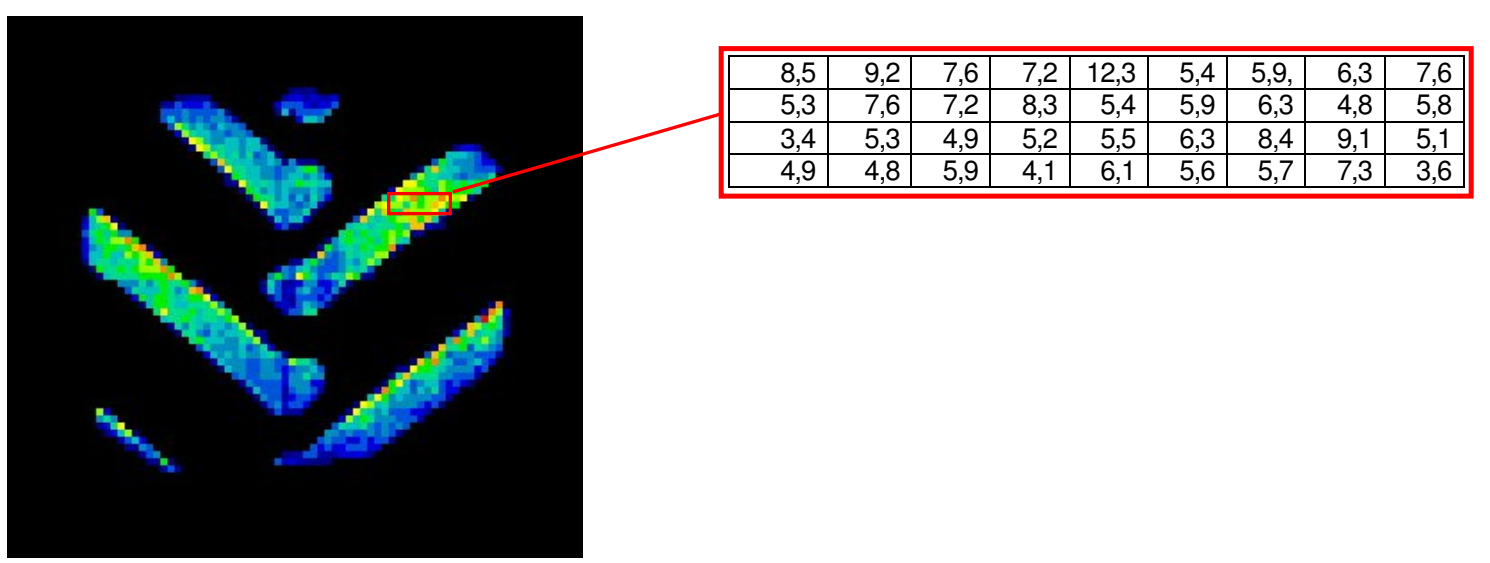

Abbildung 36: Kontaktflächendruckverteilung des Nokian Reifens 600/55-26.5 12 PR ELS bei einer Laststufe von $30 \mathrm{kN}$ und einem Reifeninnendruck von 2,0 bar, Auszug einer Datenmatrix - Messwerte geben die Druckverteilung in bar an 
Anhand der unterschiedlichen Farbabstufungen ist die Verteilung der Druckbelastung zu erkennen (der Druck nimmt ab über die Farben ROT / ORANGE / GELB / GRÜN / BLAU).

\subsection{Messungen auf weichem Untergrund}

Zur Bestimmung eines geeigneten Füllsubstrats wurden mehrere Materialien getestet. Im Einzelnen handelte es sich um Sand mit Korngrößen $<1 \mathrm{~mm}$, Grobsand-Feinkiesgemisch (1 - $4 \mathrm{~mm}$ ), Basaltsplitt (2 - $5 \mathrm{~mm}$ ), Kunststoffgranulat (2 - $3 \mathrm{~mm})$ und Polyesterschaum. Die Ergebnisse zeigten, dass der Sand mit der Korngröße $<1 \mathrm{~mm}$ die besten Resultate in Bezug auf die Wiedergabe der Druckverteilung in der Kontaktfläche erbrachte und die Stollenzwischenräume vollständig ausfüllte (Abb. 37). Die Textur besteht nach Angaben des Instituts für Bodenkunde und Waldernährung der Universität Göttingen, das mit einer Korngrößenanalyse beauftragt wurde, zu 99 \% aus Sand der Korngrößen 0,063 mm bis 0,63 mm und einem Prozent Schluff $(0,02 \mathrm{~mm}$ bis $0,063 \mathrm{~mm})$. Der Wassergehalt betrug zum Zeitpunkt der Analyse $4,5 \%$. Das Substrat lässt sich leicht aufschütten, durchharken und glatt abziehen (graden). Damit entsprach es den für die hohe Abfolge an standardisierten Messfolgen verlangten Anforderungen und garantierte deren Reproduzierbarkeit.

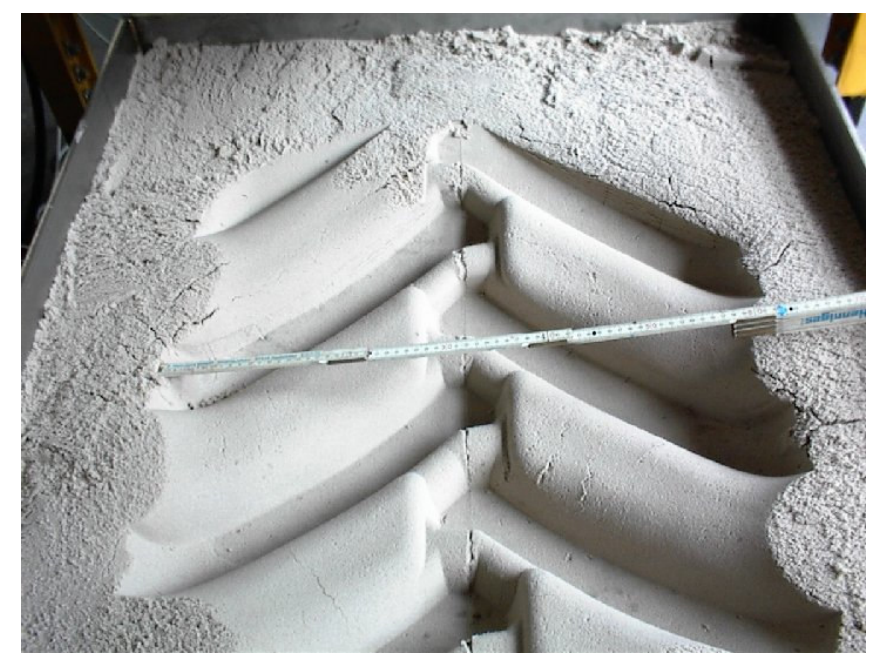

Abbildung 37: Reifenprofilabdruck mit Füllmaterial Sand der Korngröße $<1 \mathrm{~mm}$

Um ein Abzeichnen des Stollenprofils auf den Sensormatten bzw. deren Druckbildern zu vermeiden, musste eine ideale Füllhöhe definiert werden. Die in der Marktanalyse ermittelten Reifen und deren Profiltiefen dienten hierbei als Orientierung. Wie in Kapitel 3.4 angeführt, konnte analog zu den Profilarten ebenfalls eine Klassifikation der Profiltiefen auf dieser Basis erfolgen. So weisen Reifen mit Traktionsprofilen i. d. R. Tiefen von 45 - 55 mm und Reifen mit Softprofilen Tiefen von 30 - 44 mm auf. Die dem IFA zur Verfügung gestellten Referenzreifen bewegten sich mit Profiltiefen von $53 \mathrm{~mm}$ und $30 \mathrm{~mm}$ im jeweils oberen bzw. unteren Bereich dieser Vorgaben. Beginnend mit einer Füllhöhe von $10 \mathrm{~cm}$ wurde diese sukzessive in $5 \mathrm{~cm}$ Abständen bis $25 \mathrm{~cm}$ erhöht und die Kontaktflächenabdrucke ausgewertet. Eine Höhe von $20 \mathrm{~cm}$ erfüllte alle Voraussetzungen, die Drücke in der Kontaktfläche - ohne Abbildung des Stollenprofils - sowohl für Reifen mit Traktions- als auch mit Softprofilen sichtbar werden zu lassen. 
Der Verfahrensablauf der Messungen sah ein Entnehmen des Sandes nach Abschluss eines Messtages vor, da sich das Verbleiben der Last des Sandes über Nacht negativ auf die Sensormatten, respektive die Messergebnisse, auswirkte. Die Sensormatten wurden vor Aufschüttung des Sandes mit vier Lagen Wachspapier und einer $3 \mathrm{~mm}$ dicken Silikonfolie bedeckt, um direkte Beschädigung zu vermeiden.

Für die Untersuchungen auf weichem Untergrund mussten eigene Kalibrierdateien erstellt werden. Ansonsten entsprachen die Messungen dem in Kapitel 4.4 beschriebenen Ablauf. Die Ergebnisse der aufgenommenen Daten (Kraftaufnehmer, Tekscan-System) bestätigten ebenfalls eine Reproduzierbarkeit und im Vergleich zu den Messwerten auf hartem Untergrund, wiesen diese eine höhere Genauigkeit auf. 


\section{Hauptstudie}

\subsection{Datenaufnahme}

Die eigentliche Bemessung der Reifen erfolgte von April 2003 bis Mai 2004. Die Bedienung der Messanlagen erforderte erhebliches Personal: die Bemessung eines Reifens wurde in der Vorplanung mit 2,5 Manntagen veranschlagt und konnte im Verlauf der Datenaufnahme bestätigt werden.

Das in Abbildung 38 dargestellte Flussdiagramm vermittelt einen Überblick über die im Einzelnen durchgeführten Untersuchungen.

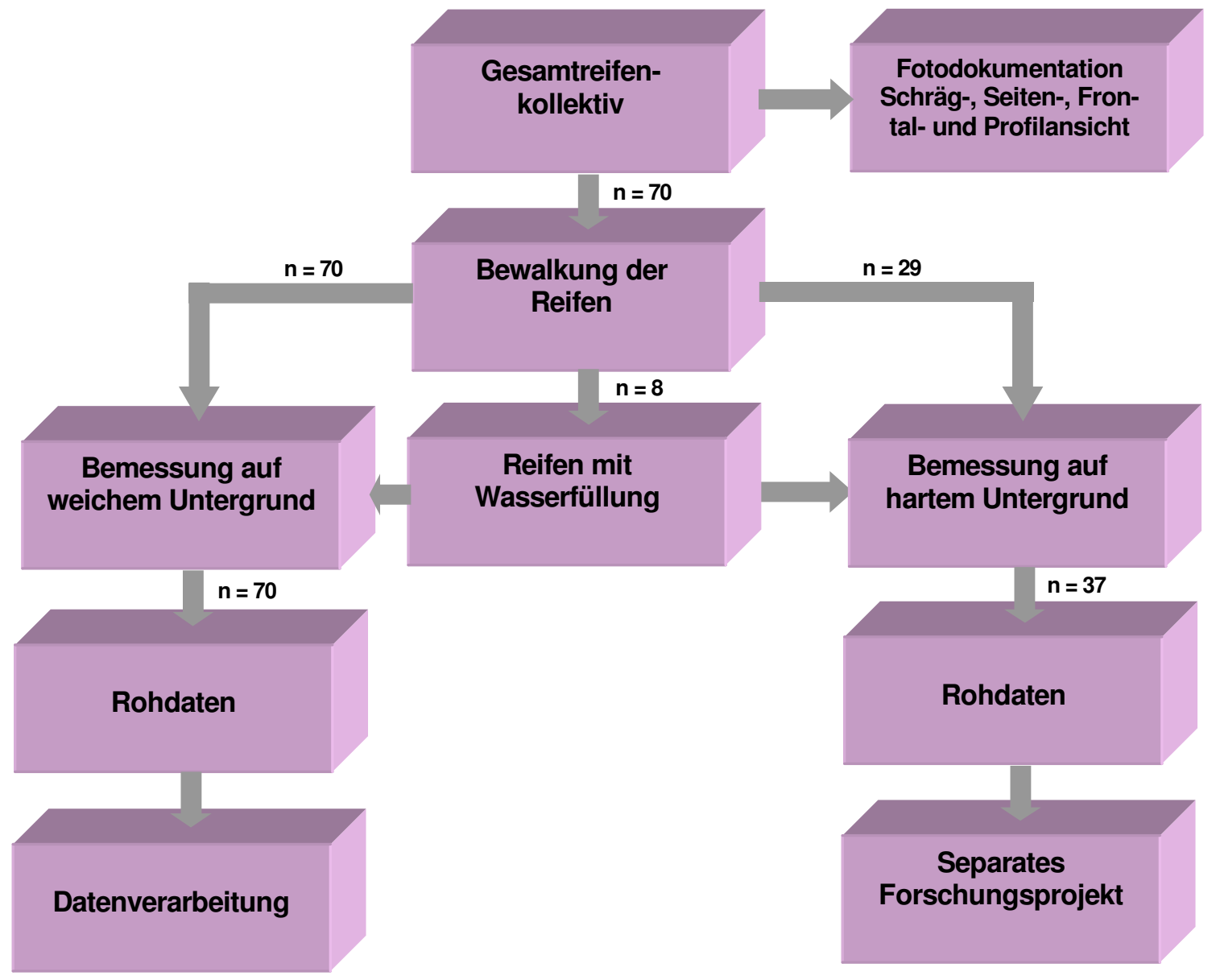

Abbildung 38: Flussdiagramm Datenaufnahme

Wie aus dem Flussdiagramm zu entnehmen, gliedert sich dieses in einen linken und rechten Zweig. Wird der linke Zweig verfolgt, so ist zu erkennen, dass das gesamte Reifenkollektiv von 70 Reifen auf weichem Untergrund bemessen wurde. Bei diesen erhobenen Kontaktflächenabdrucken wurden nicht nur die Stollen, sondern auch die Stollenzwischenräume berücksichtigt, die beim Einsinken des Reifens in den Waldboden ebenso tragende Funktionen übernehmen. Auf diese als Ganzes zu erfassenden Kontaktflächenabdrucke beschränken sich die weiteren Ausführungen, insbesondere die detaillierte Vorstellung der Erhebung und Auswertung der Daten. 
Der rechte Zweig zeigt die Abfolge der Messungen auf hartem Untergrund, deren Anzahl insgesamt 29 Reifen beträgt. Dieses separate Forschungsprojekt wurde parallel zu den Messungen auf weichem Untergrund durchgeführt. Der zusätzliche Messaufwand und zeitliche Restriktionen machten es erforderlich, dass Reifenkollektiv zu begrenzen. Es wurden Reifen berücksichtigt, die auf Grundlage der Marktanalyse besonders häufig auf Maschinen zu finden sind, ergänzt um einige Neuentwicklungen, von denen zu erwarten ist, dass sie bereits verwendete Reifendimensionen in Zukunft ersetzen werden oder bereits ersetzen. Zudem wurde darauf geachtet, dass die ausgewählten Reifendimensionen hinsichtlich ihrer charakterisierenden Parameter, wie z. B. Nennbreite und Durchmesser, ein ausgewogenes Verhältnis aufweisen.

Die Befüllung von Reifen mit schwerpunktverlagernder Flüssigkeit ist sehr zeitaufwendig, wurde aber dennoch an acht für solche Gepflogenheit charakteristischen Rädern vorgenommen. Bei diesen acht Rädern kann davon ausgegangen werden, dass sie nahezu alle in der Praxis mit Flüssigkeit befüllten Reifen abdecken (MÜLLER, 2003; FENSEL, 2003). Mit Rücksichtnahme auf die Belange der Reifenhersteller und deren Bestreben, die Reifen nach den Messungen wieder zu veräußern, wurde bei deren Befüllung nur auf Wasser zurückgegriffen und auf die Zugabe von Gefrierinhibitoren (Calciumchlorid/Magnesiumchlorid/Glykol) verzichtet. Die Bemessung dieser Reifen erfolgte sowohl auf weichem als auch auf hartem Untergrund. Eine weitere Auswertung bzw. vergleichende Bewertung mit den nur luftgefüllten Reifen gleichen Typs erfolgt in dieser Arbeit nicht.

Bei fünf definierten Radlasten und sieben Fülldrücken wurden pro Reifen 35 Kontaktflächendruckverteilungen produziert. Das bedeutete bei einer Anzahl von 70 Reifen 2.450, bei 29 Reifen 1.015 und bei jeweils 8 Reifen 280 Kontaktflächendruckverteilungen. Demzufolge lag für jeden Kontaktflächenabdruck nur eine Messung vor. Mehrere Reifen gleichen Typs standen nicht zur Verfügung, so dass kein Vergleich von mehreren Kontaktflächenabdrucken durchgeführt wurde. Insgesamt konnten 2.445 Datensätze in der Auswertung für die Messungen auf weichem Untergrund berücksichtigt werden. Die Minderung des Stichprobenumfangs lag darin begründet, dass sich die Reifen M01, T01, T03 und T13 bei Laststufen von 40 kN / 50 kN und einem Fülldruck von 0,5 bar über die Abmessungen der Sandbox ausdehnten und sich somit keine Kontaktflächenabdrucke erfassen ließen.

Eine vollständige Übersicht der bemessenen Reifen befindet sich nach Herstellern gegliedert im Anhang 3 in den Tabellen 3.1 bis 3.6. Die einzelnen Reifenkollektive wurden dabei in Standard- und Niederquerschnittreifen unterteilt und nach Nennbreite und Felgendurchmesser sortiert.

Die für jeden Reifen auf weichem Untergrund erhobenen Kontaktflächendruckverteilungen (inklusive der mit Wasserfüllung bemessenen Reifen) und die fotografische Dokumentation der Schräg-, Seiten-, Frontal- und Profilansicht, die eine Vorstellung der einzelnen Reifendimensionen vermitteln, sind in einem separaten Anhang (6) in Form einer PDF-Datei auf CD im Buchdeckel zu finden. Exemplarisch ist für den Reifen Trelleborg 600/55-26.5 428 (T20) diese Art der Darstellung im Anhang 4 angeführt.

Eine erste nähere Betrachtung der hier relevanten Messungen auf weichem Untergrund zeigt, dass die Ausprägung des Kontaktflächendruckes in Abhängigkeit der Radlasten und der Fülldrücke nicht homogen verteilt ist. Es treten Differenzierungen in Form von isobaren Zonen auf. 
Damit wird die im Kapitel 2.1 formulierte Hypothese über die Kontaktflächendruckverteilung manifestiert.

Generell konnte festgestellt werden, dass sich mit zunehmender Radlast und steigendem Fülldruck der Schwerpunkt der Druckbelastung in der Mitte der Kontaktfläche konzentriert und stetig zur Peripherie hin abnimmt. Anhand der unterschiedlichen Farbabstufungen (der Druck nimmt ab über die Farben ROT / GELB / GRÜN / BLAU) ist die Verteilung der Druckbelastung der Kontaktflächen deutlich zu erkennen. Dieser Prozess soll exemplarisch an drei Kontaktflächenabdrucken - bei einer konstanten Radlast von $30 \mathrm{kN}$ und variierenden Fülldrücken von 1,0 bar, 3,0 bar und 5,0 bar - dargestellt werden. Ist bei einem Fülldruck von 1,0 bar die Kontaktflächendruckverteilung noch wenig differenziert, findet mit Zunahme des Reifeninnendruckes eine erkennbare Verschiebung der Druckbelastung in Richtung Reifenmittelpunkt statt (Abb. 39). Die abgebildeten Druckverteilungszonen sind dabei vergleichbar mit einem horizontalen Schnitt durch eine Druckzwiebel ${ }^{20}$.
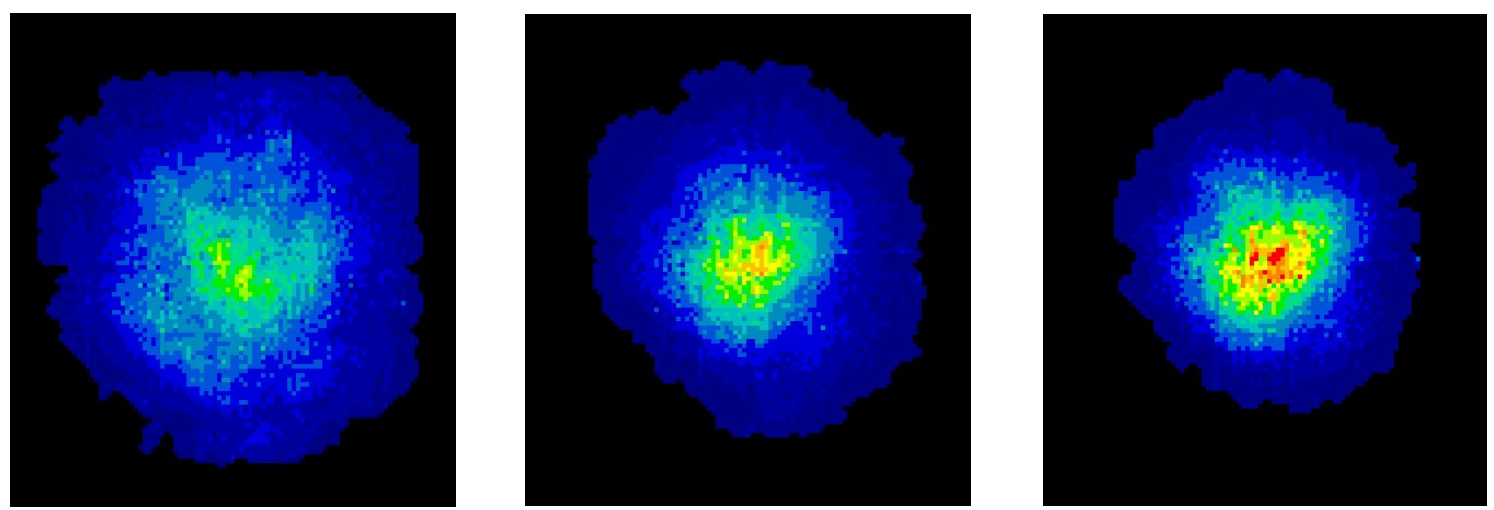

Abbildung 39: Darstellung der Druckbelastung bei konstant gehaltener Radlast von $30 \mathrm{kN}$ und variierenden Fülldrücken (v. I. n. r. 1,0 bar, 3,0 bar, 5,0 bar). Anhand der unterschiedlichen Farbabstufungen (der Druck nimmt ab über die Farben ROT / GELB / GRÜN / BLAU) ist die Verteilung der Druckbelastung zu erkennen.

Bevor die als Rohdaten erhobenen Kontaktflächenabdrucke einer statistischen Analyse unterzogen werden konnten, mussten sie entsprechend präpariert werden. Die weitere Verarbeitung der Daten wird in dem nachfolgend aufgeführten Kapitel 6.2 beschrieben.

Es sei hier abschließend erwähnt, dass neben der eigentlichen Erhebung von Daten weitere unerlässliche Arbeiten hinzukamen, die einerseits zur Gewährleistung eines einwandfreien Messablaufs notwendig und andererseits für eine zusätzliche Datengewinnung erforderlich waren. Hierzu zählten u. a. das regelmäßige Equi- und Kalibrieren der Sensormatten, das Ausrichten/Zentrieren des Reifens und, sofern erforderlich, Überstandsmessungen bei Überschreitung des regulären Messbereiches der Sensormatten.

20 Würden die Punkte gleichen Druckes am Querschnitt eines Bodenprofils aufgezeichnet, so ergäben sich Ringe, die vom Zentrum der Auflast ausgehend in immer größeren Abstand auftreten, wie die Schalen einer Zwiebel (ERLER, 2000). 


\subsection{Datenverarbeitung}

Die I-Scan-Software (Tekscan, Version 5.01) kann für die Betriebssysteme DOS, WIN95/98 und WIN-NT eingesetzt werden und ist in Anlehnung der Windowsoberfläche von Microsoft menügesteuert eingerichtet. Durch eine ISA-PC Karte werden die von den Sensormatten detektierten Druckänderungen als Bild aufgezeichnet und eingelesen. Die Darstellung der Kontaktflächenabdrucke ist in zwei- oder dreidimensionalen Bildern möglich. Zudem können die aufgenommenen Daten parallel zur Messung, aber auch unabhängig davon in unterschiedlichen Darstellungsvarianten bearbeitet werden. Weiterhin erlaubt eine Datenkonvertierung nach ASCII die Analyse und Weiterverarbeitung mit anderen Softwareprogrammen.

Um ein Gesamtbild des Reifenabdruckes zu bekommen, wurden die Daten jeder einzelnen Sensormatte eingelesen und im Tekscan eigenen FSX-Format abgespeichert. In der Abbildung 40 ist die Kontaktflächendruckverteilung auf jeder einzelnen der insgesamt vier TekscanSensormatten zu erkennen, die zu einem Gesamtbild zusammengesetzt werden. Damit stellten diese Dateien die Rohdatenbasis dar. Nach jeweils zehn bemessenen Reifen wurden neue Equilibrier- und Kalibrierdateien mit der I-Scan-Software erstellt. Bei dem zu bemessenden Kollektiv von 70 Reifen entspricht dies vierzehn solcher Dateien. Jede einzelne Rohdatei musste mit der jeweiligen Equilibrier- und Kalibrierdatei geladen werden. Im Anschluss daran erfolgte eine Konvertierung der Daten nach ASCII.

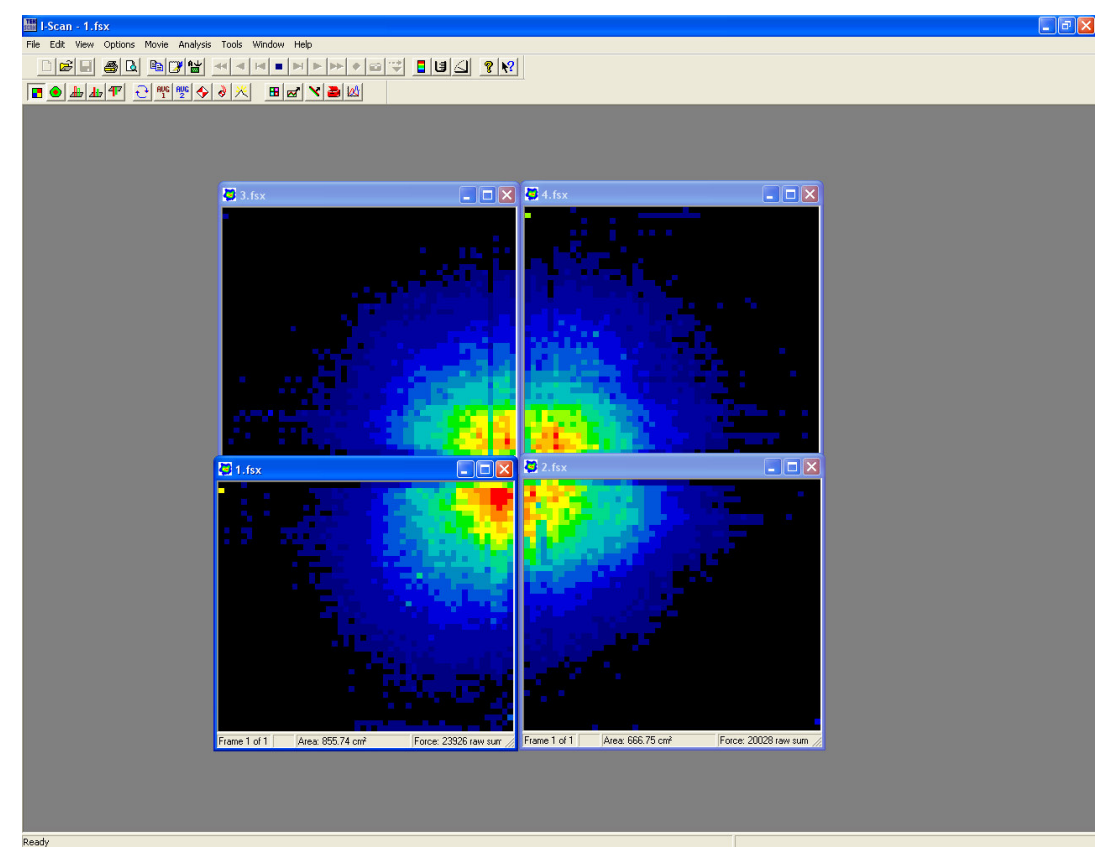

Abbildung 40: Benutzeroberfläche der I-Scan-Sofware (Tekscan, Version 5.01)

Die weitere Verarbeitung und Analyse der Messdaten fand mit am IFA entwickelten Programmen (Programmiersprache C) in Kombination mit wissenschaftlich etablierter Standardsoftware, wie z. B. SPSS, statt. Die einzelnen Schritte der Datenverarbeitung und die hierzu verwendeten Programme zeigt folgendes Flussdiagramm (Abb. 41). 


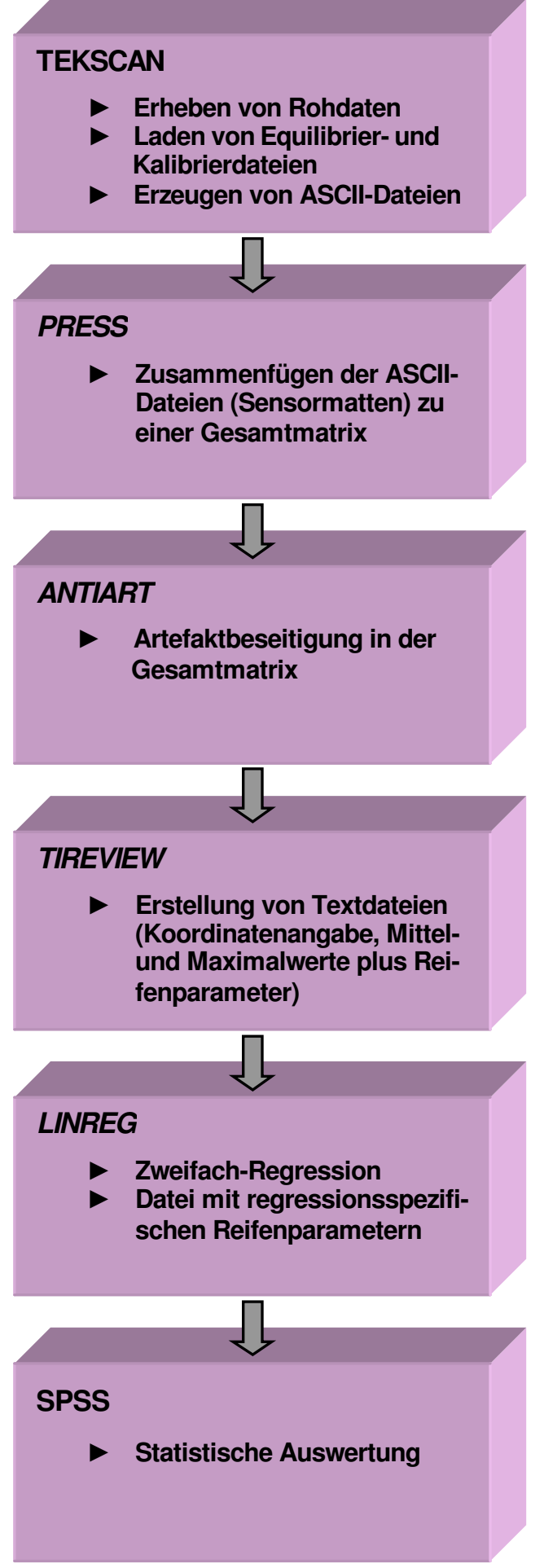

Abbildung 41: Flussdiagramm Datenverarbeitung (alle kursiv geschriebenen Programme sind Eigenentwicklungen des IFA)

Die erzeugten ASCII-Dateien wurden mit einem Programm namens PRESS, das die Daten jeder einzelnen Sensormatte (vier bzw. acht bei Überstandsmessungen) zu einer Gesamtdatenmatrix zusammenfügt, verarbeitet. Messtechnische Ungenauigkeiten, insbesondere eine Übersteuerung einzelner Sensorzellen und/oder der partielle Ausfall von Sensorreihen und -spalten, die während der starken Beanspruchung der Sensormatten auftraten, machten eine Bearbeitung der Kontaktflächenabdrucke mittels verschiedener Artefaktroutinen erforderlich (Abb. 42). 

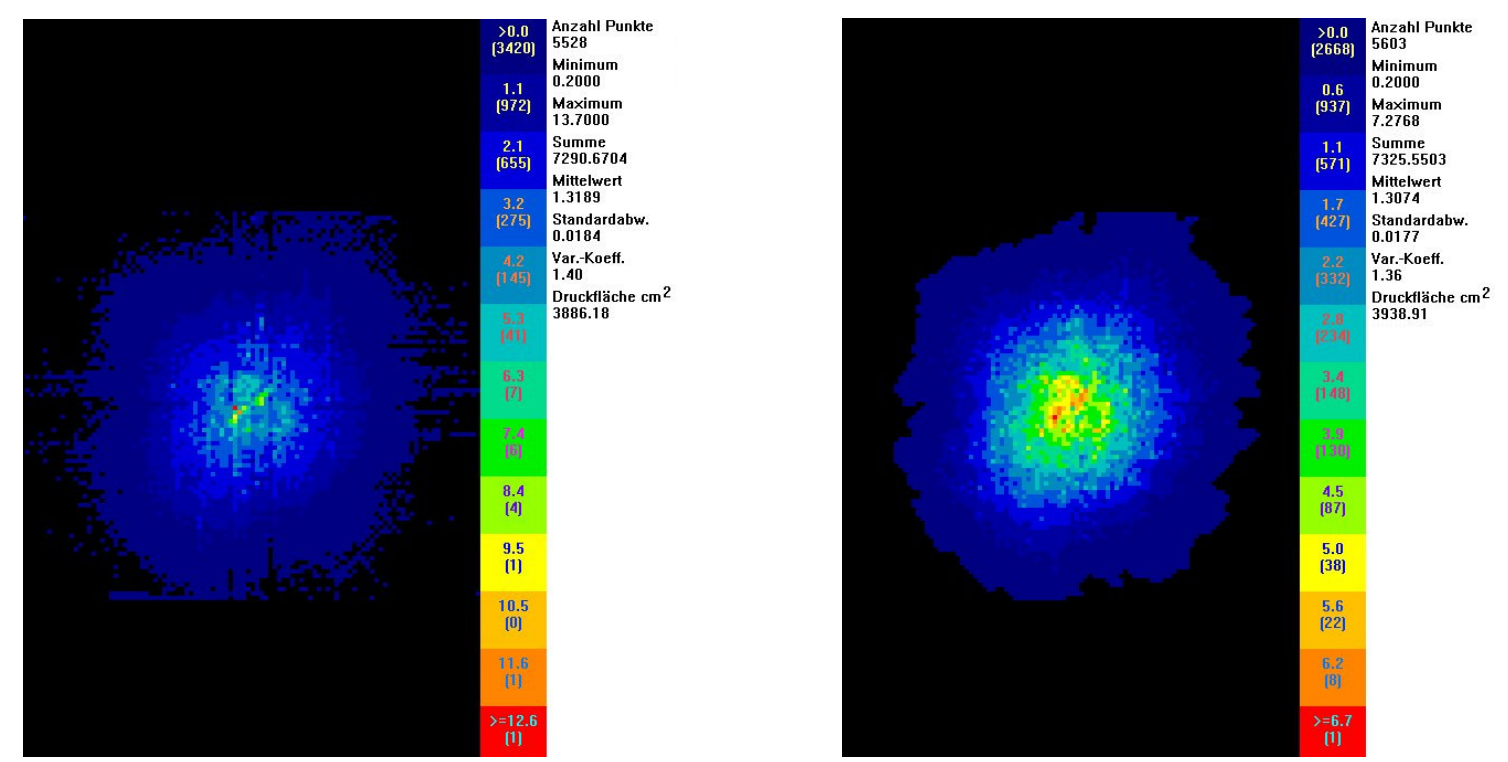

Abbildung 42: Artefakteliminierung am Beispiel einer Kontaktflächendruckverteilung (Radlast 50 kN, Fülldruck 5,0 bar) - der Häufigkeitstabelle am linken Bildrand sind Druckwerte zu entnehmen (links: ohne Ausreißerbeseitigung, rechts: nach Beseitigung von Artefakten)

Eine entsprechende Behandlung der Artefakte erfolgte mit dem Programm ANTIART. Dieses Programm bietet die Möglichkeit einer Mittelung aller Pixel, deren Werte über einem bestimmten Grenzwert liegen, in Prozent vom Mittelwert oder absolut. Des Weiteren können Pixel mit 1,2,3 oder 4 Nachbarn eliminiert und ausgefallene Messzellen/Pixel innerhalb der Reifenaufstandsfläche durch Mitteln von mehr als 4,5,6 und 7 Nachbarn ergänzt werden. Hiermit wurde eine Abrundung des Kontaktflächenabdruckes in seinem Randbereich erzielt. Ausgefallene Reihen konnten zudem manuell durch horizontales und vertikales Mitteln im Kontaktflächenabdruck geschlossen werden. Eine gezielte Löschung von Ausreißern durch exaktes Ansteuern per Fadenkreuz war ebenfalls möglich.

Die bereinigten ASCII-Dateien wurden in einem nächsten Schritt durch das Programm TIREVIEW eingelesen. Dieses Programm bietet neben einer graphischen Darstellung sämtlicher Kontaktflächenabdrucke eines Reifens, auch die Alternative, die graphischen Verläufe der Mittel- oder Maximalwerte in Richtung der Abszissen- und Ordinatenachse wiederzugeben. TI$R E V I E W$ ist zudem in der Lage, die für eine weitere Auswertung der Daten unerlässliche Erstellung von Textdateien, die Koordinatenangaben, eingelesene Mittel- und Maximalwerte sowie Reifenparameter beinhalten, zu erzeugen. Einzelne, den Reifen kennzeichnende Parameter können separat angezeigt und somit kurzfristig abgerufen werden.

Die erzeugten Textdateien waren die Grundlage einer ersten statistischen Analyse, die mittels des Programms LINREG ausgeführt wurde. Dieses Programm rechnet eine lineare ZweifachRegression und erzeugt Dateien, aus denen Regressionsparameter entnommen und im Standardprogramm SPSS statistisch ausgewertet wurden. 


\section{Datenauswertung und statistische Methoden}

\subsection{Interpretation der Druckverteilung}

Eine dreidimensionale Darstellung der Kontaktflächenabdrucke lässt erkennen, dass der Druck sich durchweg in Form einer Glocke mit elliptischer Querschnittsfläche verteilt. Eine exemplarische Illustration einer solchen „Druckverteilungsglocke“ für den Reifen T22 (750/45-30.5 169/A8 428) bei einer Radlast von 30 kN und einem Fülldruck von 3,0 bar zeigt Abbildung 43. Vertikalschnitte sowohl in Fahrtrichtung als auch in der Breitenausprägung der Reifen sind demzufolge ebenfalls typische Glockenkurven.

Die "Verteilungsglocken" entsprechen einer statischen Momentaufnahme beim zweckkonformen Einsatz eines solchen Reifens zum Antrieb einer Arbeitsmaschine. Eine „breite Ausprägung" einer solchen Glocke bei relativ hohen Drücken ist gleichbedeutend mit einer ceteris paribus hohen Traktionsmöglichkeit des Reifens, wobei klar ist, dass diese Traktion auch wesentlich über die Profilierung umgesetzt wird. Diese Glockenausprägung informiert demnach über eine mechanisch-technische Eigenschaft des Reifens.

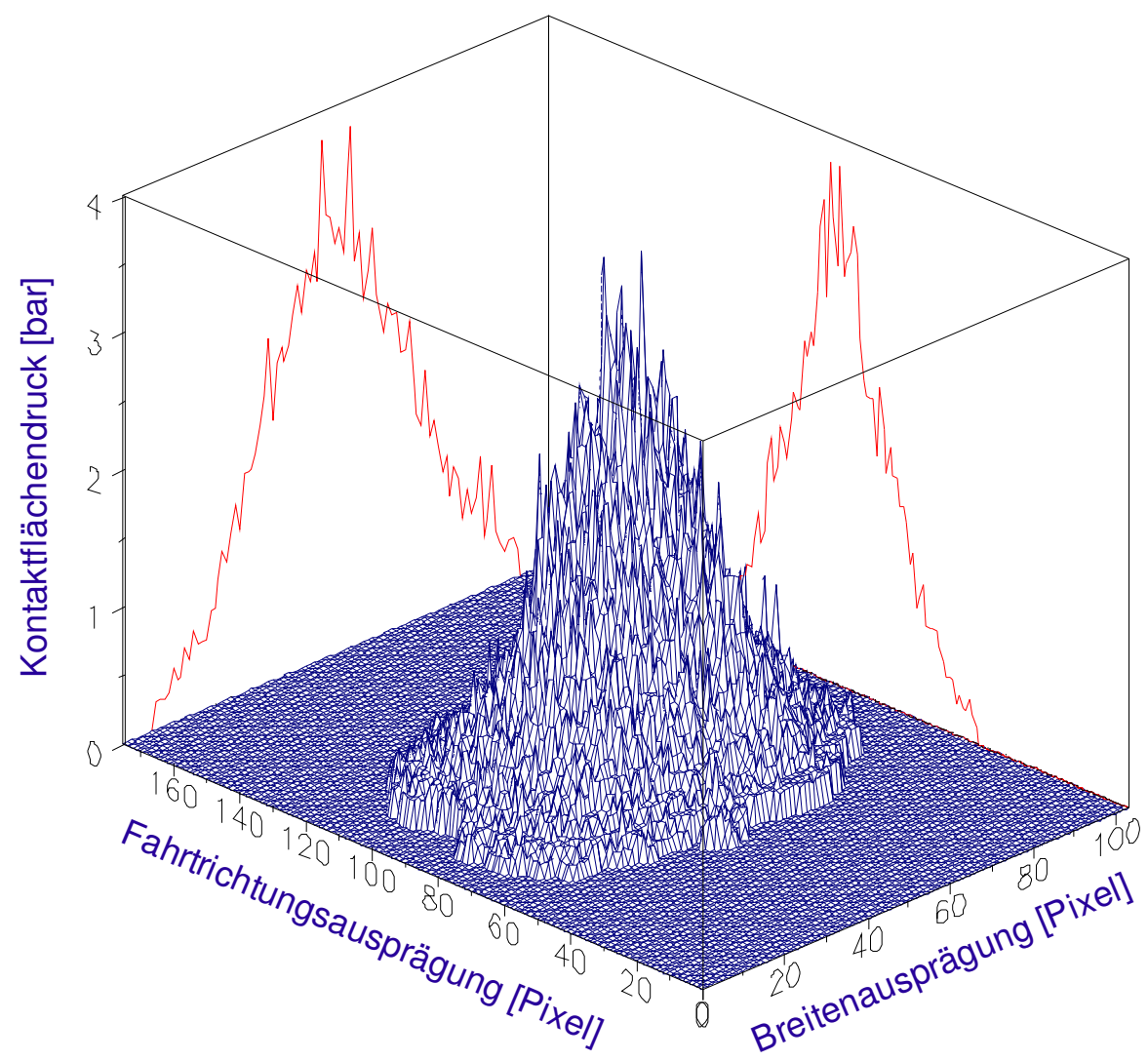

Abbildung 43: Dreidimensionale Darstellung einer Kontaktflächendruckverteilung (Reifen T22, Radlast 30 kN, Fülldruck 3,0 bar)

Zur Würdigung des bodenökologischen Aspekts muss man das Bild der Glocke auf die Arbeitssituation der angetriebenen Maschine abstrahieren, mit anderen Worten den dynamischen Einsatz in erster Näherung simulieren: es ergibt sich für die bemessene Situation 
(20 cm unter dem definierten Sand) eine „Druckrinne“ mit einem Querschnitt, der der maximalen Ausprägung der (statischen) Glocke in der Reifenbreite entspricht (Abb. 44). Ausschlaggebend ist also die Maximalverteilung des Druckes in der Breitenausprägung.

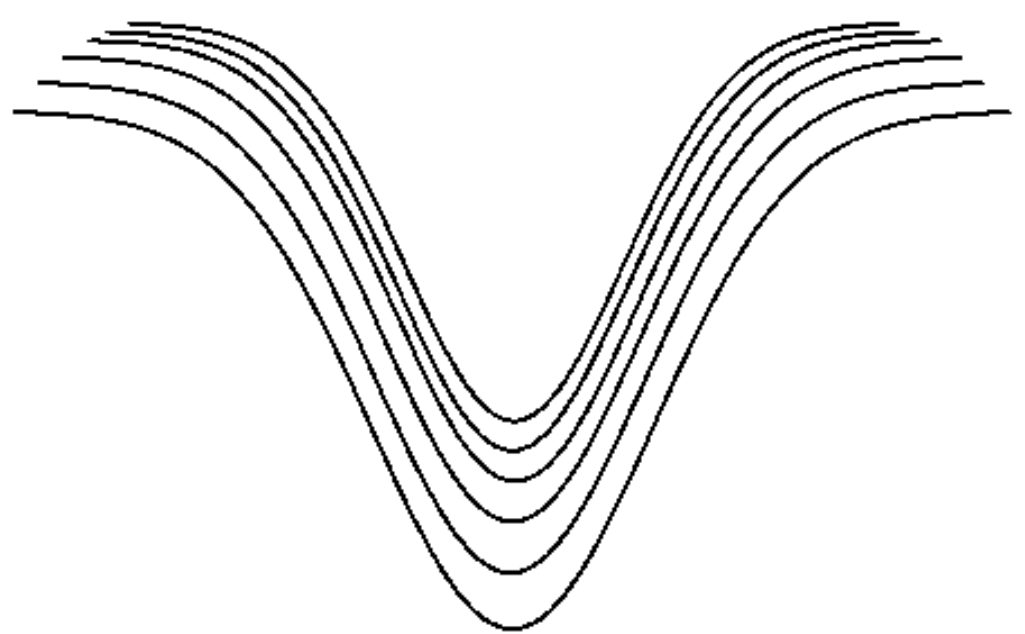

Abbildung 44: Maximalverteilung des Druckes in Breitenausprägung (Querschnittsdarstellung „Druckrinne“)

\subsection{Auswertungsansätze}

Die Auswertung der umfangreichen Datenmatrizen der einzelnen Kontaktflächendruckverteilungen bedurfte eines Verfahrens, welches alle Reifen erfasst und einen Vergleich dieser ermöglicht. Hierzu galt es zu überprüfen, wie eine potentielle Auswertung der aufbereiteten Daten zu erfolgen hat.

Um die auftretenden Drücke zu erfassen, erschien eine Auswertung der Messwerte in der Breiten- und Fahrtrichtungsausprägung am geeignetesten. Bei diesen in Breite und Fahrtrichtung vorgenommenen Vertikalschnitten durch die Verteilungsglocken werden die maximalen Messwerte jeder einzelnen Zeile und Spalte ermittelt.

Ausgangspunkt der Berechnungen sind die vier zusammengefügten Sensormatten und ihre Messzellen, die in Breiten- und Fahrtrichtung eine Matrix bilden. Jede Sensorzelle stellt hierbei einen Pixel dar. Der Abstand einer Sensorzelle beträgt in Breiten- und in Fahrtrichtung $0,00838 \mathrm{~m}$. In Breitenrichtung stehen $\mathrm{x}_{0}, \mathrm{x}_{1}, \mathrm{x}_{2} . . \mathrm{x}_{103}$ und in Fahrtrichtung $\mathrm{y}_{0}, \mathrm{y}_{1}, \mathrm{y}_{2} . . \mathrm{y}_{175}$ Pixel $^{21}$ zur Verfügung (Abb. 43). Diese Abstände bilden einerseits die Basis die Kontaktflächendrücke zu lokalisieren und andererseits ihre absoluten Ausprägungen in Breiten- und Fahrtrichtung zu erfassen.

Innerhalb jeder Spalte und Zeile werden alle gemessenen Einzelwerte miteinander verglichen und der maximale Druckwert ausgegeben. Nicht belastete Pixel (0-Werte) werden bei diesen Berechnungen ausgeschlossen. Auf Grundlage dieser Berechnungen geben die einzeln abgetragenen Maximalwerte den Verlauf des jeweiligen Kontaktflächendruckes in Breiten- und

\footnotetext{
${ }^{21}$ Kontaktflächen von Reifen, die sich in Fahrtrichtung über den standardisierten Messbereich der Sensormatten hinaus ausdehnten, wurden durch die Verschiebung des Messtisches erfasst (s. Kap. 4.4). Die Anzahl der pro Zeile zur Verfügung stehenden Pixel erhöhte sich von 88 auf 176.
} 
Fahrtrichtung wieder, wie sie in den Schattenrissen ${ }^{22}$ in Abbildung 43 zu erkennen sind. In Abbildung 45 ist exemplarisch der Druckverlauf in Breitenrichtung für den Reifen T22 (750/4530.5 169/A8 428) bei einer Radlast von 30 kN und einem Fülldruck von 3,0 bar dargestellt.

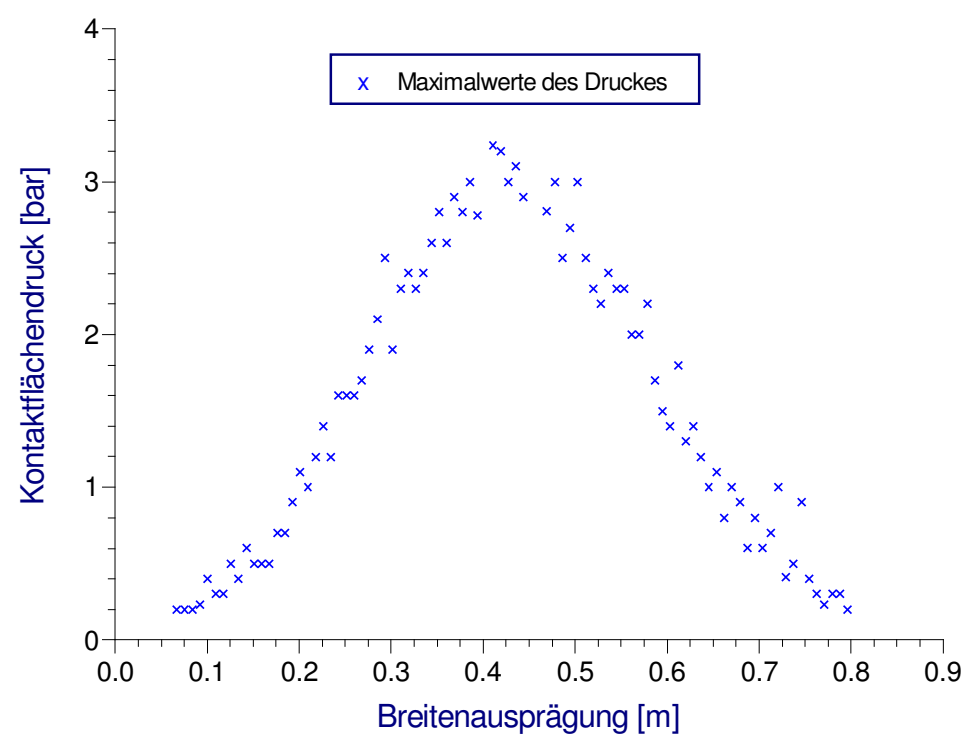

Abbildung 45: Verlauf des maximalen Kontaktflächendruckes in Breitenrichtung (Reifen T22, Radlast 30 kN, Fülldruck 3,0 bar)

Die Auswertungen zeigten, dass sich die Druckwerte in Breiten- und Fahrtrichtung exponentiell darstellen und in ihrer Verteilung einer Glockenkurve ähneln. Ziel einer Berechnung war es deshalb, für die Druckverteilungen aller Reifen eine möglichst gute Kurvenanpassung zu erhalten.

\subsection{Quantifizierung der Druckverteilung}

Eine Literaturrecherche sollte helfen, eine Funktion für den Ausgleich der Druckwerte zu finden. Als mögliche Ausgleichsfunktion für jegliche Druckverteilung bot sich die in Gleichung 2 dargestellte Funktion an (BRONSTEIN ET AL, 2001).

$$
y=a \cdot e^{b \cdot x+c \cdot x^{2}}
$$

Die Kurve ist symmetrisch zur vertikalen Achse

$$
x=-\frac{b}{2 \cdot c}
$$

\footnotetext{
22 Darstellung der höchsten Druckwerte in Breiten- und Fahrtrichtungsausprägung, die sich ergeben, wenn man parallele
} Lichtstrahlen quer und längs zum aufgedrückten Reifen über das Druckprofil auf eine imaginäre Leinwand projeziert. 
Sie schmiegt sich im Falle von $c<0$ für kleine und große $x$ der Abszisse an und wölbt sich positiv bei $a>0$.

Die Wendepunkte ${ }^{23}$ haben in diesem Falle die Koordinaten

$$
\frac{-b \pm \sqrt{-2 \cdot c}}{2 \cdot c}, a \cdot e^{\frac{-\left(b^{2}+2 \cdot c\right)}{4 \cdot c}}
$$

Bei der vorliegenden Ausgleichsfunktion handelt es sich um eine Exponentialfunktion zur Basis e, der Eulerschen Zahl ( 2 2,71828). Der Zusammenhang zwischen der abhängigen Variablen (Regressand) y und der erklärenden oder unabhängigen Variablen (Regressor) x kann in dieser Form nicht linear-regressiv ausgeglichen werden. Wenn man (1) jedoch beidseitig logarithmiert, wird daraus:

$$
\ln y=\ln a+b \cdot x+c \cdot x^{2}
$$

Der regressive Ausgleich einer entsprechenden Punkteschar erfolgt über einen multiplen Linearansatz, also nach Logarithmierung (zur Basis e) der Zielgröße y und Ergänzung des quadrierten Regressors x. Diese Funktionsgleichung ist in der Lage, einen linearen Zusammenhang zwischen dem Logarithmus der abhängigen Variablen y (Kontaktflächendruck) und der unveränderten erklärenden Variablen $x$ und $x^{2} z u$ beschreiben. Bei den erklärenden Variablen handelt es sich um die Koordinaten der Breitenausprägung, deren Skalierung in Meter angegeben ist. Für den vorliegenden Fall ist es somit möglich, den Zusammenhang zwischen den logarithmierten Werten von y und den $\mathrm{x}$-Werten in einer linearen Zweifachregression zu untersuchen. Nach späterer Delogarithmierung wird aus (5) wiederum (2). Somit erhält man die gesuchten Konstanten a, b und c der Originalgleichung (2).

Wie dem Kapitel 6.2 zu entnehmen, wurde die lineare Zweifachregression mittels des Programms LINREG gerechnet. Aus den Textdateien werden die Variablen Druck [bar] und Pixelpositionen eingelesen. Die Pixelpositionen werden in absolute Meterangaben umgerechnet und zudem eine neue Variable als Quadrat der Position in Meter erzeugt. Die lineare Zweifachregression wird mit der logarithmierten Zielgröße „Druck“[bar] und den unabhängigen Variablen „Position“ [m] und dem „Quadrat der Position“ $\left[\mathrm{m}^{2}\right]$ durchgeführt. Als Ergebnis erzeugt LIN$R E G$ in der logarithmierten Phase eine Datei, die fülldruck- und lastspezifisch die Ergebnisse der Zweifachregression (z. B. Regressionskoeffizienten ${ }^{24}$ und Bestimmtheitsmaße ${ }^{25}$ ) sowie weitere relevante Parameter (z. B. Gültigkeitsbereich der jeweiligen Funktionsgleichung) enthält.

\footnotetext{
23 Als Wendepunkte einer Funktion werden die Stellen bezeichnet, an denen die Kurve von einer Linkskrümmung in eine Rechtskrümmung übergeht oder umgekehrt. Die zweite Ableitung muss in diesem Punkt gleich Null sein.

24 Steigungsmaß der unabhängigen Variablen in der Regressionsgleichung.

25 Das Bestimmtheitsmaß misst den Anteil der erklärten an der gesamten Streuung der Zielgröße.
} 
Für den Ausgleich in Fahrtrichtung ist die Berechnung analog zu der eben beschriebenen, mit dem einen Unterschied, dass die einbezogenen Variablen nicht aus den Spalten, sondern aus den Zeilen der Datenmatrix gewonnen werden.

Abbildung 46 zeigt den Ausgleich des maximalen Druckes in der Breitenausprägung nach dem beschriebenen Modell für den bereits oben erwähnten Reifen T22 bei einer Radlast von $30 \mathrm{kN}$ und einem Fülldruck von 3,0 bar. Die Voraussetzungen und Konsequenzen treffen für den ausgeglichenen Bereich des steigenden Kontaktflächendruckes bis zu dessen Verschwinden vollends zu. Der Gültigkeitsbereich einer Funktion ergibt sich dabei aus den Koordinaten der ersten Spalte bzw. Zeile in Breiten-/Fahrtrichtung bis hin zur letzten Spalte bzw. Zeile, die mindestens einen Pixel mit einem Druckwert ungleich Null aufweisen.

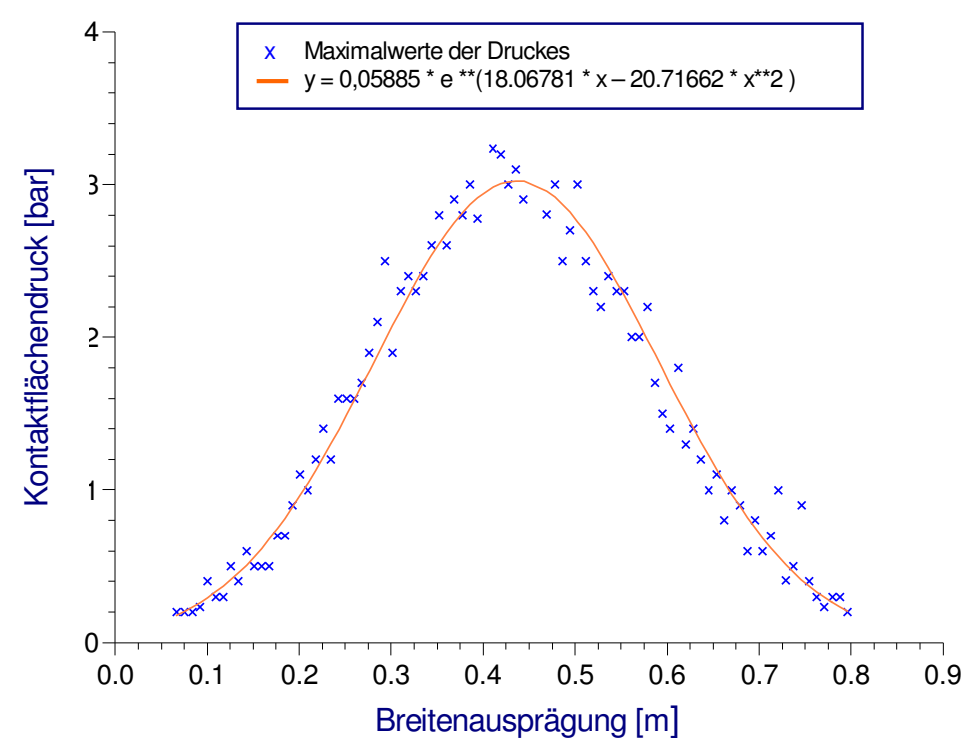

Abbildung 46: Ausgleich des maximalen Kontaktflächendruckes in Breitenrichtung (Reifen T22, Radlast 30 kN, Fülldruck 3,0 bar)

Ein Vergleich der Ausgleichsfunktionen aller Reifen zeigt für die beobachteten Maximalwerte eine gute bis sehr gute Anpassung. Die Bestimmtheitsmaße bewegen sich im Mittel bei $92 \%$ und belegen damit annehmbare Prognoseresultate. Die Standardabweichungen für Breitenund Fahrtrichtung sind mit Werten von 0,044 \% bzw. 0,062 \% sehr gering.

Mit dem dargelegten Modellansatz ist es also möglich, jede Kontaktflächendruckverteilung zu beschreiben und gültige Vorhersagen für das gesamte Reifenkollektiv zu entwickeln.

Der Verlauf der Funktion hängt dabei von der Größe der Konstanten a und c ab. Eine Variation des Wertes der Konstante a verändert y und damit das Maximum der Funktion. Das heißt, wenn c konstant ist, bewirkt eine Vergrößerung von a eine proportionale Vergrößerung der Kurve. Da die Ausgleichsfunktion einen konkaven Verlauf aufweist, sind die c-Werte durchweg negativ. Sofern die Konstante a nicht variiert, bewirkt ein größerer (absolut gesehen kleinerer) c-Wert ein Flacherwerden der Glockenkurve.

Die Lage innerhalb des Koordinatensystems wird durch die Konstante b bestimmt. Wie in Kapitel 7.3 beschrieben, stehen in Breitenrichtung $\mathrm{x}_{0}, \mathrm{x}_{1}, \mathrm{x}_{2} . . \mathrm{x}_{103}$ und in Fahrtrichtung $\mathrm{y}_{0}, \mathrm{y}_{1}, \mathrm{y}_{2} .$. 
$\mathrm{y}_{175}$ Pixel zur Verfügung. Das Maximum der Ausgleichsfunktion ist aber nicht immer identisch mit dem Zentrum der Messmatrix. Zur Lokalisierung wird deshalb mittels der Gleichung

$$
x=-\frac{b}{2 \cdot c}
$$

die Position des Funktionsmaximums ermittelt. Um diesen resultierenden Betrag wird das Koordinatensystem derart verschoben, dass das Funktionsmaximum über $\mathrm{x}$ gleich Null liegt. Der Regressor $x$ fällt weg, so dass für $b=0$ die Ordinate zur Symmetrieachse wird. Mit der Verlagerung des Koordinatenursprungs ins Funktionsmaximum ergeben sich demzufolge positive und negative x-Koordinaten (Abb. 47). Dieses Vorgehen führt zur Eliminierung der Konstanten b und zur Gleichsetzung des Wertes der Konstanten a mit dem Maximum der jeweiligen Druckverteilung. Da sich die Verteilung der auszugleichenden Punkteschar durch die Verschiebung auf der $\mathrm{x}$-Achse nicht verändert, bleiben auch die Bestimmtheitsmaße gleich.

$$
y=a \cdot e^{c \cdot x^{2}}
$$

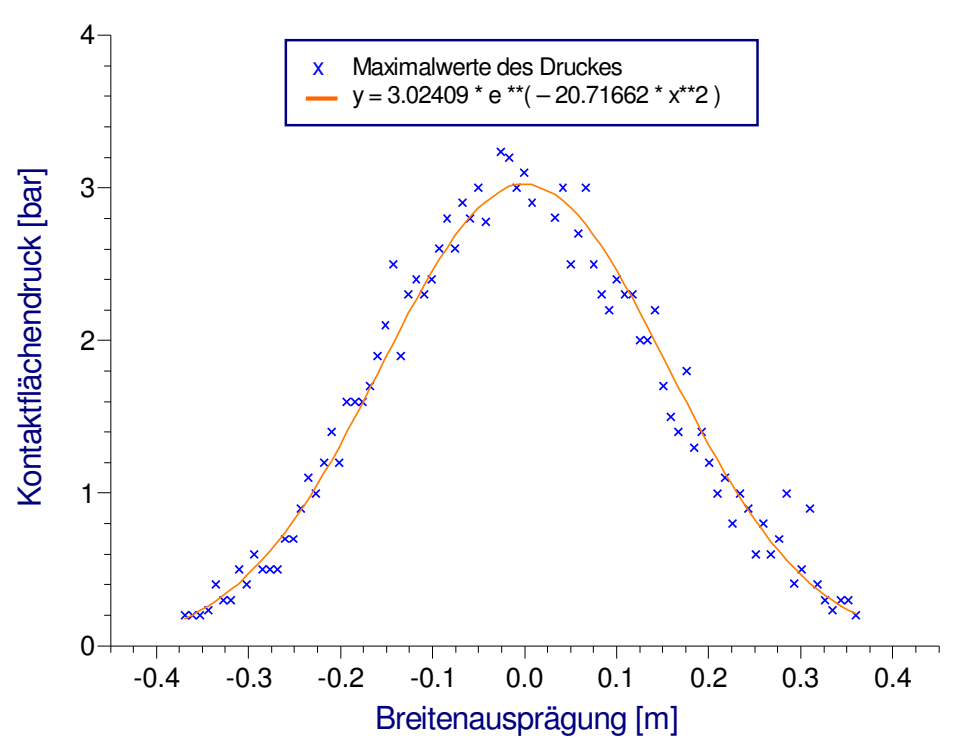

Abbildung 47: Ausgleich des maximalen Kontaktflächendruckes mit der Verschiebung des Koordinatenursprungs ins Funktionsmaximum (Reifen T22, Radlast 30 kN, Fülldruck 3,0 bar)

Die Verkürzung der Ausgleichsfunktion um die Konstante b ermöglicht ihre einfachere Handhabung und erlaubt es, die Druckverteilung eines jeden Reifens mit zwei Konstanten einer Modellgleichung zu beschreiben, die da lautet

$$
\text { Druck }=\mathbf{a}^{*} \mathrm{e}^{\left(\mathbf{c}{ }^{*} \text { Reifendistanz } z^{2}\right)}+\varepsilon
$$


Bei dieser Schreibweise der Glockenkurven-Funktion kennzeichnet a den Betrag des Maximums (in unserem Modell also den jeweiligen Spitzendruck), während c (stets negativ!) mit der Breite der Glocke sich verkleinert. $\varepsilon$ bezeichnet die Reststreuung (Gesamtstreuung minus erklärte Streuung) und fällt äußert gering aus. Die folgende Abbildung 48 illustriert diese Zusammenhänge.

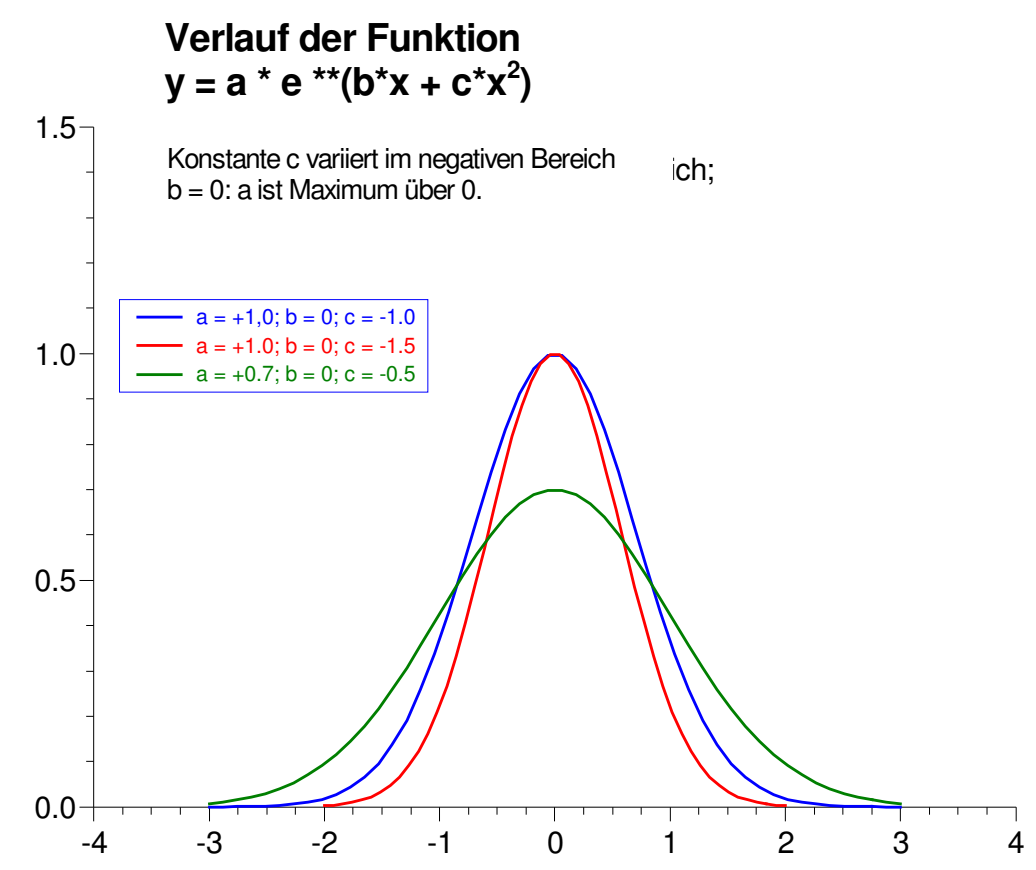

Abbildung 48: Darstellung der Zusammenhänge bei variierenden Werten der Konstanten a und $c$

\subsection{Regressionsanalytische Überprüfung der Druckverteilung}

Die vorhergehenden Ergebnisse zeigten, dass mit der Ausgleichsfunktion die unterschiedlichen Drücke, respektive die Druckverteilungen in der Kontaktfläche erfasst werden können. Die Verläufe der Druckverteilungsfunktionen in Breiten- (mit x indiziert) und Fahrtrichtung (mit y indiziert) werden durch die Konstanten a und $\mathrm{c}$ determiniert.

Regressionsanalysen sollten im Folgenden Aufschluss darüber geben, welche Variablen die einzelnen Konstanten beeinflussen:

1. Regressionen über $a_{x}$ und $a_{y}$ geben Auskunft über die Variablen, die den Spitzendruck beeinflussen.

2. Regressionen über $c_{x}$ und $c_{y}$ geben Auskunft über die Variablen, die einen steiler- oder flacherwerdenden Funktionsverlauf bzw. die Breiten- und Fahrtrichtungsausprägung beeinflussen.

Aus diesen Regressionsergebnissen lässt sich herleiten, welche Reifenparameter - neben den gezielt variierenden Größen Radlast und Fülldruck - die Druckverteilung determinieren und wie allgemein die Druckverteilung prognostiziert werden kann.

$\mathrm{Da}$ die innerhalb einer Last- und Fülldruckstufe betrachteten $a_{x}$ und $a_{y}$ sehr geringe ausgleichsbedingte Abweichungen zeigen, werden diese zu einem Mittelwert $a_{m}$ (Ausgleichsma- 
ximum) zusammengefasst. Die Konstanten $c_{x}$ und $c_{y}$ werden weiterhin getrennt betrachtet, so dass sich insgesamt drei Zielgrößen ergeben:

$$
\begin{aligned}
& a_{m}=\text { Konstante } a \text { in Breiten- und Fahrtrichtung } \\
& c_{x}=\text { Konstante } c \text { in Breitenrichtung } \\
& c_{y}=\text { Konstante } c \text { in Fahrtrichtung }
\end{aligned}
$$

Der Zusammenhang zwischen der abhängigen Variablen $\left(a_{m}, c_{x}, c_{y}\right)$ und den unabhängigen Variablen kann mit Hilfe von multiplen Regressionsmodellen quantifiziert werden. Dabei sei erwähnt, dass sich bei den Regressionen lediglich auf die Prüfung linearer Zusammenhänge beschränkt wurde.

Als unabhängige Variablen kommen zum einen die im Rahmen des Versuchsaufbaus vorab definierten Fülldrücke und Radlasten (s. Kap. 4.4) und zum anderen die konstruktiv vorgegeben und damit kennzeichnenden Parameter eines Reifens in Frage. Hierzu zählen die Nennbreite [mm], der Durchmesser [mm], die Tragfähigkeit ${ }^{26}[\mathrm{~kg}]$, die Reifenart (Niederquerschnittoder Standardreifen), die Bauweise (diagonal oder radial) und die Profilierung (Traktion- oder Softprofil). Die in Betracht kommenden Modellvariablen sind der Übersicht halber in Tabelle 15

\begin{tabular}{|c|c|c|c|}
\hline \multicolumn{2}{|c|}{ Zielgröße } & \multicolumn{2}{|c|}{ Regressoren } \\
\hline Verbal & Abkürzung & Verbal & Abkürzung \\
\hline $\begin{array}{l}\text { Ausgleichsmaximum } \\
\text { der Druckverteilungs- } \\
\text { funktion }\end{array}$ & $a_{m}$ & $\begin{array}{l}\text { Fülldruck } \\
\text { Radlast } \\
\text { Nennbreite } \\
\text { Durchmesser }\end{array}$ & $\begin{array}{l}\text { fuelldr } \\
\text { last } \\
\text { breite } \\
\text { durchm }\end{array}$ \\
\hline $\begin{array}{l}\text { Breiten- und Fahrtrich- } \\
\text { tungsausprägung der } \\
\text { Druckverteilungsfunk- } \\
\text { tion }\end{array}$ & $\begin{array}{l}c_{x} \\
c_{y}\end{array}$ & $\begin{array}{l}\text { Reifenart } \\
\text { Bauweise } \\
\text { Profilierung }\end{array}$ & $\begin{array}{l}\text { nqst } \\
\text { bauw } \\
\text { profil }\end{array}$ \\
\hline
\end{tabular}
dargestellt.

Tabelle 15: Variablen des Regressionsmodells

Um in die Regressionsanalyse aufgenommen zu werden, ist normalhin Intervallskalenniveau erforderlich. Dies folgt bereits aus dem Grundgedanken der Regression, nach dem Abstände kontinuierlich gemessen und interpretiert werden sollen (BROsIUS, 1998). Allerdings gibt es von dieser Regel eine Ausnahme. Bei bestimmten Fragestellungen ist es möglich, nominalskalierte Variablen als erklärende Variablen in das Modell aufzunehmen. In diesem Fall sind es die Variablen Reifenart, Bauweise und Profilierung, denen keine direkten Werte zugeordnet werden können. Um sie dennoch in die Regressionsanalyse einzubeziehen, besteht die Voraussetzung, dass die Variable zwei unterschiedliche Werte (0 und 1$)$ enthält. Eine solche Variable

${ }^{26}$ Wie in Kapitel 1.3.1.5 beschrieben, werden die Tragfähigkeiten derzeit durch die Angabe des Load-Index (LI) in Verbindung mit dem Speed-Index (SI) gekennzeichnet. Ein Teil der Reifen war nur mit der früheren Kennzeichnung, der PR-Zahl (Ply Rating, ein Festigkeitskode für die Karkasse, der allmählich durch die Kombination LI/Si ersetzt wird), versehen. Allerdings werden auch für diese Reifen nach Angabe der Hersteller Tragfähigkeiten in Abhängigkeit von Geschwindigkeiten ausgewiesen. Diese Tragfähigkeiten konnten entsprechenden Load-Indizes zugeordnet werden. Dabei orientierte sich die Zuordnung der Tragfähigkeiten an einem Speed-Index, der für alle Reifen zur Verfügung stand $(A 8=40 \mathrm{~km} / \mathrm{h})$. Mit diesem Vorgehen wurde eine einheitliche Basis für die zu untersuchenden Reifen geschaffen, um somit die Variable Tragfähigkeit in eine regressionsanalytische Untersuchung einfließen zu lassen. 
wird als Dummy-Variable bezeichnet. Bei der Dummy-Variablen Reifenart steht die 0 für die in dem Datensatz befindlichen Niederquerschnittreifen, die 1 für die Standardreifen. Unter der Bauweise werden diagonal (0) und radial (1) gebaute Reifen verstanden. Die Profilierung von Reifen wird in Traktions- (0) und Softprofilen (1) unterschieden. Hierbei interessiert vor allem, ob diese Variablen einen bestimmten Einfluss haben und ggf. signifikante Unterschiede bestehen.

\subsection{Multiple Schrittweise Regression}

Das Programm-Paket SPSS bietet nach BACKHAUS ET AL. (2000) u. a. eine Reihe von Möglichkeiten, um aus einer Menge von unabhängigen Variablen unterschiedliche Kombinationen auszuwählen und somit unterschiedliche Regressionsmodelle zu formulieren. Dabei orientiert sich das Programm im wesentlichen an dem Kriterium, ob eine Variable einen signifikanten Erklärungsbeitrag leistet oder nicht. Hierfür stehen mehrere automatische Auswahlverfahren (Vorwärts, Rückwärts und Schrittweise) zur Verfügung. Für die weitere regressionsanalytische Untersuchung wurde die Schrittweise Methode gewählt. Bei dem Berechnungsmodus der multiplen Schrittweisen Regression werden die unabhängigen Variablen einzeln nacheinander in die Regressionsgleichung einbezogen. Im ersten Schritt wird eine einfache Regression mit derjenigen Variablen durchgeführt, die die höchste Korrelation mit der abhängigen Variablen aufweist. Die sich ergebenden Residuen bilden die Zielgröße für den Rest der verbliebenden Regressorenschar. Die hieraus resultierende Anpassung wird durch den partiellen Korrelationskoeffizienten quantifiziert. Diese Methode wiederholt sich, bis alle der Routine angebotenen unabhängigen Variablen abgearbeitet wurden. Aus der Rangfolge der Aufnahme lässt sich die statistische Wichtigkeit der Variablen erkennen. Auf einem vordefinierten Signifikanzniveau entscheidet der F-Wert über Ein- oder Ausschluss der Variablen. Eine Variable wird nur dann aufgenommen, wenn der F-Wert ihres partiellen Korrelationskoeffizienten eine vorgegebene Schwelle $^{27}$ (FIN) übersteigt. Umgekehrt wird eine Variable bei Unterschreiten der Grenze für die F-Prüfgröße (FOUT) ${ }^{28}$ eliminiert (BACKHAUS ET AL., 2000).

Diese Vorgehensweise wiederholt sich solange, bis alle Variablen auf Signifikanz geprüft wurden. Sinkt durch die schrittweise Einbeziehung von weiteren unabhängigen Variablen der F-Wert einer sich im Modell befindlichen Variablen unter die Signifikanzschwelle, wird diese Variable wieder aus dem Modell eliminiert und erscheint erneut in der Liste der bisher noch nicht berücksichtigten Variablen. Von dort kann sie, falls ihr F-Wert wieder signifikant wird, erneut in das Regressionsmodell übernommen werden.

Auf einem 95 \%igen Signifikanzniveau (Irrtumswahrscheinlichkeit von 5 \%) wurden die Regressoren mittels Anwendung der Schrittweisen Methode getestet und entsprechend in der Modellbildung berücksichtigt.

27 FIN = Schwellenwert für den F-Wert des partiellen Korrelationskoeffizienten bei Aufnahme einer Variablen. Voreingestellt ist der Wert $=3,84$.

${ }^{28}$ FOUT $=$ Schwellenwert für den F-Wert des partiellen Korrelationskoeffizienten bei der Elimination einer Variablen. Voreingestellt ist der Wert $=2,71$. 


\subsection{Wahl des Modells}

Generell sollte ein Regressionsmodell die Prognose der Zielgröße so präzise, aber auch so einfach wie möglich gestalten (JACKE, 1980). Die jeweilige Präzision drückt sich u. a. in der Höhe des jeweiligen Bestimmtheitsmaßes aus, dessen Änderungen bei schrittweisen Regressionen von den partiellen Korrelationen dirigiert werden. Zur Beurteilung fand das korrigierte Bestimmtheitsmaß Berücksichtigung, bei dessen Berechnung zusätzlich die Freiheitsgrade (Anzahl der bei der Schätzung verwendeten erklärenden Variablen) berücksichtigt werden. Neben dem korrigierten Bestimmtheitsmaß wurde sich zudem an der für jeden Regressionskoeffizienten ausgewiesenen Signifikanz orientiert.

Dabei wird es von der Präzision der in einer autoselektiven Regression durchgeführten Schätzung abhängen, welches der Modelle die genannten Voraussetzungen am besten erfüllt. Statistisch signifikante Zusammenhänge sollten vom Untersucher nur dann akzeptiert werden, wenn sie seinen sachlogischen Erwartungen entsprechen und keine Widersprüche aufweisen (BACKHAUS ET AL., 2000).

\subsection{Regression für die Konstante $a_{m}$}

In einem ersten Schritt wurde die Konstante $a_{m}$ untersucht. Die Regressionsergebnisse sind in Tabelle 16 zu finden.

Tabelle 16: Ergebnisse der multiplen Regressionsanalyse mit der abhängigen Variablen $a_{m}$

Modellzusammenfassung ${ }^{9}$

\begin{tabular}{l|r|r|r|r|}
\hline Modell & R & R-Quadrat & $\begin{array}{r}\text { Korrigiertes } \\
\text { R-Quadrat }\end{array}$ & $\begin{array}{r}\text { Standard- } \\
\text { fehler des } \\
\text { Schätzers }\end{array}$ \\
\hline 1 &, $732(\mathrm{a})$ &, 536 &, 536 &, 95843 \\
2 &, $873(\mathrm{~b})$ &, 763 &, 763 &, 68571 \\
3 &, $882(\mathrm{c})$ &, 778 &, 778 &, 66311 \\
4 &, $885(\mathrm{~d})$ &, 782 &, 782 &, 65693 \\
5 &, $885(\mathrm{e})$ &, 783 &, 783 &, 65566 \\
6 &, $885(\mathrm{f})$ &, 784 &, 784 &, 65467 \\
\hline
\end{tabular}
a Einflussvariablen: (Konstante), Radlast [kN]
b Einflussvariablen: (Konstante), Radlast [kN], Fülldruck [bar]
c Einflussvariablen: (Konstante), Radlast [kN], Fülldruck [bar], Nennbreite [mm]
d Einflussvariablen: (Konstante), Radlast [kN], Fülldruck [bar], Nennbreite [mm], Durchmesser [mm]
e Einflussvariablen: (Konstante), Radlast [kN], Fülldruck [bar], Nennbreite [mm], Durchmesser [mm],
Tragfähigkeit [kg]
f Einflussvariablen: (Konstante), Radlast [kN], Fülldruck [bar], Nennbreite [mm], Durchmesser [mm],
Tragfähigkeit [kg], Profilart
g Abhängige Variable : Konstante am des Maximalwertausgleiches


Koeffizienten $^{\mathrm{a}}$

\begin{tabular}{|c|c|c|c|c|c|c|}
\hline & \multirow{2}{*}{ Modell } & \multicolumn{2}{|c|}{$\begin{array}{l}\text { Nicht standardisierte } \\
\text { Koeffizienten }\end{array}$} & \multirow{2}{*}{$\begin{array}{c}\begin{array}{c}\text { Standardisierte } \\
\text { Koeffizienten }\end{array} \\
\text { Beta }\end{array}$} & \multirow[t]{2}{*}{$\mathrm{T}$} & \multirow[t]{2}{*}{ Signifikanz } \\
\hline & & B & $\begin{array}{l}\text { Standard- } \\
\text { fehler }\end{array}$ & & & \\
\hline \multirow[t]{2}{*}{1} & (Konstante) & ,913 & ,045 & & 20,074 & ,000 \\
\hline & Radlast [kN] & ,073 & ,001 & ,732 & 52,982 & ,000 \\
\hline \multirow[t]{3}{*}{$\overline{2}$} & (Konstante) &,- 153 & ,039 & & $-3,881$ & ,000 \\
\hline & Radlast [kN] & ,073 & ,001 & ,729 & 73,686 & ,000 \\
\hline & Fülldruck [bar] & ,441 & ,009 & ,476 & 48,119 &, 000 \\
\hline \multirow[t]{4}{*}{3} & (Konstante) & ,916 & ,091 & & 10,116 & ,000 \\
\hline & Radlast [kN] & ,073 & ,001 & ,729 & 76,265 &, 000 \\
\hline & Fülldruck [bar] & ,441 & 009 & ,475 & 49,679 &, 000 \\
\hline & Nennbreite [mm] &,- 002 & ,000 &,- 124 & $-13,007$ &, 000 \\
\hline \multirow[t]{5}{*}{4} & (Konstante) & 1,564 & ,130 & & 11,993 & ,000 \\
\hline & Radlast [kN] &, 073 & ,001 & ,729 & 76,948 &, 000 \\
\hline & Fülldruck [bar] & ,441 & 009 & ,476 & 50,221 &, 000 \\
\hline & Nennbreite [mm] &,- 002 & ,000 &,- 120 & $-12,658$ &, 000 \\
\hline & Durchmesser [mm] & ,000 & ,000 &,- 065 & $-6,844$ &, 000 \\
\hline \multirow[t]{6}{*}{5} & (Konstante) & 1,759 & ,143 & & 12,256 &, 000 \\
\hline & Radlast [kN] & ,073 & ,001 & ,729 & 77,100 &, 000 \\
\hline & Fülldruck [bar] & ,441 &, 009 & ,476 & 50,316 & ,000 \\
\hline & Nennbreite [mm] &,- 002 &, 000 &,- 147 & $-11,628$ &, 000 \\
\hline & Durchmesser [mm] &,- 001 & ,000 &,- 082 & $-7,564$ &, 000 \\
\hline & Tragfähigkeit [kg] & 5,627E-05 & ,000 & ,045 & 3,224 & ,001 \\
\hline \multirow[t]{7}{*}{6} & (Konstante) & 1,820 & ,145 & & 12,567 & 0,000 \\
\hline & Radlast [kN] & ,073 &, 001 & ,729 & 77,219 &, 000 \\
\hline & Fülldruck [bar] & ,441 & ,009 & ,476 & 50,391 &, 000 \\
\hline & Nennbreite [mm] &,- 002 &, 000 &,- 142 & $-11,086$ &, 000 \\
\hline & Durchmesser [mm] &,- 001 &, 000 &,- 093 & $-8,107$ &, 000 \\
\hline & Tragfähigkeit [kg] & $6,588 \mathrm{E}-05$ & ,000 & ,052 & 3,713 &, 000 \\
\hline & &,- 095 & ,033 &,- 031 & $-2,891$ & ,004 \\
\hline
\end{tabular}

a Abhängige Variable: Konstante am des Maximalwertausgleiches

Die Betrachtung des in der Modellzusammenfassung ausgewiesenen korrigierten Bestimmtheitsmaßes in Tabelle 16 zeigt, dass allein die Radlast mit 53,6 \% die Varianz der Zielgröße erklärt. Im zweiten Modell liefert die einbezogene Variable Fülldruck einen Erklärungsbeitrag von $22,7 \%$. Die im dritten und vierten Modell aufgenommenen Variablen Nennbreite und Durchmesser erhöhen das Bestimmtheitsmaß von 76,3 \% auf 78,2 \%. Hingegen erhöht sich das Bestimmtheitsmaß im fünften und sechsten Modell kaum noch. Sein Zuwachs für die aufgenommen Variablen Tragfähigkeit und Profilart beziffert sich für jedes Modell auf lediglich 0,1\%. Da sich die Variablen Tragfähigkeit und Profilart mit ihren Interpretationspotentialen im Promillebereich bewegen, werden diese - zu Gunsten einer überschaubaren und nach Wichtigkeit gestaffelten Deutung des Modells - aus weiteren Betrachtungen ausgeschlossen. Die vier berücksichtigten Variablen erklären mit $78 \%$ die Zielgröße und damit die Beeinflussung des Spitzendruckes. Die Überprüfung der Regressionskoeffizienten insgesamt zeigt, dass sich alle Koeffizienten deutlich von Null unterscheiden. 
Das vierte Regressionsmodell führt die bedeutsamen Regressoren zur Erklärung der abhängigen Variablen $a_{m}$ auf. Die Gleichung kann in allgemeiner Form folgendermaßen ausgedrückt werden (Tab. 17):

Tabelle 17: Regressionsgleichung für die Zielgröße $a_{m}$

\begin{tabular}{|c|c|c|c|c|c|c|c|}
\hline \multirow{2}{*}{ Zielgröße } & \multirow{2}{*}{ Konstante } & \multirow{2}{*}{\multicolumn{4}{|c|}{ Regressionskoeffizienten }} & \multicolumn{2}{|c|}{ Kenngrößen } \\
\hline & & & & & & B & Signifikanz \\
\hline$a_{m}$ & $+1,56360$ & $\begin{array}{c}\text { Radlast } \\
+0,07263\end{array}$ & $\begin{array}{r}\text { Fülldruck } \\
+0,44139\end{array}$ & $\begin{array}{c}\text { Nennbreite } \\
-0,00174\end{array}$ & $\begin{array}{c}\text { Durchmesser } \\
-0,00048\end{array}$ & 0,78 & 0,000 \\
\hline
\end{tabular}

Das Bestimmtheitsmaß erlaubt lediglich Aussagen über die Treffsicherheit des Modells. Für den Verlauf der Regressionsfunktion sind jedoch die Regressionskoeffizienten der einzelnen Regressoren entscheidend. Die Regressoren Radlast und Fülldruck weisen positive, Nennbreite und Durchmesser jeweils negative Vorzeichen auf. Die Vorzeichen liefern folgende Erklärung: mit steigender Radlast und Zunahme des Fülldruckes nimmt der Wert von $\mathrm{a}_{\mathrm{m}}$ und damit der Spitzendruck zu. Werden Nennbreite und Durchmesser mit einbezogen, so kommt man abschließend zu folgendem Ergebnis: Je größer die Nennbreite und der Durchmesser eines Reifens, desto kleiner wird bei Anwendung der Regressionsgleichung die Zielgröße $\mathrm{a}_{\mathrm{m}}$. Diese Überlegungen zeigen, dass die Vorzeichen auf ein insgesamt plausibles Regressionsmodell hinweisen.

\subsection{Regression für die Konstante $c_{x}$}

In einem zweiten Schritt wurde die Konstante $c_{x}$, die die Breitenausprägung determiniert, untersucht. Die Regressionsergebnisse sind in der Tabelle 18 dargestellt.

Tabelle 18: Ergebnisse der multiplen Regressionsanalyse mit der abhängigen Variablen $c_{x}$

Modellzusammenfassung ${ }^{\mathrm{g}}$

\begin{tabular}{|l|r|r|r|r|}
\hline Modell & $\mathrm{R}$ & R-Quadrat & $\begin{array}{r}\text { Korrigiertes } \\
\text { R-Quadrat }\end{array}$ & $\begin{array}{l}\text { Standard- } \\
\text { fehler des } \\
\text { Schätzers }\end{array}$ \\
\hline 1 &, $617(\mathrm{a})$ &, 381 &, 381 & 7,8723690 \\
2 &, $775(\mathrm{~b})$ &, 600 &, 600 & 6,3317842 \\
3 &, $836(\mathrm{c})$ &, 699 &, 698 & 5,4961392 \\
4 &, $837(\mathrm{~d})$ &, 700 &, 700 & 5,4829627 \\
5 &, $839(\mathrm{e})$ &, 704 &, 703 & 5,4502626 \\
6 &, $840(\mathrm{f})$ &, 706 &, 706 & 5,4293441 \\
\hline
\end{tabular}

a Einflussvariablen: (Konstante), Nennbreite [mm]

b Einflussvariablen : (Konstante), Nennbreite [mm], Fülldruck [bar]

c Einflussvariablen : (Konstante), Nennbreite [mm], Fülldruck [bar], Radlast [kN]

d Einflussvariablen : (Konstante), Nennbreite [mm], Fülldruck [bar], Radlast [kN], Durchmesser [mm]

e Einflussvariablen : (Konstante), Nennbreite [mm], Fülldruck [bar], Radlast [kN], Durchmesser [mm],

Tragfähigkeit [kg]

f Einflussvariablen : (Konstante), Nennbreite [mm], Fülldruck [bar], Radlast [kN], Durchmesser [mm],

Tragfähigkeit [kg], Reifenart

g Abhängige Variable : Konstante cx des Maximalwertausgleiches 
Koeffizienten $^{\mathrm{a}}$

\begin{tabular}{|c|c|c|c|c|c|c|}
\hline & \multirow[t]{2}{*}{ Modell } & \multicolumn{2}{|c|}{$\begin{array}{l}\text { Nicht standardisierte } \\
\text { Koeffizienten }\end{array}$} & \multirow{2}{*}{$\begin{array}{c}\begin{array}{c}\text { Standardisierte } \\
\text { Koeffizienten }\end{array} \\
\text { Beta }\end{array}$} & \multirow[t]{2}{*}{$\mathrm{T}$} & \multirow[t]{2}{*}{ Signifikanz } \\
\hline & & B & $\begin{array}{l}\text { Standard- } \\
\text { fehler }\end{array}$ & & & \\
\hline \multirow[t]{2}{*}{1} & (Konstante) & $-63,953$ & ,989 & & $-64,664$ &, 000 \\
\hline & Nennbreite [mm] &, 064 & ,002 & ,617 & 38,671 &, 000 \\
\hline \multirow[t]{3}{*}{2} & (Konstante) & $-56,257$ & ,823 & & $-68,350$ &, 000 \\
\hline & Nennbreite [mm] &, 064 & ,001 & ,615 & 47,860 &, 000 \\
\hline & Fülldruck [bar] & $-3,084$ & ,085 &,- 468 & $-36,410$ &, 000 \\
\hline \multirow[t]{4}{*}{3} & (Konstante) & $-62,767$ & ,751 & & $-83,596$ &, 000 \\
\hline & Nennbreite [mm] &, 063 & ,001 & ,613 & 54,987 &, 000 \\
\hline & Fülldruck [bar] & $-3,100$ & ,074 &,- 470 & $-42,160$ & ,000 \\
\hline & Radlast [kN] & ,223 & ,008 & ,314 & 28,192 &, 000 \\
\hline \multirow[t]{5}{*}{4} & (Konstante) & $-65,576$ & 1,088 & & $-60,265$ &, 000 \\
\hline & Nennbreite [mm] & ,063 &, 001 & ,610 & 54,772 & ,000 \\
\hline & Fülldruck [bar] & $-3,103$ & ,073 &,- 470 & $-42,299$ &, 000 \\
\hline & Radlast [kN] & ,223 & ,008 & ,314 & 28,277 &, 000 \\
\hline & Durchmesser [mm] & ,002 & ,001 & ,040 & 3,559 &, 000 \\
\hline \multirow[t]{6}{*}{5} & (Konstante) & $-68,337$ & 1,193 & & $-57,297$ &, 000 \\
\hline & Nennbreite [mm] &, 069 & ,002 & ,664 & 44,874 & ,000 \\
\hline & Fülldruck [bar] & $-3,103$ & ,073 &,- 470 & $-42,549$ & ,000 \\
\hline & Radlast [kN] & ,223 & ,008 & ,314 & 28,441 &, 000 \\
\hline & Durchmesser [mm] &, 004 &, 001 &, 073 & 5,771 &, 000 \\
\hline & Tragfähigkeit [kg] &,- 001 & ,000 &,- 089 & $-5,493$ &, 000 \\
\hline \multirow[t]{7}{*}{6} & (Konstante) & $-67,749$ & 1,195 & & $-56,672$ & 000 \\
\hline & Nennbreite [mm] &, 065 & ,002 & ,632 & 38,524 &, 000 \\
\hline & Fülldruck [bar] & $-3,104$ & ,073 &,- 471 & $-42,729$ &, 000 \\
\hline & Radlast $[\mathrm{kN}]$ & ,223 &, 008 & ,315 & 28,561 &, 000 \\
\hline & Durchmesser [mm] &, 005 & ,001 & ,096 & 7,057 & ,000 \\
\hline & Tragfähigkeit [kg] &,- 001 &, 000 &,- 091 & $-5,632$ &, 000 \\
\hline & & $-1,565$ & ,353 &,- 062 & $-4,439$ & ,000 \\
\hline
\end{tabular}

a Abhängige Variable: Konstante cx des Maximalwertausgleiches

Für die untersuchte Zielgröße $c_{x}$ liefert die Nennbreite mit 38,1 \% den größten Beitrag zur Varianzerklärung. Mit der im zweiten Schritt aufgenommenen Variable Fülldruck erhöht sich das korrigierte Bestimmtheitsmaß um 21,9\% auf 60,0 \% (Tab. 18). Die Aufnahme der Variable Radlast im dritten Modell liefert mit 9,9\% einen weiteren ausreichenden Erklärungsbeitrag der Zielgröße. Eine marginale Erhöhung des korrigierten Bestimmtheitsmaßes leisten die in den nachfolgenden Modellen vier, fünf und sechs aufgeführten Variablen Durchmesser, Tragfähigkeit und Reifenart. Aufgrund des insgesamt geringen Einflusses dieser Variablen auf die Zielgröße, wurde aus Praktikabilitätsgründen auf eine weitere Verwendung verzichtet. Die Überprüfung der Regressionskoeffizienten zeigt, dass sich alle Koeffizienten deutlich von Null unterscheiden.

Mit einem Bestimmtheitsmaß von $70 \%$ weist die in Tabelle 19 dargestellte Regressionsgleichung annehmbare Prognoseresultate auf: 
Tabelle 19: Regressionsgleichung für die Zielgröße $c_{x}$

\begin{tabular}{|c|c|c|c|c|c|c|}
\hline \multirow{2}{*}{ Zielgröße } & \multirow{2}{*}{ Konstante } & \multicolumn{3}{|c|}{ Regressionskoeffizienten } & \multicolumn{2}{c|}{ Kenngrößen } \\
\cline { 5 - 7 } & & \multicolumn{2}{|c|}{ B } & Signifikanz \\
\hline$c_{X}$ & $-62,76699$ & $\begin{array}{c}\text { Nennbreite } \\
+0,06346\end{array}$ & $\begin{array}{c}\text { Fülldruck } \\
-3,09995\end{array}$ & $\begin{array}{c}\text { Radlast } \\
+0,22264\end{array}$ & 0,70 & 0,000 \\
\hline
\end{tabular}

Werden an dieser Stelle die Regressionskoeffizienten der einzelnen Gleichungen betrachtet, so zeigt sich, dass die Konstante $c_{x}$ in Breitenrichtung ausschließlich von den Regressoren Nennbreite, Fülldruck und Radlast determiniert wird. Wie in Kapitel 7.3 festgestellt, nehmen die Werte für $c\left(c_{x}\right)$ negative Vorzeichen an. Eine Interpretation der Vorzeichen der Regressionskoeffizienten lässt erkennen, dass mit Zunahme der Nennbreite die Konstante $c_{x}$ größer, der Kurvenverlauf insgesamt flacher wird. Dasselbe gilt für die Radlast. Das negative Vorzeichen des Fülldruckes bewirkt hingegen eine Verkleinerung der Konstanten $\mathrm{c}_{\mathrm{x}}$.

\subsection{Regression für die Konstante $c_{y}$}

Der dritte Schritt beinhaltete die Untersuchung der Konstanten $c_{y}$, die den Funktionsverlauf in Fahrtrichtung beeinflusst. Die Regressionsergebnisse sind in der Tabelle 20 aufgeführt.

Tabelle 20: Ergebnisse der multiplen Regressionsanalyse mit der abhängigen Variablen $c_{y}$

Modellzusammenfassung ${ }^{g}$

\begin{tabular}{|l|r|r|r|l|}
\hline Modell & R & R-Quadrat & $\begin{array}{c}\text { Korrigiertes } \\
\text { R-Quadrat }\end{array}$ & $\begin{array}{c}\text { Standard- } \\
\text { fehler des } \\
\text { Schätzers }\end{array}$ \\
\hline 1 &, $571(\mathrm{a})$ &, 326 &, 325 & 8,8605641 \\
2 &, $776(\mathrm{~b})$ &, 602 &, 602 & 6,8088451 \\
3 &, $818(\mathrm{c})$ &, 669 &, 669 & 6,2064377 \\
4 &, $839(\mathrm{~d})$ &, 704 &, 703 & 5,8758001 \\
5 &, $841(\mathrm{e})$ &, 708 &, 707 & 5,8378573 \\
6 &, $842(\mathrm{f})$ &, 708 &, 708 & 5,8329239 \\
\hline
\end{tabular}

a Einflussvariablen: (Konstante), Radlast [kN]

b Einflussvariablen: (Konstante), Radlast [kN], Fülldruck [bar]

c Einflussvariablen: (Konstante), Radlast [kN], Fülldruck [bar], Durchmesser [mm]

d Einflussvariablen: (Konstante), Radlast [kN], Fülldruck [bar], Durchmesser [mm], Nennbreite [mm]

e Einflussvariablen: (Konstante), Radlast [kN], Fülldruck [bar], Durchmesser [mm], Nennbreite [mm], Bauweise

f Einflussvariablen: (Konstante), Radlast [kN], Fülldruck [bar], Durchmesser [mm], Nennbreite [mm], Bauweise,

Profilart

g Abhängige Variable: Konstante cy des Maximalwertausgleiches 
Koeffizienten $^{\mathrm{a}}$

\begin{tabular}{|c|c|c|c|c|c|c|}
\hline & \multirow[t]{2}{*}{ Modell } & \multicolumn{2}{|c|}{$\begin{array}{l}\text { Nicht standardisierte } \\
\text { Koeffizienten }\end{array}$} & \multirow{2}{*}{$\begin{array}{c}\begin{array}{c}\text { Standardisierte } \\
\text { Koeffizienten }\end{array} \\
\text { Beta }\end{array}$} & \multirow[t]{2}{*}{$\mathrm{T}$} & \multirow[t]{2}{*}{ Signifikanz } \\
\hline & & B & $\begin{array}{l}\text { Standard- } \\
\text { fehler }\end{array}$ & & & \\
\hline \multirow[t]{2}{*}{1} & (Konstante) & $-36,291$ & ,421 & & $-86,279$ & ,000 \\
\hline & Radlast [kN] & ,436 & ,013 &, 571 & 34,224 &, 000 \\
\hline \multirow[t]{3}{*}{2} & (Konstante) & $-27,263$ & ,391 & & $-69,727$ & ,000 \\
\hline & Radlast [kN] &, 439 & ,010 &, 575 & 44,848 &, 000 \\
\hline & Fülldruck [bar] & $-3,738$ & 091 &,- 526 & $-41,037$ &, 000 \\
\hline \multirow[t]{4}{*}{3} & (Konstante) & $-48,205$ & 1,007 & & $-47,885$ & ,000 \\
\hline & Radlast [kN] & ,440 & ,009 &, 576 & 49,303 &, 000 \\
\hline & Fülldruck [bar] & $-3,758$ & 083 &,- 529 & $-45,259$ &, 000 \\
\hline & Durchmesser [mm] &, 015 & ,001 & ,260 & 22,244 &, 000 \\
\hline \multirow[t]{5}{*}{4} & (Konstante) & $-36,930$ & 1,166 & & $-31,670$ & ,000 \\
\hline & Radlast [kN] & ,440 & ,008 &, 577 & 52,170 &, 000 \\
\hline & Fülldruck [bar] & $-3,767$ & 079 &,- 530 & $-47,919$ &, 000 \\
\hline & Durchmesser [mm] &, 016 & 001 & ,272 & 24,536 &, 000 \\
\hline & Nennbreite [mm] &,- 021 & ,001 &,- 186 & $-16,781$ &, 000 \\
\hline \multirow[t]{6}{*}{5} & (Konstante) & $-36,526$ & 1,161 & & $-31,469$ & ,000 \\
\hline & Radlast [kN] & ,440 & ,008 &, 576 & 52,467 &, 000 \\
\hline & Fülldruck [bar] & $-3,762$ & ,078 &,- 529 & $-48,166$ & ,000 \\
\hline & Durchmesser [mm] & ,017 & ,001 & ,287 & 25,344 &, 000 \\
\hline & Nennbreite [mm] &,- 023 & ,001 &,- 206 & $-17,826$ &, 000 \\
\hline & Bauweise & $-1,881$ & ,329 &,- 067 & $-5,711$ & ,000 \\
\hline \multirow[t]{7}{*}{6} & (Konstante) & $-36,751$ & 1,164 & & $-31,573$ & 0,000 \\
\hline & Radlast [kN] & ,440 & ,008 &, 576 & 52,513 &, 000 \\
\hline & Fülldruck [bar] & $-3,763$ & ,078 &,- 529 & $-48,211$ &, 000 \\
\hline & Durchmesser [mm] &, 017 & ,001 & ,294 & 25,142 &, 000 \\
\hline & Nennbreite [mm] &,- 024 & ,001 &,- 214 & $-17,742$ &, 000 \\
\hline & Bauweise & $-1,749$ & ,334 &,- 063 & $-5,230$ &, 000 \\
\hline & & ,660 & ,292 & ,028 & 2,258 & ,024 \\
\hline
\end{tabular}

a Abhängige Variable: Konstante cy des Maximalwertausgleiches

Das korrigierte Bestimmtheitsmaß in der Modellzusammenfassung der Tabelle 20 zeigt, dass die Radlast mit 32,5 \% die Varianz der Zielgröße cy erklärt. Die Variable Fülldruck erhöht das Bestimmtheitsmaß um 27,7 \% auf 60,2 \%. Einen weiteren ausreichenden Beitrag zur Erklärung der Gesamtstreuung der Zielgröße cy mit 6,7 \% liefert die im dritten Modell aufgenommene Variable Durchmesser. Mit einem Erklärungsbeitrag von 3,4 \% ist die im vierten Modell einbezogene Variable Nennbreite ebenfalls in das Regressionsmodell aufzunehmen. Da die im fünften und sechsten Modell aufgenommenen Variablen Bauweise und Profilart im Vergleich zu den übrigen Variablen einen insgesamt geringen Einfluss auf die Zielgröße haben, wird auf deren Verwendung verzichtet. Auch hier sind die Regressionskoeffizienten von Null verschieden.

Mit einem Bestimmtheitsmaß von $70 \%$ werden gute Prognoseresultate erzielt. Die Regressionsgleichung wird in Tabelle 21 wie folgt beschrieben: 
Tabelle 21: Regressionsgleichung für die Zielgröße cy

\begin{tabular}{|c|c|c|c|c|c|c|c|}
\hline \multirow{2}{*}{ Zielgröße } & \multirow{2}{*}{ Konstante } & \multirow{2}{*}{\multicolumn{4}{|c|}{ Regressionskoeffizienten }} & \multicolumn{2}{|c|}{ Kenngrößen } \\
\hline & & & & & & B & Signifikanz \\
\hline cy & - 36,93026 & $\begin{array}{c}\text { Radlast } \\
+0,44048\end{array}$ & $\begin{array}{l}\text { Fülldruck } \\
-3,76698\end{array}$ & $\begin{array}{l}\text { Durchmesser } \\
+0,01564\end{array}$ & $\begin{array}{c}\text { Nennbreite } \\
-0,02075\end{array}$ & 0,70 & 0,000 \\
\hline
\end{tabular}

Determinieren die Nennbreite, der Fülldruck und die Radlast den Funktionsverlauf in Breitenrichtung, deuten sachlogische Überlegungen darauf hin, dass der Funktionsverlauf in Fahrtrichtung neben Radlast und Fülldruck durch den Durchmesser beeinflusst wird. Darüber hinaus wirkt sich aber auch die Nennbreite aus. Der Einfluss wird deutlich, wenn die Vorzeichen der Regressionskoeffizienten der einzelnen Variablen näher betrachtet werden: Demnach wird die Druckprofilsausprägung in Fahrtrichtung bei höheren Radlasten und bei größeren Raddurchmessern flacher, bei steigenden Fülldrücken und bei wachsenden Nennbreiten steiler. Der Einfluss der Nennbreite erklärt sich hierbei folgendermaßen. Wenn man ceteris paribus (gleiche Radlast, gleicher Fülldruck, gleicher Raddurchmesser) die Breite des Reifens vergröBert, so bekommt das Rad die Möglichkeit, einen gegebenen Druck auf mehr Fläche in der Breitenrichtung zu verteilen: die Druckintensität in Fahrtrichtung wird dabei abgebaut, $c_{y}$ sinkt. Das heißt, je schmaler ein Rad bzw. ein Reifen ist, desto eher wird sich bei einem gegebenen Druck dieser auf „mehr Fläche“ in Fahrtrichtung verteilen.

Nach Interpretation und Auswahl der Regressionsmodelle für die untersuchten Konstanten $\mathrm{a}_{\mathrm{m}}$, $c_{x}$ und $c_{y}$ wird deutlich, dass die Druckverteilung neben Radlast und Fülldruck durch die Reifenparameter Nennbreite und Durchmesser beeinflusst wird.

\subsection{Prognosemodell}

Das in den Untersuchungen berücksichtigte Kollektiv an Reifen deckt nahezu das gesamte Spektrum der derzeit in der forstlichen Praxis eingesetzten Dimensionen ab. Für alle Reifen deren Ausprägungen bei der Nennbreite und beim Durchmesser innerhalb der Grenzen des Testkollektivs liegen, ist es möglich, die in der Kontaktfläche herrschenden Drücke unter Kenntnis der Radlast und des Fülldruckes zu prognostizieren und so die erwartete Druckverteilung für einen Reifen darzustellen.

Grundlage hierfür sind die über die Regressionsgleichungen ermittelten Konstanten $\mathrm{a}_{\mathrm{m}}, \mathrm{c}_{\mathrm{x}}$ und $c_{y}$. Werden die Werte dieser Konstanten in die Formel der Ausgleichsfunktion - jeweils für Breiten- und Fahrtrichtung - eingesetzt, so können die Funktionsverläufe vorhergesagt werden. Die Ausprägungen der einzelnen Funktionsverläufe sollen anhand der nachfolgend dargestellten Abbildungen exemplarisch näher erläutert werden. Dabei werden Radlast und Fülldruck bei $30 \mathrm{kN}$ und 3,0 bar konstant gehalten. Die Reifenparameter Nennbreite bzw. Durchmesser variieren, um deren Einfluss auf die prognostizierten Druckverteilungen zu verdeutlichen. Hierzu wurden jeweils die unteren und oberen Grenzwerte der Reifenparameter des untersuchten Reifenkollektives gewählt. Diese betragen bei der Nennbreite $378 \mathrm{~mm}$ und $800 \mathrm{~mm}$ und für den Durchmesser $1020 \mathrm{~mm}$ und $1810 \mathrm{~mm}$.

Das Maximum ( $\mathrm{a}_{\mathrm{m}}$ ) einer Druckverteilungsfunktion wird (s. Kap. 7.7) mit Zunahme der Nennbreite und des Durchmessers reduziert, wie in den Abbildungen 50 und 52 zu sehen ist. 
Für die Breitenausprägung findet neben Radlast und Fülldruck nur die Nennbreite weitere Berücksichtigung. In Abbildung 49 zeigt die Funktion des Reifens mit 378 mm Nennbreite einen steileren Verlauf als die Funktion des Reifen mit 800 mm Nennbreite in Abbildung 50.

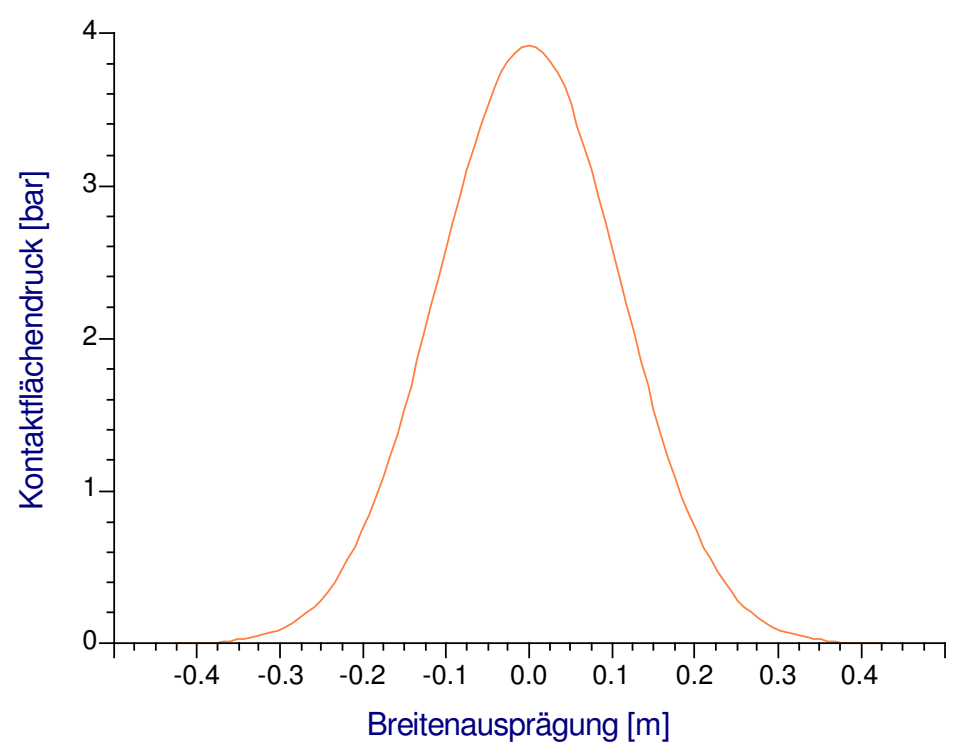

Abbildung 49: Prognose einer Druckverteilungsfunktion in Breitenrichtung (Radlast 30 kN, Fülldruck 3,0 bar, Nennbreite 378 mm, Durchmesser 1020 mm)

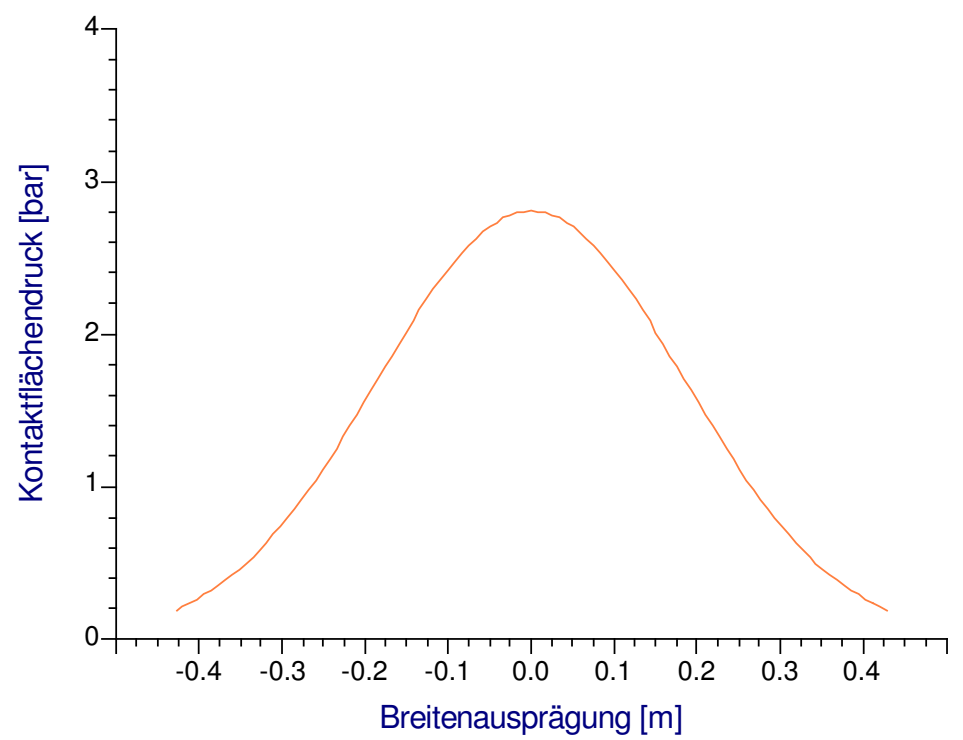

Abbildung 50: Prognose einer Druckverteilungsfunktion in Breitenrichtung (Radlast 30 kN, Fülldruck 3,0 bar, Nennbreite 800 mm, Durchmesser $1810 \mathrm{~mm}$ )

Die Funktionsmaxima in Fahrtrichtungsausprägung sind für die jeweiligen Reifen identisch mit denen in Breitenausprägung (vgl. Abb. 49 vs. 51 und Abb. 50 vs. 52). Sie unterscheiden sich offensichtlich in der Höhe. 
Die Fahrtrichtungsausprägung wird einerseits durch den Durchmesser und andererseits durch die Nennbreite determiniert. Der Einfluss des Durchmessers bewirkt, wie in Kapitel 7.9 beschrieben, einen flacheren Funktionsverlauf. Mit Abnahme der Nennbreite verteilt sich aber der Druck zunehmend in Fahrtrichtung. Der Funktionsverlauf in Abbildung 51 für einen Reifen von 1020 mm im Durchmesser und 378 mm in der Nennbreite zeigt nahezu keinerlei Unterschiede zu einem Reifen mit einem Durchmesser von 1810 mm und einer Nennbreite von 800 mm (Abb. 52).

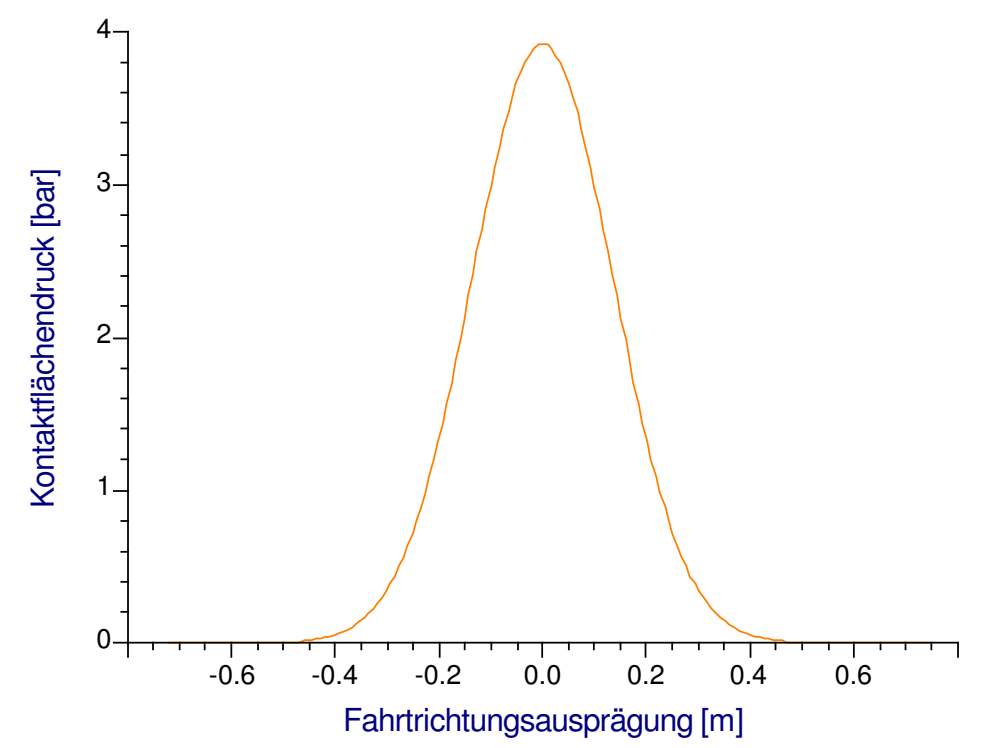

Abbildung 51: Prognose einer Druckverteilungsfunktion in Fahrtrichtung (Radlast 30 kN, Fülldruck 3,0 bar, Nennbreite 378 mm, Durchmesser 1020 mm)

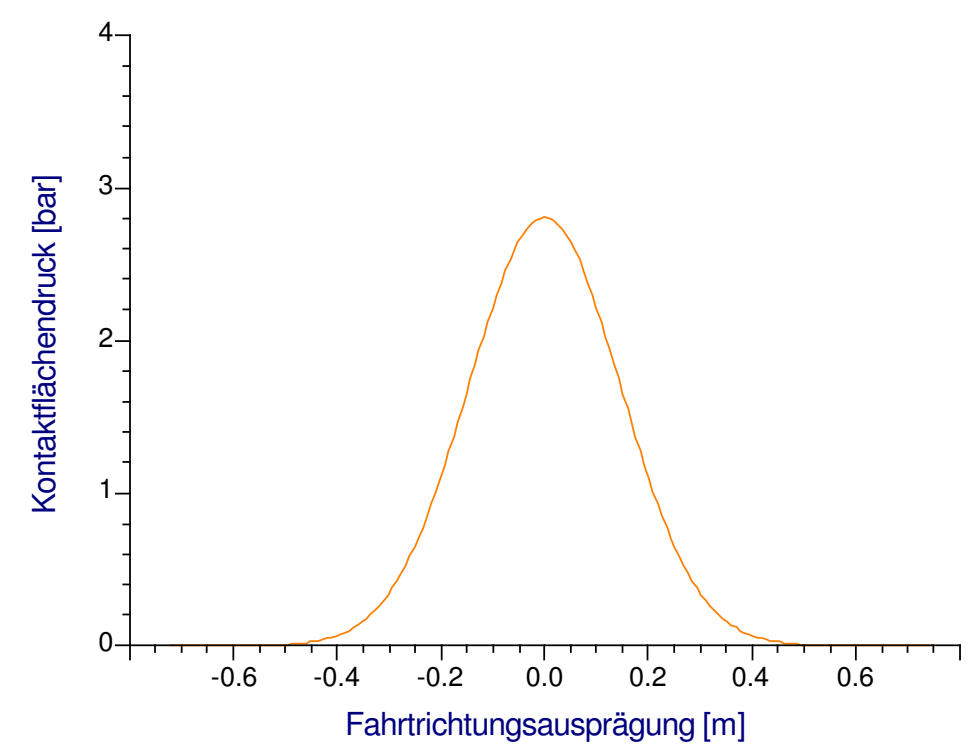

Abbildung 52: Prognose einer Druckverteilungsfunktion in Fahrtrichtung (Radlast 30 kN, Fülldruck 3,0 bar, Nennbreite 800 mm, Durchmesser $1810 \mathrm{~mm}$ )

Durch die prognostizierten Druckverteilungen wird deutlich, dass Reifen mit größerer Nennbreite und größerem Durchmesser ein geringeres Funktionsmaximum aufweisen. Reifen mit grö- 
Berer Nennbreite bewirken einen flacheren Verlauf der Durckverteilungsfunktion in Breitenausprägung. Der Funktionsverlauf in Fahrtrichtungsausprägung wird mit Zunahme des Durchmessers und mit Abnahme der Nennbreite flacher. Werden die Druckverteilungen aber in Breitenund Fahrtrichtungsausprägung insgesamt beurteilt, so kann grundsätzlich festgestellt werden, dass größere Nennbreiten und größere Durchmesser geringere Druckbelastungen nach sich ziehen.

Die Regressionsgleichungen für die Konstanten $a_{m}, c_{x}$ und $c_{y}$ dürfen auf alle Reifen angewendet werden, deren Parameter innerhalb der Grenzwerte des Reifenkollektivs liegen. Daraus ergibt sich folgender Gültigkeitsbereich:

$\begin{array}{lll}\text { Fülldruck } & : & 0,5 \text { bar bis } 5,0 \text { bar } \\ \text { Radlast } & : & 10 \mathrm{kN} \text { bis } 50 \mathrm{kN} \\ \text { Nennbreiten } & : & 378 \mathrm{~mm} \text { bis } 800 \mathrm{~mm} \\ \text { Durchmesser } & : & 1020 \mathrm{~mm} \text { bis } 1810 \mathrm{~mm}\end{array}$

Die Radlasten müssen grundsätzlich innerhalb der Tragfähigkeitsgrenzen der Reifen liegen.

\subsection{Der mittlere Druck}

Die bis hier vorgestellten Ergebnisse bezogen sich ausschließlich auf die im Rahmen der Untersuchungen gewonnenen Daten zur Maximalverteilung des Druckes. Neben diesen maximalen Drücken erschien es unter bodenökologischen Aspekten ebenfalls sinnvoll, die mittleren Drücke bei Befahrung zu untersuchen.

Über die in Kapitel 7.3 beschriebene Ausgleichsfunktion

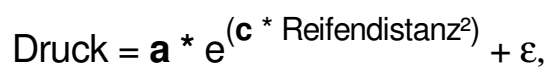

kann man den mittleren Druck eines jeden Reifens über den Mittelwertsatz der Integralrechung nicht arithmetisch bestimmen, da die zugrundeliegende Funktion keine exakt zu umreißende Stammfunktion besitzt.

Der mittlere Druck wird deshalb in separaten Verteilungen aus den in Breiten- bzw. Fahrtrichtung gemittelten Drücken errechnet. Durch Addition der Messwerte pro Zeile und Spalte, dividiert durch die Anzahl der belasteten Zellen wird der jeweilige Druckmittelwert ausgegeben. Nicht belastete Pixel (0-Werte) werden bei diesen Berechnungen ausgeschlossen. Auf Basis dieser Berechnungen geben die einzeln abgetragenen Mittelwerte den Verlauf des jeweiligen Kontaktflächendruckes in Breiten- und Fahrtrichtung wieder. In Abbildung 53 ist exemplarisch der Druckverlauf in Breitenrichtung für den Reifen T22 (750/45-30.5 169/A8 428) bei einer Radlast von $30 \mathrm{kN}$ und einem Fülldruck von 3,0 bar dargestellt. 


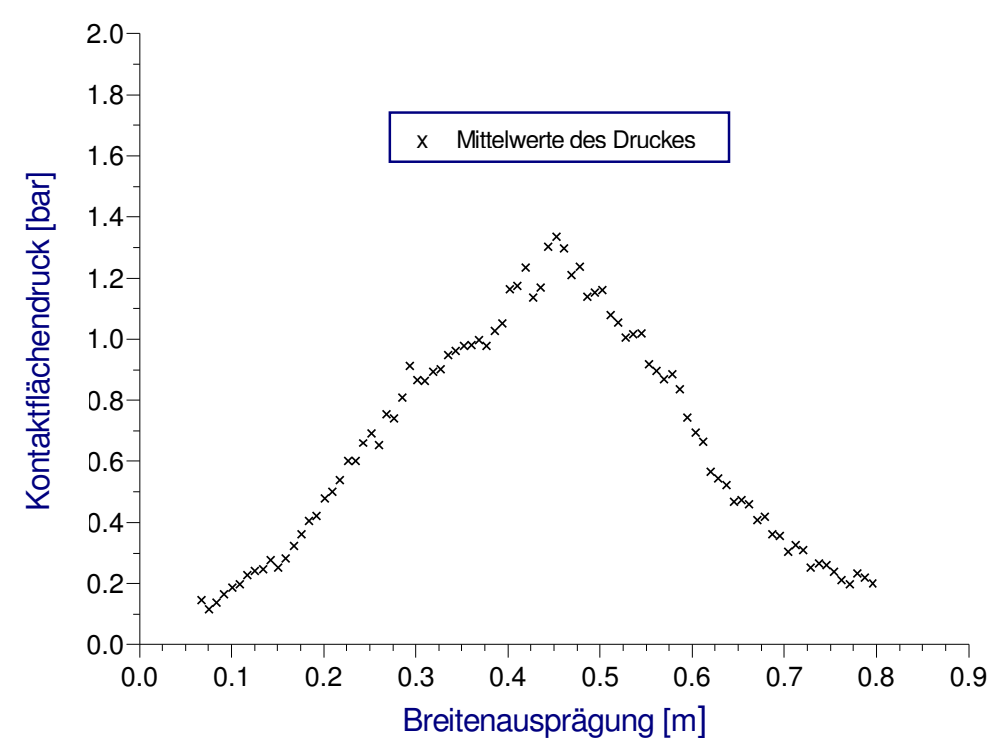

Abbildung 53: Verlauf des mittleren Kontaktflächendruckes in Breitenrichtung (Reifen T22, Radlast 30 kN, Fülldruck 3,0 bar)

Der Verlauf der Mittelwerte ähnelt denen der Maximalwerte. Deshalb erfolgt die Anpassung der Funktion nach dem im Kapitel 7.3 beschriebenen Regressionsmodell und ergibt sowohl für die Betrachtung der Druckverläufe in Breiten- als auch in Fahrtrichtung zufriedenstellende Bestimmtheitsmaße, die sich im Durchschnitt bei $93 \%$ bewegen. Für Breiten- und Fahrtrichtung werden geringe Standardabweichungen von $0,043 \%$ und $0,039 \%$ ausgegeben.

Abbildung 54 zeigt den Ausgleich des mittleren Druckes für den Reifen T22 bei der Radlast von $30 \mathrm{kN}$ und einem Fülldruck von 3,0 bar .

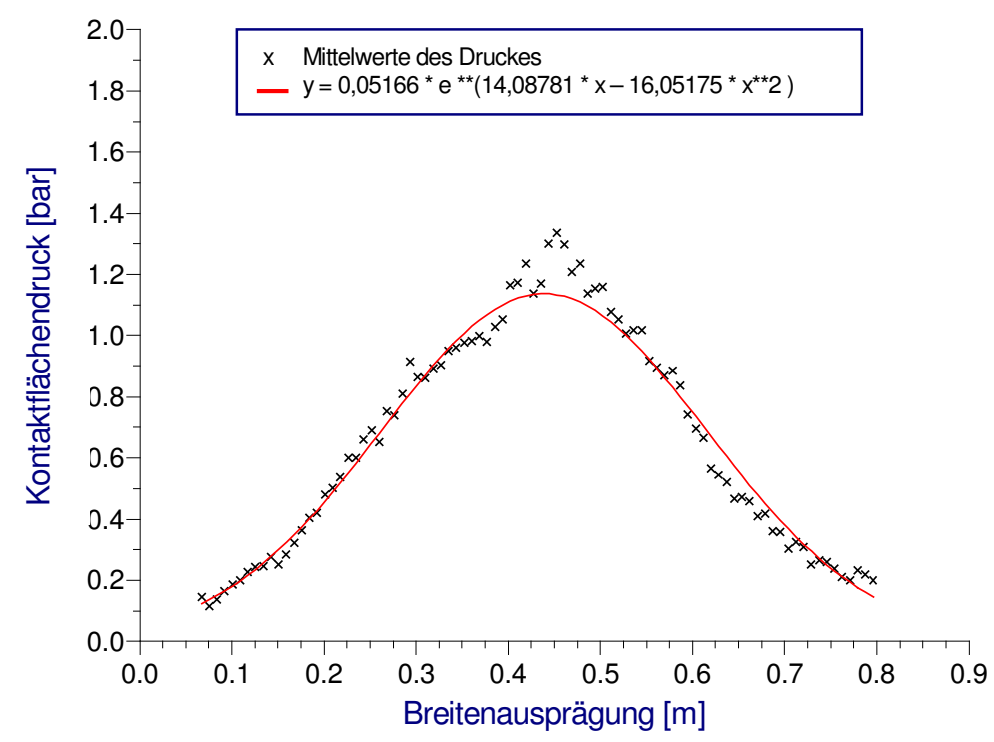

Abbildung 54: Ausgleich des mittleren Kontaktflächendruckes in Breitenrichtung (Reifen T22, Radlast 30 kN, Fülldruck 3,0 bar) 
Werden die jeweiligen Ausgleiche für die Mittel- und Maximalwerte zusammen in einer Abbildung abgetragen, so zeigen diese an den Rändern annähernd konforme Verläufe. Zur Mitte hin ändert sich dieses Verhältnis, so dass im Zentrum der Aufstandsbreite der maximale Druck einem Vielfachen des mittleren Drucks entspricht (Abb. 55).

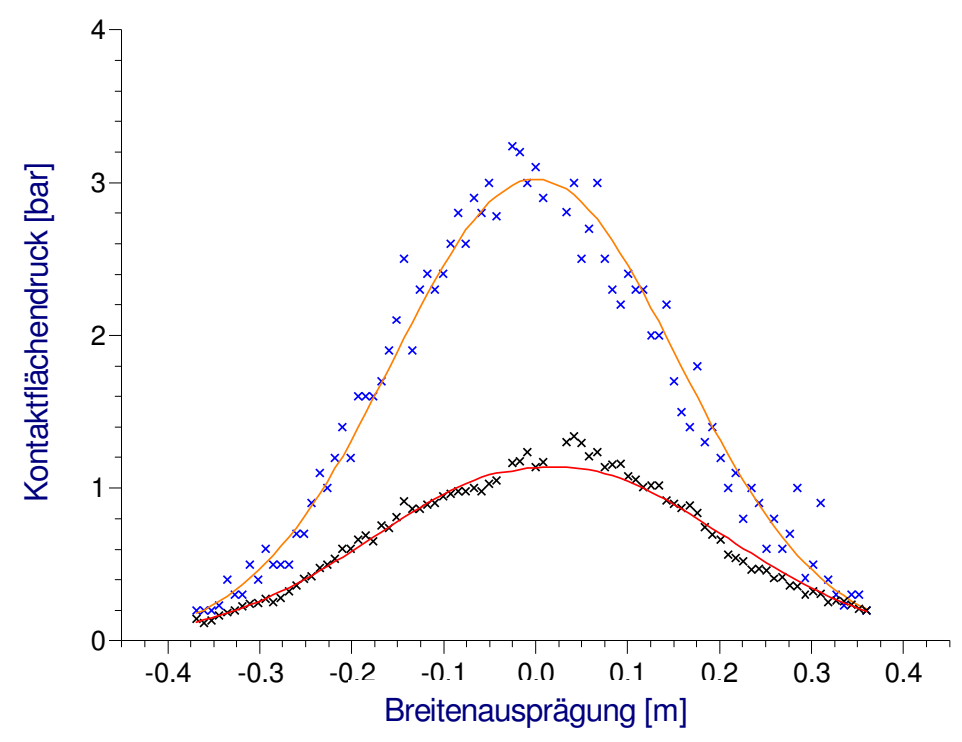

Abbildung 55: Ausgleich des mittleren und maximalen Kontaktflächendruckes in Breitenrichtung (Reifen T22, Radlast 30 kN, Fülldruck 3,0 bar)

Das Maximum der ausgeglichenen maximalen und mittleren Drücke in Breiten- und Fahrtrichtung wird durch die Konstante a ausgedrückt.

Die Druckmaxima in Breiten- und Fahrtrichtung, respektive die Konstanten $a_{x}$ und $a_{y}$, werden wegen sehr geringer ausgleichsbedingter Abweichungen zu einem Mittelwert $a_{m}$ und $a_{m e a n}$ für die Maximalwert- und Mittelwertfunktionen zusammengefasst.

Zwischen $a_{m}$ und $a_{\text {mean }}$ besteht ein plausibler, enger Zusammenhang: der Korrelationskoeffizient beträgt 0,932 und gilt als hochsignifikant gesichert. Die Streuwolke, die sich ergibt, wenn beide Drücke gegeneinander abgetragen werden, zeigt Abbildung 56. 


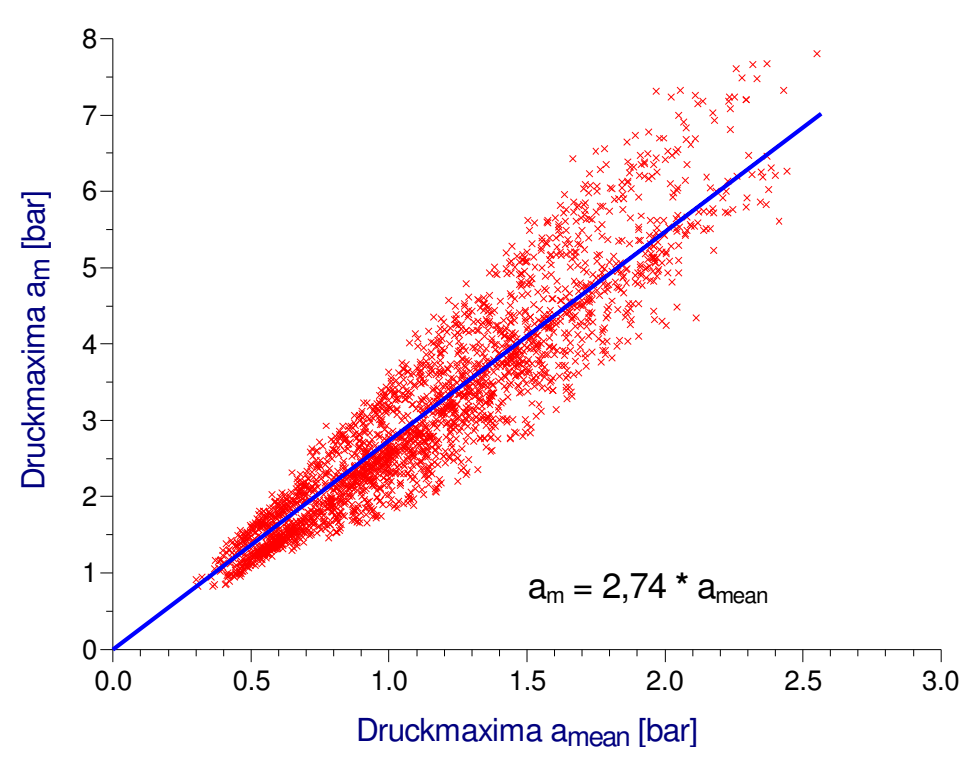

Abbildung 56: Relation zwischen $a_{m}$ und $a_{\text {mean }}$ mit eingezeichneter Ausgleichsgeraden

Zur Überprüfung dieses Zusammenhanges wurde eine durch den Ursprung gezwungene Einfachregression gerechnet und in Abbildung 56 durch eine blaue Ausgleichsgerade dargestellt. Dabei bildete $a_{m}$ die Zielgröße und $a_{\text {mean }}$ die unabhängige Variable. Mit einer ausreichenden statistischen Sicherheit (korrigiertes Bestimmtheitsmaß $98 \%$ ) lässt sich folgende Regressionsgleichung herleiten:

$$
\mathrm{a}_{\mathrm{m}}=\mathrm{k}^{*} \mathrm{a}_{\text {mean }}+\varepsilon
$$

Dieses Modell bringt den Vorteil, dass man über die Regressionsgleichung zu einem Faktor (k) von 2,74 gelangt, der darüber informiert, um wie viel der Spitzendruck $\left(a_{m}\right)$ über dem mittleren Druck $\left(\mathrm{a}_{\text {mean }}\right)$ liegt.

Durch Umstellung der Regressionsgleichung erlaubt diese bei Kenntnis des maximalen Druckes die Berechnung des annähernden mittleren Druckes:

$$
a_{m e a n}=a_{m} / k-\varepsilon
$$




\section{Diskussion}

\subsection{Marktanalyse, Messtechnik und Datenerhebung}

Das Projekt PrAllCon hatte zum Ziel, die Druckverteilung auf Kontaktflächen unter Forstreifen zu erforschen und Gesetzmäßigkeiten zu formulieren, die es erlauben, solche Druckverteilungen in Zukunft rechnerisch zu prognostizieren. Voraussetzung dafür war, die in der forstlichen Praxis montierten Reifen zu erheben, eine geeignete Messtechnik zur Erfassung der Kontaktflächendruckverteilungen zu konstruieren sowie die gewonnenen Daten statistisch fundiert zu analysieren und zu interpretieren.

Ein entscheidendes Kriterium für die Erforschung der Kontaktflächendruckverteilung war neben der Konstruktion der Messtechnik, ein repräsentatives Reifenkollektiv festzulegen. Hierzu wurde eine Befragung der Forstmaschinenhersteller und Generalimporteure durchgeführt. Bemerkenswert ist, dass die Befragten die gewünschten Informationen nahezu vollständig zur Verfügung stellten und sich daraus deutschlandweit ein für das Jahr 2002 aktueller Bestand an Forstmaschinen und deren Bereifungen ableiten ließ. Das resultierende Bemessungskollektiv von 70 Reifen deckte dabei annähernd die in der deutschen Forstwirtschaft montierten Reifendimensionen ab. Die Reifen wurden vollständig in Form einer unentgeltlichen Sachmittelleistung von der Reifenindustrie gestellt. Die logistische Abwicklung erfolgte dabei nach zu bemessenden Arbeitskapazitäten am IFA und bestimmten zeitlichen Restriktionen der Reifenhersteller.

Bei den zu bemessenden Rädern handelte es sich um solche mit fabrikneuen Reifen, die konstruktionsbedingt andere Eigenschaften aufweisen als Gebrauchtreifen. Dieser Umstand machte es notwendig, eine Maschine zu entwickeln, die die von den Reifenherstellern zur Verfügung gestellten Neureifen vor dem Bemessen standardisiert „walkt“. Allerdings gab es für die zu bewalkenden Neureifen keine eindeutige Definition eines „eingefahrenen Reifens“. Seitens des IFA wurde angesichts fehlender, allgemein verbindlicher Vorgaben eine unter den Simulationsbedingungen "gefahrene" Strecke von 5 km für ein Durchwalken der Karkasse definiert. Die von MATTHIES UND ZIESAK (2001) angeführte Empfehlung, nach der ein Reifen nach fünfzigmaligem Einfedern als bewalkt gilt, wurde als nicht ausreichend angesehen. In Rücksprache mit Forschungs- und Entwicklungsabteilungen der Reifenhersteller ergaben sich erste Anhaltspunkte. Diese hielten ebenfalls eine Strecke von $5 \mathrm{~km}$ für ausreichend, um die Karkasse eines Neureifens soweit anzupassen, dass hinsichtlich einer Bemessung der in der Kontaktfläche herrschenden Drücke keinerlei Unterschiede zu einem Gebrauchtreifen bestehen.

Die technische Funktion des Radlastsimulators und deren Bedienung waren während der gesamten Messphase einwandfrei. Alle 70 Reifen wurden auf weichem Untergrund bemessen. Darüber hinaus wurden für 29 Reifen zusätzliche Messungen auf hartem Untergrund durchgeführt und acht Reifen mit Wasserfüllung auf hartem und weichem Untergrund untersucht. Da die Messungen der mit Wasser befüllten Reifen und die Messungen auf hartem Untergrund in einer separaten Untersuchung ausgewertet wurden, erfolgte in der vorliegenden Arbeit keine nähere Betrachtung. 
Die Entscheidung, das gesamte Reifenkollektiv auf weichem Untergrund zu bemessen entstand aus der Überlegung, dass Bodenstrukturveränderungen maßgeblich durch das Einsinken des Reifens verursacht werden und demzufolge nicht nur die Stollen, sondern der gesamte Kontaktbereich eines Reifens einschließlich der Stollenzwischenräume tragende Funktionen im Waldboden übernimmt. Diese als Ganzes erhobenen Kontaktflächenabdrucke wurden deshalb für die Untersuchung der Verteilung des Druckes herangezogen. Die Ergebnisse einer Vorstudie zeigten, dass ein Sand mit der Korngröße $<1 \mathrm{~mm}$ und einer Füllhöhe von $20 \mathrm{~cm}$ (Sandbox) die besten Resultate in Bezug auf die Wiedergabe der Druckverteilung in der Kontaktfläche erbrachte und die Stollenzwischenräume vollständig ausfüllte. Die auf weichem Untergrund erhobenen Kontaktflächendruckverteilungen wurden im weiteren statistisch analysiert und ausgewertet.

Auch wenn bodenökologische Aspekte den Anstoß für die Analyse der Schnittstelle „ReifenBoden"gaben und die Untersuchungen mit einer $20 \mathrm{~cm}$ hohen Sandauflage auf Rückschlüsse im bodenkundlichen Sinne hindeuten, sei an dieser Stelle betont, dass die Erhebung der Kontaktflächendruckverteilungen im Vordergrund stand und keinerlei Aussagen hinsichtlich bodenstrukturverändernder Auswirkungen in dieser Arbeit getroffen wurden.

Die Tekscan-Sensormatten zur Erhebung der Kontaktflächendruckverteilungen auf weichem Untergrund unterlagen einem dem Versuchsablauf entsprechenden normalen Verschleiß. Als besonders empfindlich erwiesen sich die Sensormattenanschlüsse und die Leiterbahnen. Die zum Einlesen der Daten notwendigen Handles mussten oftmals mehrfach angeschlossen werden. Die Leiterbahnen bedurften beim Positionieren der Sandbox eines zusätzlichen Schutzes in Form eines Silikonpuffers.

Die I-Scan-Software ermöglichte zwar die Aufnahme der Rohdaten, das Laden der entsprechenden Kalibrierung und das Abspeichern im ASCII-Format; dies konnte allerdings nur separat für jede einzelne Sensormatte erfolgen. Eine weitere Auswertung und Analyse der Daten setzte aber voraus, dass die Daten jeder einzelnen Sensormatte (vier bzw. acht bei Überstandsmessungen) zu einer Gesamtdatenmatrix zusammenfügt werden. Die hierzu auf dem Markt zu erhaltenden Softwarelösungen entsprachen nicht der gewünschten Bedienerfreundlichkeit. Deshalb entwickelte das IFA eigene Programme, mit deren Hilfe die geforderten Auswertungsschritte umgesetzt werden konnten. Die erstellte Software des IFA war problemlos für eine weitere Datenauswertung geeignet. Für zukünftige Untersuchungen stehen somit leistungsfähige und benutzerfreundliche Programme zur Verfügung, mit denen von der Erhebung der Daten mit der I-Scan-Software bis zu deren Auswertung mit bereits etablierter statistischer Analysesoftware die Datenverarbeitungskette vervollständigt wird.

Messtechnische Ungenauigkeiten, insbesondere eine Übersteuerung einzelner Sensorzellen und/oder der partielle Ausfall von Sensorreihen und -spalten, die während der starken Beanspruchung der Sensormatten auftraten, machten eine Bearbeitung der Kontaktflächenabdrucke mittels verschiedener Artefaktroutinen erforderlich. Die Eliminierung der Artefakte erfolgte dabei auf gutachtlicher Basis. Durch Mittelung der umliegenden Zellenwerte wurde der Druckwert aktiv verändert. Da aber der für die ausgefallenen bzw. übersteuerten Zellen eingesetzte Druckwert einer Mittelung der realen Messwerte der Nachbarzellen entspricht, ist davon auszugehen, dass dieser theoretisch ermittelte Druck mit dem realen Druck an dieser Stelle annähernd übereinstimmt. Zur zusätzlichen Orientierung dienten eine Legende mit Angabe der 
Druckwerte und bestimmte statistische Parameter, wie die Standardabweichung und der Variationskoeffizient.

\subsection{Auswertung und Analyse der Daten}

Generell konnte bei der ersten Betrachtung der Kontaktflächenabdrucke festgestellt werden, dass sich mit zunehmender Radlast und steigendem Fülldruck der Schwerpunkt der Druckbelastung in der Mitte der Kontaktfläche konzentriert und stetig zur Peripherie hin abnimmt. Die sich anschließenden Auswertungen zeigten, dass der Druck sich durchweg in Form einer Glocke mit elliptischer Querschnittsfläche verteilt.

Bodenökologische Aspekte sprachen dafür, dass für eine weitere Untersuchung die Maximalverteilungen der Drücke heranzuziehen sind. So verweist WEIBBACH (2001a) in einer Untersuchung für landwirtschaftliche Reifen darauf, dass für die Wirkung auf den Boden nicht der mittlere Kontaktflächendruck maßgebend ist, sondern der maximal in einem Punkt der Kontaktfläche auftretende Druck. ZIESAK (2004) kommt ebenfalls zu dem Schluss, dass für die Wirkung auf den Boden die Kenntnis des mittleren, rechnerischen Kontaktflächendruckes allein nicht hilfreich ist, da der an einem Punkt maximal auftretende Druck zum einen vom Mittelwert abweichen kann und zum anderen die Größe einer eventuellen Bodenstrukturveränderung vor allem von diesem auftretenden Maximalwert abhängt.

Die maximalen Druckwerte wurden mittels in Breiten- und Fahrtrichtung vorgenommener Vertikalschnitte durch die Verteilungsglocken ermittelt. Diese glockenförmigen Druckverläufe konnten mit einer Ausgleichsfunktion erfasst werden. Für die beobachteten Maximalwerte aller Reifen wurde eine gute bis sehr gute Anpassung mit dieser Funktion erzielt. Ausgedrückt wird dies u. a. durch die sich im Mittel bewegenden Bestimmtheitsmaße von $92 \%$. Mit dem dargelegten Modellansatz war es somit möglich, jede Kontaktflächendruckverteilung zu beschreiben und gültige Vorhersagen für das gesamte Reifenkollektiv zu entwickeln.

Der Verlauf der Funktion wird maßgeblich durch die Konstanten $\mathrm{a}, \mathrm{b}$ und $\mathrm{c}$ beeinflusst.

$$
y=a \cdot e^{b \cdot x+c \cdot x^{2}}
$$

Die Lage innerhalb des Koordinatensystems wird durch die Konstante $b$ bestimmt. Das Maximum der Ausgleichsfunktion ist aber nicht immer identisch mit dem Zentrum der Messmatrix. Eine Verschiebung des Koordinatenursprungs ins Funktionsmaximum führte zur Eliminierung der Konstanten $b$ und zur Gleichsetzung des Wertes der Konstanten a mit dem Maximum der jeweiligen Druckverteilung. Die Werte für $\mathrm{c}$ sind durchweg negativ. Sofern die Konstante a nicht variiert, bewirkt ein größerer (absolut gesehen kleinerer) c-Wert ein Flacherwerden der Glockenkurve. Die Verkürzung der Ausgleichsfunktion um die Konstante b ermöglichte eine einfachere Handhabung und erlaubte es zudem, die Druckverteilung eines jeden Reifens mit nur zwei Konstanten dieser Modellgleichung zu beschreiben. 


$$
y=a \cdot e^{c \cdot x^{2}}
$$

Multiple Regressionsanalysen sollten Aufschluss darüber geben, welche Variablen die einzelnen Konstanten und damit den Verlauf der Druckverteilungsfunktionen beeinflussen.

Die Konstante a wurde in Breiten- und Fahrtrichtung zu einem Mittelwert $a_{m}$ zusammengefasst, während die Konstante $c$ weiterhin für beide Richtungen separat untersucht wurde.

Die Regressionsergebnisse machten deutlich, dass die Konstante $a_{m}$ neben Radlast und Fülldruck durch die Reifenparameter Nennbreite und Durchmesser beeinflusst wird. Werden an dieser Stelle die Variablen näher betrachtet, so kann konstatiert werden, dass die Variablen Radlast und Fülldruck die dominierenden Größen sind, die mit einem Anteil von 76,3\% von insgesamt 78,2 \% der erklärten Streuung den Spitzendruck beeinflussen. Der Einfluss der im vierten Modell aufgenommenen Variablen Durchmesser mit einem Anteil von 0,4\% an der erklärten Streuung ist deshalb durchaus kritisch zu bewerten. Allerdings sprachen Praktikabilitätsgründe für die Aufnahme, denn die Einbeziehung von Variablen obliegt dem Untersuchenden, von dessen Erfahrung und Interpretation die Auswahl eines Modells, von dem er annimmt, dass es sein Datenmaterial gut abbildet, abhängt (s. hierzu JACKE, 1980; BACKHAUS ET AL., 2000). Ein größerer Reifendurchmesser hat eine direkte Wirkung auf die Länge des Kontaktflächenabdruckes. Es ist zu vermuten, dass sich die Kräfte so auf eine größere Aufstandsfläche verteilen können und damit zur Verringerung des Kontaktflächendrucks beitragen. Mit den vier berücksichtigten Variablen gelingt es, $78 \%$ der Streuung der Zielgröße und damit die Beeinflussung des Spitzendruckes zu erklären. Werden die Regressionskoeffizienten der einzelnen unabhängigen Variablen betrachtet, so steigert jede zusätzliche Erhöhung der Radlast um 10 kN (oder von einer Tonne) den Spitzendruck um 0,0726 bar, jedes bar an zusätzlichem Reifenfülldruck sogar um 0,4413 bar. Wenn man die Nennbreite und den Durchmesser eines Reifens um 100 mm steigert, mindert sich der Druck um 0,0017 bar bzw. um 0,0004 bar. Damit wird deutlich, dass der Fülldruck enormen Einfluss auf die Zielgröße $a_{m}$ und damit auf den Spitzendruck besitzt.

Die für einen flacher- oder steilerwerdenden Funktionsverlauf in Breitenrichtung verantwortliche Konstante $c_{x}$ wird maßgeblich durch die Variablen Nennbreite, Radlast und Fülldruck beeinflusst. Die Reihenfolge der aufgenommenen Variablen lässt erkennen, dass die Nennbreite mit einem Anteil von 38,1\% am Gesamtbestimmtheitsmaß den größten Einfluss auf die Breitenausprägung besitzt. Einen weiteren Erklärungsbeitrag von $21,9 \%$ und 9,9\% liefern die Variablen Fülldruck und Radlast. Hingegen beeinflussen die Variablen Radlast, Fülldruck, Durchmesser und Nennbreite die Konstante $c_{y}$ und damit die Fahrtrichtungsausprägung. Allein Radlast und Fülldruck mit Anteilen von 32,5 \% und 27,7 \% erklären 60,2 \% der Streuung in Fahrtrichtungsausprägung. Die Variablen Durchmesser und Nennbreite fügen ein weiteres Erklärungspotential von 10,1\% hinzu. Mit den berücksichtigten Variablen lassen sich jeweils $70 \%$ der Streuung der Zielgrößen $c_{x}$ und $c_{y}$ erklären und damit die Beeinflussung der Breitenund Fahrtrichtungsausprägung.

Werden an dieser Stelle die Vorzeichen der Regressionskoeffizienten der einzelnen Variablen betrachtet, so zeigt sich für die Breitenausprägung, dass der Funktionsverlauf bei Zunahme der Nennbreite und einer Erhöhung der Radlast flacher, bei Steigerung des Fülldruckes steiler 
wird. Determiniert die Nennbreite den Funktionsverlauf in Breitenrichtung, deuten sachlogische Überlegungen darauf hin, dass der Funktionsverlauf in Fahrtrichtung neben Radlast und Fülldruck durch den Durchmesser beeinflusst wird. Darüber hinaus wirkt sich aber auch die Nennbreite aus. Der Einfluss wird deutlich, wenn die einzelnen Variablen näher betrachtet werden: Die Druckprofilausprägung wird in Fahrtrichtung länger bei höheren Radlasten und bei größeren Raddurchmessern, kürzer bei steigenden Fülldrücken und bei wachsenden Nennbreiten. Der Einfluss der Nennbreite erklärt sich hierbei folgendermaßen: Je schmaler ein Rad bzw. ein Reifen ist, desto eher wird sich bei einem gegebenen Druck dieser auf "mehr Fläche“ in Fahrtrichtung verteilen. Ein Einfluss des Durchmessers in Breitenausprägung konnte dagegen nicht festgestellt werden. JACKE (2005b) verweist darauf, dass die Aufdruckfläche der Niederquerschnittreifen im großen Mittel nahezu kreisförmig ist, während die Standardreifen die Ellipsenachse in Fahrtrichtung fast das 1,5fache der Achse in Breitenausprägung beträgt. Niederquerschnittreifen verteilen die Radlast weitgehend gleichmäßig auf die Fahrtrichtung und die Breite, während Standardreifen weniger die Breite als vielmehr die Längenausprägung (in Fahrtrichtung) nutzen.

Interessant an dieser Stelle ist der Einfluss des Fülldruckes, der mit Anteilen von 21,9 \% und 27,7 \% die Streuung der Zielgrößen $c_{x}$ und cy erklärt. Die negativen Vorzeichen der Regressionskoeffizienten der Variablen Fülldruck verweisen eindeutig darauf, dass mit der Abnahme die Werte für $c_{x}$ und $c_{y}$ größer, der Verlauf einer Druckverteilungsfunktion insgesamt flacher wird. Es besteht demnach eine enge Beziehung zwischen dem Reifeninnendruck und dem Kontaktflächendruck, d. h. mit einem geringeren Fülldruck sinkt auch der Kontaktflächendruck, da die Auflast des Rades auf eine größere Fläche verteilt werden kann. Wie bei den Ergebnissen zur Untersuchung der Zielgröße $a_{m}$ festgestellt, wird mit einer Verringerung des Fülldruckes auch der Spitzendruck maßgeblich beeinflusst und sorgt ebenfalls für eine niedrigere Druckbelastung des Untergrundes. Das Potential einer Verringerung des Reifeninnendruckes ist demzufolge evident.

Die Bedeutung eines geringen Fülldruckes, um die Bodenbelastung zu verringern ist seit längerem bekannt. So ist es nicht verwunderlich, dass sowohl in der Forstwirtschaft als auch in der Landwirtschaft zahlreiche Untersuchungen durchgeführt wurden, die das Potential eines verminderten Fülldruckes anführten. Aus dem bereits umfangreichen Schrifttum sind z. B. zu nennen: RENIUS (1985); BACKHAUS (1993, 1994, 1998); SCHWANGHART (1993); SEMMEL UND HORN (1993); ISENSEE UND WEIBBACH (1994); DISERENS ET AL. (2002); DÖLL (1998); GYSI UND GRUNDER (1999); JACKE (1999); HAUCK (2001); PÜTZ (2001); MATTHIES UND ZIESAK (2001); SCHLOTTER UND KUTZBACH (2001); WEIBBACH (2001a, 2001b); DISERENS (2002); WEISE (2002, 2003). Dabei kommen alle Autoren in ihren Untersuchungen zu ähnlichen Ergebnissen: Als Hauptaussage wird festgestellt, dass durch eine Verringerung des Luftdruckes der Reifen stärker einfedert und sich demzufolge die Radlast auf eine größere Fläche verteilt, wodurch der Kontaktflächendruck und damit die Bodenbelastung abnimmt. In diesem Zusammenhang werden als weitere Vorteile einer Reifeninnendruckabsenkung die verminderte Bodenverdichtung, geringere Fahrspurtiefen, reduzierter Rollwiderstand, verbesserte Zugkraftübertragung und günstigerer Fahrwirkungsgrad bei weniger Schlupf, geringerer Kraftstoffverbrauch, höherer Fahrkomfort infolge einer Schwingungsentlastung für den Fahrzeugführer, geringere Wetterabhängigkeit und verbesserte Selbstreinigung der Reifen genannt. 
Eine Verringerung des Fülldruckes ist maßgeblich von der Tragfähigkeit des Reifens abhängig. Dabei muss die Tragfähigkeit so groß sein, dass sie die Fahrzeugmasse und die Zuladung sowie auftretende Achslaständerungen ohne Reifenschäden aufnehmen kann (SCHULZ, 1994). Die Stabilität eines Reifens wird maßgeblich durch die Karkasse geprägt. Die Karkassenstabilität wird heutzutage vorwiegend durch den Load-Index (LI/Tragfähigkeitskennzahl) angegeben (s. Kap. 1.3). Der LI ist eine ingenieur-mathematische Verknüpfung einer Zahlenfolge mit einer tolerierten Radlast (Anhang 1, Abb. 1.1). Da die tolerierbare Last mit der Drehzahl des Rades sinkt, muss sie praktikabel an Geschwindigkeitsgrenzen (Speed-Index) gekoppelt werden. Dieser Speed-Index (SI) weist durch Verknüpfung von Buchstaben (aufsteigend sortiert in alphabetischer Folge) Richtgeschwindigkeiten aus (Anhang 1, Abb. 1.2). Das Gros der im forstund landwirtschaftlichen Bereich eingesetzten Reifen orientieren sich am SI „A8“, der eine Geschwindigkeit von $40 \mathrm{~km} / \mathrm{h}$ erlaubt.

Nach RENIUS (1985) und SCHULZ (1994) ist die Reifentragfähigkeit neben der Tragfähigkeitskennzahl und der Fahrgeschwindigkeit auch von der Reifengröße (Luftvolumen) und dem Reifenfülldruck abhängig. Dabei steigt die Tragfähigkeit eines Reifens mit dem Reifeninnendruck an und sinkt mit zunehmender Fahrgeschwindigkeit. Die zulässigen Werte der maximalen Last in Abhängigkeit von Innendruck und Geschwindigkeit werden von den Herstellern in Tabellen angeben. KRIEGER (2004) verweist darauf, dass bei einem größeren Reifenvolumen weniger Fülldruck erforderlich ist, um eine bestimmte Tragfähigkeit zu erreichen. Ein größeres Volumen und eine geringe Fahrgeschwindigkeit erlauben nach BACKHAUS (1994) auch eine Absenkung des Reifeninnendruckes. Die Entwicklung im Bereich der Landwirtschaftsreifen (AS-Reifen) tendiert in Richtung zunehmender Volumina. So werden mittlerweile Hinterräder von Ackerschleppern mit vormaligen 38-Zoll-Felgen verstärkt durch Räder mit 32-Zoll-Felgen ersetzt. Bei gleichbleibenden Reifendimensionen ist damit eine Vergrößerung des Volumens verbunden, die einen niedrigeren Fülldruck zu fahren erlaubt. Hiermit soll eine größere Reifenverformung erreicht werden, um eine geringere Bodenbelastung zu erzielen.

Die Tragfähigkeit des Reifens steht, wie bereits erwähnt, bei einem bestimmten Reifeninnendruck in enger Beziehung zur Geschwindigkeit. Mit steigender Geschwindigkeit nimmt die Tragfähigkeit ab. Zurzeit ist davon auszugehen, dass die für Umsetzfahrten auf öffentlichen Straßen und Wegen erforderlichen höheren Reifenfülldrücke (3,0 - 5,0 bar) auch im Wald eingestellt bleiben, da keine Möglichkeit besteht, den Reifendruck in vertretbarer Zeit anzupassen. Abhilfe könnten Reifendruckregelanlagen (CTIS = Central-Tyre-Inflation-System) schaffen. Diese ermöglichen mit akzeptablem Zeit- und Kostenaufwand zu den jeweiligen Arbeitsbedingungen stets mit dem optimalen Reifenluftdruck zu fahren, und zwar mit geringem Fülldruck bodenschonend im Wald/Feld und mit hohem Füldruck reifenschonend auf der öffentlichen Straße. BACKHAUS (1998) verweist darauf, dass solche Systeme vor allem in der Landwirtschaft innerhalb von wenigen Jahren Verwendung gefunden haben. Versuche im forstwirtschaftlichen Bereich wurden u. a. von BACKHAUS (1992, 1993, 1994, 1998) und ARNOLD (2002) durchgeführt.

Die Anpassung des Reifenluftdruckes an die sich ändernden Arbeitsbedingungen ist eine der wesentlichen Möglichkeiten, das Betriebsverhalten der Forstmaschinen zu verbessern. Allgemein gilt es, je nach Radlast, Geschwindigkeit und Zugkraftbedarf, den geringst möglichen Fülldruck einzustellen, ohne dass die Bereifung hierdurch langfristig beschädigt wird. 
Dabei kann festgestellt werden, dass in der Landwirtschaft durchweg niedrigere Fülldrücke gefahren werden als in der Forstwirtschaft. Nach ISENSEE UND WEIBBACH (1994) erlauben Breit- und Zwillingsbereifungen den Reifeninnendruck auf unter 1,0 bar abzusenken. PÜTZ (2001) empfiehlt zwei Faustzahlen: So sollte der Reifeninnendruck bei Pflege- und Bestellarbeiten und für Grünland nicht mehr als 1 bar, bei Erntearbeiten nicht mehr als 2,0 bar übersteigen. DISERENS (2004) verweist darauf, dass seit Mitte der 1980er Jahre die Mechanisierung in der Landwirtschaft außerordentlich stark zugenommen hat. Dabei ist nicht nur die Leistungsfähigkeit der Maschinen kontinuierlich gestiegen, sondern parallel dazu auch deren Maschinenmassen. Mit Radlasten bis über $110 \mathrm{kN}$ und Maximalmassen um $60 \mathrm{t}$ sind etwa in der Zuckerrübenernte die bisher schwersten Landmaschinen im Einsatz. ISENSEE UND WEIBBACH (1994) geben für eine Maschine mit Bunkerinhalt, die in der Zuckerrübenernte 40 t erreicht, einen Reifenfülldruck von 1,6 bar an.

Der Trend der in der Forstwirtschaft eingesetzten Maschinen hinsichtlich ihrer Leistungssteigerung und der damit einhergehenden Zunahme der Gesamtmassen ( $5 \mathrm{t}$ bis $30 \mathrm{t}$ ) vollzog sich parallel zu der in der Landwirtschaft stattfindenden Entwicklung. Allerdings finden sich in der Forstwirtschaft noch wesentlich höhere Reifenfülldrücke. Die meisten Forstmaschinen (Harvester, Forwarder, Forstschlepper) fahren trotz Niederquerschnittbereifung mit einem Reifenluftdruck von durchschnittlich 3,0 - 5,0 bar, auch unter Hinweis der aktuellen Tragfähigkeitswerte der Reifenhersteller. Es werden demnach die Vorteile dieser Spezialbereifung in der Forstpraxis vielfach nicht voll genutzt.

Die Reifenhersteller begründeten die hohen Fülldrücke mit dem Hinweis auf die im Forsteinsatz bestehenden erheblichen Belastungen durch die Zugkraft der Maschine und die enormen Seitenkräfte, besonders in schwierigem Gelände. Hierdurch wachse die Gefahr von Ventilabrissen durch Verdrehen der Felge im Reifen oder von Schlauchschäden, wodurch unnötige Stillstandzeiten eintreten. Laut JACKE (1999) und HAUCK (2001) bewegen sich deshalb die Hersteller, um Reklamationen zu vermeiden, mit ihren Empfehlungen im sicheren, hohen Bereich, der für höhere Geschwindigkeiten und zum Umsetzen bei Straßenfahrten erforderlich ist.

Untersuchungen von BACKHAUS (1998) haben gezeigt, dass für einen Forstschlepper MB-trac 900 Turbo F (Niederquerschnittbreifung mit Schläuchen - 500/60-26.5 12 PR) der Nennfülldruck von 2,4 bar - für Fahrten auf der Straße - um bis zu 1,2 bar bei der Holzbringung abgesenkt werden kann. Verfügt der Reifen über eine Sicherheitsfelge, so sind Transportfahrten mit einem Reifeninnendruck von 0,6 bar möglich. ARNOLD (2002) vertritt die Auffassung, dass die Zeit der werkseitig vorgeschriebenen Reifeninnendrücke von 3,0 bar und mehr wahrscheinlich vorbei sei. So seien in Zukunft Fülldrücke von 1,5 bar realistisch, denn die heute vorhandenen Reifen halten schon wesentlich mehr aus, als die Hersteller behaupten.

Mittlerweile haben die Reifenhersteller reagiert und in Abhängigkeit von der Bereifung und den Geländebedingungen deutlich niedrigere Grenzwerte für Fülldrücke festgelegt. Grundsätzlich werden die im Rahmen des PrAllCon-Projekts untersuchten Forstreifen in diagonaler Bauweise mit höheren Fülldrücken (3,0 - 5,0 bar) gefahren. Im Gegensatz dazu ist bei den radial gebauten Reifen (Nokian, Michelin) nur eine Befüllung bis maximal 2,4 bar vorgesehen. Zudem kann der Fülldruck der Radialreifen flexibel hinsichtlich variierender Lasten gewählt werden. Bei den Diagonalreifen bieten Trelleborg, Firestone und Barum ebenfalls entsprechende Variationsmöglichkeiten, die auch Fülldrücke von unter 2,0 bar bzw. 1,0 bar (Barum) erlauben. Die 
Spannweiten der von Trelleborg angegebenen Fülldücke reichen von 1,0 - 4,0 bar. Nokian hingegen beschränkt sich überwiegend auf die Vorgabe nur eines Fülldruckes. Dabei reicht die Spannweite der Fülldrücke für die Reifen von 2,4 - 5,0 bar.

Es steht außer Frage, dass die Reifenkonstruktion, die Radlast und der Luftdruck aufeinander abzustimmen sind. Dabei sollte der Reifen mit einem Fülldruck gefahren werden, der die größte, gleichmäßig beanspruchte Aufstandsfläche - bei Einhaltung der Belastbarkeitsgrenzwerte erzielt.

SCHLOTTER UND KUTZBACH (2001) verweisen darauf, dass auf fester Fahrbahn die Kontaktfläche maßgeblich von der Reifeneinfederung abhängt. Mit steigender Radlast nimmt die Einfederung des Reifens zu, wobei eine zunehmende Abplattung der Aufstandsfläche stattfindet. Auf nachgiebigeren Boden wird der Reifen geringer verformt. Nach JACKE (2005a) verhält sich ein mit Luft befültter Reifen auf hartem Untergrund bis zum Erreichen einer gewissen Grenzlast wie eine zusammendrückbare Schrauben- oder Tellerfeder. Der Verlust an Reifenhöhe wächst dabei linear-proportional zur Auflast, oder - aus der umgekehrten Perspektive - die Resthöhe sinkt linear-proportional zur Auflast. Das Zusammenspiel von Reifenkonstruktion und eingebrachtem Fülldruck kann mittels einer Federkonstanten ${ }^{29}$ beschrieben werden. Allgemein gilt, dass eine absolut größere Federkonstante einem härteren oder auch in sich steiferen Reifen entspricht. Laut JACKE (2005a) bestimmen der Fülldruck, dann die Karkassenstabilität (ausgedrückt über den Load-Index) die Elastizität eines Reifens. Auch Schwieger (1996) kommt zu dem Ergebnis, dass die Elastizität des Reifens durch den Luftdruck sowie der Bauart der Karkasse bestimmt wird. Offenbar sind breitere Reifen generell auf Kompensation höherer Auflasten ausgelegt. WEIBBACH (2001a) stellte ebenfalls fest, dass sich mit steigender Reifenbreite die Tragfähigkeit erhöht. Demnach kann bei gleicher Radlast und breiter werdendem Reifen der Luftdruck abgesenkt werden. MATTHIES UND ZIESAK (2001) erklären über ein von innen entwickeltes Druckzonenmodell eine Reifeninnendruckabsenkung bei größeren Reifenbreiten. Des Weiteren verweisen sie darauf, dass der Reifenfülldruck auch mit Anstieg der Tragfähigkeit (höherer Load-Index) stärker abgesenkt werden kann.

JACKE (2005b) stellte bei den auf hartem Untergrund bemessenen Reifen des PrAllConProjekts fest, dass bei niedrigeren Fülldrücken und erhöhten Auflasten an den Flankenrändern deutlich höhere Drücke herrschen. Dabei kann die Belastung der Flanken fast dreimal so hoch ausfallen, wie im Zentrum der Lauffläche. Über die Entwicklung einer Plandruckgeraden wird in Abhängigkeit der Auflast-Fülldruck-Kombination gezeigt, ab wann der Reifen einigermaßen gleichmäßig bedrückt auf die Stahlplatte gebracht wird. Der aus der Plandruckgeraden abgeleitete Plandruckkoeffizient informiert darüber, wie sensibel der Reifen in puncto Fülldruckerhöhung reagiert, um bei gegebener Auflast den Plandruck zu erreichen. Standardreifen gelten dabei durchweg als elastischer (weicher) ausgelegt. Sie federn stärker ein als die Niederquerschnittreifen. JACKE (2005b) folgert daraus: Je härter (weniger elastisch) ein Reifen ausgelegt ist, desto geringer der Mindestfülldruck, der zur Planauflage des Reifens bei gegebener Auflast notwendig ist. Der Kontaktflächendruckabfall zur Reifenmitte oder auch die überproportionale Belastung der Flanken bei zu geringen Fülldrücken führt zu einem erhöhten Verschleiß des

29 Die Federkonstante korrelliert als Proportionalitätsfaktor die Auslenkung einer linearen Feder mit der daraus resultierenden Zug- oder Druckkraft. Nach dem Hook'schen Gesetz ist die rücktreibende Kraft einer Feder proportional zur Auslenkung. 
Reifens, insbesondere beim Betrieb auf harten Fahrbahnen. Der Verschleiß konzentriert sich dabei nicht auf die vornehmlich stützende Flankenpartie, sondern auf das (durch den Druck gering belastete) Zentrum. Dort kommt es im Kontaktflächenbereich zu einer permanenten und systematischen Verformung, die relativ rasch zur Zerstörung der Karkassenschichten führen kann. Aus diesen Gründen geben die Reifenhersteller Mindestfülldrücke an, die sich offensichtlich am Plandruck der Reifen orientieren. Nach JACKE (2005b) sind sie in der teilweise beachtlichen Höhe (fast 4,0 bar) vollends gerechtfertigt, wenn man für Fahrten auf befestigten Wegen und Straßen einer raschen Zerstörung der Reifen vorbeugen will.

Auf weichem Untergrund hingegen ist das Verhalten eines Reifens anders zu bewerten. Nach SCHULZ (1995) ist der Verschleiß im Gelände bedeutend geringer, denn dort sinken die Stollen mehr oder weniger tief ein und werden seitlich abgestützt. Für die Bemessung der Reifen auf weichem Untergrund konnte nur bei hohen Radlasten von $40 \mathrm{kN}$ und $50 \mathrm{kN}$ und geringen Fülldrücken von 0,5 bar und 1,0 bar ein Flankeneffekt in Ansätzen beobachtet werden. Deshalb ist zu vermuten, dass Reifen sich auf nachgiebigerem Untergrund weniger verformen. Zu gleichen Erkenntnissen kamen SCHWIEGER (1996) sowie SCHLOTTER UND KUTZBACH (2001) im Rahmen von Untersuchungen für landwirtschaftliche Reifen. Da die Reifen im Wald mit geringeren Geschwindigkeiten gefahren werden, ist eine Absenkung des Reifeninnendruckes (in Anlehnung an die Herstellerangaben) möglich, ohne Reifenschäden davonzutragen.

An dieser Stelle kann konstatiert werden, dass dem Reifeninnendruck eine zentrale Rolle zukommt und er nicht nur entscheidenden Einfluss auf die Kontaktflächendruckverteilung besitzt, sondern auch in engem Zusammenhang mit konstruktionsbedingten Parametern des Reifens steht.

In der Vergangenheit wurde häufig die Frage diskutiert, ob Niederquerschnittreifen wirklich „besser"als ihre Pendants, die Standardreifen sind (s. hierzu TÜRCKHEIM, 1983; RENIUS, 1985; HOFMANN UND BECKER, 1990; ISENSEE UND WEIBBACH, 1994; SCHULZ, 1994; REINHARDT, 1995). Ferner interessierten zudem die Auswirkungen der Bauweise von Reifen (diagonal/radial) oder der Profilierung (Traktions-/Softprofil). Die Variablen Reifenart, Bauweise und Profilierung wurden in der beschriebenen Regression als Dummies angeboten. Ihr Erklärungsgehalt für die Zielgrößen $a_{m}, c_{x}$ und $c_{y}$ war allerdings so gering, dass sie in den Regressionsmodellen nicht weiter berücksichtigt wurden. Demnach sind weder Unterschiede zwischen Niederquerschnitt- und Standardreifen noch bei Bauweise und Profilierung hinsichtlich ihrer Druckverteilung auf weichem Untergrund nachzuweisen.

\subsection{Prognosemodell und Ausblick}

Für die Druckverteilung auf Kontaktflächen unter Forstreifen kann anhand der vorliegenden Studie insgesamt festgestellt werden, dass diese ausschließlich durch die Variablen Radlast, Fülldruck, Nennbreite und Durchmesser beeinflusst werden.

Neben den maximalen Drücken erschien es ebenfalls sinnvoll, die mittleren Drücke bei Befahrung zu untersuchen. Der Verlauf der Mittelwerte ähnelt denen der Maximalwerte, so dass es nahe lag, die Ausgleichsfunktion, die bereits für die Anpassung der maximalen Druckverläufe geeignet war, auch zur Anpassung der mittleren Druckverläufe anzuwenden. Die durchschnitt- 
lichen Bestimmtheitsmaße lagen mit 93 \% sogar um einen Prozentpunkt höher als die Bestimmtheitsmaße der maximalen Druckverteilungsfunktionen. Unter bodenökologischen Gesichtspunkten stellt die Kenntnis des mittleren Druckes, der für die gesamte Aufstandsfläche als Maßstab genommen werden kann, ein weiteres Bewertungskriterium dar.

Zwischen den Maximaldrücken und den Maxima dieser gemittelten Druckverteilungen bestand eine plausible Korrelation, die sich mit einer Einfachregression herleiten ließ. Es wurde ein Faktor von 2,74 abgeleitet, mit dem bei Kenntnis des maximalen Druckes auf den mittleren Druck geschlossen werden kann.

Werden die Werte, der über die Regressionsgleichungen ermittelten Konstanten $a_{m}, c_{x}$ und $c_{y}$ in die Formel der Ausgleichsfunktion - jeweils für Breiten- und Fahrtrichtung - eingesetzt, so können die Funktionsverläufe prognostiziert werden. Die forstliche Praxis erhält mit diesem auf empirischer Grundlage beruhenden Modell erstmals die Gelegenheit, die Druckverteilungen unter einer standardisierten Sandschicht für den größten Teil der auf den Forstmaschinen montierten Reifen vorherzusagen. Der Gültigkeitsbereich erstreckt sich dabei auf Reifen, deren Parameter innerhalb der Grenzwerte des bemessenen Reifenkollektivs liegen.

Die Interpretation ab welchem Kontaktflächendruck eine Bodenstrukturveränderung eintritt bzw. von einem Bodenschaden zu sprechen ist, bleibt den Bodenkundlern vorbehalten. Die über das Prognosemodell ausgegebenen Kontaktflächendrücke liefern Anhaltspunkte, die von Bodenwissenschaftlern aufgegriffen werden sollten, um entsprechende Strategien zur Risikominimierung vorzuschlagen und so den Zielerreichungsgrad in Bezug auf den Bodenschutz zu maximieren. Dabei stellt sich die konkrete Frage, ab welchen Kontaktflächendrücken Bodenstrukturveränderungen in Grenzen zu halten sind, so dass sie bodenökologisch und biologisch gar nicht relevant werden oder dass sich die Bodenstruktur in überschaubaren Zeiträumen wieder regeneriert.

Aufgrund der komplizierten Wechselwirkungen in den Waldböden konnten Gefährdungsschwellenwerte trotz intensiver Forschung bisher nicht hinreichend formuliert bzw. abgesichert werden.

Für den landwirtschaftlichen Bereich ermittelte WEIBBACH (2001b) mit Hilfe von Schlauchsonden maximale Bodendrücke und setzte diese ins Verhältnis mit Bodenparametern. Aus diesen Ergebnissen leitete er zwei Schwellenwerte für eine tolerierbare Bodenbelastung (Ober- und Unterboden) ab. Auf lockerem Boden wird ein Kontaktflächendruck von weniger als 1,0 bar empfohlen. Abgesetzte Böden zum Zeitpunkt der Ernte könnten deutlich höhere Drücke aufnehmen, allerdings sollte ein Bodendruck von 2,0 bar nicht überschritten werden. Darüber hinaus führt WEIBBACH (2001a) an, dass bis zu einem Kontaktflächendruck von 1,5 bar keine Beeinträchtigungen für das Pflanzenwachstum zu erwarten sind. Erst nach überschreiten des Druckes von 2,0 bar nimmt die Wurzelzahl signifikant ab. Der aufgebrachte Druck wird in Abhängigkeit der Lastabstützungsfläche, des Bodentyps, der Bodenfeuchte und des Bearbeitungszustandes verschieden stark über die Tiefe abgebaut.

Für das Befahren von Waldböden nennen MATTHIES ET AL. (1995) einen statischen Bodendruck von 0,5 bar. Wird dieser Schwellenwert beim Befahren nicht überschritten, so bleibt die natürliche Bodenstruktur weitgehend erhalten. Bei einer Überschreitung dieses Wertes seien ausnahmslos starke und tiefreichende Bodenstrukturveränderungen bis in Tiefen von maximal 
$35 \mathrm{~cm}$ zu erwarten. Kernpunkte seiner Überlegungen waren die Festlegung der Grenzwassergehalte, die ein bodenstrukturverträgliches Befahren bei bestimmten statischen Bodendrücken auf bestimmten Standorten zulassen. Hierzu untersuchten MATTHIES ET AL. (1995) auf 12 Standorten die Befahrungsauswirkungen auf die Bodenstruktur von Waldböden. Unter praxisnahen Einsatzbedingungen kamen insgesamt 13 Versuchsfahrzeuge (Harvester, Forwarder, Forstspezialschlepper und Landwirtschaftliche Schlepper mit Forstausrüstung) zum Einsatz. Die Einsatzmassen dieser Maschinen lagen zwischen 3,5 $\mathrm{t}$ und $17 \mathrm{t}$ bei mittleren Kontaktflächendrücken von ca. 0,35 bar bis 0,8 bar. Zur Berechnung dieses statischen Kontaktflächendruckes für die einzelnen Maschinen benutzen MATTHIES ET AL. (1995) die sogenannte „SkogForsk-Formel":

$$
\begin{aligned}
& \mathrm{p}=\mathrm{F} / \mathrm{B}{ }^{*} \mathrm{r} \\
& \mathrm{p}=\text { Kontaktflächendruck }\left[\mathrm{kPa}^{30}\right] \\
& \mathrm{F}=\text { Radlast }[\mathrm{kN}] \\
& \mathrm{B}=\text { Reifenbreite }[\mathrm{m}] \\
& \mathrm{r}=\text { Radradius }[\mathrm{m}]
\end{aligned}
$$

Die errechneten mittleren Kontaktflächendrücke wurden mit entsprechenden Bodenstrukturveränderungen verglichen. Dabei kamen MATTHIES ET AL. (1995) zu dem Ergebnis, dass ein Schwellenwert von 0,5 bar als tolerierbare Bodenbelastung gilt. In der SkogForsk-Formel finden der Reifenfülldruck, die Tragfähigkeit und weitere Reifenparameter allerdings keine Anwendung. Wie in den Untersuchungen des PrAllCon-Projekts festgestellt, darf aber der Einfluss des Fülldruckes und dessen Auswirkungen auf den Bodendruck nicht unberücksichtigt bleiben. Für eine Herleitung des Kontaktflächendruckes nach heutigem Kenntnisstand ist diese Formel deshalb nicht ausreichend. Der von MATTHIES ET AL. (1995) angegebene Schwellenwert ist demnach nicht mehr geeignet und es bedarf einer dringenden Anpassung. Wie SAARILAHTI (2002) betonte, gibt die SkogForsk-Formel die maximale Kontaktfläche aus und das führt zu einem hypothetisch minimalen Kontaktflächendruck.

Werden die Werte der über das Prognosemodell ermittelten maximalen und mittleren Drücke $\left(a_{m}\right.$ und $\left.a_{\text {mean }}\right)$ mit der SkogForsk-Formel verglichen, so sind deutlich die Auswirkungen eines variierenden Reifenfülldruckes zu erkennen (Abb. 57). Hierzu wurde exemplarisch der Reifen T23 herangezogen und in die jeweiligen Formeln eingesetzt. Radlast $(25 \mathrm{kN})$, Nennbreite $(700 \mathrm{~mm})$ und Durchmesser $(1350 \mathrm{~mm}$ ) werden dabei konstant gehalten. Um den Einfluss des Reifeninnendruckes sichtbar zu machen, variiert dieser um die im Rahmen des Projekts PrAllCon gewählten sieben Fülldruckstufen. Wie in Abbildung 57 zu sehen, wird für die SkogForskFormel immer den selben Konatkflächendruck ausgewiesen. Hingegen lassen die Werte für $a_{m}$ und $a_{m e a n}$ ein lineares Anwachsen des Kontaktflächendruckes erkennen. 


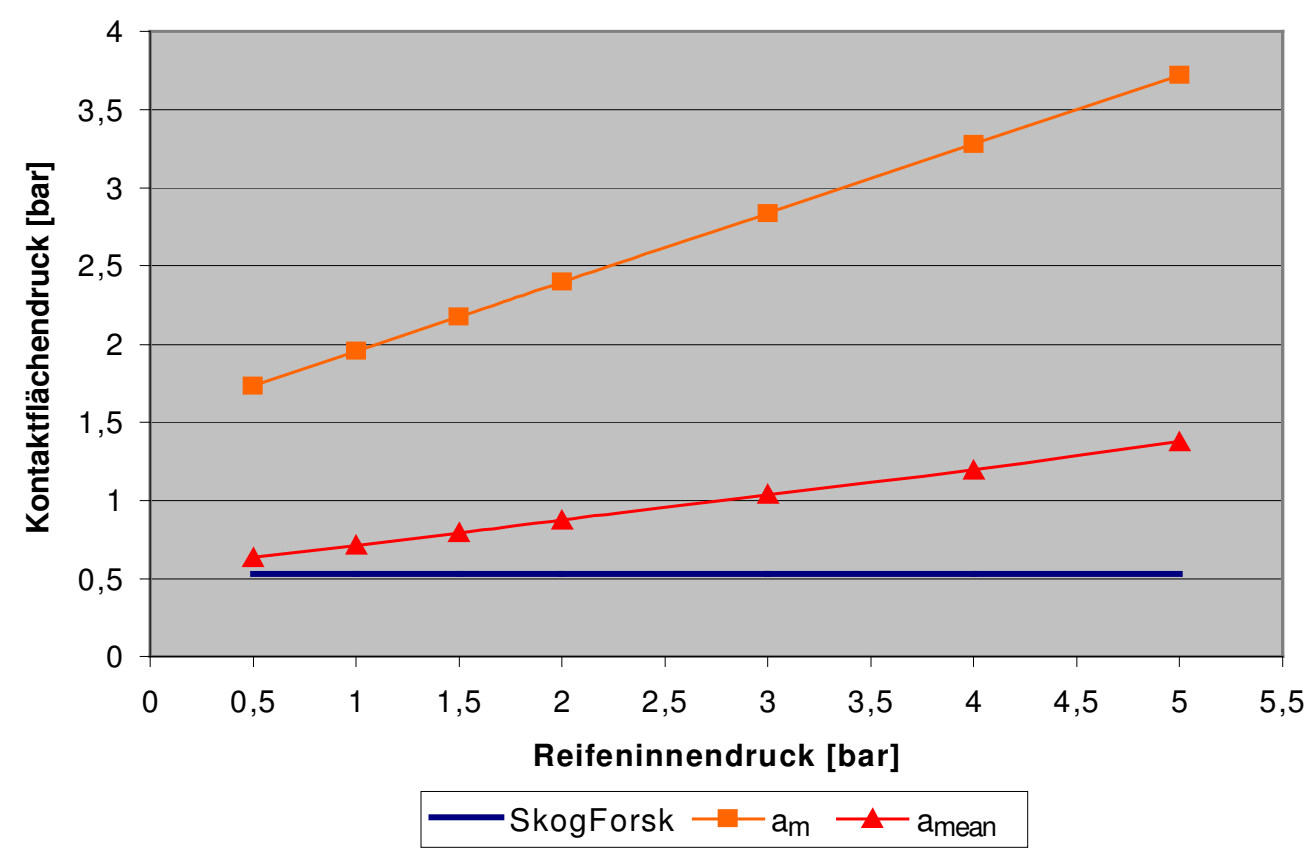

Abbildung 57: Darstellung des Kontaktflächendruckverlaufs der SkogForsk-Formel und der aus dem Prognosemodell abgeleiteten Werte $a_{m}$ und $a_{m e a n}$

In der Literatur wird dieser Schwellenwert (0,5 bar) hingegen immer wieder als ökologisches Bewertungskriterium bei Befahrungen angeführt. So stellt HAUCK (2001) fest, dass die heutigen Forstmaschinen deutlich über dem bislang in der Literatur genannten Niveau von 0,5 bar, unterhalb dessen keine negativen Veränderungen auftreten sollen, liegen. Des Weiteren führt er aus, dass für ein größeres Standortspektrum mit Schwellenwerten unter 1,0 bar gerechnet werden muss. WEHNER (2002) kommt im Rahmen von Befahrungsversuchen zu dem Ergebnis, sofern von einem Grenzwert von 0,5 bar ausgeganen wird, oberhalb dessen eine schadarme bzw. schadfreie Befahrung nicht mehr möglich ist, eine Bodenschonung in keinem Fall attestiert werden kann. Bodendrücke erreichen noch Tiefen bis $40 \mathrm{~cm}$, die den Grenzwert von 0,5 bar weit überschreiten. WEHNER (2002) verweist darauf, dass eine Regeneration befahrener Böden vor allem in Bereichen stattfindet, die durch Bodenfauna und -flora erschlossen werden können und demzufolge mit einer erschwerten Regeneration des Bodensubstrates unterhalb von $20-30 \mathrm{~cm}$ zu rechnen ist. Die daraus gezogenen Schlussfolgerungen sind zu überdenken, denn es ist zu vermuten, dass eine Neuberechung mit einer Verschiebung dieses Schwellenwertes einhergeht.

Auch für die vom Kuratorium für Waldarbeit und Forsttechnik (KWF) durchgeführten FPAPrüfung findet die SkogForsk-Formel Anwendung (GERDSEN UND GRAUPNER, 1998). In der Prüfgrundlage zur FPA-Prüfung für Tragschlepper (KURATORIUM FÜR WALDARBEIT UND FORSTTECHNIK, 1999) wird hierzu festgestellt, dass bis zum Vorliegen besserer Bewertungskriterien der Kontaktflächendruck von Rädern auf weichem Boden näherungsweise nach einer empirischen Beziehung von SkogForsk errechnet wird. Im Rahmen der FPA-Prüfung wurden z. B. für Tragschlepper und Harvester Kontaktflächendrücke zwischen 0,6 bar und 1,3 bar ermittelt. Diese Werte sind ebenfalls aufgrund der Nichtberücksichtigung des Reifenfülldruckes kritisch zu betrachten. Um die Bodenbelastung bei Befahrung zu beschränken, entwickelte das KWF u. a. ein "Bewertungsschema zur Bodenbelastung von Großmaschinen im Forst" (WEISE, 
2002). Mit diesem Schema können verschiedene selbstfahrende Forstarbeitsmaschinen in ihrer Wirkung auf den Boden unter Angabe von Reifeninnendruck, Radlast, Antriebskonzept, Reifendimensionen und Wasserfüllung miteinander verglichen werden. Zur Bewertung wurde eine gewichtete Punktebewertung als Beurteilungsverfahren gewählt. Dieses Schema bedient sich dabei ausschließlich messbarer Größen am Fahrzeug, ohne den Wirkmechanismus dieser Größen auf den Boden zu kennen. Die hieraus abgeleiteten Empfehlungen sind dementsprechend nur unter Vorbehalt zu betrachten.

Folglich sind die nummerisch abgeleiteten Kontaktflächendrücke auf Grundlage der „SkogForsk-Formel" nicht mit dem tatsächlich unter einem Reifen auftretenden Bodendruck vergleichbar, da der Reifen den Kontaktflächendruck in seiner Aufstandsfläche nicht gleichmäßig auf den Boden überträgt. Als kurzfristig zu formulierendes Ziel sollten deshalb von den Bodenwissenschaftlern abgesicherte Grenzwerte entwickelt werden. Die PrAllCon-Ergebnisse stellen eine physikalisch orientierte Grundlage dar, an der sich vom technischen Aspekt ausgerichtet werden kann.

Abschließend wird der über das entwickelte Prognosemodell errechnete Maximalwert $a_{m}$ mit den errechneten Drücken einiger etablierter Modelle verglichen. An dieser Stelle sei nochmals erwähnt, dass $a_{m}$ den Betrag des Maximums der Glockenkurvenfunktion (in unserem Modell also den jeweiligen Spitzendruck) kennzeichnet. Für den Vergleich werden die von SAARILAHTI (2002) zusammengefassten Flächen- und Druckermittlungsverfahren übernommen und auf vier ausgewählte Reifen angewendet. Bei diesen Reifen handelt es sich um zwei Exemplare (T23, N30), die vorwiegend auf Harvestern und Forwardern montiert werden, jeweils ein Exemplar (N07), das auf Landwirtschaftlichen Schleppern mit Forstausrüstung und (T12) auf Schleppern zum Einsatz kommt. SAARILAHTI (2002) verwendete in seiner Veröffentlichung für eine Gegenüberstellung etablierter Modelle 23 Formeln zur Berechung des Kontaktflächendruckes (Nominal Ground Pressure, NGP) und acht Formeln zur Berechnung des durchschnittlichen Maximaldruckes (Mean Maximum Pressure, MMP).

Anhand der im Rahmen dieses Projekts ermittelten Reifenparameter war es nur möglich, 19 der 23 NGP Formeln zu berechnen (Tab. 23). Für vier Formeln wurden Angaben zur Spurtiefe vorausgesetzt, die nicht zur Verfügung standen. Zur Berechnung der 19 Formeln wurden für die vier Referenzreifen neben definierten Radlast- und Fülldruckstufen, die Reifenparameter Nennbreite, Durchmesser und Reifenhöhe sowie die Einfederung berücksichtigt (Anhang 5, Tab. 1.1). Da die acht ausgewählten Formeln der Maximaldruckmodelle detaillierte Maschineninformationen benötigen, wurden sie ebenfalls vom Vergleich ausgeschlossen. 
Tabelle 22: Auflistung der für einen Vergleich verwendeten 19 Druckermittlungsverfahren nach SAARILAHTI (2002) - ergänzt um die aus dem Prognosemodell abgeleiteten Gleichungen des maximalen $\left(a_{m}\right)$ und mittleren Druckes ( $\left.a_{\text {mean }}\right)$

\begin{tabular}{|r|c|l|c|l|l|}
\hline \multicolumn{5}{|c|}{ Kontaktflächendruckmodelle } \\
\hline Nr. & Formel $^{31}$ & Referenz & Nr. & Formel & Referenz \\
\hline 1 & 3.1 .1 & Swedish Formula (SkogForsk) & 12 & 3.8 .5 & GoDBOLE ET AL. (1993) \\
\hline 2 & 3.2 .4 & SCHWANGHART (1990) & 13 & 3.8 .6 & GoDBOLE ET AL. (1993) \\
\hline 3 & 3.3 .1 & KOMANDI (1990) & 14 & 3.9 .1 & DWYER (1984) \\
\hline 4 & 3.4 .1 & SILVERSIDES UND SUNDBERG (1989) & 15 & 3.11 .3 & FEBO UND PESSINA (1987) \\
\hline 5 & 3.5 .2 & GRECENKO (1995) & 16 & 3.12 .2 & STEINER (1979) \\
\hline 6 & 3.5 .3 & GRECENKO (1995) & 17 & 3.15 .1 & KOOLEN ET AL. (1992) \\
\hline 7 & 3.6 .1 & KRICK (1969) & 18 & 3.16 .1 & MATTHIES UND ZIESAK (2001) \\
\hline 8 & 3.6 .3 & KRICK (1969) & 19 & 3.17 .1 & BOLLING (1985) \\
\hline 9 & 3.6 .4 & KRICK (1969) & 20 & - & am \\
\hline 10 & 3.7 .4 & LYASKO (1994) & 21 & - & amean \\
\hline 11 & 3.7 .9 & LYASKO (1994) & - & - & \\
\hline
\end{tabular}

In Abbildung 58 sind die berechneten Werte der verwendeten 19 Formeln aus 14 Modellen denen über das Prognosemodell ermittelten Maximalwerten $\left(\mathrm{a}_{\mathrm{m}}\right)$ und den daraus berechneten Durchschnittswerten ( $a_{\text {mean }}$ ) gegenübergestellt. Die Druckwerte der vier Referenzreifen wurden bei einem Fülldruck von 2,5 bar und einer Radlast von 25 kN ermittelt.

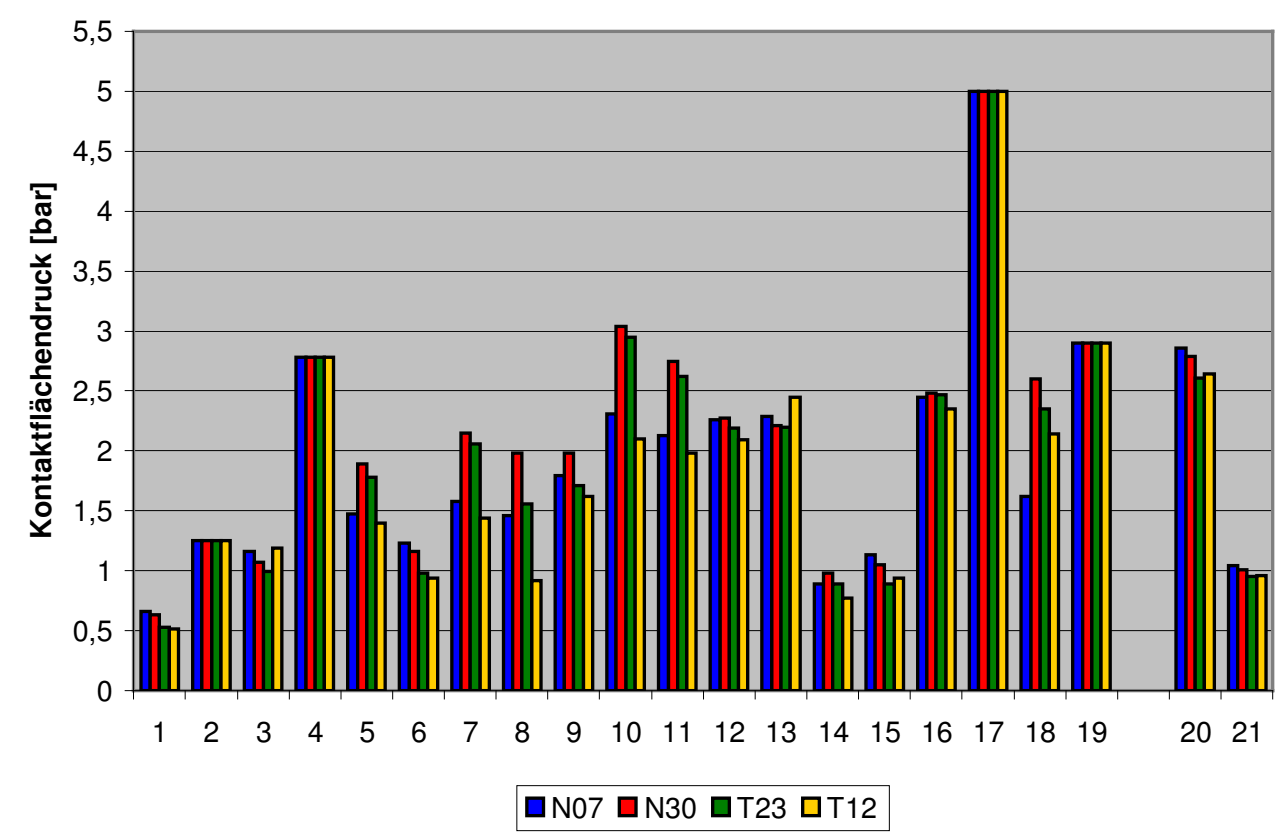

Abbildung 58: Darstellung der Druckwerte der vier Referenzreifen für die verwendeten Formeln

${ }^{31}$ Verzeichnis der zur Berechung der Kontaktflächendrücke verwendeten Formeln nach SAARILAHTI (2002). 
Ein Vergleich der Kontaktflächendrücke bringt zunächst nur wenig Aufschluss über die Güte der aufgestellten Berechnungswege. Es fällt auf, dass die Resultate hohe Abweichungen aufweisen. Die Erkenntnis, mit den eigenen über das Prognosemodell gewonnenen Maximalwerten $\left(a_{m}\right)$ im oberen Bereich zu liegen ist ohne Belang, denn viele der Vergleichsmodelle geben durchschnittliche Kontaktflächendrücke aus, so dass zur besseren Orientierung die mitaufge-

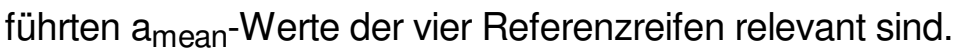

Im Bereich der $\mathrm{a}_{m}$-Werte (2,5 - 3,0 bar) befinden sich die ermittelten Kontaktflächendrücke der Modelle von SILVERSIDES (1989), BoLLING (1985) sowie LYASKO (1994), GODBOLE ET AL. (1993) und STEINER (1979). Dabei fällt auf, dass SILVERSIDES (1989) und BOLLING (1985) nur Radlast und Fülldruck als Parameter in ihre Berechnungen einbeziehen und somit für alle vier Reifen gleiche Kontaktflächendrücke ausweisen. Noch entscheidender ist, dass die fünf genannten Modelle mittlere Kontaktflächendrücke ausgeben, die im Bereich der über das Prognosemodell ermittelten Maximaldrücke liegen. Werden über den Faktor 2,74 zur besseren Orientierung die gemittelten Drücke $a_{\text {mean }}$ (ca. 1,0 bar) berechnet, so kommt man in den Bereich der Kontaktflächendrücke der Modelle von DWYER (1984), FEBO UND PESSINA (1987) und KOMANDI (1990). Bei genauerer Betrachtung dieser drei Modelle ist zu erkennen, dass alle Formeln Angaben über Radlast, Nennbreite und Durchmesser benötigen. KomANDI (1990) betrachtet zusätzlich den Fülldruck und dementsprechend alle Parameter, die auch im Prognosemodell vorhanden sind. DWYER (1984), FEBO UND PESSINA (1987) verwenden anstatt des Fülldruckes die Einfederung als relevanten Parameter. Als letzter Punkt ist innerhalb dieses Vergleichs nochmals auf die „SkogForsk-Formel“ einzugehen. Die von SAARILAHTI (2002) als Standardmodell angeführte Formel gibt die niedrigsten Kontaktflächendrücke (ca. 0,6 bar) aus.

Dieser abschließende Vergleich der Formeln untereinander verdeutlicht nochmals die Heterogenität in Bezug auf die Voraussage von Kontaktflächendrücken unter Forst- und Landwirtschaftsreifen. Eine Einordnung des hier vorgestellten Prognosemodells bezüglich der Aussagekraft und der Wertigkeit für bodenökologische Interpretationen muss sich allerdings erst etablieren. Die Ergebnisse des PrAllCon-Projekts können allerdings dazu beitragen, das Risiko von Bodenstrukturveränderungen besser einzuschätzen und eine gezielte Auswahl von Reifen und Maschinen einleiten. Gerade für die technische Ausstattung der Forstmaschinen geben die Untersuchungen zur Bereifung und der Druckverteilung konkrete Hinweise. Zudem werden der Industrie für die Produktentwicklung von forstwirtschaftlichen Reifen (und Maschinen) weitere Anhaltspunkte zur Verfügung gestellt. 


\section{Zusammenfassung}

Technische und ökologische Wirkungen selbstfahrender Forstmaschinen werden unter anderem beeinflusst von der Verteilung des Druckes auf der Kontaktfläche unter dem Rad. Gerade unter typisch forstlichen Rahmenbedingungen ist diese Verteilung noch nicht erforscht. Das Forschungsprojekt Druckverteilung auf Kontaktflächen unter Forstreifen oder Pressure Allocation on Contact Areas under Forest Tires (PrAllCon) hatte zum Ziel, diese Kenntnislücke zu schließen.

Eine Marktanalyse brachte Aufschluss über eine detaillierte Zusammensetzung der Bereifung selbstfahrender Forstmaschinen in Deutschlands Wäldern. Parallel dazu erfolgte die Entwicklung einer geeigneten Messtechnik, die bei der Erfassung der Kontaktflächendruckverteilung auch den Stützanteil zwischen den Stollen der Reifenprofilierung berücksichtigt (Messung mit definierten Sand). Zur Präparierung der Neureifen auf die Messungen mussten diese „eingefahren"werden (Walkmaschine). Eine hydraulische Radlastsimulation mit analog messbarer Verteilung des Kontaktflächendruckes und dessen digitaler Visualisierung lieferte die gewünschten Daten.

Generell konnte festgestellt werden, dass sich mit zunehmender Radlast und steigendem Fülldruck der Schwerpunkt der Druckbelastung in der Mitte der Kontaktfläche konzentriert und stetig zur Peripherie hin abnimmt. Die weitere Verarbeitung und Analyse dieser in gesteuerter Abhängigkeit von Fülldruck und Radlast erhobenen Verteilungen des Druckes (Messdaten) erfolgte mit am IFA entwickelten Programmen in Kombination mit statistischer Analysesoftware. Die Auswertungen zeigten, dass der Druck sich durchweg in Form einer Glocke mit elliptischer Querschnittsfläche verteilt. Die maximalen Druckwerte wurden mittels in Breiten- und Fahrtrichtung vorgenommener Vertikalschnitte durch die Verteilungsglocken ermittelt. Diese glockenförmigen Druckverläufe konnten mit einer Ausgleichsfunktion erfasst werden. Bestimmtheitsmaße von durchschnittlich $92 \%$ erlaubten jede gemessene Kontaktflächendruckverteilung zu beschreiben und gültige Vorhersagen für das gesamte Reifenkollektiv zu entwickeln. Regressionsanalysen gaben Aufschluss darüber, dass die Druckverteilung neben Radlast und Fülldruck durch die Reifenparameter Nennbreite und Durchmesser beeinflusst wird. Die Entwicklung eines Prognosemodells erlaubt zudem die Druckverteilungen in Abhängigkeit dieser Variablen in Breiten- und Fahrtrichtungsausprägung vorherzusagen. Der Gültigkeitsbereich erstreckt sich dabei auf Reifen, deren Parameter innerhalb der Grenzwerte des bemessenen Kollektivs liegen.

Neben den maximalen Drücken wurden auch die mittleren Drücke untersucht. Zwischen den Maximaldrücken und den Maxima dieser gemittelten Druckverteilungen bestand ein plausible Korrelation, die sich mit einer Einfachregression herleiten ließ. Es wurde ein Faktor von 2,74 abgeleitet, mit dem bei Kenntnis des maximalen Druckes auf den mittleren Druck geschlossen werden kann. 


\section{Summary}

Technical and ecological effects of automated forestry machines is influenced in part by the distribution of pressure on the surface beneath the wheel. However, it is precisely under typical forest conditions that these effects have not yet been examined. This research project Druckverteilung auf Kontaktflächen unter Forstreifen or Pressure Allocation on Contact Areas under Forest Tires (PrAlICon) was designed to supply this missing information.

A market analysis provided a detailed report of the composition of the tires found on automated machines used in Germany's forests. Parallel to this, a technique was developed by which the pressure allocation on the contact surface could be measured while also taking into account the degree of support between the lugs of the tire profile (measured with sand of defined composition). In order to prepare the new tires for this test they had to be "driven" several times using a rolling machine. The desired data were then obtained by means of a hydraulic simulation of wheel load with analog calculation of surface area pressure distribution and it's subsequent digital visualisation.

In general it could be ascertained that the focus of the pressure load was concentrated in the middle of the contact area and decreased steadily towards the periphery. This became more pronounced as wheel load and tire pressure increased. Further processing and analysis of these pressure distributions as a function of wheel load and tire pressure was done by using programs developed by the IFA together with software for statistical analysis. The results demonstrated that the pressure did indeed have a bell-shaped distribution with an elliptical crosssection. The maximum pressure values were calculated using width and length measurements from vertical cross sections of the respective distribution curves. These bell-shaped pressure progressions could be represented in a function of determination. An average coefficient of determination of $92 \%$ enabled the determination of every measured contact area pressure distribution as well as the prediction of pressure distribution for the entire tire collective. Analysis of regression curves showed that not only wheel load and tire pressure but also the distribution of pressure over the contact area are influenced by the tire parameters: width and diameter. The creation of a prognosis model makes it also possible to predict the pressure distribution as dependent on the variables tire width and length. The validity of this method extends only to tires whose parameters lay within those of the collective measured in this study.

In addition to maximum pressures mean pressures were also examined. Between the maximum pressures and the maxima of the mean pressure distributions there existed a plausible correlation that could be derived using simple regression. The factor was calculated to be 2.74. Thus, given a maximum pressure, the corresponding mean pressure can be inferred. 


\section{Literaturverzeichnis}

Zeitschriftenabkürzungen:

AFZ-Der Wald:

AFJZ:

FoHo:

$F \& T$ :

FTI:

FwCbl:

HZBL:

KTBL-Schrift:
Allgemeine Forst Zeitschrift

Allgemeine Forst- und Jagdzeitung

Forst und Holz

Forst \& Technik

Forsttechnische Informationen

Forstwissenschaftliches Centralblatt

Holz- Zentralblatt

Kuratorium für Technik und Bauwesen in der Landwirtschaft

ABEELS, P. F. J. (1983): Überlegungen zur Herstellung von Reifen für forstwisschenschaftliche Fahrzeuge. FwCbl., Nr. 102, S. 80-86.

ARNOLD, K.-D. (2002): Wohin wird sich die forstliche Technik in den nächsten Jahren entwickeln? FoHo, Nr. 12, S. 363-368.

BACKFISCH, K. P. UND HEINZ, D. S. (2000): Das neue Reifenbuch. Motorbuch Verlag, Stuttgart, S. 287.

BACKHAUS, G. (1992): Reifendruckregelsystem als Forstausrüstung. AFZ-Der Wald, Nr. 6, S. 6.

BACKHAUS, G. (1993): Reifendruckverstellanlagen und ihr Nutzen beim Forstmaschineneinsatz. AFZ-Der Wald, Nr. 15, S.761-763.

BACKHAUS, G. (1994): Reifendruck-Verstellanlagen. Konsequenzen für die Bereifung von Forstmaschinen bei verstellbarem Reifenluftdruck. F \& T, Nr. 12, S. 12-17.

BACKHAUS, G. (1998): Richtiger Luftdruck im Forstreifen. F \& T, Nr. 3, S. 6-9.

BACKHAUS, K.; ERICHSON, B.; PLINKE, W. UND WEIBER, R. (2000): Multivariate Analysemethoden. Eine anwendungsorientierte Einführung. 9. überarbeitete und erweiterte Auflage, Springer-Verlag, Berlin, $661 \mathrm{~S}$.

BARUM (2003): Landwirtschaftsreifen - Technischer Ratgeber 2003/2004. Hannover, 78 S..

BECKER, G.; HOFMANN, R. UND GROSS, M. (1986): Boden- und Wurzelschäden durch Befahrung von Waldbeständen. FoHo, Nr. 19, S. 367-370.

BENECKE, P. (1982): Auswirkungen des Befahrens mit schweren Fahrzeugen auf bodenphysikalische Kennwerte. Mitt. d. Dt. Bodenkunkdl. Gesellsch., Nr. 34, S. 181-185.

BERNHARD, B. UND SCHLOTTER, V. (2001): Reifendynamik bei Wasserfüllung. Landtechnik, Nr. 56, S. 14-15.

BoLLING, I. (1985): How to predict the soil compaction of agricultural tires. Anniv. Vol. of the Int. Conf. on Soil Dynamics and Commemoration of the 50th Anniv. of the Nat. Tillage Mach. Lab., Auburn, Alabama, USA (1985) H.5, S.936-952.

BoLLING, I. (1986): Beanspruchung des Bodens bei Schlepper- und Maschineneinsatz. KTBLSchrift, Nr. 308, S. 49-71.

BREDBERG, C. UND WÄSTERLUND, J. (1983): Wurzel- und Bodenschäden durch Fahrzeuge. FwCbl., Nr. 102, S. 86-98.

Bronstein, I. N; SemendjajeW, K. A.; Musiol, G. Und MüHLIG, H. (2001) Taschenbuch der Mathematik. 5. überarbeitete und erweiterte Auflage, Verlag Harri Deutsch, Frankfurt am Main, $1191 \mathrm{~S}$. 
BRosIUS, F. (1998): SPSS 8 Professionelle Statistik unter Windows. MITP-Verlag, Bonn, $1075 \mathrm{~S}$.

BUNDESBODENSCHUTZGESETZ (1998): vom 17. März, Bundesgesetzblatt, S. 502.

BUNDESNATURSCHUTZGESETZ (1998): vom 21. September, Bundesgesetzblatt, S. 2994.

COMPUTER MESSTECHNIK VERTRIEB CMV HOOVEN (2001): Generalimporteur und alleiniges Vertriebsunternehmen sämtlicher Tekscan-Produkte in der Bundesrepublik Deutschland, Produktinformationen, Mönchengladbach.

CRECENKO, A. (1995): Tyre footprint area on hard ground computed from catalogue values. Journal of Terramechanics, 32 (6), S. 325-333.

DENNINGER, W. (2002): Stand der hochmechanisierten Holzernte in Niedersachsen. F \& T, Nr. 7, S. 14-16.

DEUTSCHER VERBAND FÜR WASSERWIRTSCHAFT UND KULTURBAU e. V. (1997): Gefügestabilität ackerbaulich genutzter Mineralböden - Teil II: Auflastabhängige Veränderung von bodenphysikalischen Kennwerten. Merkblatt zur Wasserwirtschaft, Nr. 235, 7 S.

DISERENS, E. (2002): Ermittlung der Reifen-Kontaktfläche im Feld mittels Rechenmodell. Eidgenössische Forschungsanstalt für Agrarwirtschaft und Landtechnik (FAT), Bericht-Nr. $582,12 \mathrm{~S}$.

DisERENS, E. (2004): Wechselwirkung zwischen Fahrwerk und Ackerboden. Eidgenössische Forschungsanstalt für Agrarwirtschaft und Landtechnik (FAT), Bericht-Nr. 613, $16 \mathrm{~S}$.

DISERENS, E.; ANKEN, T.; WEISKOPF, P. UND ZIHLMANN, U. (1998): Tragen Unterböden größere Lasten als erwartet? Agrarforschung, Nr. 5 (1), S. 9-12.

DöLL, H. (1998): Bewertung von Landwirtschaftsreifen nach agrotechnischen, ökonomischen und technisch-energetischen Kriterien. FTI, Nr. 3, S. 36-38.

DürR, H. J.; PetelKAU, H. UND SOMmeR, C. (1995): Literaturstudie Bodenverdichtung. Forschungsbericht Bundesumweltamt, Nr. 55, $203 \mathrm{~S}$.

DWYER, M. J. (1984): Tractive performance of wheeled vehicles. Journal of Terramechanics, 21 (1), S. 19-34

EBEL, A. UND JACKE, H. (2003): Marktanalyse der auf selbstfahrenden Forstmaschinen montierten Reifen. AFZ-Der Wald, Nr. 10, S. 482-484.

ERLER, J. (2000): Forsttechnik: Verfahrensbewertung. Ulmer-Verlag, Stuttgart, 248 S.

ESSER ET. AL. (2000): Der Forstwirt. Herausgeber: Waldarbeitsschulen der Bundesrepublik Deutschland. 3. bearbeitete Auflage, Ulmer-Verlag, Stuttgart, $641 \mathrm{~S}$.

EuRopaEn TYRE AND RIM TECHNICAL ORgANIZATION (2002). Standard Manual 2002. Brüssel.

FEBo, P. UND PESSINA, D. (1987): Contact area tests of a new wide section agricultural tyre. Proc. of the 9th Int. Conf. of the ISTVS, Barcelona, Spain, 31.-04.09.1987, S. 236-243.

FENSEL, C. (2002): Mündliche Mitteilung. Bavaria Spezial Rad GmbH (BSR), Geschäftsführender Gesellschafter.

FENSEL, C. (2003): Mündliche Mitteilung. Bavaria Spezial Rad GmbH (BSR), Geschäftsführender Gesellschafter.

ForBRIG, A. (2000): Konzeption und Anwendung eines Informationssystems über Forstmaschinen auf der Grundlage von Maschinenbuchführung, Leistungsnachweisen und technischen Daten. KWF-Bericht, Nr. 29, zugl. Dissertation der TU München, 213 S.

FOREST STEWARDSHIP COUNCIL (2004): Leitfaden für Waldbesitzer und Forstbetriebe - Bestimmungen der Deutschen FSC-Standards. (Stand: 21.04.2004). 
GAERTIG, T.; SCHACK-KIRCHNER, H. UND HILDEBRAND, E. E. (2001): Steuert Gasdurchlässigkeit im Boden Feinstwurzeldichte und Vitalität bei Eiche? AFZ-Der Wald, Nr. 25, S. 13441347.

GERDSEN, G. (1997): Zum Einfluss von Wasserfüllungen in Forstreifen. FTI, Nr. 9, S. 103-106.

GERDSEN, G. UND GRAUPNER, J. (1998): Der Kontaktflächendruck bei Forstmaschinen - eine wichtige ökologische Kennziffer zur Beurteilung der Bodenpfleglichkeit. FTI, Nr. 3, S. 35-36.

GIESEN, T. (2003): Der Begriff der guten forstlichen Praxis in der Forstwirtschaft. AFZ-Der Wald, Nr. 8, S. 392-395.

Godbole, R.; Alcock, R. Und Hettiaratchi, D. (1993): The prediction of tractive performance on soil surfaces. Journal of Terramechanics 30 (6), S. 443-459.

GRECENKO, A. (1995): Tyre footprint area on hard ground computed from catalogue value. Journal of Terramechanics 32 (6), S. 325-333.

GYSI, M. UND GRUNDER, F. (1999): Zusammenhang zwischen Kontaktfläche und Pneuinnendruck. Agrarforschung, Nr. 6 (1), S. 9-12.

HAMBERGER, J. (2002): GPS als Mittel zum umweltschonenden Maschineneinsatz: Navigation von Forstmaschinen und Dokumentation ihrer Fahrbewegungen. Forstliche Forschungsberichte München, Nr. 188, zugl. Dissertation der TU München, 192 S.

HAUK, B. (2001): Aspekte des Bodenschutzes bei der Entwicklung, beim Kauf und dem Einsatz von Forstmaschinen. FTI, Nr. 4, S. 41-46.

HILDEBRAND, E. E. (1981): Befahrung und Bodenverdichtung unter dem Aspekt der Bodenfunktion als Waldstandort. Mitt. d. Dt. Bodenkdl. Gesellsch., Nr. 32, S. 51-85.

HILDEBRAND, E. E. UND WIEBEL, M. (1982): Der Einfluss von Befahrung auf die Qualität von Forststandorten. AFJZ, Nr. 153, S. 24-34.

Hildebrand, E. E.; Puls, C.; GaeRtig, T. und Schack-KIRChneR, H. (2000): Flächige Bodenverformung durch Befahren. AFZ-Der Wald, Nr. 13, S. 683-686.

HOFMANN, R. (1989): Bodenschäden durch Forstmaschineneinsatz. Dissertation der Universität Freiburg, $141 \mathrm{~S}$.

HOFMANN, R. UND BECKER, G. (1990): Bodenschäden im Wald durch den Einsatz von Forstmaschinen. AFZ-Der Wald, Nr. 20, S. 478-480.

ISENSEE, E. UND WEIBBACH, M. (1994): Kontaktflächendruck unter bodenschonenden Reifen. Landtechnik, Nr. 1, S. 22-24.

JACKE H. (2005a): PrAllCon: Neues über Forstreifen - Reifen als Feder-Masse-Systeme. Unveröffentlicht, $16 \mathrm{~S}$.

JACKE H. (2005b): PrAllCon: Neues über Forstreifen - Zur Druckverteilung auf hartem Untergrund. Unveröffentlicht, $16 \mathrm{~S}$.

JACKE, H. (1980): Zur Technik der Regressionsrechnung bei der Analyse forstlicher Arbeitszeitstudiendaten. Dissertation der Universität Göttingen, 204 S.

JACKE, H. (1999): Öko-Controling im Maschineneinsatz - oder: Wie man Leistung steigert, ohne der Umwelt zu schaden. FTI, Nr. 5+6, S. 41-44.

JACKE, H. UND DREWES, D. (2004): Zur Nutzungsdauer selbstfahrender Arbeitsmaschinen in der Forstwirtschaft. FoHo, Nr. 12, S. 587-592.

KoOlen, A. J. P.; LeRINK, D. A.; KuRstJens, J. J. H.; van DER AKKER \& ARTS, W. B. M. (1992): Prediction of aspects of soil-wheel systems. Soil and Tillage Research 24, S. 381-396.

KRICK, G (1969): Radial and shear stress distribution under rigid wheels and pneumatic tyres operating on yielding soils with consideration of tyre deformation. Journal of Terramechanics, 6 (3), S. 73-98. 
KRIEGER, R. (2003): Mündliche Mitteilung. CGS Reifen Deutschland GmbH / Director Research \& Development.

KRIEGER, R. (2004): Mündliche Mitteilung. CGS Reifen Deutschland GmbH / Director Research \& Development.

KURATORIUM FÜR WALDARBEIT UND FORSTTECHNIK (1999): Prüfgrundlage zur FPA-Prüfung Tragschlepper. $55 \mathrm{~S}$.

KURATORIUM FÜR WALDARBEIT UND FORSTTECHNIK (2001): FPA-Verzeichnis, Teil 1 bis 3.

KURATORIUM FÜR WALDARBEIT UND FORSTTECHNIK (2003): Prüfgrundlage zur FPA-Prüfung, Kranvollernter. $89 \mathrm{~S}$.

LEINERT, S. (1981): Forsttechnik 2000 - mehr als nur ein Wintermärchen. AFZ-Der Wald, Nr. 40, S. 1260-1264.

LÖFFLER, H. (1983): Risiken moderner forstlicher Arbeitsverfahren für Boden und Bestand. FwCbl., Nr. 102, S. 65-67.

LÖFFLER, H. (1986): Bodenschäden bei der Holzernte - Ursachen, Folgen, Vorbeugung. HZBL, Nr. 149, S. 2190-2192.

LYASKO, M. I. (1994): The determination of deflection and contact characteristics of a pneumatic tire on a rigid surface. Journal of Terramechanics 31(4), S. 239-242.

MATTHIES, D. (1994): Erfassung von Bodenschäden. AFZ-Der Wald, Nr. 13, S. 723.

MATTHIES, D. (2000): Eine Qualitätsanalyse der bundesländerspezifischen BodenschutzKonzepte zum bodenschonenden Forstmaschineneinsatz. AFJZ, Nr. 3, S. 41-46.

MATTHIES, D. (2002): Zusammenstellung wichtiger Kenngrößen von Forstmaschinen nach FPA. Unveröffentlicht, $3 \mathrm{~S}$.

MATTHIES, D. UND KREMER, J. (1999): Bodenmechanische und bodenphysikalische Ergebnisse im Rahmen des Informationssystems zum bodenverträglichen Forstmaschineneinsatz. FTI, Nr. 5+6, S. 34-39.

MATTHIES, D. UND ZIESAK, M. (2001): Untersuchungen zur last- und innendruckabhängigen Aufstandsfläche von Forstspezialreifen. FTI, Nr. 9+10, S. 104-110.

MATTHIES, D.; WEIXLER, H. UND HESS, U. (1995): Befahrungsbedingte Strukturveränderungen von Waldböden. AFZ-Der Wald, Nr. 22, S. 1218-1221.

MIKKONEN, E. UND WUOLIJOKI, E. (1975): Pikatestausten suoritustekniikka. The technique of short term testing. Metsätehon katsaus 9. 5 S. Zitiert nach: SAARILAHTI, M. (2002): Modeling of the wheel and tyre. 1. Tyre an soil contact - Survey on tyre contact area and ground pressure models for studying the mobility of forest tractors. In: Haarla, R., Salo, J. (eds.): Development of a protocol for ecoefficient wood harvesting on sensitive sites (ECOWOOD), Helsinki, $43 \mathrm{~S}$.

MÜLLER, P. (2002): Mündliche Mitteilung. Trelleborg Wheel Systems / Produkt Manager Trelleborg Forst \& Farm.

MüLLER, P. (2003): Mündliche Mitteilung. Trelleborg Wheel Systems / Produkt Manager Trelleborg Forst \& Farm.

NiCK, L. UND FORBRIG, A. (2002): Forstmaschinen in Deutschland. F \& T, Nr. 7, S. 20-21.

OhrneR, G.; Matthies, D.; Kremer, J.; Kaiserauer, J; Wolf, B.; Uhl, E. Und BLAschKe, M. (2003): Vergleichende Studie zur Auswirkung von Rad- und Raupenfahrwerken auf das Wurzelsystem (Kuratoriumsprojekt A 34). Bayrische Landesanstalt für Wald- und Forstwirtschaft, $138 \mathrm{~S}$.

PотSCH, M. (1996): Messungen der Luftpermeabilität zur flächenhaften Erfassung von Verdichtungserscheinungen - Eignung und Fallbeispiel. Diplomarbeit der Universität Bayreuth, $98 \mathrm{~S}$. 
Programme for the Endorsement of Forest Certification Schemes (2005): PeFCStandards für Deutschland - Leitlinie für nachhaltige Waldbewirtschaftung zur Einbindung des Waldbesitzers in den regionalen Rahmen (Stand: 19.01.2005).

PüTZ, M. (2001): Reifenregler in der Praxis. Landwirtschaft ohne Pflug, Nr. 4, S. 19-21.

REINHARDT, D. W. (1995): Niederquerschnitt - Niederdruck-Breitreifen. AFZ-Der Wald, Nr. 9, S. 461-462.

RENIUS, K. T. (1985): Traktoren - Technik und ihre Anwendung. BLV Verlagsgesellschaft, München, $191 \mathrm{~S}$.

ROGALL, K. (1968): Die Bringungsmöglichkeiten von Langholz durch den Ackerschlepper. FTI, Nr. 9, S. 68-76.

ROWLAND, D. (1972): Tracked vehicle ground pressure and its effect on soft ground performance. Proc. of the 4th Int. Conf. of ISTVS, 24.-28.08. 1972, Stockholm, Schweden, S. 353-384.

SAARILAHTI, M. (2002): Modeling of the wheel and tyre. 1. Tyre an soil contact - Survey on tyre contact area and ground pressure models for studying the mobility of forest tractors. In: Haarla, R., Salo, J. (eds.): Development of a protocol for ecoefficient wood harvesting on sensitive sites (ECOWOOD), Helsinki, $43 \mathrm{~S}$.

SCHACK-KIRCHNER, H. UND HILDEBRAND, E. E. (1993): Struktur und Gashaushalt von Waldböden. DFG-Schlussbericht Hi 275/2-1, $186 \mathrm{~S}$.

SCHLAGHECK, H. (2000): Der deutsche Wald im 21. Jahrhundert Holzacker? Ökomuseum? Freizeitpark? AFZ-Der Wald, Nr. 16, S. 862-865.

SCHLOTTER, V. UND KUTZBACH, H. D. (2001): Innenkontur eines Traktorreifens auf festem und nachgiebigen Boden. Agrartechnische Forschung Nr. 7, S. 23-27.

SCHULZ, H. (1994): Reifen für Forstfahrzeuge - Die Reifenauswahl will sorgfältig überlegt sein. F \& T, Nr. 4, S. 22-23.

ScHULZ, H. (1995): Reifenverschleiß bei Straßenfahrt. F \& T, Nr. 10, S. 25.

SCHWANGHART, H. (1990): Measurement of contact area, contact pressure and compaction under tires in soft soil. Proc. of the 10th Int. Conf. of the ISTVS, 20.-24.08.1990, Kobe, Japan H.Vol.1, S.193-204

SCHWANGHART, H. (1993): Auswirkungen einer Luftdruckverminderung bei Landwirtschaftlichen Reifen in lockeren Boden. KTBL-Schrift, Nr. 362, S. 108-123.

SCHWIEGER, H. (1996): Untersuchung neuartiger Laufwerke und lasergestützte Erfassung der Reifen- / Bodenverformung. Dissertation der Universität Kiel, 166 S.

SEMmEL, H. UND HORN, R. (1993): Möglichkeiten zur Bestimmung der mechanischen Belastbarkeit und der Druckfortpflanzung im Boden im Hinblick auf die Ableitung von bodentyp- und maschinenspezifischen Grenzwerten. KTBL-Schrift, Nr. 362, S. 61-92.

SILVERSIDES, C. R. UND SUNDBERG, U. (1989): Operational efficiency in forestry. Vol 2. Practice. Kluver Academic Publishers, Dortrecht/Boston/London, $169 \mathrm{~S}$.

STATISTISCHES JAHRBUCH (2004): Für die Bundesrepublik Deutschland. Statistisches Bundesamt, Wiesbaden, S. 374.

STEINER, M. (1979): Analyse, Synthese und Berechnungsmethoden der TriebkraftSchlupf-Kurve von Luftreifen auf nachgiebigen Boden. Forschungsbericht Agrartechnik des Arbeitskreises Forschung und Lehre der Max-Eyth-Gesellschaft (MEG) H. 33. Dissertation der Universität München, $190 \mathrm{~S}$.

STRABENVERKEHRSGESETZ (2005): vom 14. August, Bundesgesetzblatt, S. 2412.

STRABENVERKEHRS-ZULASSUNGS-ORDNUNG (2005): vom 27. Juni, Bundesgesetzblatt, S. 1882.

STREEß, W. (2003): Hydraulik-Schaltplan (technische Zeichnung). Ehemaliger Werkstattleiter des IFA. 
SUDA, M. UND BECK, R. (2001) Anforderungen an eine universitäre forstliche Ausbildung in der Zukunft. AFZ-Der Wald, Nr. 6, S. 296-298.

TEKSCAN (2001a): Industrial Sensor Catalog. Includes: Sensor Dimensions an Pressure Ranges. Bosten.

TEKSCAN (2001b): I-Scan Pressure Measurement System - User's Manual. Bosten.

TRELLEBORG (2003): Reifenhandbuch - Niederdruckreifen TWIN für Land- und Forstwirtschaft, Industrie und Grünflächen, 2003-04. TRELLEBORG Wheel Systems. 69 S.

TÜRCKHEIM. H. E. v. (1983): Praxiserfahrungen beim Einsatz von Niederquerschnitt-Breitreifen. AFZ-Der Wald, Nr. 38, S. 1130.

VOLZ, K.-R. UND WINKEL, G. (2003): Naturschutzfachliche Kriterien zur Bewertung der guten fachlichen Praxis der Forstwirtschaft. Abschlussbericht, erstellt im Auftrag des Bundesamtes für Naturschutz am Institut für Forstpolitik, Universität Freiburg, 219 S.

WEHNER, T. (2002): Technikkonzepte und ihre Auswirkungen auf den Boden. Schriftenreihe Freiburger Forstliche Forschung. Band 18, $11 \mathrm{~S}$.

WEISE, G. (2002): Bewertungsschema zur Bodenbelastung von Großmaschinen im Forst. FTI, Nr. $1+2$, S. 10-12.

WEIBBACH, M. (2001a): Niedriger Luftdruck ist entscheidend. Landwirtschaft ohne Pflug, Nr. 4, S. $12-18$.

WEIBBACH, M. (2001b): Neue Reifenkonzepte zur Bodenschonung. Landtechnik, Nr. 2, S. $72-$ 73.

WILPERT, K. v. (1998): Möglichkeiten und Grenzen für die Definition einer ökologisch verträglichen Befahrbarkeit. FTI, Nr. 3, S. 29-34.

ZANDER, J. (1988): Experimentelle Untersuchungen über den Einfluss des Befahrens auf die Oberbodenverdichtung. FwCbl., Nr. 107, S. 112-122.

ZIESAK, M. (2004): Entwicklung eines Informationssystems zum bodenschonenden Forstmaschineneinsatz. Dissertation der TU München, $145 \mathrm{~S}$. 


\section{Anhang}

Anhang 1: Ergänzungen zum Aufbau von Rädern

Anhang 2: Ergebnisse Marktanalyse - Diagonal-/Radialreifen

Anhang 3: Übersicht der bemessenen Reifen

Anhang 4: Kontaktflächenabdrucke auf weichem Untergrund und Fotografien des Reifens Trelleborg 600/55-26.5 428

Anhang 5: Referenzreifen - Berechnungsgrößen für den Formelvergleich

Anhang 6: Kontaktflächendruckverteilungen auf weichem Untergrund (PDF-Datei auf einer CD - Dateiname „Reifenkatalog") 


\section{Anhang 1}

Tabelle 1.1: Gängige Anschlussmaße für Forsträder

\begin{tabular}{|c|c|c|c|}
\hline Lochzahl für Bolzen & $\begin{array}{c}\text { Bolzloch- } \\
\text { durchmesser } \\
{[\mathrm{mm}]}\end{array}$ & $\begin{array}{c}\text { Mittenloch- } \\
\text { durchmesser } \\
{[\mathbf{m m}]}\end{array}$ & $\begin{array}{c}\text { Lochkreis- } \\
\text { durchmesser } \\
{[\mathbf{m m}]}\end{array}$ \\
\hline 8 & $22 / 27$ & 221 & 275 \\
\hline 10 & $22 / 27$ & 281 & 335 \\
\hline
\end{tabular}

Abbildung 1.1: Tabelle Last-Index (verändert nach E.T.R.T.O., 2002)

\begin{tabular}{|c|c|c|c|c|c|c|c|c|c|c|c|c|c|}
\hline LI & $\mathrm{kg}$ & LI & $\mathrm{kg}$ & LI & $\mathrm{kg}$ & LI & $\mathrm{kg}$ & LI & $\mathrm{kg}$ & LI & $\mathrm{kg}$ & LI & $\mathrm{kg}$ \\
\hline 0 & 45 & 40 & 140 & 80 & 450 & 120 & 1400 & 160 & 4500 & 200 & 14000 & 240 & 45000 \\
\hline 1 & 46.2 & 41 & 145 & 81 & 462 & 121 & 1450 & 161 & 4625 & 201 & 14500 & 241 & 46250 \\
\hline 2 & 47.5 & 42 & 150 & 82 & 475 & 122 & 1500 & 162 & 4750 & 202 & 15000 & 242 & 47500 \\
\hline 3 & 48.7 & 43 & 155 & 83 & 487 & 123 & 1550 & 163 & 4875 & 203 & 15500 & 243 & 48750 \\
\hline 4 & 50 & 44 & 160 & 84 & 500 & 124 & 1600 & 164 & 5000 & 204 & 16000 & 244 & 50000 \\
\hline 5 & 51.5 & 45 & 165 & 85 & 515 & 125 & 1650 & 165 & 5150 & 205 & 16500 & 245 & 51500 \\
\hline 6 & 53 & 46 & 170 & 86 & 530 & 126 & 1700 & 166 & 5300 & 206 & 17000 & 246 & 53000 \\
\hline 7 & 54.5 & 47 & 175 & 87 & 545 & 127 & 1750 & 167 & 5450 & 207 & 17500 & 247 & 54500 \\
\hline 8 & 56 & 48 & 180 & 88 & 560 & 128 & 1800 & 168 & 5600 & 208 & 18000 & 248 & 56000 \\
\hline 9 & 58 & 49 & 185 & 89 & 580 & 129 & 1850 & 169 & 5800 & 209 & 18500 & 249 & 58000 \\
\hline 10 & 60 & 50 & 190 & 90 & 600 & 130 & 1900 & 170 & 6000 & 210 & 19000 & 250 & 60000 \\
\hline 11 & 61.5 & 51 & 195 & 91 & 615 & 131 & 1950 & 171 & 6150 & 211 & 19500 & 251 & 61500 \\
\hline 12 & 63 & 52 & 200 & 92 & 630 & 132 & 2000 & 172 & 6300 & 212 & 20000 & 252 & 63000 \\
\hline 13 & 65 & 53 & 206 & 93 & 650 & 133 & 2060 & 173 & 6500 & 213 & 20600 & 253 & 65000 \\
\hline 14 & 67 & 54 & 212 & 94 & 670 & 134 & 2120 & 174 & 6700 & 214 & 21200 & 254 & 67000 \\
\hline 15 & 69 & 55 & 218 & 95 & 690 & 135 & 2180 & 175 & 6900 & 215 & 21800 & 255 & 69000 \\
\hline 16 & 71 & 56 & 224 & 96 & 710 & 136 & 2240 & 176 & 7100 & 216 & 22400 & 256 & 71000 \\
\hline 17 & 73 & 57 & 230 & 97 & 730 & 137 & 2300 & 177 & 7300 & 217 & 23000 & 257 & 73000 \\
\hline 18 & 75 & 58 & 236 & 98 & 750 & 138 & 2360 & 178 & 7500 & 218 & 23600 & 258 & 75000 \\
\hline 19 & 77.5 & 59 & 243 & 99 & 775 & 139 & 2430 & 179 & 7750 & 219 & 24300 & 259 & 77500 \\
\hline 20 & 80 & 60 & 250 & 100 & 800 & 140 & 2500 & 180 & 8000 & 220 & 25000 & 260 & 80000 \\
\hline 21 & 82.5 & 61 & 257 & 101 & 825 & 141 & 2575 & 181 & 8250 & 221 & 25750 & 261 & 82500 \\
\hline 22 & 85 & 62 & 265 & 102 & 850 & 142 & 2650 & 182 & 8500 & 222 & 26500 & 262 & 85000 \\
\hline 23 & 87.5 & 63 & 272 & 103 & 875 & 143 & 2725 & 183 & 8750 & 223 & 27250 & 263 & 87500 \\
\hline 24 & 90 & 64 & 280 & 104 & 900 & 144 & 2800 & 184 & 9000 & 224 & 2800 & 264 & 90000 \\
\hline 25 & 92.5 & 65 & 290 & 105 & 925 & 145 & 2900 & 185 & 9250 & 225 & 29000 & 265 & 92500 \\
\hline 26 & 95 & 66 & 300 & 106 & 950 & 146 & 3000 & 186 & 9500 & 226 & 30000 & 266 & 95000 \\
\hline 27 & 97.5 & 67 & 307 & 107 & 975 & 147 & 3075 & 187 & 9750 & 227 & 30750 & 267 & 97500 \\
\hline 28 & 100 & 68 & 315 & 108 & 1000 & 148 & 3150 & 188 & 10000 & 228 & 31500 & 268 & 100000 \\
\hline 29 & 103 & 69 & 325 & 109 & 1030 & 149 & 3250 & 189 & 10300 & 229 & 32500 & 269 & 103000 \\
\hline 30 & 106 & 70 & 335 & 110 & 1060 & 150 & 3350 & 190 & 10600 & 230 & 33500 & 270 & 106000 \\
\hline 31 & 109 & 71 & 345 & 111 & 1090 & 151 & 3450 & 191 & 10900 & 231 & 34500 & 271 & 109000 \\
\hline 32 & 112 & 72 & 355 & 112 & 1120 & 152 & 3550 & 192 & 11200 & 232 & 35500 & 272 & 112000 \\
\hline 33 & 115 & 73 & 365 & 113 & 1150 & 153 & 3650 & 193 & 11500 & 233 & 36500 & 273 & 115000 \\
\hline 34 & 118 & 74 & 375 & 114 & 1180 & 154 & 3750 & 194 & 11800 & 234 & 37500 & 274 & 118000 \\
\hline 35 & 121 & 75 & 387 & 115 & 1215 & 155 & 3875 & 195 & 12150 & 235 & 38750 & 275 & 121500 \\
\hline 36 & 125 & 76 & 400 & 116 & 1250 & 156 & 4000 & 196 & 12500 & 236 & 40000 & 276 & 125000 \\
\hline 37 & 128 & 77 & 412 & 117 & 1285 & 157 & 4125 & 197 & 12850 & 237 & 41250 & 277 & 128500 \\
\hline 38 & 132 & 78 & 425 & 118 & 1320 & 158 & 4250 & 198 & 13200 & 238 & 42500 & 278 & 132000 \\
\hline 39 & 136 & 79 & 437 & 119 & 1360 & 159 & 4375 & 199 & 13600 & 239 & 43750 & 279 & 136000 \\
\hline
\end{tabular}


Abbildung 1.2: Tabelle Geschwindigkeits-Index (verändert nach E.T.R.T.O., 2002)

\begin{tabular}{|c|c|}
\hline $\begin{array}{c}\text { Speed Symbol } \\
\text { Code de vitesse } \\
\text { Geschwindigkeits- } \\
\text { Symbol }\end{array}$ & $\begin{array}{c}\text { Speed } \\
\text { Vitesse } \\
\text { Geschwindigkeit } \\
(\mathrm{km} / \mathrm{h})\end{array}$ \\
\hline $\mathrm{A} 1$ & 5 \\
\hline $\mathrm{A} 2$ & 10 \\
\hline A3 & 15 \\
\hline A4 & 20 \\
\hline A5 & 25 \\
\hline A6 & 30 \\
\hline A7 & 35 \\
\hline A8 & 40 \\
\hline $\mathrm{B}$ & 50 \\
\hline $\mathrm{C}$ & 60 \\
\hline $\mathrm{D}$ & 65 \\
\hline$E$ & 70 \\
\hline $\mathrm{F}$ & 80 \\
\hline $\mathrm{G}$ & 90 \\
\hline I & 100 \\
\hline $\mathrm{K}$ & 110 \\
\hline $\mathrm{L}$ & 120 \\
\hline$M$ & 130 \\
\hline $\mathrm{N}$ & 140 \\
\hline $\mathrm{P}$ & 150 \\
\hline$Q$ & 160 \\
\hline $\mathrm{R}$ & 170 \\
\hline $\mathrm{S}$ & 180 \\
\hline$T$ & 190 \\
\hline $\mathrm{U}$ & 200 \\
\hline $\mathrm{H}$ & 210 \\
\hline $\mathrm{V}$ & 240 \\
\hline $\mathrm{W}$ & 270 \\
\hline $\mathrm{Y}$ & 300 \\
\hline
\end{tabular}




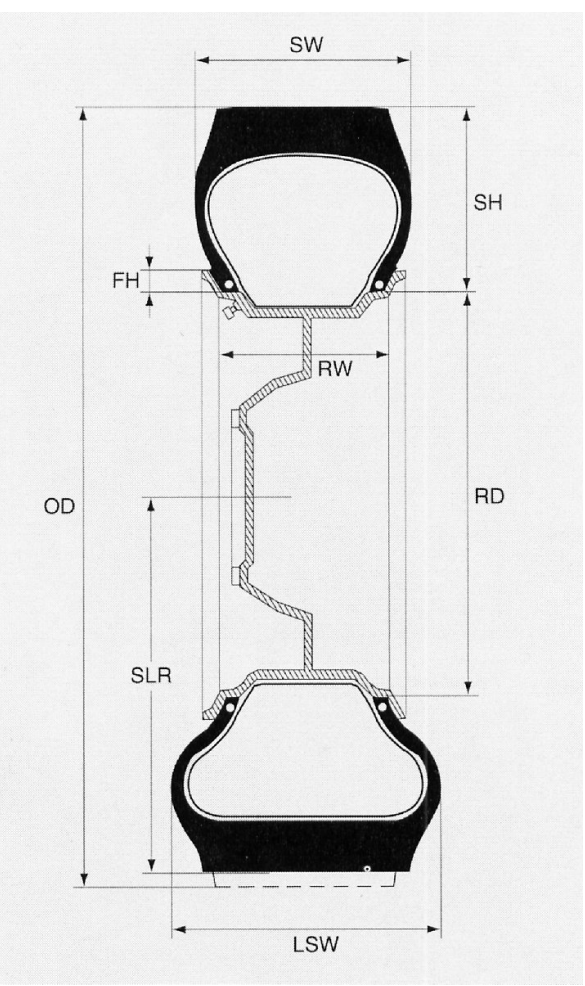

Abbildung 1.3: Reifenmaße im be- und entlasteten Zustand - dargestellt am Reifenquerschnitt (BARUM, 2003)

Die Querschnittsnennbreite (SW) ist ein Konstruktionsmaß, das die Reifenbreite mit glatter Seitenwand angibt.

Die maximale Betriebsbreite ( $L S W$ ) ist die maximal zulässige Breite des Reifen inklusive der Deformation unter Last.

Der Außendurchmesser (OD) bezeichnet den Durchmesser des unbeladenen/unbelasteten Reifens. Er kann mit Hilfe des Umfangs (lo) wie folgt berechnet werden: $D=10 / \pi[\mathrm{mm}]$

Die Querschnittshöhe (SH) ist gleich dem Außendurchmesser abzgl. des Felgendurchmessers geteilt durch zwei.

Der statische Halbmesser (SLR) ist der Abstand zwischen der Achse und der Kontaktfläche eines stehenden Reifens bei vorgeschriebener Last und dem dazugehörigen Fülldruck.

Der Abrollumfang (lo) ist die zurückgelegte Wegstrecke bei einer Radumdrehung eines Reifens auf festem Untergrund.

Felgenmaulweite $(R W)$ ist der Abstand zwischen den gegenüberliegenden Felgenrändern in Zoll.

Felgendurchmesser $(R D)$ ist der Durchmesser der Felge im Bereich der Wulstferse in Zoll.

Felgenhornhöhe $(F H)$ ist der Abstand zwischen der Wulstferse und dem oberen Rand des Felgenhorns. 


\section{Anhang 2}

Tabelle 2.1: In der forstlichen Praxis eingesetzte Diagonalreifen

\begin{tabular}{|c|c|c|}
\hline Kombinationsziffer / Nummerncode & Häufigkeit & Prozent \\
\hline 14,9_378_100_24,0_12_9999_0_3 & 132 &, 5 \\
\hline 14,9_378_100_24,0_14_9999_0_0 & 1378 & 4,7 \\
\hline 14,9_378_100_28,0_12_9999_0_3 & 99 & ,3 \\
\hline 14,9_378_100_28,0_14_9999_0_0 & 245 & ,8 \\
\hline 16,9_429_100_28,0_14_9999_0_0 & 216 & ,7 \\
\hline 16,9_429_100_30,0_14_9999_0_9999 & 1870 & 6,4 \\
\hline 18,4_467_100_30,0_14_9999_0_3 & 30 &, 1 \\
\hline 18,4_467_100_30,0_14_9999_0_9999 & 450 & 1,5 \\
\hline 18,4_467_100_30,0_16_9999_0_0 & 68 & ,2 \\
\hline 18,4_467_100_34,0_14_9999_0_0 & 1578 & 5,4 \\
\hline 18,4_467_100_34,0_14_9999_0_3 & 132 &, 5 \\
\hline 18,4_467_100_38,0_14_9999_0_0 & 245 & 8 \\
\hline 18,4_467_100_38,0_14_9999_0_3 & 99 & ,3 \\
\hline 20,8_528_100_38,0_20_9999_0_0 & 216 & ,7 \\
\hline 23,1_587_100_26,0_14_9999_0_0 & 544 & 1,9 \\
\hline 23,1_587_100_26,0_16_9999_0_0 & 285 & 1,0 \\
\hline 24,5_622_100_32,0_16_9999_0_0 & 104 & ,4 \\
\hline 28L_714_100_26,0_14_9999_0_0 & 492 & 1,7 \\
\hline 28L_714_100_26,0_16_9999_0_0 & 120 & ,4 \\
\hline 9999_400_55_22,5_8_9999_0_1 & 8 & 0 \\
\hline 9999_500_45_22,5_12_9999_0_3 & 1400 & 4,8 \\
\hline 9999_500_60_22,5_16_9999_0_1 & 48 & ,2 \\
\hline 9999_500_60_22,5_16_9999_0_3 & 12 & 0 \\
\hline 9999_500_60_26,5_12_9999_0_0 & 217 & ,7 \\
\hline 9999_500_60_26,5_12_9999_0_3 & 4 & 0 \\
\hline 9999_500_60_26,5_14_9999_0_3 & 79 & ,3 \\
\hline 9999_500_65_26,5_14_9999_0_3 & 76 & ,3 \\
\hline 9999_550_45_22,5_12_9999_0_1 & 168 & ,6 \\
\hline 9999_600_50_22,5_12_9999_0_0 & 136 &, 5 \\
\hline 9999_600_50_22,5_12_9999_0_1 & 216 & ,7 \\
\hline 9999_600_50_22,5_12_9999_0_2 & 1928 & 6,6 \\
\hline 9999_600_50_22,5_16_9999_0_0 & 2579 & 8,8 \\
\hline 9999_600_50_22,5_16_9999_0_1 & 80 & ,3 \\
\hline 9999_600_50_22,5_16_9999_0_2 & 3 & 0 \\
\hline 9999_600_55_22,5_9999_162_0_1 & 96 & ,3 \\
\hline 9999_600_55_26,5_12_9999_0_0 & 74 & ,3 \\
\hline 9999_600_55_26,5_14_9999_0_0 & 10 &, 0 \\
\hline 9999_600_55_26,5_14_9999_0_3 & 1182 & 4,0 \\
\hline 9999_600_55_26,5_16_9999_0_0 & 3117 & 10,6 \\
\hline 9999_600_55_26,5_16_9999_0_1 & 980 & 3,3 \\
\hline 9999_600_55_26,5_16_9999_0_2 & 970 & 3,3 \\
\hline 9999_600_60_30,5_14_9999_0_3 & 1092 & 3,7 \\
\hline 9999_600_65_34,0_14_9999_0_0 & 1164 & 4,0 \\
\hline 9999_600_65_34,0_14_9999_0_2 & 116 & ,4 \\
\hline 9999_600_65_34,0_14_9999_0_3 & 110 & ,4 \\
\hline 9999_650_65_26,5_20_9999_0_1 & 37 & 1 \\
\hline 9999_700_40_22,5_12_9999_0_2 & 8 & 0 \\
\hline 9999_700_40_22,5_16_9999_0_2 & 67 & , 2 \\
\hline 9999_700_45_22,5_16_9999_0_0 & 1565 & 5,3 \\
\hline 9999_700_50_26,5_16_9999_0_0 & 1520 & 5,2 \\
\hline 9999_700_50_26,5_16_9999_0_1 & 624 & 2,1 \\
\hline 9999_700_50_26,5_16_9999_0_2 & 157 &, 5 \\
\hline 9999_700_55_34,0_14_9999_0_0 & 992 & 3,4 \\
\hline 9999_700_55_34,0_14_9999_0_2 & 126 & ,4 \\
\hline 9999_700_70_34,0_16_9999_0_0 & 14 & 0 \\
\hline 9999_800_40_26,5_20_9999_0_2 & 40 & 1 \\
\hline Gesamt & 29318 & 100,0 \\
\hline
\end{tabular}


Tabelle 2.2: In der forstlichen Praxis eingesetzte Radialreifen

\begin{tabular}{|l|r|r|}
\hline Kombinationsziffer/ Nummerncode & Häufigkeit & Prozent \\
\hline 14,9_378_100_24,0_9999_126_1_0 & 830 & 11,3 \\
16,9_429_100_30,0_9999_137_1_0 & 450 & 6,1 \\
18,4_467_100_30,0_9999_142_1_0 & 450 & 6,1 \\
18,4_467_100_34,0_9999_144_1_0 & 830 & 11,3 \\
9999_500_65_24,0_9999_142_1_0 & 130 & 1,8 \\
9999_500_65_28,0_9999_144_1_0 & 1059 & 14,4 \\
9999_500_75_24,0_9999_154_1_0 & 458 & 6,2 \\
9999_520_75_30,0_9999_159_1_0 & 180 & 2,4 \\
9999_540_60_26,5_9999_145_1_0 & 400 & 5,4 \\
9999_540_65_28,0_9999_149_1_0 & 375 & 5,1 \\
9999_540_75_38,0_9999_165_1_0 & 288 & 3,9 \\
9999_600_55_26,5_9999_172_1_1 & 32 &, 4 \\
9999_600_65_34,0_9999_157_1_0 & 16 &, 2 \\
9999_600_65_38,0_9999_159_1_0 & 1501 & 20,4 \\
9999_650_65_38,0_9999_164_1_0 & 367 & 5,0 \\
Gesamt & 7366 & 100,0 \\
\hline
\end{tabular}




\section{Anhang 3}

Tabelle 3.1: Reifendimensionen der Firma Barum

\begin{tabular}{|c|c|c|c|c|c|c|c|c|c|c|c|}
\hline $\begin{array}{l}\text { Reifenher- } \\
\text { steller }\end{array}$ & $\begin{array}{c}\text { Nennbreite } \\
\text { [Zoll] }\end{array}$ & $\begin{array}{l}\text { Nennbreite } \\
\text { [mm] }\end{array}$ & $\begin{array}{c}\text { H/B-Verhältnis } \\
\text { [\%] }\end{array}$ & $\begin{array}{l}\text { Felgendurch- } \\
\text { messer } \\
\text { [Zoll] }\end{array}$ & $\begin{array}{l}\text { Load-/Speed- } \\
\text { Index (LI/SI) }\end{array}$ & PR-Zahl & Bauweise & $\begin{array}{l}\text { Profiltiefe } \\
\text { [mm] }\end{array}$ & Profil & $\begin{array}{l}\text { Durchmesser } \\
\text { [mm] }\end{array}$ & \begin{tabular}{|c|} 
Laufende \\
Nr.
\end{tabular} \\
\hline Barum & 16.9 & 429 & - & 30 & - & 12 & diagonal & 32 & TL 1 & 1420 & 01 \\
\hline
\end{tabular}

Tabelle 3.2: Reifendimensionen der Firma Firestone

\begin{tabular}{|c|c|c|c|c|c|c|c|c|c|c|c|}
\hline $\begin{array}{l}\text { Reifenher- } \\
\text { steller }\end{array}$ & $\begin{array}{c}\text { Nennbreite } \\
\text { [Zoll] }\end{array}$ & $\begin{array}{l}\text { Nennbreite } \\
{[\mathrm{mm}]}\end{array}$ & $\begin{array}{c}\text { H/B-Verhältnis } \\
\text { [\%] }\end{array}$ & $\begin{array}{l}\text { Felgendurch- } \\
\text { messer } \\
\text { [Zoll] }\end{array}$ & $\begin{array}{l}\text { Load-/Speed- } \\
\text { Index (LI/SI) }\end{array}$ & PR-Zahl & Bauweise & $\begin{array}{l}\text { Profiltiefe } \\
\text { [mm] }\end{array}$ & Profil & $\begin{array}{l}\text { Durchmesser } \\
{[\mathrm{mm}]}\end{array}$ & $\begin{array}{c}\text { Laufende } \\
\text { Nr. }\end{array}$ \\
\hline Firestone & 23.1 & 587 & - & 26 & - & 14 & diagonal & 53 & $\begin{array}{l}\text { Forestry } \\
\text { Special }\end{array}$ & 1613 & 01 \\
\hline Firestone & $28 \mathrm{~L}$ & 714 & - & 26 & - & 14 & diagonal & 56 & $\begin{array}{l}\text { Forestry } \\
\text { Special }\end{array}$ & 1638 & 02 \\
\hline
\end{tabular}

Tabelle 3.3: Reifendimensionen der Firma Michelin

\begin{tabular}{|c|c|c|c|c|c|c|c|c|c|c|c|}
\hline $\begin{array}{l}\text { Reifenher- } \\
\text { steller }\end{array}$ & $\begin{array}{c}\text { Nennbreite } \\
\text { [Zoll] }\end{array}$ & $\begin{array}{l}\text { Nennbreite } \\
{[\mathrm{mm}]}\end{array}$ & $\begin{array}{c}\text { H/B- } \\
\text { Verhältnis } \\
{[\%]} \\
\%]\end{array}$ & $\begin{array}{c}\text { Felgendurch- } \\
\text { messer } \\
\text { [Zoll] }\end{array}$ & $\begin{array}{l}\text { Load-/Speed- } \\
\text { Index (LI/SI) }\end{array}$ & PR-Zahl & Bauweise & $\begin{array}{l}\text { Profiltiefe } \\
\text { [mm] }\end{array}$ & Profil & $\begin{array}{l}\text { Durchmesser } \\
{[\mathrm{mm}]}\end{array}$ & $\begin{array}{c}\text { Laufende } \\
\text { Nr. }\end{array}$ \\
\hline Michelin & 14.9 & 378 & - & 24 & 126/A8 & - & radial & 41 & XM27 & 1252 & 01 \\
\hline Michelin & 18.4 & 467 & - & 34 & 144/A8 & - & radial & 42 & XM27 & 1550 & 02 \\
\hline Michelin & - & 500 & 75 & 24 & 154/A8 & - & radial & 44 & FOREXBIB & 1345 & 03 \\
\hline Michelin & - & 520 & 75 & 26 & 157/A8 & - & radial & 48 & FOREXBIB & 1482 & 04 \\
\hline Michelin & - & 520 & 75 & 30 & 159/A8 & - & radial & 47 & FOREXBIB & 1566 & 05 \\
\hline Michelin & - & 540 & 75 & 38 & $165 /$ A8 & - & radial & 48 & FOREXBIB & 1799 & 06 \\
\hline Michelin & - & 620 & 75 & 26 & 167/A8 & - & radial & 46 & FOREXBIB & 1621 & 07 \\
\hline
\end{tabular}

\section{Bemessung auf weichem Untergrund}

Bemessung auf hartem Untergrund

Bemessung auf weichem/hartem Untergrund 
Tabelle 3.4: Reifendimensionen der Firma Nokian

\begin{tabular}{|c|c|c|c|c|c|c|c|c|c|c|c|}
\hline $\begin{array}{l}\text { Reifenher- } \\
\text { steller }\end{array}$ & $\begin{array}{c}\text { Nennbreite } \\
\text { [Zoll] }\end{array}$ & $\begin{array}{c}\text { Nennbreite } \\
{[\mathrm{mm}]}\end{array}$ & $\begin{array}{c}\text { H/B-Verhältnis } \\
{[\%]}\end{array}$ & $\begin{array}{c}\text { Felgendurch- } \\
\text { messer } \\
\text { [Zoll] }\end{array}$ & $\begin{array}{l}\text { Load-/Speed- } \\
\text { Index (LI/SI) }\end{array}$ & PR-Zahl & Bauweise & $\begin{array}{l}\text { Profiltiefe } \\
{[\mathrm{mm}]}\end{array}$ & Profil & $\begin{array}{l}\text { Durchmesser } \\
{[\mathrm{mm}]}\end{array}$ & $\begin{array}{c}\text { Laufende } \\
\text { Nr. }\end{array}$ \\
\hline Nokian & 14.9 & 378 & - & 24 & 138/A8 & 14 & diagonal & 44 & FS DT & 1265 & 33 \\
\hline Nokian & 18.4 & 467 & - & 34 & 154/A8 & 14 & diagonal & 48 & FS DT & 1650 & 17 \\
\hline Nokian & 18.4 & 467 & - & 38 & $155 /$ A8 & 14 & diagonal & 53 & TR Forest & 1750 & 12 \\
\hline Nokian & 23.1 & 587 & - & 26 & - & 16 & diagonal & 54 & TRS LS-2 & 1632 & 05 \\
\hline Nokian & $28 \mathrm{~L}$ & 714 & - & 26 & - & 16 & diagonal & 57 & TRS LS2 & 1644 & 06 \\
\hline Nokian & - & 500 & 65 & 24 & 142/A8 & - & radial & 48 & TR Forest & 1234 & 13 \\
\hline Nokian & - & 500 & 60 & 26.5 & - & 12 & diagonal & 53 & TRS LS-2 & 1271 & 11 \\
\hline Nokian & - & 500 & 65 & 28 & $144 / \mathrm{A} 8$ & - & radial & 48 & TR Forest & 1345 & 27 \\
\hline Nokian & - & 540 & 60 & 26.5 & $145 / \mathrm{A} 8$ & - & radial & 48 & TR Forest & 1321 & 32 \\
\hline Nokian & - & 540 & 65 & 28 & 149/A8 & - & radial & 51 & TR Forest & 1413 & 07 \\
\hline Nokian & - & 550 & 45 & 22.5 & - & 12 & diagonal & 20 & ELS & 1067 & 04 \\
\hline Nokian & - & 600 & 50 & 22.5 & - & 16 & diagonal & 42 & TRS LS-2 & 1170 & 23 \\
\hline Nokian & - & 600 & 50 & 22.5 & - & 12 & diagonal & 42 & TRS LS-2 & 1170 & 22 \\
\hline Nokian & - & 600 & 50 & 22.5 & - & 16 & diagonal & 25 & ELS L-2 & 1170 & 21 \\
\hline Nokian & - & 600 & 55 & 26.5 & - & 12 & diagonal & 30 & ELS & 1333 & 02 \\
\hline Nokian & - & 600 & 55 & 26.5 & - & 12 & diagonal & 53 & TRS LS-2 & 1333 & 01 \\
\hline Nokian & - & 600 & 55 & 26.5 & - & 16 & diagonal & 38 & ELS L-2 & 1333 & 30 \\
\hline Nokian & - & 600 & 55 & 26.5 & - & 16 & diagonal & 53 & TRS LS-2 & 1333 & 29 \\
\hline Nokian & - & 600 & 55 & 26.5 & - & 20 & diagonal & 38 & ELS L-2 & 1333 & 24 \\
\hline Nokian & - & 600 & 65 & 34 & 157/A8 & - & radial & 52 & TR Forest & 1664 & 14 \\
\hline Nokian & - & 600 & 65 & 34 & - & 14 & diagonal & 51 & TRS L-2 & 1644 & 10 \\
\hline Nokian & - & 600 & 65 & 38 & 159/A8 & - & radial & 52 & TR Forest & 1745 & 28 \\
\hline Nokian & - & 650 & 65 & 26.5 & - & 20 & diagonal & 35 & ELS L-2 & 1485 & 31 \\
\hline Nokian & - & 650 & 65 & 26.5 & - & 12 & diagonal & 35 & ELS L-2 & 1480 & 19 \\
\hline Nokian & - & 650 & 65 & 38 & 164/A8 & - & radial & 53 & TR Forest & 1810 & 08 \\
\hline Nokian & - & 700 & 45 & 22.5 & - & 16 & diagonal & 42 & TRS LS-2 & 1150 & 25 \\
\hline Nokian & - & 700 & 50 & 26.5 & - & 20 & diagonal & 53 & TRS LS-2 & 1333 & 26 \\
\hline Nokian & - & 700 & 50 & 26.5 & - & 16 & diagonal & 38 & ELS L-2 & 1350 & 20 \\
\hline Nokian & - & 700 & 55 & 34 & - & 14 & diagonal & 51 & TRS LS-2 & 1630 & 18 \\
\hline Nokian & - & 700 & 50 & 26.5 & - & 16 & diagonal & 53 & TRS LS-2 & 1333 & 03 \\
\hline Nokian & - & 750 & 55 & 26.5 & 177/A8 & - & diagonal & 50 & TRS L-2 & 1485 & 15 \\
\hline Nokian & - & 800 & 40 & 26.5 & 170/A8 & - & diagonal & 30 & Forest King F & 1340 & 16 \\
\hline
\end{tabular}

Bemessung auf weichem Untergrund

Bemessung auf weichem/hartem Untergrund 
Tabelle 3.5: Reifendimensionen der Firma Trelleborg

\begin{tabular}{|c|c|c|c|c|c|c|c|c|c|c|c|}
\hline $\begin{array}{l}\text { Reifenher- } \\
\text { steller }\end{array}$ & $\begin{array}{c}\text { Nennbreite } \\
\text { [Zoll] }\end{array}$ & $\begin{array}{l}\text { Nennbreite } \\
\text { [mm] }\end{array}$ & $\begin{array}{c}\text { H/B-Verhältnis } \\
{[\%]}\end{array}$ & $\begin{array}{c}\text { Felgendurch- } \\
\text { messer } \\
\text { [Zoll] }\end{array}$ & $\begin{array}{l}\text { Load-/Speed- } \\
\text { Index (LI/SI) }\end{array}$ & PR-Zahl & Bauweise & $\begin{array}{l}\text { Profiltiefe } \\
\text { [mm] }\end{array}$ & Profil & $\begin{array}{l}\text { Durchmesser } \\
{[\mathrm{mm}]}\end{array}$ & $\begin{array}{c}\text { Laufende } \\
\text { Nr. }\end{array}$ \\
\hline Trelleborg & 14.9 & 378 & - & 24 & - & 12 & diagonal & 37 & R1 & 1165 & 01 \\
\hline Trelleborg & 14.9 & 378 & - & 28 & - & 12 & diagonal & 40 & 406 & 1365 & 02 \\
\hline Trelleborg & 18.4 & 467 & - & 34 & - & 14 & diagonal & 55 & 410 & 1650 & 03 \\
\hline Trelleborg & 18.4 & 467 & - & 38 & - & 14 & diagonal & 45 & 407 & 1750 & 04 \\
\hline Trelleborg & - & 500 & 45 & 22.5 & - & 12 & diagonal & 20 & 404 & 1020 & 05 \\
\hline Trelleborg & - & 500 & 60 & 22.5 & - & 16 & diagonal & 20 & 404 & 1170 & 21 \\
\hline Trelleborg & - & 500 & 60 & 26.5 & - & 14 & diagonal & 50 & 416 & 1250 & 10 \\
\hline Trelleborg & - & 500 & 65 & 26.5 & - & 14 & diagonal & 50 & 425 & 1325 & 11 \\
\hline Trelleborg & - & 600 & 50 & 22.5 & 143/A8 & - & diagonal & 35 & 428 & 1170 & 07 \\
\hline Trelleborg & - & 600 & 50 & 22.5 & - & 12 & diagonal & 35 & 423 & 1200 & 25 \\
\hline Trelleborg & - & 600 & 55 & 26.5 & 154/A8 & - & diagonal & 40 & 428 & 1350 & 20 \\
\hline Trelleborg & - & 600 & 55 & 26.5 & - & 14 & diagonal & 50 & 414 & 1350 & 26 \\
\hline Trelleborg & - & 600 & 55 & 26.5 & 148/A8 & - & diagonal & 45 & 422 & 1350 & 29 \\
\hline Trelleborg & - & 600 & 55 & 26.5 & - & 16 & diagonal & 40 & 421 & 1350 & 30 \\
\hline Trelleborg & - & 600 & 60 & 30.5 & - & 14 & diagonal & 50 & 414 & 1495 & 12 \\
\hline Trelleborg & - & 600 & 60 & 30.5 & - & 20 & diagonal & 50 & 421 & 1495 & 13 \\
\hline Trelleborg & - & 600 & 65 & 34 & - & 14 & diagonal & 50 & 422 & 1650 & 16 \\
\hline Trelleborg & - & 600 & 65 & 34 & - & 14 & diagonal & 50 & 414 & 1650 & 17 \\
\hline Trelleborg & - & 620 & 55 & 30.5 & 152/A8 & - & diagonal & 40 & 428 & 1465 & 14 \\
\hline Trelleborg & - & 620 & 55 & 30.5 & 158/A8 & - & diagonal & 40 & 428 & 1465 & 15 \\
\hline Trelleborg & - & 650 & 45 & 22.5 & 144/A8 & - & diagonal & 45 & 422 & 1160 & 08 \\
\hline Trelleborg & - & 650 & 45 & 22.5 & 135/A8 & - & diagonal & 45 & 422 & 1160 & 09 \\
\hline Trelleborg & - & 700 & 40 & 22.5 & - & 16 & diagonal & 35 & 423 & 1170 & 06 \\
\hline Trelleborg & - & 700 & 50 & 26.5 & - & 16 & diagonal & 40 & 423 & 1350 & 23 \\
\hline Trelleborg & - & 710 & 40 & 22.5 & 151/A8 & - & diagonal & 35 & 428 & 1170 & 18 \\
\hline Trelleborg & - & 710 & 45 & 26.5 & 151/A8 & - & diagonal & 45 & 422 & 1357 & 24 \\
\hline Trelleborg & - & 710 & 45 & 26.5 & 151/A8 & - & diagonal & 40 & 428 & 1357 & 27 \\
\hline Trelleborg & - & 710 & 55 & 34 & 161/A8 & - & diagonal & 51 & 422 & 1650 & 19 \\
\hline Trelleborg & - & 750 & 45 & 30.5 & 169/A8 & - & diagonal & 40 & 428 & 1465 & 22 \\
\hline
\end{tabular}

Bemessung auf weichem Untergrund

Bemessung auf weichem/hartem Untergrund 
Tabelle 3.6: Reifendimensionen der Firmen Nokian und Trelleborg, die mit Wasser befüllt wurden

\begin{tabular}{|c|c|c|c|c|c|c|c|c|c|c|c|}
\hline $\begin{array}{l}\text { Reifenher- } \\
\text { steller }\end{array}$ & $\begin{array}{c}\text { Nennbreite } \\
\text { [Zoll] }\end{array}$ & $\begin{array}{l}\text { Nennbreite } \\
\text { [mm] }\end{array}$ & $\begin{array}{c}\text { H/B-Verhältnis } \\
{[\%]}\end{array}$ & $\begin{array}{l}\text { Felgendurch- } \\
\text { messer } \\
\text { [Zoll] }\end{array}$ & $\begin{array}{l}\text { Load-/Speed- } \\
\text { Index (LI/SI) }\end{array}$ & PR-Zahl & Bauweise & $\begin{array}{l}\text { Profiltiefe } \\
{[\mathrm{mm}]}\end{array}$ & Profil & $\begin{array}{c}\text { Durchmesser } \\
{[\mathrm{mm}]}\end{array}$ & $\begin{array}{c}\text { Laufende } \\
\text { Nr. }\end{array}$ \\
\hline Nokian & - & 700 & 50 & 26.5 & - & 16 & diagonal & 53 & TRS LS-2 & 1333 & 03 \\
\hline Nokian & - & 700 & 50 & 26.5 & - & 16 & diagonal & 38 & ELS L-2 & 1350 & 20 \\
\hline Nokian & - & 600 & 55 & 26.5 & - & 16 & diagonal & 38 & ELS L-2 & 1333 & 30 \\
\hline Nokian & - & 540 & 60 & 26.5 & 145/A8 & - & radial & 48 & TR Forest & 1321 & 32 \\
\hline Trelleborg & - & 600 & 50 & 22.5 & 143/A8 & - & diagonal & 35 & 428 & 1170 & 07 \\
\hline Trelleborg & - & 700 & 50 & 26.5 & - & 16 & diagonal & 40 & 423 & 1350 & 23 \\
\hline Trelleborg & - & 600 & 50 & 22.5 & - & 12 & diagonal & 35 & 423 & 1200 & 25 \\
\hline Trelleborg & - & 710 & 45 & 26.5 & $151 / \mathrm{A} 8$ & - & diagonal & 40 & 428 & 1357 & 27 \\
\hline
\end{tabular}

\section{Bemessung auf weichem/hartem Untergrund mit Wasserfüllung}




\section{Anhang 4}
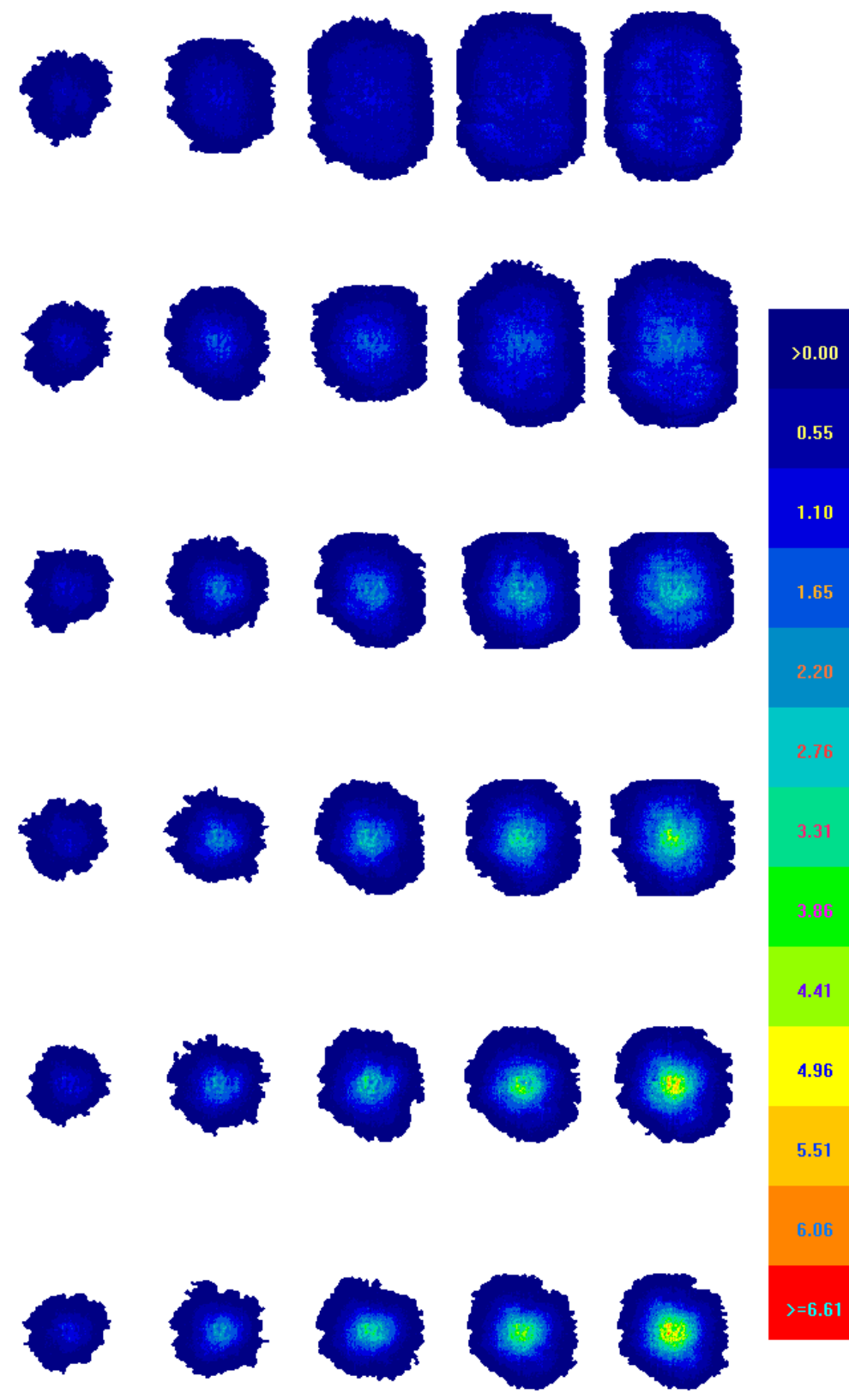

$>=6.61$
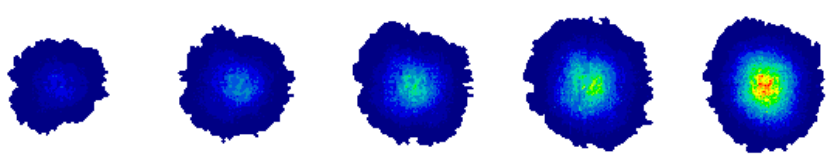

Abbildung 4.1: Darstellung der Kontaktflächenabdrucke des Reifens Trelleborg 600/55-26.5 428 (T20) nach Last- und Fülldruckstufen. Horizontal sind die fünf Laststufen $(10 \mathrm{kN}, 20 \mathrm{kN}, 30 \mathrm{kN}, 40 \mathrm{kN}, 50 \mathrm{kN})$ und vertikal die sieben Fülldruckstufen (0,5 bar, 1,0 bar, 1,5 bar, 2,0 bar, 3,0 bar, 4,0 bar, 5,0 bar) abgetragen. Anhand der unterschiedlichen Farbabstufungen ist die Verteilung der Druckbelastung zu erkennen (der Druck nimmt ab über die Farben ROT / ORANGE / GELB / GRÜN / BLAU). 


\section{Reifenhersteller: Trelleborg} Laufende Nummer: T20

$\begin{array}{ll}\text { Nennbreite [mm]: } & 600 \\ \text { Höhen-Breiten-Verhältnis [\%]: } & 55 \\ \text { Felgendurchmesser [Zoll]: } & 26.5 \\ \text { Load-/Speedindex: } & 154 / \text { A8 } \\ \text { Bauweise: } & \text { diagonal } \\ \text { Profil: } & 428 \\ \text { Durchmesser [mm]: } & 1350\end{array}$

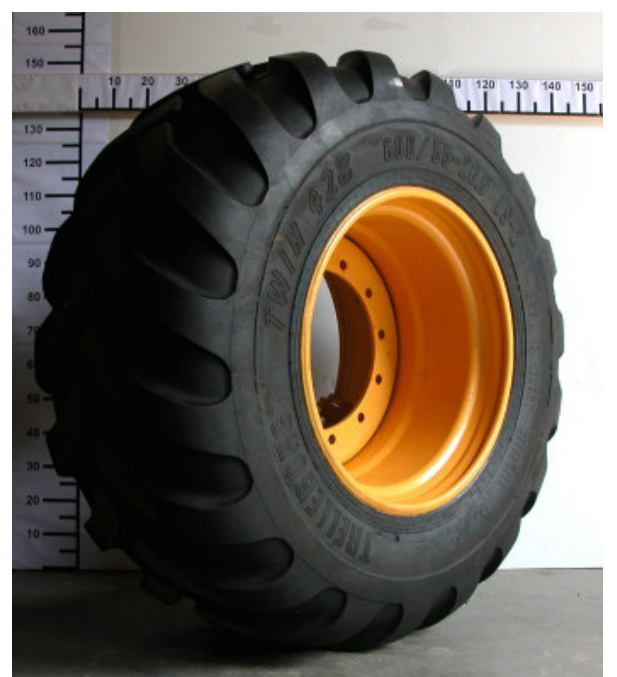

Abbildung 4.2: Schrägansicht des Reifens Trelleborg 600/55-26.5 428 (T20)

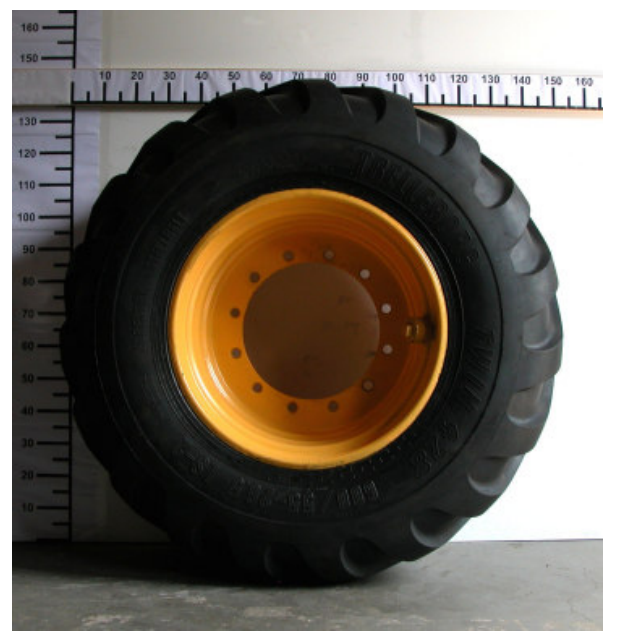

Abbildung 4.3: Seitenansicht des Reifens Trelleborg 600/55-26.5 428 (T20) 


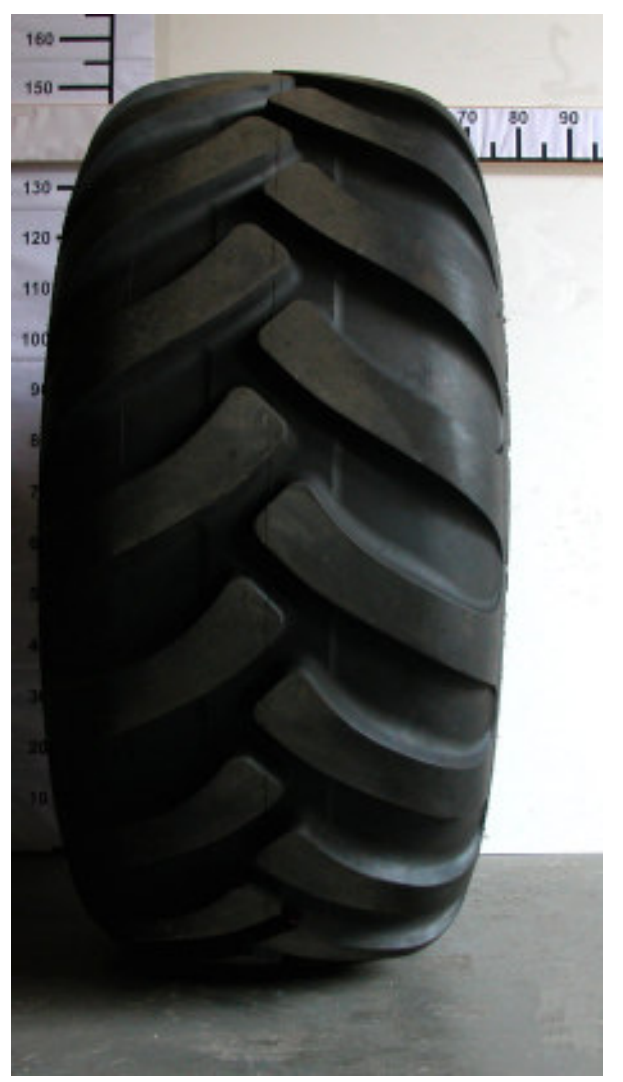

Abbildung 4.4: Frontalansicht des Reifens Trelleborg 600/55-26.5 428 (T20)

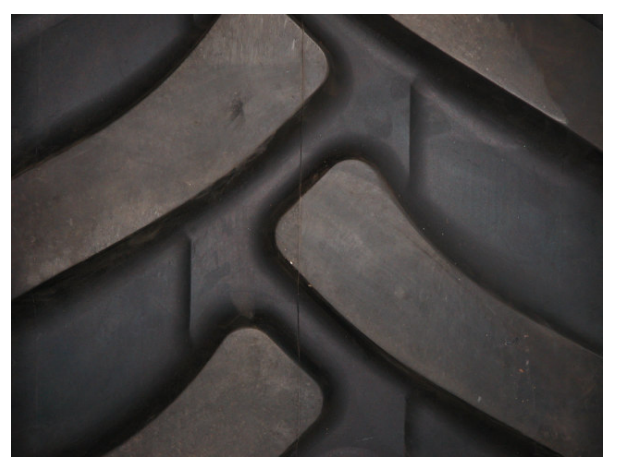

Abbildung 4.5: Profilansicht des Reifens Trelleborg 600/55-26.5 428 (T20) 


\section{Anhang 5}

Tabelle 5.1:Referenzreifen - Berechnungsgrößen für den Formelvergleich

\begin{tabular}{|c|l|l|l|l|l|l|}
\hline Reifennummer & $\begin{array}{c}\text { Reifeninnendruck } \\
{[\mathrm{bar}]}\end{array}$ & $\begin{array}{c}\text { Radllast } \\
{[\mathrm{kN}]}\end{array}$ & $\begin{array}{c}\text { Nennbreite } \\
{[\mathrm{m}]}\end{array}$ & $\begin{array}{c}\text { Durchmesser } \\
{[\mathrm{m}]}\end{array}$ & $\begin{array}{c}\text { Einfederung } \\
{[\mathrm{m}]}\end{array}$ & $\begin{array}{c}\text { Refenhöhe } \\
{[\mathrm{m}]}\end{array}$ \\
\hline N07 & 2,5 & 25 & 0,540 & 1,413 & 0,062 & 0,32 \\
\hline N30 & 2,5 & 25 & 0,6 & 1,333 & 0,047 & 0,31 \\
\hline T23 & 2,5 & 25 & 0,7 & 1,350 & 0,046 & 0,33 \\
\hline T12 & 2,5 & 25 & 0,6 & 1,650 & 0,057 & 0,38 \\
\hline
\end{tabular}




\section{Lebenslauf}

\section{Persönliche Daten}

Name

Geburtsdatum

Geburtsort

Familienstand

\section{Ausbildung und Studium}

\section{- Berufsausbildung}

10/1987 - 03/1990

- Zweiter Bildungsweg

08/1991 - 06/1994

- Studium

10/1994 - 03/1995

04/1995 - 11/1999
Andreas Ebel

21. März 1971

Celle

ledig
Ausbildung zum Polizeivollzugsbeamten im Bundesgrenzschutz

Abendgymnasium Braunschweig, Abitur

Studienbegleitendes Praktikum im Staatlichen Forstamt Lüß in Unterlüß, Landesforstverwaltung Niedersachsen Student der Forstwissenschaften an der Georg-AugustUniversität Göttingen

Studienabschluss: Diplom-Forstwirt

- Praktika während des Studiums

07/1996 - 08/1996

$09 / 1997$

\section{Berufserfahrung}

04/1990 - 09/1994

$12 / 1999-07 / 2001$

\section{Wissenschaftliche Tätigkeit}

08/2001 - 08/2005
Fünfwöchiges Praktikum in der Bodenverwertungsund -verwaltungs GmbH (BVVG), Niederlassung Schwerin Vierwöchiges Praktikum in einem holzverarbeitenden Unternehmen in Schottland, Adam Wilson \& Sons Ltd., Troon

Verwendung in der Grenzschutzabteilung West I in Gifhorn, Dienst in einer Spezialeinheit Manager Marketing/Sales, newtron AG (InternetUnternehmen), Frankfurt am Main, Beratung von Unternehmen des Anlagen- und Maschinenbaus

Wissenschaftlicher Mitarbeiter am Institut für Forstliche Arbeitswissenschaft und Verfahrenstechnologie der Georg-August-Universität Göttingen 